\title{
THE BEAM DYNAMICS AND BEAM RELATED UNCERTAINTIES IN FERMILAB MUON $g-2$ EXPERIMENT
}

\author{
A Dissertation \\ presented in partial fulfillment of the requirements \\ for the degree of Doctor of Philosophy \\ in the Department of Physics and Astronomy
}

The University of Mississippi

by

WANWEI WU

May 2018 
Copyright @ 2018 by Wanwei Wu ALL RIGHTS RESERVED. 


\begin{abstract}
The anomaly of the muon magnetic moment, $a_{\mu} \equiv(g-2) / 2$, has played an important role in constraining physics beyond the Standard Model for many years. Currently, the Standard Model prediction for $a_{\mu}$ is accurate to 0.42 parts per million (ppm). The most recent muon $g-2$ experiment was done at Brookhaven National Laboratory (BNL) and determined $a_{\mu}$ to $0.54 \mathrm{ppm}$, with a central value that differs from the Standard Model prediction by 3.3-3.6 standard deviations and provides a strong hint of new physics. The Fermilab Muon $g-2$ Experiment has a goal to measure $a_{\mu}$ to unprecedented precision: $0.14 \mathrm{ppm}$, which could provide an unambiguous answer to the question whether there are new particles and forces that exist in nature. To achieve this goal, several items have been identified to lower the systematic uncertainties. In this work, we focus on the beam dynamics and beam associated uncertainties, which are important and must be better understood. We will discuss the electrostatic quadrupole system, particularly the hardware-related quad plate alignment and the quad extension and readout system. We will review the beam dynamics in the muon storage ring, present discussions on the beam related systematic errors, simulate the 3D electric fields of the electrostatic quadrupoles and examine the beam resonances. We will use a fast rotation analysis to study the muon radial momentum distribution, which provides the key input for evaluating the electric field correction to the measured $a_{\mu}$.
\end{abstract}




\section{DEDICATION}

To my father and mother, for your love, support and cultivation.

Also to my girlfriend, Shen, for your love, understanding and encouragement. 


\section{ACKNOWLEDGEMENTS}

I would like to express my deepest appreciation to my advisor Breese Quinn for his patience, forbearance and continuous support. Without his assistance and guidance, this thesis would not have been possible. His valuable advice and comments have helped me a lot during my graduate study. I would like to thank the rest of my thesis committee members: Alakabha Datta, Donald Summers and Nathan Hammer for their insightful comments and precious time. I am extremely grateful to Alakabha Datta for his guidance in theoretical high energy physics and to Donald Summers for his instruction of accelerator physics. I also want to thank all the faculty, staff and graduate students at Ole Miss Department of Physics and Astronomy. A special thanks to Luca Bombelli, Kevin Beach, Emanuele Berti, Lucien Cremaldi, Josh Gladden, David Sanders, Ginger Dykes, Amy Barnes for their kind help all the time. Thanks to Hongkai Liu, Liang Chen, Mir Emad Aghili, Saeed Kamali, Khagendra Adhikari, Hector Okada da Silva and Meghna Bhattacharya for studying together.

The work in this thesis would not be possible without the collective contributions of the entire Fermilab Muon g-2 Collaboration. In particular, I would like to thank Hogan Nguyen, Bill Morse, Chris Polly, Erik Ramberg, Rob Carey, Vladimir Tishchenko, Diktys Stratakis, Yannis Semertzidis, David Hertzog, Brendan Casey, Brendan Kiburg, Lee Roberts, Adam Lyon, David Rubin, Peter Winter, Jarek Kaspar, Chris Stoughton, Erik Swanson and Michael Syphers, for their eagerness and unyielding motivation to do a better experiment, and for their invaluable assistance and thoughtful suggestions with my research life. I would also

like to thank Jason D. Crnkovic, Jenny Holzbauer, Kim Siang Khaw, Joe Grange, Sudeshna Ganguly, Yaqian Wang, James Stapleton, Ran Hong, Tammy Walton, Wes Gohn, Karie Badgley, Alex Herrod, Nick Kinnaird, Cristina Schlesier, Robin Bjorkquist, Aaron Fienberg, and Nathan Froemming for their useful comments and helpful discussions, without which 
I would have been lonely. Their inspiration, encouragement and companionship are truly meaningful to me.

In addition, I would like to thank my parents. As rustic and kind-hearted farmers, they try to understand and support me all the way. A special thanks to my girlfriend Shen Liu for her encouragement, understanding, patience, love and support. I also want to thank all of my other family and friends for their support along the way.

I would like to thank Fermi National Accelerator Laboratory (Fermilab), a U. S. Department of Energy, Office of Science, HEP User Facility for the resources provided. Fermilab is managed by Fermi Research Alliance, LLC (FRA), acting under Contract No. DE-AC0207CH11359. The work described in this thesis was supported by the U.S. Department of Energy Office of Science, Office of High Energy Physics, award DE-SC0012391 and a Universities Research Association Visiting Scholar award. 


\section{CONTENTS}

ABSTRACT ii

$\begin{array}{ll}\text { DEDICATION } & \text { iii }\end{array}$

ACKNOWLEDGEMENTS IV iv

LIST OF TABLES I ix

LIST OF FIGURES - X

1 INTRODUCTION 1

1.1 The Standard Model of Particle Physics . . . . . . . . . . . . . . . . . . . 1

1.2 Anomalous Magnetic Moment . . . . . . . . . . . . . . . . . . . . . 3

1.3 Muon g-2: Standard Model and New Physics . . . . . . . . . . . . . . . 3

1.4 Muon $g-2$ Experiments in History . . . . . . . . . . . . . . . . . . . . 11

2 THE FERMILAB MUON $g-2$ EXPERIMENT (E989) 20

2.1 Overview . . . . . . . . . . . . . . . . . . . . 20

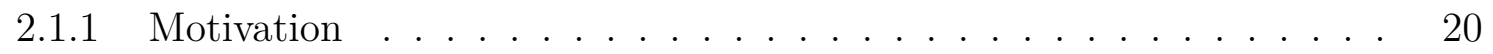

2.1.2 Project Scope . . . . . . . . . . . . . . . . . 21

2.2 Principle . . . . . . . . . . . . . . . . . . . . 22

2.2.1 Measurement of $\vec{\omega}_{a} \ldots \ldots \ldots$. . . . . . . . . . . . 24

2.2.2 Measurement of the Magnetic Field $\vec{B}$. . . . . . . . . . . . . . . 27

2.2.3 Extraction of Muon Anomaly $a_{\mu} \ldots \ldots . . . . . . . . . .27$

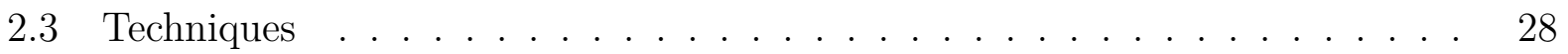

2.3.1 Production of Polarized Muons . . . . . . . . . . . . . . . . . . 28

2.3.2 Storage of Muons . . . . . . . . . . . . . . . . . . . . . . . . . . 30

2.3.3 Monitoring the Muon Beam . . . . . . . . . . . . . . 35

2.3.4 Detecting the Decay Positron Signals . . . . . . . . . . . . . 38

2.4 Statistical and Systematic Errors _. . . . . . . . . . . . . . 38

2.4.1 Statistical Errors . . . . . . . . . . . . . . . . . . . . . 39

$2.4 .2 \omega_{a}$ Systematic Errors . . . . . . . . . . . . . . . . . . 40 
$2.4 .3 \omega_{p}$ Systematic Errors . . . . . . . . . . . . . . . . . . . . 42

$3 \quad$ ELECTROSTATIC QUADRUPOLE SYSTEM 43

3.1 Quad Plate Alignment . . . . . . . . . . . . . . . . . . . . . 43

3.1.1 Alignment Requirements . . . . . . . . . . . . . . . . . . . 45

3.1.2 Investigation of Quad Plate Alignment with Capacitec . . . . . . . 46

3.1.3 Alignment with Micrometer Tools . . . . . . . . . . . . . . . . . . 53

3.1.4 Laser Alignment System . . . . . . . . . . . . . . . . . . . . 58

3.1 .5 Q1 Outer Plate Alignment . . . . . . . . . . . . . . . . . . 59

3.1.6 Final Results of Quad Plate Alignment . . . . . . . . . . . . . 62

3.2 Quad Extension and Readout Systems . . . . . . . . . . . . . . . . 62

3.2.1 Installation of Quad Extension . . . . . . . . . . . . . . . 62

3.2.2 Potting Resistors . . . . . . . . . . . . . . . . . . . 64

3.2 .3 Quad Readout . . . . . . . . . . . . . . . . . . . . . . 64

4 BEAM DYNAMICS IN THE MUON STORAGE RING 69

4.1 Equation of Motion . . . . . . . . . . . . . . . . . . . 69

4.2 Beam Related Systematic Errors _ . . . . . . . . . . . . . . . . 72

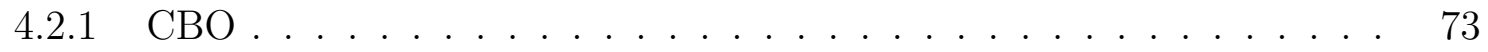

$4.2 .2 \quad$ Lost Muons . . . . . . . . . . . . . . . . . . . . . . . . . . 75

$4.2 .3 \quad$ E-field and Pitch Corrections . . . . . . . . . . . . 76

4.3 3D E-field from OPERA-3D . . . . . . . . . . . . . 76

4.3.1 Geometry for OPERA-3D Simulation . . . . . . . . . . . . 78

4.3.2 The 3D Electric Field Map _ . . . . . . . . . . . . . . . . . . . 79

4.4 Beam Resonances . . . . . . . . . . . . . . . . . . . . . . . . . 81

4.4 .1 Principles . . . . . . . . . . . . . . . . . . . . . . . . 82

$4.4 .2 \quad \mathrm{~g}-2$ Beam Resonances . . . . . . . . . . . . . . . . . . 83

$5 \quad$ FAST ROTATION ANALYSIS $\quad 87$

5.1 Motivation and Ideas . . . . . . . . . . . . . . . . . . . . . . 87

5.2 Fourier Transform Method . . . . . . . . . . . . . . . . . . . . . 87

$5.3 \chi^{2}$ Minimization Method . . . . . . . . . . . . . . . . . 88

5.3 .1 FRA Assumptions . . . . . . . . . . . . . . . . . . 90

5.3 .2 Mathematical Details . . . . . . . . . . . . . . . . . 91

5.3 .3 Calculation of $\beta_{i j} \ldots \ldots \ldots \ldots \ldots \ldots$

5.3.4 Solve the Radial Bin Content $f_{i}$ by Matrix . . . . . . . . . . . . . . 94

5.3 .5 Simulation . . . . . . . . . . . . . . . . . . . 95

5.3 .6 Commissioning Data . . . . . . . . . . . . . . . . . . . . . 100 
5.4 Electric Field Correction . . . . . . . . . . . . . . . . . . . . 110

6 CONCLUSION 112

6.1 Electrostatic Quadrupole System . . . . . . . . . . . . . . . . . 112

6.2 Beam Dynamics in Muon Storage Ring . . . . . . . . . . . . . . . . . . . . 113

6.3 Fast Rotation Analysis . . . . . . . . . . . . . . . . . . . . . . . . 113

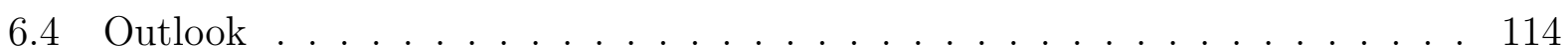

$\begin{array}{ll}\text { BIBLIOGRAPHY } & 115\end{array}$

$\begin{array}{lr}\text { LIST OF APPENDICES } & 122\end{array}$

A RESULTS OF QUAD PLATE ALIGNMENT 123

B BEAM DYNAMICS 140

B.1 Equation of Motion . . . . . . . . . . . . . . . . . . . . 141

B.1.1 Solution to Equation of Motion . . . . . . . . . . . . . . . . . . 141

B.1.2 Courant-Snyder Parameters . . . . . . . . . . . . . . . . . . 142

B.2 Beam Dynamics in g-2 Storage Ring . . . . . . . . . . . . . . . . . . . . . 144

B.2.1 Transfer Matrices . . . . . . . . . . . . . . . . . . . 144

B.2.2 Betatron Amplitudes . . . . . . . . . . . . . . . . . 146

B.2.3 Momentum Dispersion . . . . . . . . . . . . . . . . . . 148

C GEOMETRY FACTOR FOR FAST ROTATION ANALYSIS 152

C.1 Geometry Factor of Square Pulse . . . . . . . . . . . . . . . . . . . . 153

C.2 Geometry Factor of Graziano Pulse . . . . . . . . . . . . . . . . . . . 155

$\begin{array}{ll}\text { VITA } & 161\end{array}$ 


\section{LIST OF TABLES}

1.1 Summary of the SM contributions to the muon anomaly (two values are quoted because of the two recent evaluations of the lowest-order hadronic vacuum polarization). . . . . . . . . . . . . . . . 8

1.2 Measurements of $a_{\mu}$ in history [45]. . . . . . . . . . . . . 11

2.1 General beam requirements and design parameters [12] . . . . . . . . . . 28

2.2 Systematic uncertainty goals related to Tracker for the Muon $g-2$ Experiment

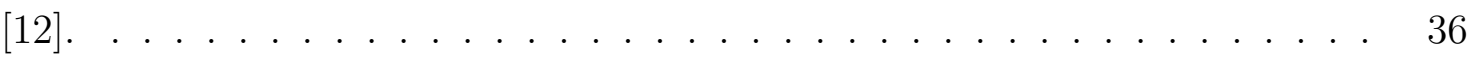

2.3 Event-rate calculation using a bottom-up approach [12]. . . . . . . . . . . 40

2.4 Systematic errors estimated for the anomalous spin precession frequency $\left(\omega_{a}\right)$ measurement. . . . . . . . . . . . . . . . . . . . . . 41

2.5 Systematic errors estimated for the magnetic field $\left(\omega_{p}\right)$ measurement. . . . . 42

3.1 Procedures for recalibration of Sensor CH1. . . . . . . . . . . . . . 48

$3.2 y_{i}$ uncertainties of recalibration part in Fig. 3.7. . . . . . . . . . . . . . 50

4.1 Important frequencies in the muon storage ring (for $n=1.37$ ) [44]. . . . . . 72

5.1 Summary of the muon beam and proton beam at mean radius. . . . . . . . 103 


\section{LIST OF FIGURES}

1.1 The SM of elementary particles: matter fermions in the first three generations, gauge bosons in the fourth column, and the Higgs boson in the fifth. . . . . 2

1.2 Schwinger term: the first order electron self-interaction. . . . . . . . . . . . 4

1.3 Example: Feynman diagrams of QED contributions to $a_{\mu}$ at the tenth order

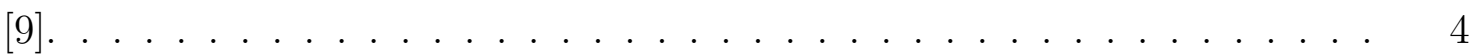

1.4 Example: weak contributions to $a_{\mu}[12] \ldots \ldots \ldots$

1.5 Example: hadronic contributions to $a_{\mu}[12] \ldots \ldots$. . . . . . . . . 6

1.6 Low energy domain $e^{+} e^{-} \rightarrow \pi^{+} \pi^{-}$cross-section [18]. . . . . . . . . . . . . . 7

1.7 Example: hadronic light-by-light contributions to $a_{\mu}[12] \ldots$. . . . . . . 8

1.8 New physics may happen in $g-2: \mathrm{X}$ and $\mathrm{Y}$ stand for new particle (interaction). 9

1.9 The SUSY contributions to $g-2$, and to $\mu \rightarrow e$ conversions, showing the relevant slepton mixing matrix elements. (MDM: magnetic dipole moment; EDM: electric dipole moment) $[12]$. . . . . . . . . . . . . . . . . . . . . 10

1.10 Constraint on large $\tan \beta$ SUSY contributions as a function of $M_{S U S Y}$. The horizontal band shows the $\Delta a_{\mu}=\delta a_{\mu}$. The region left of $M_{S U S Y} \sim 500 \mathrm{GeV}$

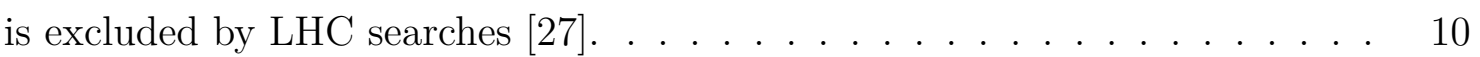

1.11 Pion decay in the Center of Mass frame: $\pi \rightarrow \mu+\bar{\nu} . \quad$. . . . . . . . . . . . 12

1.12 Experimental arrangement of Columbia-Nevis [32] . . . . . . . . . . . . . 12

1.13 Example of Columbia-Nevis experimental results [32] . . . . . . . . . . . 13

1.14 Muon spin and momentum vectors in a magnetic field. . . . . . . . . . . . . 14

1.15 Scheme of the CERN I Muon $g$ - 2 Experiment (1958-1962) [34]. . . . . . . 14

1.16 Arrangement of the CERN II Muon $g-2$ Experiment (1962-1968) [36] . . . 15

1.17 CERN III Muon $g$ - 2 Experiment (1969-1977) [48] . . . . . . . . . . . . 17

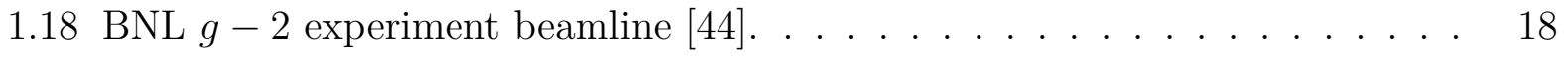

1.19 Overview of the Muon $g-2 /$ EDM Experiment at J-PARC [52] . . . . . . . 19

2.1 BNL E821 citations [58]. . . . . . . . . . . . . . . . . . . . . . 21

2.2 An overview of Fermilab campus (Credit: Fermilab). . . . . . . . . . . . 22

2.3 Feynman diagrams of pion decay. . . . . . . . . . . . . . . . . . . . . . . . . 24

2.4 Rest muon decay: there exists a correlation between the muon spin and decay positron direction. . . . . . . . . . . . . . . . 25 
2.5 Number of decay electrons per unit energy, $N$ or $n(y)$ (arbitrary units), value of the asymmetry $A$ or $a(y)$, and relative figure of merit $N A^{2}$ (arbitrary units) as a function of electron energy $[12] \ldots \ldots \ldots \ldots$

2.6 Muon g-2 beamline: Protons are accelerated in the Linac and Booster. They are re-bunched in the Recycler and then travel through the P1, P2, and M1 lines to the AP0 target hall. Secondary beam then travels through the M2 and M3 lines, around the Delivery Ring, and then through the M4 and M5 lines to the muon storage ring. . . . . . . . . . . . . . . . . . . . . 29

2.7 Timing structure of beam pulse to Muon g-2. . . . . . . . . . . . 30

2.8 Results of simulation for proton bunches: $2.5 \mathrm{MHz}$ voltage curve (upper left), phase space distribution (upper right), phase projection (lower left) and momentum projection (lower right). . . . . . . . . . . . . . . . 31

2.9 Comparison of beam profile (left) with simulation (right): in both profiles, $95 \%$ of the particles captured are contained within 120 ns. . . . . . . . 31

2.10 Overview of the muon storage ring (Credit: Fermilab) . . . . . . . . . . . 32

2.11 Cross section of the muon storage ring magnet [12] . . . . . . . . . . . . 32

2.12 Inflector: view of beam entering into the storage ring. . . . . . . . . 33

2.13 Exit of Inflector: the incident beam center is $77 \mathrm{~mm}$ away from the center of the storage region. The incident muon beam channel is highlighted in red.

2.14 Layout of storage ring, as seen from above, showing the location of Inflector, the Kicker sections (labeled K1-K3), and the quadrupoles (labeled Q1-Q4). . 34

2.15 Kicker Plates for E989. . . . . . . . . . . . . . . . . . . . . 34

2.16 Schematic of the Kicker system. . . . . . . . . . . . . . . . . . . 34

2.17 Electrostatic quadrupole system. . . . . . . . . . . . . . . . 35

2.18 Schematic of Fiber Harp. . . . . . . . . . . . . . . . . . . . . . 36

2.19 Placement of the straw tracker modules. . . . . . . . . . . . . 37

2.20 Schematic of a tracker module. . . . . . . . . . . . . . . . . 37

2.21 Schematic overview of decay positron hitting the calorimeter. $\ldots \ldots$. . . 38

2.22 E821 decay positron arrival time histogram [44] . . . . . . . . . . . . . . . 39

2.23 Histogram of extracted precession frequencies from 1000 simulated $T$-method histograms with and without an exponential gain perturbation of the form $5 \times 10^{-3} \exp \left(-t /\left(\gamma \tau_{\mu}\right)\right)$. The left plot does not include a correction from the simulated laser calibration while the right plot does. . . . . . . . . . . 41

3.1 Schematic view of muon storage ring: ESQ regions are marked as Q1-Q4. . . 44

3.2 Diagram illustrating the ideal orbit, the closed orbit due to non-ideal fields, and betatron oscillations about the closed orbit [70]. . . . . . . . . . 44

3.3 A schematic of the quadrupole cross-section. . . . . . . . . . . . . . . 45 
3.4 The Capacitec used for quad plate survey (left) and the calibration setting for recalibration (right). . . . . . . . . . . . . . . 47

3.5 The readouts of the Capacitec. . . . . . . . . . . . . . . . 47

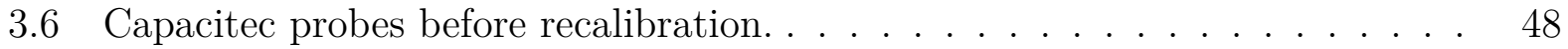

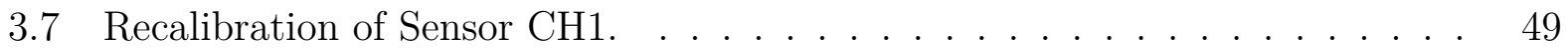

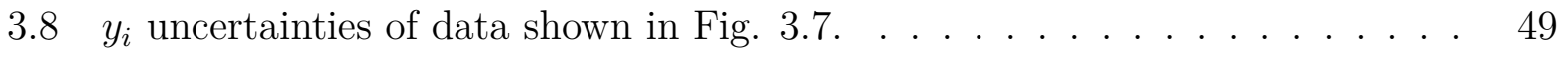

3.9 Three effects of Capacitec calibration. . . . . . . . . . . . . . . . 51

3.10 Calibration: sensor at the center of the plate versus sensor at the edge of the

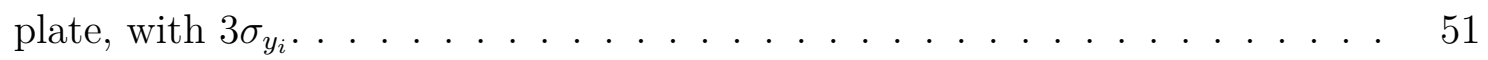

3.11 Calibration with some angle between the sensor surface and the plate surface,

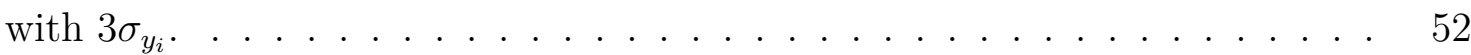

3.12 The scheme of Capacitec Measurement: A stands for the distance between two sensors; B stands for the distance between bottom (inner) sensor and bottom (inner) plate; C stands for the distance between top (outer) sensor and top (outer) plate. The distance between two plates equals $A+B+C$. .

3.13 Quad plate alignment test with Capacitec tool: Blue data is after we perform adjustments, e.g.., adding spacers to the standoff support; Green data is before we perform these adjustments. . . . . . . . . . . .

3.14 Micrometer tools for quad plate alignment: vertical tool (left) for upper/lower plates; radius tool (right) for inner/outer plates. . . . . . . . . . . . . 54

3.15 Spacers used to adjust the quad plate position. . . . . . . . . . . . 55

3.16 Conceptual diagram of about the micrometer tool for vertical electrodes align-

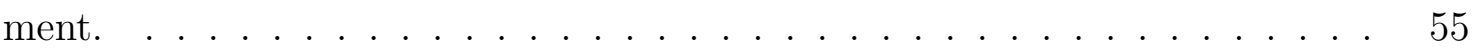

3.17 Cartoon with designed values for vertical quad plate alignment. . . . . . . 56

3.18 S12 (Q4 long) distance between lower base plate and lower quad plate (Green data is before shimming; Blue data is after shimming (final); design value is

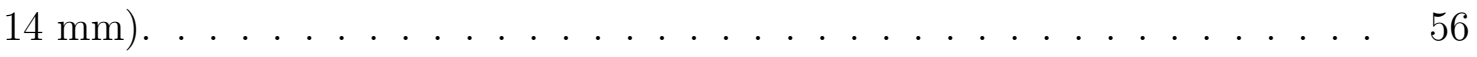

3.19 Micrometer tool for measuring the gap distance. . . . . . . . . . . . 57

3.20 Horizontal quad plate alignment. . . . . . . . . . . . . . . 58

3.21 Laser alignment system for quad plate alignment. . . . . . . . . . . . . . . 59

3.22 Laser scan data for inner and outer plates from H. Friedsam (top view). . . . 59

3.23 Q2L top quad plate deviation distribution: Blue is laser scan data and Red is micrometer tool data. . . . . . . . . . . . . . . . 60

3.24 Q1 outer "Mylar" plate. . . . . . . . . . . . . . . . . . . . . . . 60

3.25 Schematic of Q1 outer plate alignment. . . . . . . . . . . . . . . 61

3.26 Schematic of Q1 outer plate vertical alignment. . . . . . . . . . . . 61

3.27 Example of a "successfully" installed quad extension. . . . . . . . . . . . . . 62 
3.28 Example of installing the connection leads. . . . . . . . . . . . . . . . 63

3.29 Quad Batman (right); Extension tubes (left). . . . . . . . . . . . 63

3.30 Feed-through box. . . . . . . . . . . . . . . . . . . . . . 63

3.31 Preparation of HV resistor. . . . . . . . . . . . . . . . . . . . . . . . 64

3.32 Potting resistors: Vacuum bell jar (left); potted resistors (right). . . . . . . . 64

3.33 Schematic diagram of the scraping and standard HV pulsing systems. . . . . 65

3.34 Time diagram of control pulses. . . . . . . . . . . . . . . . . 66

3.35 Spark detection circuits. . . . . . . . . . . . . . . . 66

3.36 Circuits of control pulses and spark detection with details. . . . . . . . . . 67

3.37 Circuits for pulse count display. . . . . . . . . . . . . . . . 67

3.38 Circuits of control pulses and spark detection with Sten's module. . . . . . . 67

3.39 CAMAC DAQ scheme for spark analysis. . . . . . . . . . . . . . . . 68

4.1 Reference orbit and coordinate system. . . . . . . . . . . . . . . 69

4.2 The dependence of the extracted value of $a_{\mu}$ vs. detector number: (a) With no CBO in the fit function; (b) With CBO in the fit function [12]. . . . . . 73

4.3 A cartoon to understand the CBO: horizontal (radial) CBO is shown in blue. 74

4.4 The Fourier transform to the residuals from a fit to the five-parameter function. 74

4.5 Spin precession from muon $g-2$ only and the additional spin precession with the full CBO [12] . . . . . . . . . . . . . . . . . . . . . . . . 75

4.6 The effect of the $\mathrm{CBO}$ on fitted muon $g-2$ frequency vs. CBO frequency. . $\quad 75$

4.7 Diagram illustrating the detection of a lost muon $\left(\mu^{ \pm}\right)$by finding two-fold or three-fold coincidences between consecutive detectors. . . . . . . . . . . . . 76

4.8 Electric field models from OPERA-2D: 1) XY axis symmetry: straight quadrupole plates; 2) RZ axis symmetry: curved quadrupole plates; 3) plate deformed: Q1 outer plates; 4) plate shifted: misalignment. . . . . . . . . . . . 77

4.9 OPERA-3D geometry model: "short" plates inside a "long" cage and chamber. 79

4.10 Electric potential at arc with different radius. . . . . . . . . . . . . . 80

4.11 Potential at a cylinder surface with "magic" radius and data table. . . . . . 80

4.12 Electric equipotential map of quadrupoles from OPERA-3D with $\pm 27.2 \mathrm{kV}$ on the quadrupole plates and azimuthal average. . . . . . . . . . . . 81

4.13 Example of the field map with one plate charged and the other three plates grounded. . . . . . . . . . . . . . . . . . . . 82

4.14 The tune plane with some resonance lines: E821 ran at $n=0.122,0.137$ and 0.142 , and the possible $n$ values for E989 are 0.142, 0.153, 0.166, 0.175 [91]. .

4.15 Example: quad resonance scan (Stored muons are represented by "Decay Positrons/T0") [95]. 
4.16 Example of fine-grained quad resonance scan (without scraping)-stored muons: represented by "Decay positrons/T0". . . . . . . . . . . . . . . . 86

4.17 Example of fine-grained quad resonance scan (without scraping)-lost muons: represented by "Online Doubles/T0". . . . . . . . . . . . . . . . 86

5.1 Cartoon of beam debunching for fast rotation analysis. . . . . . . . . . . 88

5.2 An overview of the fast rotation signals. . . . . . . . . . . . . . . . . 89

5.3 The distribution of equilibrium radii, as determined from the fast rotation analysis: the dashed curve is obtained from the modified Fourier analysis; and the solid circles are from $\chi^{2}$ minimization method [44] . . . . . . . . . 89

5.4 Example of decay positron count histogram [12] . . . . . . . . . . . . . . . . 90

5.5 Parameterized radial bins of the radial distribution. . . . . . . . . . . . . . 93

5.6 Calculation of $\delta \ldots \ldots \ldots \ldots \ldots$

5.7 Square pulse cell. . . . . . . . . . . . . . . . . . . 94

5.8 Example of Graziano pulse. . . . . . . . . . . . . . . . . . . . . 95

5.9 Injection pulse with a "W" shape used for simulation. . . . . . . . . . . . . . 97

5.10 Decay positron signal seen by the calorimeters. . . . . . . . . . . . . . . . . 97

5.11 Decay positron signal seen by the calorimeters at different time: early time (left) and late time (right) . . . . . . . . . . . . . . . . . . . . . 98

5.12 Toy Monte Carlo model: determination of $t_{0}$ and $T_{C}$. . . . . . . . . . . . 99

5.13 Toy Monte Carlo model: radial distribution. . . . . . . . . . . . . . . . . . 100

5.14 Toy Monte Carlo model: results check at different time scales. Red: decay positron counts seen by calorimeters; Blue: expected counts from fast rotation analysis. . . . . . . . . . . . . . . . . . . . . 100

5.15 Example: gm2ringsim simulation results. Red: decay positron counts seen by calorimeters; Blue: expected counts from fast rotation analysis. . . . . . . 101

5.16 Example: decay positron energy histogram. . . . . . . . . . . . . . . . 102

5.17 Example: decay positron time histogram. . . . . . . . . . . . . . . . . 102

5.18 Example: Fit wiggle plot (ideal signal function) to run 1462 data. . . . . . . 103

5.19 Rebuilt injection pulse shape using the first few bunch pulses (from simulation). 104

5.20 Example: fitting a sine function to extract the $T_{C}$ and $t_{0} \ldots \ldots$. . . . . 104

5.21 Commissioning Run 1462: using "TSpectrum" to determine $t_{0}$ and $T_{C}$ for muon signals. . . . . . . . . . . . . . . . . . . . 105

5.22 Commissioning Run 1462: using "TSpectrum" to determine $t_{0}$ and $T_{C}$ for proton signals. . . . . . . . . . . . . . . . . . . 105

5.23 Example: Fourier Transform of proton signals in run 1462. . . . . . . . . . 105

5.24 Example: new pulse shape rebuilt for fast rotation analysis (commissioning data in summer 2017). . . . . . . . . . . . . . . . . . . . 106 
5.25 Example: radial distribution (commissioning data in summer 2017) . . . . . 106

5.26 Example: T0 pulses of Commissioning for physics run FY18 [99]. . . . . . . 107

5.27 Example: decay positron signal from each bunch at early time. . . . . . . . . 107

5.28 SuperBowlFY18: decay positron signal from bunch \#0 . . . . . . . . . . . 108

5.29 SuperBowlFY18: fitting the wiggle function to the decay positron signal from bunch \#0. . . . . . . . . . . . . . . . . . . . . . . . . . . . . . . . . . . . . . . 108

5.30 SuperBowlFY18: Rebuild pulse shape (not real "injection" shape) for bunch

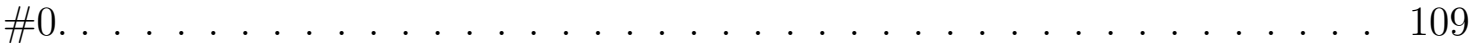

5.31 SuperBowlFY18: muon radius distribution of bunch \#0. . . . . . . . . . . . 109

5.32 SuperBowlFY18: muon radius distribution of each bunch. . . . . . . . . . 109

5.33 SuperBowlFY18: radius distribution for FRA starting at different time points of bunch \#3. . . . . . . . . . . . . . . . . . . . . . . . . . . . . . . . . . . . 110

A.1 Alignment result: Q1 short lower plate. . . . . . . . . . . . . . 124

A.2 Alignment result: Q1 short upper plate. . . . . . . . . . . . . . . 124

A.3 Alignment result: Q1 short inner plate. . . . . . . . . . . . . . . . 125

A.4 Alignment result: Q1 short outer plate. . . . . . . . . . . . . . . . 125

A.5 Alignment result: Q1 long lower plate. . . . . . . . . . . . . 126

A.6 Alignment result: Q1 long upper plate. . . . . . . . . . . . . 126

A.7 Alignment result: Q1 long inner plate. . . . . . . . . . . . . . . . . 127

A.8 Alignment result: Q1 long outer plate. . . . . . . . . . . . . . . . 127

A.9 Alignment result: Q2 short lower plate. . . . . . . . . . . . . 128

A.10 Alignment result: Q2 short upper plate. . . . . . . . . . . . . . 128

A.11 Alignment result: Q2 short inner plate. . . . . . . . . . . . . . . . 129

A.12 Alignment result: Q2 short outer plate. . . . . . . . . . . . . . . . . 129

A.13 Alignment result: Q2 long lower plate. . . . . . . . . . . . . . . . . . 130

A.14 Alignment result: Q2 long upper plate. . . . . . . . . . . . . . . 130

A.15 Alignment result: Q2 long inner plate. . . . . . . . . . . . . . 131

A.16 Alignment result: Q2 long outer plate. . . . . . . . . . . . . . . . . 131

A.17 Alignment result: Q3 short lower plate. . . . . . . . . . . . . . . . . 132

A.18 Alignment result: Q3 short upper plate. . . . . . . . . . . . . . . 132

A.19 Alignment result: Q3 short inner plate. . . . . . . . . . . . . 133

A.20 Alignment result: Q3 short outer plate. . . . . . . . . . . . . . 133

A.21 Alignment result: Q3 long lower plate. . . . . . . . . . . . . . . . . . 134

A.22 Alignment result: Q3 long upper plate. . . . . . . . . . . . . . . . . 134

A.23 Alignment result: Q3 long inner plate. . . . . . . . . . . . . . . 135

A.24 Alignment result: Q3 long outer plate. . . . . . . . . . . . . . 135

A.25 Alignment result: Q4 short lower plate. . . . . . . . . . . . . . . 136 
A.26 Alignment result: Q4 short upper plate. . . . . . . . . . . . . 136

A.27 Alignment result: Q4 short inner plate. . . . . . . . . . . . . 137

A.28 Alignment result: Q4 short outer plate. . . . . . . . . . . . . . . 137

A.29 Alignment result: Q4 long lower plate. . . . . . . . . . . . . . . . . 138

A.30 Alignment result: Q4 long upper plate. . . . . . . . . . . . . . . 138

A.31 Alignment result: Q4 long inner plate. . . . . . . . . . . . . . . 139

A.32 Alignment result: Q4 long outer plate. . . . . . . . . . . . . . . . . 139

C.1 Square pulse case A. . . . . . . . . . . . . . . . . . . 154

C.2 Square pulse case B. . . . . . . . . . . . . . . . . . . 156

C.3 Square pulse case C. . . . . . . . . . . . . . . . . . . . . . . . . . . 157

C.4 Graziano pulse case A. . . . . . . . . . . . . . . . . . . . . . . 158

C.5 Graziano pulse case B. . . . . . . . . . . . . . . . . . . 160 


\section{CHAPTER 1}

\section{INTRODUCTION}

Over the past half century, the Standard Model (SM) has been developed into the most successful theory concerning the fundamental particles and most of their interactions, namely the electromagnetic, weak and strong forces. Not only has it successfully explained almost all the elementary particle experiment results so far, but it has precisely predicted a very wide variety of phenomena, leading us to a better understanding of our universe and the fundamental structure of matter.

Although the SM is considered to be theoretically self-consistent, there are some things that the SM still cannot explain, i.e., the mass of neutrinos, dark matter and dark energy, the asymmetry between matter and antimatter in the universe, and even the most familiar force in our everyday life - gravity. Thus finding physics beyond the SM has become a major effort in particle physics. In this explorative search for New Physics (NP), we can either employ high energy collisions (e.g., experiments at the Large Hadron Collider (LHC)) or make high precision measurements (e.g., the Muon $g-2$ experiment or neutrino experiments).

Among all the searches for NP in particle physics, the intrinsic magnetic moments of fermions play a special and important role. Measurements of the anomalous magnetic moments have provided crucial insights within the field of subatomic structure. The Fermilab Muon $g-2$ Collaboration will measure the muon magnetic moment anomaly to an unprecedented precision level of 0.14 parts per million (ppm) by examining the precession of muons that are subjected to a magnetic field. This will provide a unique and stringent test of the SM of particle physics as well as the physics beyond the SM.

\subsection{The Standard Model of Particle Physics}

The Standard Model of particle physics, formulated in the 1970s, is a theory of fundamental particles and their interactions. It describes three of the four known fundamental interactions, namely the electromagnetic, week and strong forces, in the universe. Gravitational force is not included in the SM. It also classifies all known elementary particles. Its development is based on the quantum theory of fields and it provides the most accurate description of nature at the subatomic level so far. According to the SM, all matter is built from a small number of fundamental spin- $\frac{1}{2}$ particles, called fermions: six quarks and six leptons, which follow Fermi-Dirac statistics; while the carriers of the interactions are characterized as bosons, which possess integer spin (either 0 or 1 ) and follow Bose-Einstein statistics. There are seventeen named particles in the SM, which are organized in Fig. 1.1. The Higgs boson, the last particle in the SM, was discovered in 2012 [1, 2]. Its discovery makes the SM a remarkably successful description of the subatomic world. 


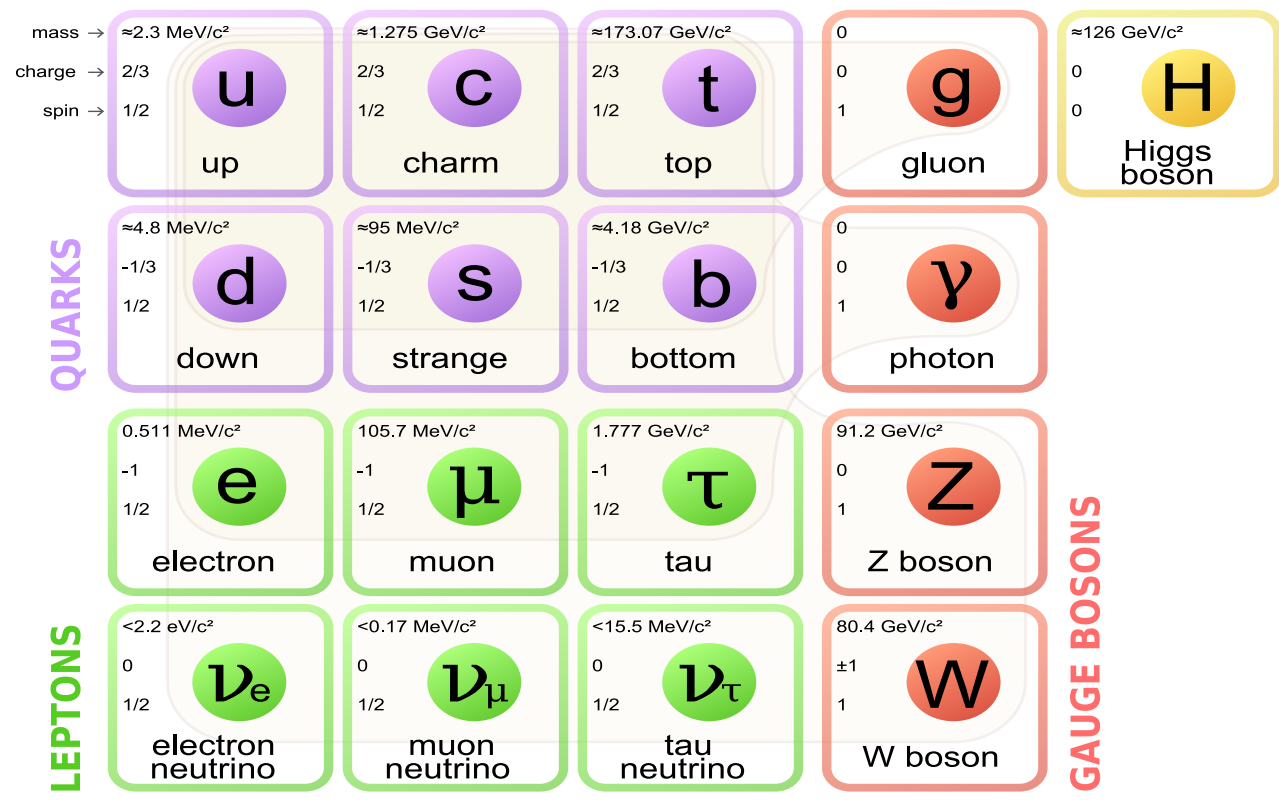

Figure 1.1: The SM of elementary particles: matter fermions in the first three generations, gauge bosons in the fourth column, and the Higgs boson in the fifth.

So far, there are four known fundamental interactions in the universe: the gravitational, electromagnetic, weak and strong interactions. Those interactions work over different ranges and have different strengths. Gravity, acting between all types of particles, is the weakest and it has an infinite range. It is supposedly mediated by exchanging a spin-2 boson graviton, which has not been observed. It is universal and dominant on the scale of the universe but not included in the SM because it is much weaker than the other forces and can be neglected at the level of individual subatomic particles. The electromagnetic interaction acts between all charged particles and is mediated by photon $(\gamma)$ exchange. It also has infinite range but is many times stronger than gravity. The weak and strong interactions are effective only over a very short range and dominate only at the level of subatomic particles. The weak interaction is associated with the exchange of elementary spin-1 bosons between quarks and/or leptons. These mediators are $W^{ \pm}$and $Z^{0}$ bosons, with masses of order 100 times the proton mass. The strong interaction, as its name suggests, is the strongest force of all four fundamental interactions. It is responsible for binding the quarks in the neutron and proton, and the neutrons and protons within nuclei. The strong force is mediated by spin-1, massless particles known as gluons, which couple to color charge, rather like the photons couple to electromagnetic charge.

Fermions are fundamental matter particles in the SM. There are six leptons and six quarks and they can be grouped into three generations. The lightest and most stable particles make up the first generation, whereas the heavier and more unstable particles belong to the second and third generations. Leptons carry integral electric charge. The charged leptons are the electron, muon and tau, while the neutral leptons are the corresponding neutrinos. Neutrinos 
have "flavor" and they are paired with each "flavor" of charged lepton, as indicated by the subscript, i.e., $\left(e, \nu_{e}\right),\left(\mu, \nu_{\mu}\right)$ and $\left(\tau, \nu_{\tau}\right)$. The charged muon and tau are both unstable and decay spontaneously to electrons, neutrinos and other particles. The mean lifetime of the muon is $2.2 \times 10^{-6} \mathrm{~s}$, while that of the tau is only $2.9 \times 10^{-13} \mathrm{~s}$. Neutrinos were postulated by Wolfgang Pauli in 1930 in order to account for the energy and momentum missing in the process of nuclear $\beta$-decay. They experience the weak interactions only. The quarks carry fractional electric charges, of $+\frac{2}{3} e$ or $-\frac{1}{3} e$. The quark "flavor" is denoted by a symbol: $u$ for 'up', $d$ for 'down', $s$ for 'strange', $c$ for 'charmed', $b$ for 'bottom' and $t$ for 'top'. While leptons exist as free particles, quarks are not found to do so. The bound states of quarks are called hadrons, which can be categorized into two families: baryons (made of three quarks) and mesons (made of one quark and one anti-quark). Each quark carries one of the three colors (color charges): $r, g$ and $b$. Quarks are bound together by gluons, which are also colored. Fig. 1.1 shows that the three lepton pairs are exactly matched by the three quark pairs.

\subsection{Anomalous Magnetic Moment}

In particle physics, fermions have intrinsic magnetic fields as well as spin angular momentum. When placed in an external magnetic field, a fermion's internal magnet tends to rotate to align with the external magnetic field. The strength of the internal magnet and the rate of the magnet's gyration determine the fermion's gyromagnetic ratio: $g$. For a lepton $(\ell=e, \mu, \tau)$, the relation between the magnetic dipole moment and the spin is given by

$$
\vec{\mu}_{\ell}=g_{\ell} \frac{Q e}{2 m_{\ell}} \vec{s}, \quad g_{\ell}=2\left(1+a_{\ell}\right), \quad a_{\ell}=\frac{g_{\ell}-2}{2}
$$

where $Q= \pm 1, e>0, m_{\ell}$ is the mass of the lepton and $g_{\ell}$ is the gyromagnetic ratio mentioned above. The small number $a_{\ell}$, called the anomaly (anomalous magnetic moment), arises from quantum fluctuations.

Dirac's quantum theory predicts that $g=2$ for electrons (and for muons) [3]. However, there have been several historical experiments obtaining some discrepancies from Dirac's theory (not just for electrons) [4, 5, 6]. In 1947, Julian Schwinger calculated the famous lowest-order radiative correction to the electron spin magnetic moment and showed that $[7$, 8]

$$
g_{e}=2.00238(6)
$$

This calculation agreed well with experiments. It describes the first order electron selfinteraction, as is shown in Fig. 1.2. This is the start of how we build confidence in new physics models of $g-2$ from a theoretical viewpoint.

\subsection{Muon g-2: Standard Model and New Physics}

In the SM of particle physics, the muon anomalous magnetic moment $a_{\mu}$ arises from the exchange of virtual particles. It can be expressed as a sum of the various gauge sectors where 


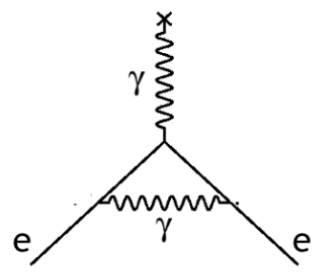

Figure 1.2: Schwinger term: the first order electron self-interaction.
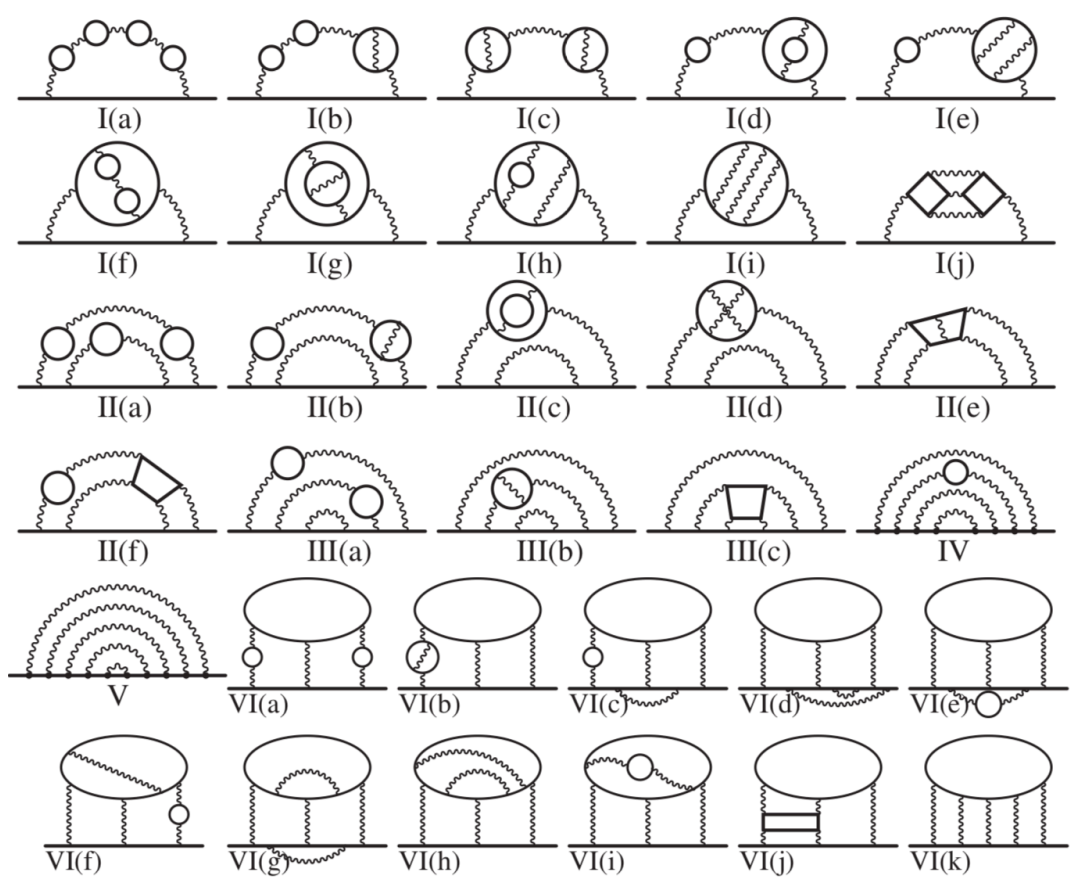

Figure 1.3: Example: Feynman diagrams of QED contributions to $a_{\mu}$ at the tenth order [9]. 
each sector describes one known fundamental force in nature:

$$
a_{\mu}^{S M}=a_{\mu}^{Q E D}+a_{\mu}^{W e a k}+a_{\mu}^{H a d}
$$

\section{QED Contributions:}

The theory of quantum electrodynamics (QED) has been fully developed since the 1940s. QED contributions to $a_{\mu}$ are well understood, and in principle, can be calculated to any desired precision. Each component of the QED contributions can be described by a corresponding Feynman diagram as shown in Fig. 1.3. Those Feynman diagrams can be organized as a sum of exponential series of $\alpha / \pi$ terms:

$$
a_{\mu}^{Q E D}=C_{1}\left(\frac{\alpha}{\pi}\right)+C_{2}\left(\frac{\alpha}{\pi}\right)^{2}+C_{3}\left(\frac{\alpha}{\pi}\right)^{3}+\ldots
$$

where $\alpha \simeq 1 / 137$ is the fine structure constant and $C_{i}(i=1,2, \ldots)$ is constant. $C_{i}$ depends on the lepton mass ratios, i.e., $m_{\mu} / m_{e}$ and $m_{\mu} / m_{\tau}$ and can be obtained by either analytically or numerically. Here, $C_{1}=0.5, C_{2}=0.765857425$ and $C_{3}=24.05050996$ [9].

The most famous and largest contribution was calculated by Schwinger as is shown in Fig. 1.2. Currently, the QED part is calculated up to five loops [9]. The corresponding tenth order self-energy like Feynman diagrams is given in Fig. 1.3. The present QED value is

$$
a_{\mu}^{Q E D}=116584718.951(0.009)(0.019)(0.007)(0.077) \times 10^{-11}
$$

where the uncertainties are from the lepton mass ratios, the eighth-order term, the tenthorder term, and the value of $\alpha$ taken from the ${ }^{87} R b$ atom, $\alpha^{-1}(R b)=137.035999049(90)[10]$.

The muon anomaly $a_{\mu}$ is mainly dominated by QED. However, the calculation of the QED part has reached a precision level of 0.7 parts per billion (ppb), which is very small and can be ignored if we compare it with both the SM error (0.42 ppm) and the Fermilab Muon $g-2$ Experiment measurement goal (0.14 ppm).

\section{Weak Contributions:}

The electroweak contributions to $a_{\mu}$ result from the coupling of a muon to virtual force bosons such as the $W^{ \pm}, Z^{0}$. The leading electroweak contribution diagrams are shown in Fig. 1.4(a) and Fig. 1.4(b). In the approximation where the tiny terms $O\left(m_{\mu}^{2} / M_{W, Z}^{2}\right)$ are neglected, the gauge boson contributions are given by [11]

$$
\begin{aligned}
& a_{\mu}^{\text {Weak }}(W)=\frac{\sqrt{2} G_{F} m_{\mu}^{2}}{16 \pi^{2}} \frac{10}{3} \simeq+388.70(0) \times 10^{-11} \\
& a_{\mu}^{W e a k}(Z)=\frac{\sqrt{2} G_{F} m_{\mu}^{2}}{16 \pi^{2}} \frac{\left(-1+4 s_{W}^{2}\right)^{2}-5}{3} \simeq-193.89(2) \times 10^{-11}
\end{aligned}
$$

Here, $G_{F}=1.1663787 \times 10^{-5} \mathrm{GeV}^{-2}$ is the Fermi coupling constant. The coupling of the photon to the charged $W$ boson is dictated by electromagnetic gauge invariance.

Currently, electroweak contributions have been evaluated using the Higgs mass from CMS and ATLAS up to full two-loop with leading three-loop results [12, 13]. Due to the small 


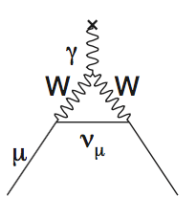

(a)

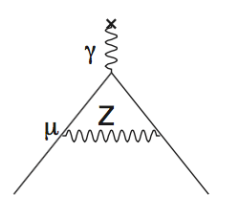

(b)

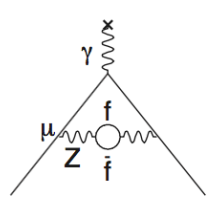

(c)

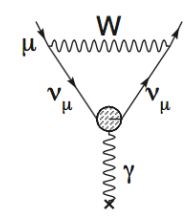

(d)

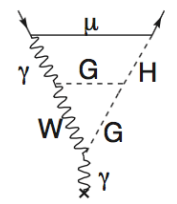

(e)

Figure 1.4: Example: weak contributions to $a_{\mu}[12]$.

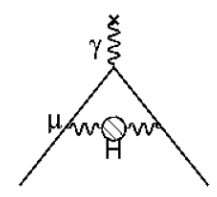

(a)

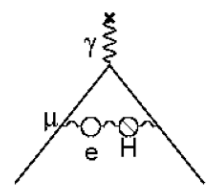

(b)

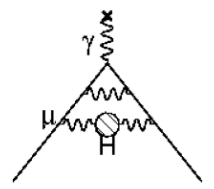

(c)

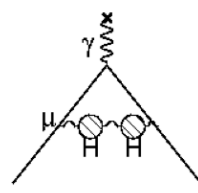

(d)

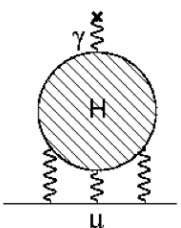

(e)

Figure 1.5: Example: hadronic contributions to $a_{\mu}[12]$.

Yukawa coupling of the Higgs boson to the muon, only the $W$ and $Z$ bosons contribute at a measurable level in the lowest-order electroweak term at the present experiment limits. The total electroweak contribution is

$$
a_{\mu}^{W e a k}=(153.6 \pm 1.0) \times 10^{-11}
$$

where the error comes from hadronic effects in the second-order electroweak diagrams with quark triangle loops, along with unknown three loop contribution $[12,14,15]$.

The Brookhaven Muon $g-2$ Experiment (E821) has been able to test the electroweak contribution to $a_{\mu}$ successfully. The uncertainty of $a_{\mu}^{W e a k}$ is about 60 times smaller than the experimental precision of E821. Therefore, $a_{\mu}^{W e a k}$ can be regarded as known precisely. In fact, the electroweak contribution is almost three standard deviations of the SM, and without it the deviation between theory and the E821 result would be at the $6 \sigma$ level [11].

\section{Hadronic Contributions:}

The hadronic contributions to $a_{\mu}$ come from the coupling of a muon to virtual hadronic particles such as $\pi^{ \pm}$, and $\rho^{ \pm}$. We may obtain the hadronic contributions by replacing the internal lepton loops in the QED by quark loops, adapting charge, color multiplicity and the masses accordingly. However, the quantum field theory (QFT) deals with "single" state particles, like leptons and gauge bosons. Unlike QED and weak contributions, the nonperturbative nature of quantum chromodynamics (QCD) restricts the reliable calculation of hadronic contributions by first principles. Since a single quark state has not been observed, the structure of hadronic particles really relies on the experimental results. Therefore, the uncertainty of $a_{\mu}^{H a d}$ is limited by the experimental uncertainties. So far, it is the largest contributor to the uncertainty of $a_{\mu}^{S M}$. 


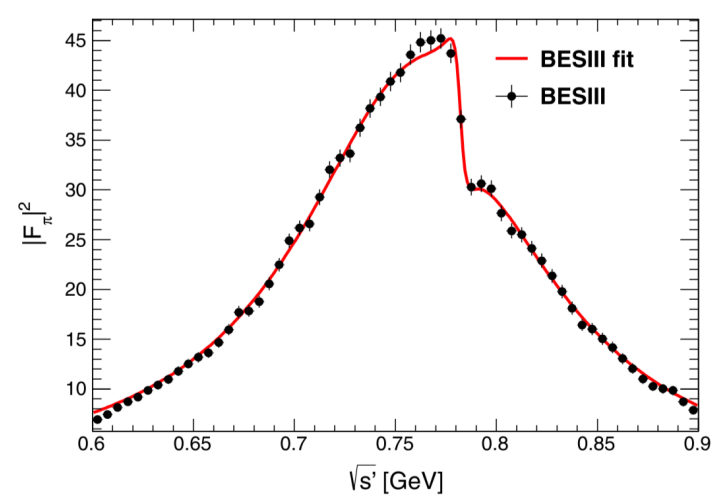

(a) Recent results of low energy domain from BESIII.

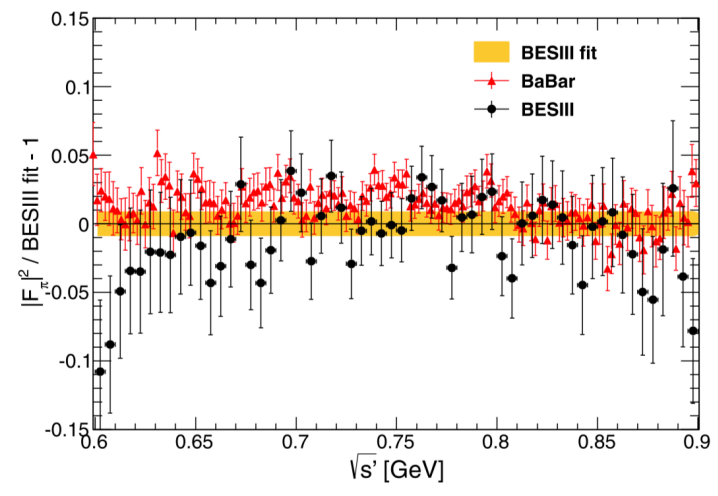

(b) Comparing the data from BaBar with BESIII

Figure 1.6: Low energy domain $e^{+} e^{-} \rightarrow \pi^{+} \pi^{-}$cross-section [18].

Examples of hadronic contributions to $a_{\mu}$ are shown in Fig. 1.5. The first order hadronic contributions come from vacuum polarization as illustrated in Fig. 1.5(a), which refers to a cross-section of $l \bar{l} \rightarrow$ hadrons if split in "half". Using dispersion theory, the first order correction of $a_{\mu}^{\mathrm{Had}}$ can be written as [16]

$$
a_{\mu}^{H a d ; L O}=\left(\frac{\alpha m_{\mu}}{3 \pi}\right)^{2} \int_{4 m_{\pi}^{2}}^{\infty} \frac{d s}{s^{2}} K(s) R(s), \quad \text { where } \quad R(s) \equiv\left(\frac{\sigma\left(e^{+} e^{-} \rightarrow \text { hadrons }\right)}{\sigma\left(e^{+} e^{-} \rightarrow \mu^{+} \mu^{-}\right)}\right)
$$

Here, $K(s)$ is the kinematic factor $[12,17]$. It is only slowly varying in the range of integration and increases monotonically from the $\pi \pi$ threshold $s=4 m_{\pi}^{2}$ to $s=\infty$. The dispersion relation related to the bare cross section measurements of $e^{+} e^{-}$annihilation into hadrons dominates $a_{\mu}^{H a d ; L O}$ at low-energy region (i.e., the $\rho$ and $\omega$ resonances). The $1 / s^{2}$ enhances at low energies, which makes the $g-2$ kernel have very high weight to the low energy range, in particular to the lowest lying resonance $-\rho^{0}$, which shows up in $\pi^{+} \pi^{-} \rightarrow \rho^{0}$ at $m_{\rho} \sim 770$ $\mathrm{MeV}$. This dominance of the low energy hadronic cross-section by a single simple two-body channel provides a possible precise determination of $a_{\mu}$, though a very precise determination of the $\pi^{+} \pi^{-}$cross-section is a rather difficult task. Recent experimental results from BESIII are shown in Fig. 1.6. $R(s)$ is related to the hadronic events and a precise measurement of it requires precise knowledge of the relevant radiative corrections to the hadronic production. At the current level of precision needed, a higher energy region measurement of $R(s)$ may become very important [19].

Another important hadronic contribution to $a_{\mu}$ is called hadronic light-by-light contribution (HLbL) involving four photons coupled to an intermediate hadronic state as is shown in Fig. 1.7. It is the most problematic set of hadronic contributions as the perturbation theory is far from being able to describe the reality for the photon light-by-light scattering. The calculation involves the full rank-four hadronic vacuum polarization tensor $\prod_{\mu \nu \lambda \rho}\left(q_{1}, q_{2}, q_{3}\right)$ including the one-particle reducible pion-exchange pieces [20]. A direct experimental input for the non-perturbative dressed four-photon correlator is not available. One may have to resort to the low energy effective descriptions of QCD like chiral perturbation theory (CHPT) 


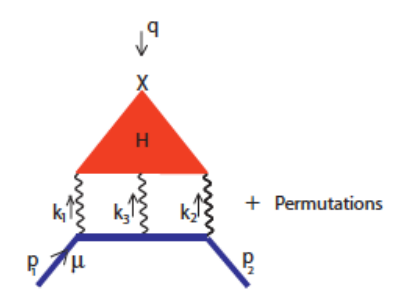

(a)

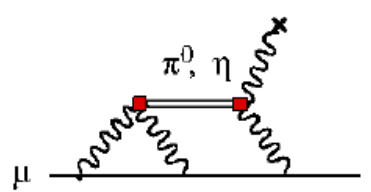

(b)

Figure 1.7: Example: hadronic light-by-light contributions to $a_{\mu}[12]$.

extended to include vector-mesons [11].

\section{A Summary of $a_{\mu}$ SM Value:}

\begin{tabular}{lr}
\hline \hline & VALUE $\left(\times 10^{-11}\right)$ \\
\hline QED $(\gamma+\ell)[9]$ & $116584718.951 \pm 0.009 \pm 0.019 \pm 0.007 \pm 0.077_{\alpha}$ \\
HVP $($ lo $)[21]$ & $6923 \pm 42$ \\
HVP $(l o)[19]$ & $6949 \pm 43$ \\
HVP $($ ho $)[19]$ & $-98.4 \pm 0.7$ \\
HLbL [22] & $105 \pm 26$ \\
EW [13] & $154 \pm 1$ \\
\hline Total SM [21] & $116591802 \pm 42_{\mathrm{H}-\mathrm{LO}} \pm 26_{\mathrm{H}-\mathrm{HO}} \pm 2_{\text {other }}\left( \pm 49_{\text {tot }}\right)$ \\
Total SM [19] & $116591828 \pm 43_{\mathrm{H}-\mathrm{LO}} \pm 26_{\mathrm{H}-\mathrm{HO}} \pm 2_{\text {other }}\left( \pm 50_{\text {tot }}\right)$ \\
\hline \hline
\end{tabular}

Table 1.1: Summary of the SM contributions to the muon anomaly (two values are quoted because of the two recent evaluations of the lowest-order hadronic vacuum polarization).

A summary of the Standard Model $a_{\mu}^{S M}$ predictions is given in Table 1.1 [12]. Among the SM contributions to $a_{\mu}$, the QED parts are the largest contributions but with the smallest uncertainties. Those uncertainties are totally acceptable for the current muon $g-2$ experiment. The electroweak parts are the smallest and their uncertainties can be neglected compared to the required precision of the Fermilab Muon $g-2$ Experiment. The hadronic parts have the largest uncertainties and they require more precise experimental inputs for a better evaluation. The improvement of $a_{\mu}^{H a d}$ will have a significant impact on the muon $g-2$ physics.

\section{Muon $g-2$ New Physics:}

Although the SM theory is very well established and can describe essentially all the current experimental data of laboratory and collider experiments, it is also well established that the SM is not complete and cannot explain a number of fundamental facts, such as gravity, the existence of dark matter and dark energy, the mass of neutrinos and the matterantimatter asymmetry in the universe. Muon $g-2$ describes the coupling of a muon to all 


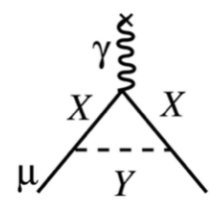

Figure 1.8: New physics may happen in $g-2$ : $\mathrm{X}$ and $\mathrm{Y}$ stand for new particle (interaction).

virtual particles. Therefore, it provides a unique window to search for physics beyond the $\mathrm{SM}$. The new physics (NP) may contribute to $a_{\mu}$ as shown in Fig. 1.8. All the possible new particles or interactions can have an effect on the muon magnetic moment. If a new physics model, with a mass scale $\Lambda$, contributes to the muon mass $\delta m_{\mu}$, it also contributes to $a_{\mu}$. This relation can be expressed as $[12,23]$

$$
a_{\mu}(N P) \sim\left(\frac{m_{\mu}}{\Lambda}\right)^{2} \times\left(\frac{\delta m_{\mu}(N P)}{m_{\mu}}\right)
$$

The NP contributions to muon $g-2$, which we believe must exist, can be part of any measured number. We would be able to tell the SM is incomplete if we can confront an accurately predictable observable with a sufficiently precise measurement. Experimentally, we define the new physics by comparing the $a_{\mu}$ result of the SM value with that measured from experiments such that

$$
a_{\mu}^{N P}=a_{\mu}^{E x p}-a_{\mu}^{S M}
$$

There are many NP models which can contribute to $a_{\mu}$. Most of them are extensions of the SM and predict the NP states: scalars, pseudoscalars, vectors or axial vectors, neutral or charged. Perhaps the simplest one is to add a 4th fermion generation of the sequential fermions where neutrinos can have a large mass as additional light neutrinos have been excluded by LEP. The current bound is $m_{L}>100 \mathrm{GeV}$ [11]. Similarly, there could exist some additional gauge bosons, i.e., $W^{\prime}$ or $Z^{\prime}$. Those additional gauge bosons can mix with the SM gauge bosons and photon. The most promising theoretical scenarios for NP would be the supersymmetric extensions of the SM. Supersymmetry (SUSY) implements a symmetric mapping between fermions and bosons, by changing the spin by $\pm 1 / 2$ units [24].

In the SUSY model, the particle spectrum of the SM is doubled and equipped with an additional Higgs doublet, which might be a natural solution to the Higgs hierarchy problem of the SM. The muon anomaly may be also easily explained in a focus point SUSY scenario. The dominant SUSY contributions can be mediated by a Higgs-gaugino and sneutrino [25]. The typical SUSY contributions to $g-2$ are shown in Fig. 1.9. For a SUSY mass equal to $\Lambda$, the contribution to $a_{\mu}$ is given by

$$
a_{\mu}^{S U S Y} \simeq \operatorname{sign}(\mu) 130 \times 10^{-11} \tan \beta\left(\frac{100 \mathrm{GeV}}{\Lambda}\right)^{2}
$$

where the factor $\tan \beta$ is the ratio of the vacuum expectation values of the two Higgs fields, 


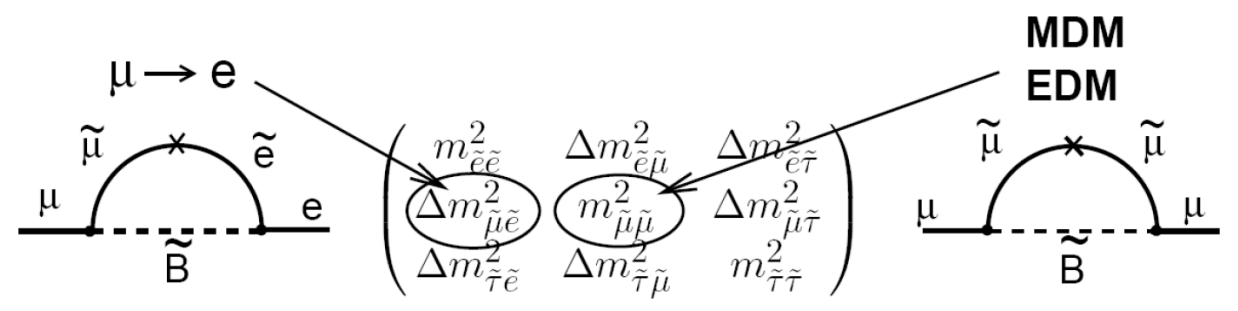

Figure 1.9: The SUSY contributions to $g-2$, and to $\mu \rightarrow e$ conversions, showing the relevant slepton mixing matrix elements. (MDM: magnetic dipole moment; EDM: electric dipole moment) [12].

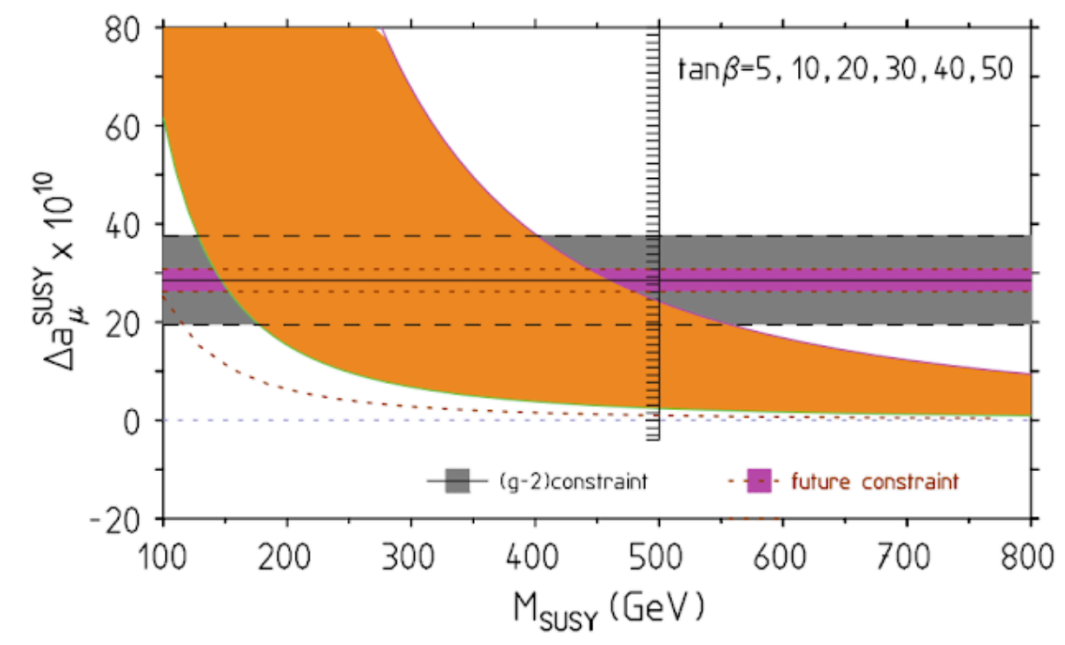

Figure 1.10: Constraint on large $\tan \beta$ SUSY contributions as a function of $M_{S U S Y}$. The horizontal band shows the $\Delta a_{\mu}=\delta a_{\mu}$. The region left of $M_{S U S Y} \sim 500 \mathrm{GeV}$ is excluded by LHC searches [27].

the $\operatorname{sign}(\mu)$ is the SUSY $\mu$-parameter $[11,12,26]$.

Muon $g-2$ will be arguably the most compelling indicator of physics beyond the SM. At the very least, no matter what the agreement between the measured and the SM value turns out to be, it represents a major constraint for speculative new theories such as supersymmetry (SUSY), dark gauge bosons or extra dimensions. An example of the constraint of muon $g-2$ on the larger $\tan \beta$ SUSY contributions as a function of SUSY mass $\left(M_{S U S Y}\right)$ is shown in Fig. 1.10.

Unlike the SUSY particles, which might be very heavy, a light boson can also explain the anomaly of the muon anomalous magnetic moment, such as a hidden (dark) photon and a $Z^{\prime}$ boson $[28,29,30]$. The hidden photon model is one of the simplest models, in which the hidden photon contributes to $a_{\mu}$ at the one-loop level similarly to the SM photon. In presence of a $\mathrm{U}(1)$ symmetry in a hidden sector, the hidden photon couples to the SM particles through a kinetic mixing with the gauge boson of the SM hypercharge. If the 
hidden photon has a mass of $\mathcal{O}(1-100) \mathrm{MeV}$ and a mixing angle of $\mathcal{O}\left(10^{-(2-3)}\right)$, the hidden photon contribution will be sensitive and effective to the current deviation of muon $g-2$ $[28,29]$. Because the hidden photon contributes to $a_{e}$ similarly to $a_{\mu}$ and the SM prediction of $a_{e}$ can be subject to the effect of the hidden photon through the determination of the fine structure constant, the experimental constraints of the hidden photon come from the $a_{e}$ and the hydrogen transition frequencies. Similarly, a $Z^{\prime}$ boson with a mass of $\mathcal{O}(1-100)$ $\mathrm{MeV}$ can couple to the muon and contribute to $a_{\mu}$. The $Z^{\prime}$ boson can have flavor conserving coupling to quarks and leptons and may also allow flavor-changing, e.g., it couples to muons but not electrons. Such a light $Z^{\prime}$ can not only explain the discrepancy in the anomalous magnetic moment of muon, but resolve many other experimental puzzles, such as the proton radius puzzle, the $R_{K}$ puzzle and the gap of high-energy neutrinos in IceCube [30, 31].

\subsection{Muon $g-2$ Experiments in History}

In history, there have been several experiments to execute the measurement of the anomalous magnetic moment of the muon. A summary of past $a_{\mu}$ measurements is given in Table 1.2 .

\begin{tabular}{|r|r|r|r|r|}
\hline \pm & Measurement & $\delta a_{\mu} / a_{\mu}$ & Sensitivity & Reference \\
\hline$\mu^{+}$ & $g=2.00 \pm 0.10$ & & $g=2$ & Garwin et al[32], Nevis (1957) \\
$\mu^{+}$ & $0.00113_{-0.00016}^{+0.00016}$ & $12.4 \%$ & $\frac{\alpha}{\pi}$ & Garwin et al[33], Nevis (1960) \\
\hline$\mu^{+}$ & $0.001145(22)$ & $1.9 \%$ & $\frac{\alpha}{\pi}$ & Charpak et al[34], CERN I (1961) \\
$\mu^{+}$ & $0.001162(5)$ & $0.43 \%$ & $\left(\frac{\alpha}{\pi}\right)^{2}$ & Charpak et al[35], CERN I (1962) \\
\hline$\mu^{ \pm}$ & $0.00116616(31)$ & $265 \mathrm{ppm}$ & $\left(\frac{\alpha}{\pi}\right)^{3}$ & Bailey et al[36], CERN II (1968) \\
\hline$\mu^{+}$ & $0.00106(67)$ & $5.8 \%$ & $\left.\frac{\alpha}{\pi}\right)$ & Henry et al[37], Solenoid (1969) \\
\hline$\mu^{ \pm}$ & $0.001165895(27)$ & $23 \mathrm{ppm}$ & $\left(\frac{\alpha}{\pi}\right)^{3}+$ Hadronic & Bailey et al[38], CERN III (1975) \\
$\mu^{ \pm}$ & $0.001165911(11)$ & $7.3 \mathrm{ppm}$ & $\left(\frac{\alpha}{\pi}\right)^{3}+$ Hadronic & Bailey et al[39], CERN III (1979) \\
\hline$\mu^{+}$ & $0.0011659191(59)$ & $5 \mathrm{ppm}$ & $\left(\frac{\alpha}{\pi}\right)^{3}+$ Hadronic & Brown et al[40], BNL (2000) \\
$\mu^{+}$ & $0.0011659202(16)$ & $1.3 \mathrm{ppm}$ & $\left(\frac{\alpha}{\pi}\right)^{4}+$ Weak & Brown et al[41], BNL (2001) \\
$\mu^{+}$ & $0.0011659203(8)$ & $0.7 \mathrm{ppm}$ & $\left(\frac{\alpha}{\pi}\right)^{4}+$ Weak+? & Bennett et al[42], BNL (2002) \\
$\mu^{-}$ & $0.0011659214(8)(3)$ & $0.7 \mathrm{ppm}$ & $\left(\frac{\alpha}{\pi}\right)^{4}+$ Weak+? & Bennett et al[43], BNL (2004) \\
\hline$\mu^{ \pm}$ & $0.00116592080(63)$ & $0.54 \mathrm{ppm}$ & $\left(\frac{\alpha}{\pi}\right)^{4}+$ Weak+? & Bennett et al[44], BNL (2006) \\
\hline
\end{tabular}

Table 1.2: Measurements of $a_{\mu}$ in history [45].

\section{Columbia-Nevis Experiment:}

The first measurement of the anomaly of muon magnetic moment $\left(a_{\mu}\right)$ was performed in 1957 by R. L. Garwin and his collaborators at the Columbia-Nevis cyclotron [32]. According to the parity violation in weak interactions [46, 47], muons from pion decay are naturally polarized as shown in Fig. 1.11. The longitudinal polarization of the muons offers a natural way to determine the muon magnetic moment.

The arrangement of the experiment is shown in Fig. 1.12. The pion beam with energy around $85 \mathrm{MeV}$ is extracted from the Nevis cyclotron. The beam contains about $10 \%$ muons 


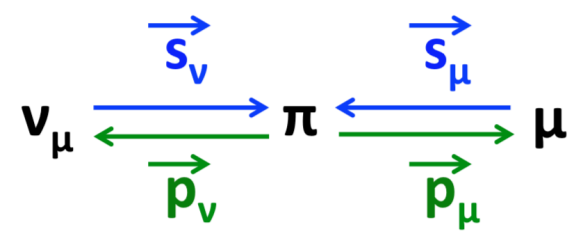

Figure 1.11: Pion decay in the Center of Mass frame: $\pi \rightarrow \mu+\bar{\nu}$.

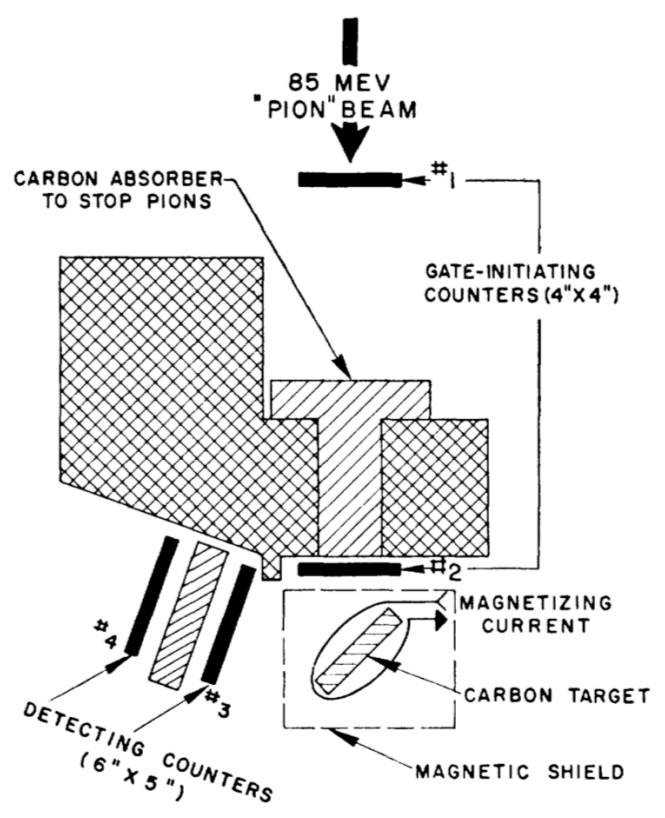

Figure 1.12: Experimental arrangement of Columbia-Nevis [32]. 


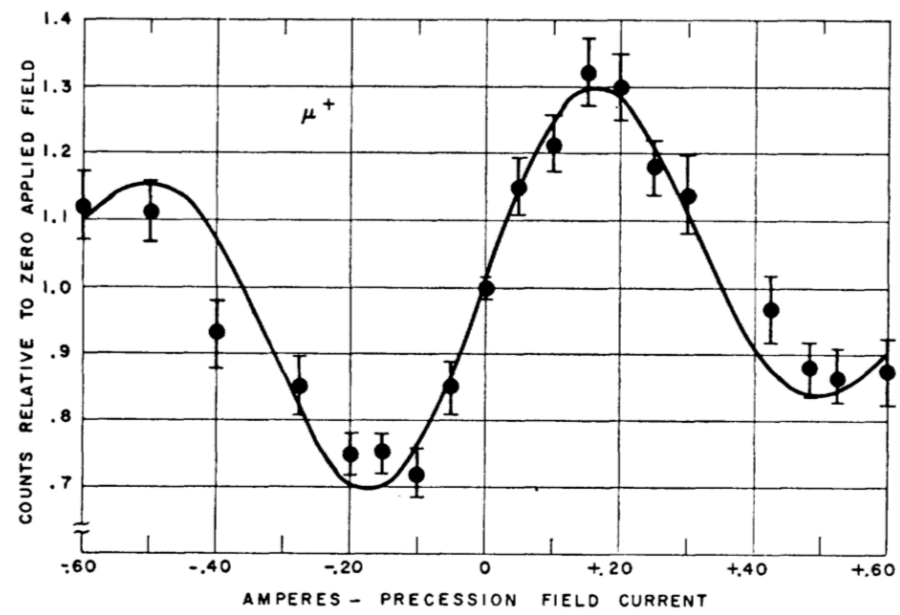

Figure 1.13: Example of Columbia-Nevis experimental results [32].

formed from pion decay-in-flight. Pions will be absorbed by the 8 inches of carbon in the entrance telescope; while the survived muons can be signaled by the two fast "gate-initiating" counters. By applying an external magnetic field, muons will precess. The amount of precession in a fixed time interval is adjusted by the strength of the magnetic field. The decay electron with energy larger than $25 \mathrm{MeV}$ is detected by the two detecting counters in coincidence. The results are presented by plotting counts of decay electrons as a function of magnetizing current for a given time delay as shown in Fig. 1.13.

In the first experimental report [32], the $g$-value for the (free) muon was found to be $2.00 \pm 0.10$, which had a large error, making it hard to elicit the anomaly of the muon magnetic moment. In the subsequent paper of the following upgraded experiment, Garvin et al. reported the anomalous magnetic moment of muon was determined to a precision of $0.007 \%$. With the right muon mass, they concluded that $g_{\mu}=2\left(1.00113_{-0 . .00012}^{+0.00016}\right)[33,45]$. Within such an experimental uncertainty, $a_{\mu}$ is no different from $a_{e}$.

\section{The CERN $g-2$ Experiments:}

From 1958 to 1979, CERN did three experiments to measure the anomaly of the muon magnetic moment. The principles of those three experiments are the same as shown in Fig. 1.14. Basically, a charged muon moving in a uniform magnetic field $B$ will rotate with a cyclotron frequency

$$
\vec{\omega}_{C}=-\frac{q \vec{B}}{m \gamma}
$$

The magnetic field also exerts a torque on the muon's magnetic moment and produces a spin precession frequency

$$
\vec{\omega}_{S}=-\frac{q g \vec{B}}{2 m}-\frac{q \vec{B}}{\gamma m}(1-\gamma)
$$



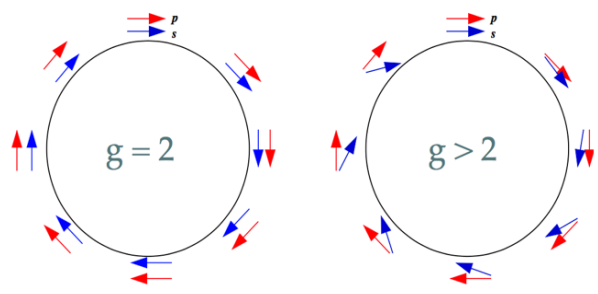

Figure 1.14: Muon spin and momentum vectors in a magnetic field.

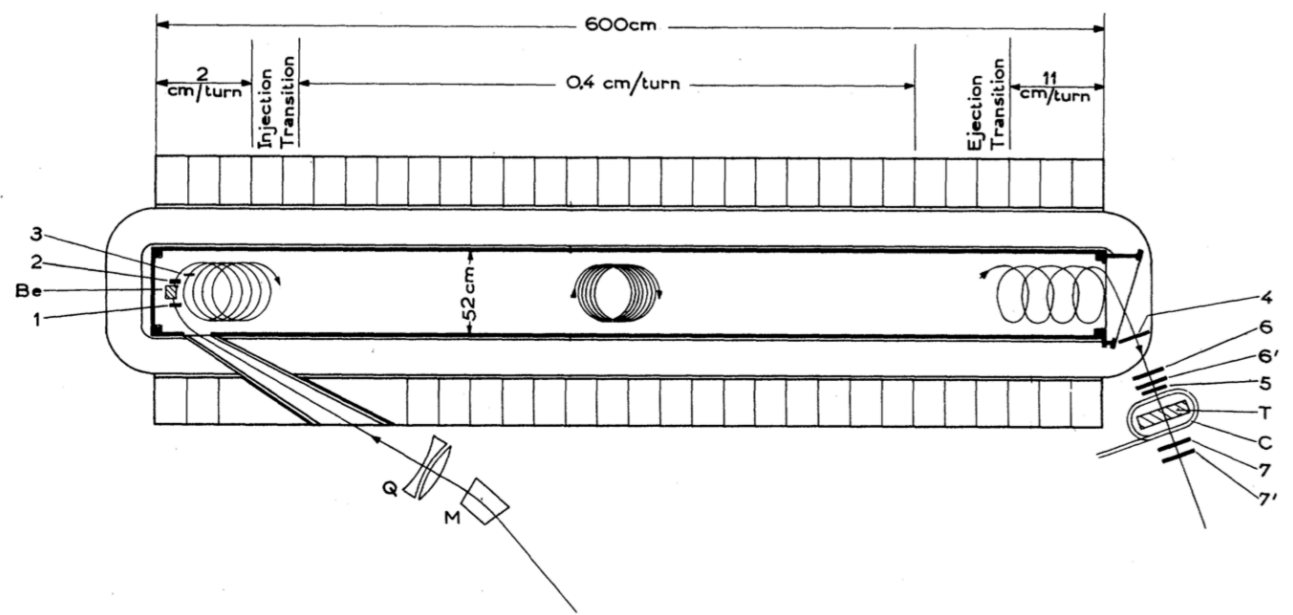

Figure 1.15: Scheme of the CERN I Muon $g$ - 2 Experiment (1958-1962) [34].

The difference between those two frequencies is defined as the anomalous precession frequency

$$
\vec{\omega}_{a}=\vec{\omega}_{S}-\vec{\omega}_{C}=-\left(\frac{g-2}{2}\right) \frac{q \vec{B}}{m}=-a_{\mu} \frac{q \vec{B}}{m}
$$

By measuring the anomalous precession frequency $\vec{\omega}_{a}$ and the magnetic field $\vec{B}$, we can extract the $a_{\mu}$ in Eq. 1.15.

The scheme of the CERN I Muon $g-2$ Experiment is shown in Fig. 1.15. Longitudinally polarized muons formed by forward decay of pions in flight are sent into the entrance channel of the "6-meter magnet". The beam is injected into the solenoid and a stopping beryllium target blocks all the pions. The remaining muons with momentum around $90 \mathrm{MeV}$ travel horizontally in a spiraling orbit from one end of the magnet to the other in a vertical magnetic field $B$, which was not uniform but shimmed carefully to the following form

$$
B(y)=B_{0}\left(1+a y+b y^{2}+c y^{3}\right)
$$

where $B_{0}=1.58 \mathrm{~T}$. The muons emerging from the magnet are stopped in a field free region in the methylene-iodide target. The "backward decay electrons" and "forward decay electrons" are detected by the corresponding spectrometers. The muon spin procession relative to the 


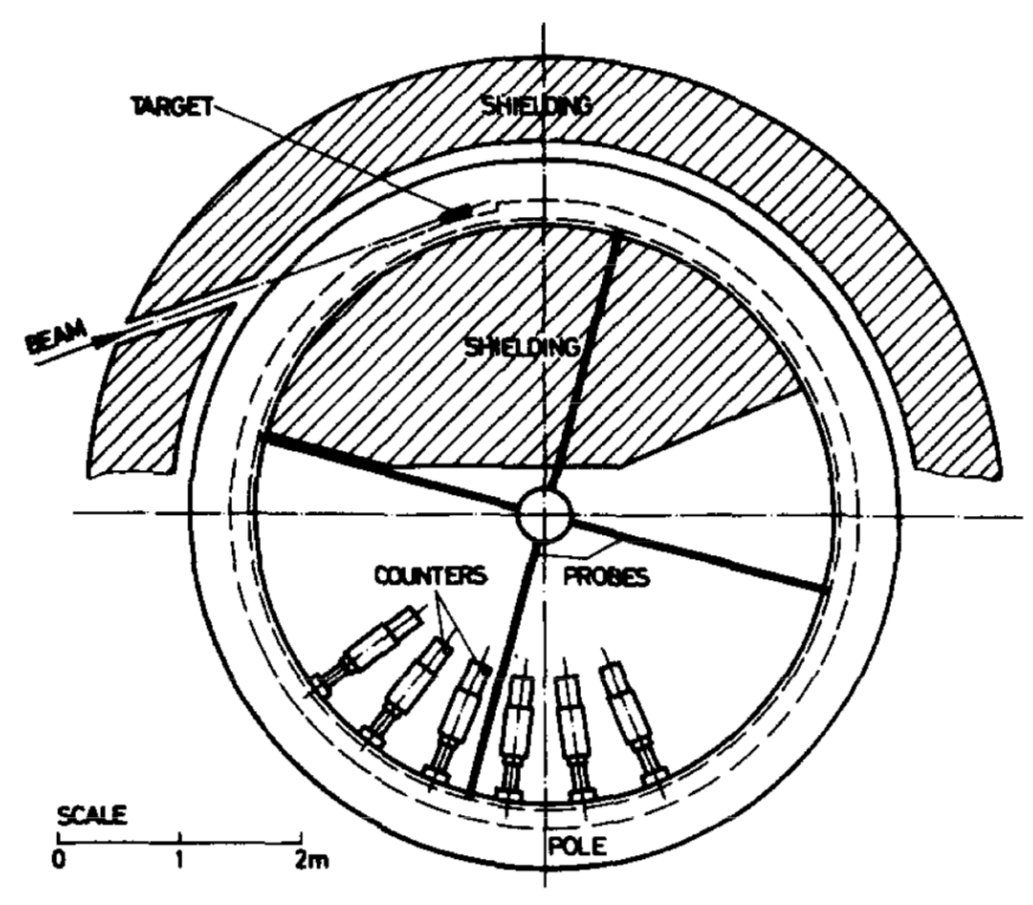

Figure 1.16: Arrangement of the CERN II Muon $g$ - 2 Experiment (1962-1968) [36].

momentum is then determined by the phase of the decay electrons, or in other words, the amount of time spent in the magnet [34]. The measured anomalous magnetic moment for a (free) muon is

$$
a_{\mu}^{E x p}(\mathrm{CERN} \text { I, 1962) }=0.001162(5)
$$

This result agreed with the theoretical value at its level of accuracy and was the first observation of $(\alpha / 2 \pi)$ term in the QED expansion [35].

For the CERN II Muon $g-2$ Experiment, a 5-meter diameter ring magnet with magnetic field $B=1.711 \mathrm{~T}$ was constructed. Protons with energy $10.5 \mathrm{GeV}$ from the CERN Proton Synchrotron enter the ring through a hole in the yoke and hit a target in the magnetic field. Polarized muons (with momentum $p_{\mu}=1.27 \mathrm{GeV} / \mathrm{c}$ ) from the forward decay of pions are stored. The decay electrons from muons emerge on the inside of the ring and produce a large pulse in lead-scintillator-sandwich shower detectors. The polarization directions of the muons are recorded as a function of time by using the decay electrons with the highest energy. The magnetic field is measured in terms of the corresponding mean proton precession frequency $\vec{\omega}_{p}$ by four probes of the nuclear magnetic resonance (NMR) magnetometers located on the muon orbit. The arrangement of the experiment is shown in Fig. 1.16 [36]. A large shielding is introduced to block backgrounds (mostly a huge flux of other hadrons produced when protons hit the target).

The measured anomaly of the muon magnetic moment is

$$
a_{\mu}^{E x p}(\mathrm{CERN} \text { II, 1968) }=0.00116616(31)
$$


which is about 1.8 standard deviations above the theoretical value of $a_{\mu}^{S M}$. The results tested the QED for the muon to the three-loop level.

The CERN III Muon $g-2$ Experiment considered electrostatic focusing to replace the gradient radial magnetic field for vertical focusing in the CERN II experiment. The later one caused a magnetic field variation of $\pm 0.2 \%$ over the muon stored aperture. This destroyed the homogeneity of the magnetic field and introduced a systematic error on the measurement of the average field seen by the muons. In such a case, the extra electric field term should appear in the corresponding cyclotron rotation frequency $\vec{\omega}_{C}$ and spin precession frequency $\vec{\omega}_{S}:$

$$
\vec{\omega}_{C}=-\frac{q}{m}\left[\frac{\vec{B}}{\gamma}-\frac{\gamma}{\gamma^{2}-1}\left(\frac{\vec{\beta} \times \vec{E}}{c}\right)\right]
$$

and

$$
\vec{\omega}_{S}=-\frac{q}{m}\left[\left(\frac{g}{2}-1+\frac{1}{\gamma}\right) \vec{B}-\left(\frac{g}{2}-1\right) \frac{\gamma}{\gamma+1}(\vec{\beta} \cdot \vec{B}) \vec{\beta}-\left(\frac{g}{2}-\frac{\gamma}{\gamma+1}\right)\left(\frac{\vec{\beta} \times \vec{E}}{c}\right)\right]
$$

Using the definition of $a_{\mu}=\frac{g-2}{2}$, the Eq. 1.15 becomes

$$
\vec{\omega}_{a}=-\frac{q}{m}\left[a_{\mu} \vec{B}-a_{\mu}\left(\frac{\gamma}{\gamma+1}\right)(\vec{\beta} \cdot \vec{B}) \vec{\beta}-\left(a_{\mu}-\frac{1}{\gamma^{2}-1}\right) \frac{\vec{\beta} \times \vec{E}}{c}\right]
$$

With the assumption of $\vec{\beta} \cdot \vec{B}=0$, Eq. 1.21 reduces to

$$
\vec{\omega}_{a}=-\frac{q}{m}\left[a_{\mu} \vec{B}-\left(a_{\mu}-\frac{1}{\gamma^{2}-1}\right) \frac{\vec{\beta} \times \vec{E}}{c}\right]
$$

The second term can be removed if a "magic" value of $\gamma \simeq 29.3$ is chose. This idea was adopted by the CERN III experiment and the late muon $g-2$ experiments at Brookhaven National Laboratory and Fermilab. In such a case, the new storage ring $\left(B_{0}=1.5 \mathrm{~T}\right)$ with a radius of $700 \mathrm{~cm}$ was constructed to store muons with a momentum of $3.094 \mathrm{GeV} / \mathrm{c}$ at CERN, as shown in Fig. 1.17. An inflector for direct injection of pions into the ring was used to increase the number of stored muon and reduce the severity of residual hadronic backgrounds in the calorimeter detectors.

The CERN III experiment obtained very high longitudinally polarized muons $(\sim 95 \%)$ and achieved a $a_{\mu}$ measurement precision of $7.3 \mathrm{ppm}$. The results are

$$
a_{\mu}(\mathrm{CERN} \text { III, 1979) }=0.01165924(8.5)
$$

which confirmed the hadronic contributions to the anomaly and established the existence of hadronic vacuum polarization [49].

\section{The Brookhaven Muon $g-2$ Experiment:}

The muon $g-2$ experiment (E821) at Brookhaven National Laboratory (BNL) was started in 1984 with the aim of improving on the CERN III result to a relative precision of 0.35 


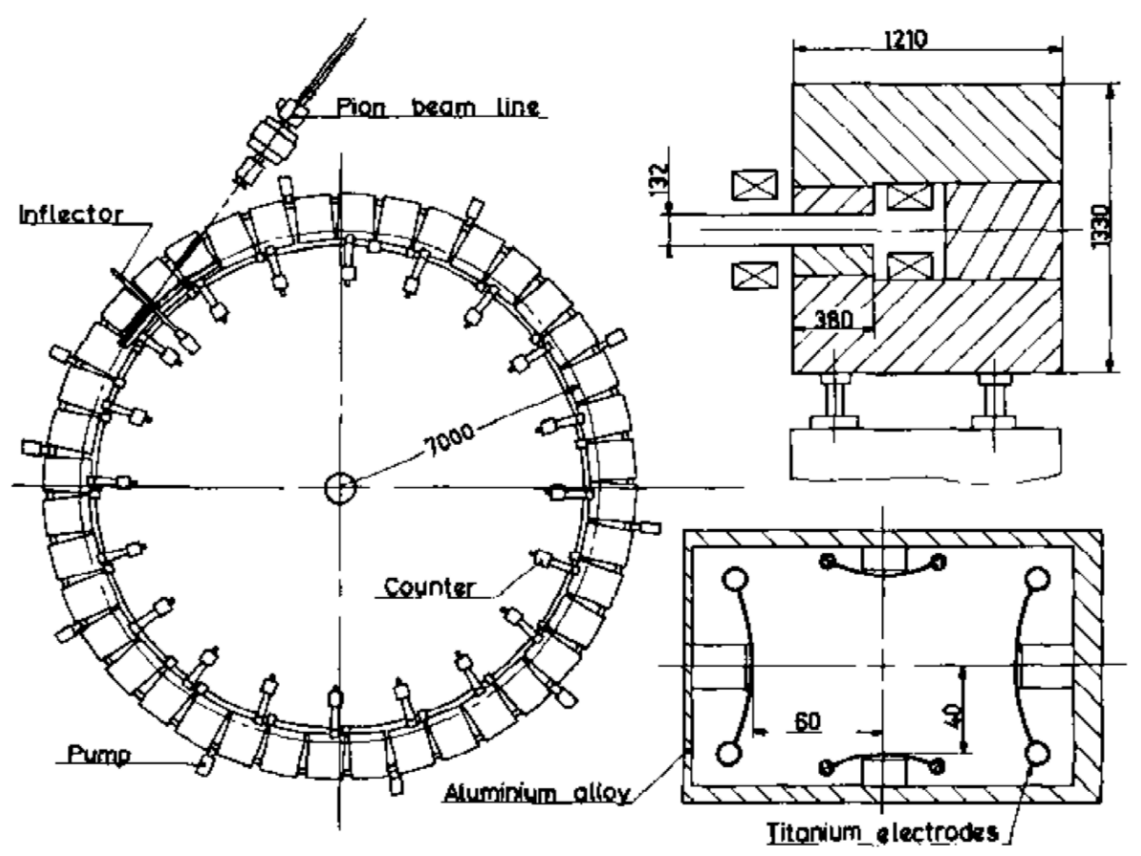

Figure 1.17: CERN III Muon $g$ - 2 Experiment (1969-1977) [48].

ppm. A measurement at such a level of precision can test the electroweak renormalization for the SM and serve as a very sensitive constraint on searching for new physics [45].

The BNL $g-2$ experiment followed the general techniques of the CERN III experiment and added many innovative features. The plan view of the BNL experiment beamline is shown in Fig. 1.18. Protons with energy of $24 \mathrm{GeV}$ are extracted from the BNL Alternating Gradient Synchrotron (AGS) and are focused on a target that is composed of twenty-four 150-mm diameter nickel plates, 6.4-mm thick and separated by $1.6 \mathrm{~mm}$. Pions are collected and transferred into a secondary pion-muon-decay channel. The polarized muons, instead of pions, are directly injected into the storage ring of radius of $711 \mathrm{~cm}$. Instead of a lattice discrete resistive magnet, the ring is a continuous superconducting magnet with a very highly uniform field of $1.45 \mathrm{~T}$. A pulsed kicker system is used to place the muons onto a stable orbit. The electrostatic quadrupoles provide the vertical focusing which permitted the operation at about twice the field gradient of the CERN III experiment. The storage aperture is circular rather than rectangular, in order to reduce the dependence of the average field seen by a muon. The anomalous spin precession frequency is determined from the time histogram of decay electrons signaled in calorimeters. The magnetic field is measured by using an array of NMR probes mounted on a mobile trolley [44].

The BNL $g-2$ experiment reported a final result from a series of precision measurements of $a_{\mu}$

$$
a_{\mu}^{E x p}(\mathrm{BNL}, 2006)=0.00116592080(54)(33)
$$

which had a relative precision level of $0.54 \mathrm{ppm}$ and deviated from the prediction of the SM by about $3.5 \sigma$. This result showed a significant hint of new physics. 


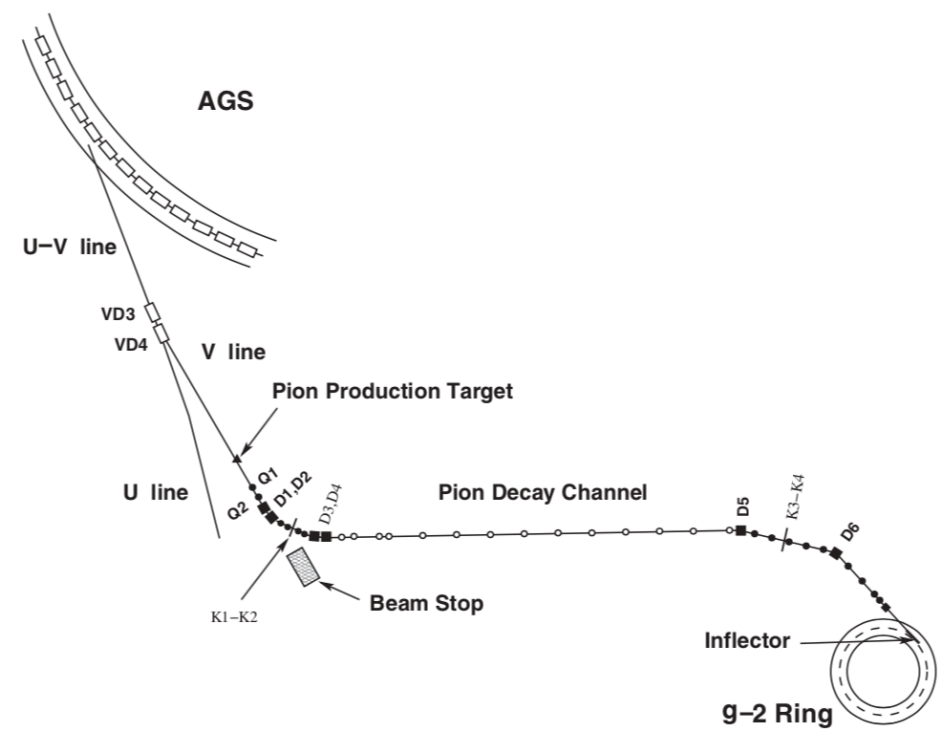

Figure 1.18: BNL $g-2$ experiment beamline [44].

\section{The Fermilab Muon $g-2$ Experiment:}

The Fermilab Muon $g-2$ Experiment (E989) is motivated by the Brookhaven Muon $g-2$ Experiment (E821), which reported a discrepancy of about $3.5 \sigma$ between the measured value and the SM prediction. E989 will measure the anomalous magnetic moment of the muon to a precision of $0.14 \mathrm{ppm}$, which is a factor of four improvement over the measurement of E821. The experiment details will be discussed in Chapter 2.

\section{The Muon $g-2 /$ EDM Experiment at J-PARC:}

The Muon $g-2$ /EDM Experiment at J-PARC (E34) in Japan is proposed. It aims to measure the muon's anomalous magnetic moment and electric dipole moment with ultrahigh precision by utilizing an ultra-cold muon beam from muonium, which is different and independent from methods used for E821 and E989. Currently, parts of the experiment are being tested and developed [50,51,52].

The overview of E34 is shown in Fig. 1.19. A high intensity proton beam with energy of 3 GeV from J-PARC Rapid Cycle Synchrotron is injected to the graphite target and produces pions which will stop in the target. Pions that stop on the surface of the target decay to muons and the generated surface muons are transported to the muonium $\left(\mu^{+} e^{-}\right.$atom $)$ production target. The muonium production is aiming to operate at room temperature (300 $\mathrm{K}$ ), which corresponds to the kinetic energy of $\sim 25$ milli $\mathrm{eV}$ and $2.3 \mathrm{keV} / \mathrm{c}$ in momentum (ultra-cold). The muonium atoms are then ionized by pulsed lasers and eventually produce polarized ultra-cold muons, which will be accelerated up to $300 \mathrm{MeV} / \mathrm{c}$ in a linac. The muons are injected into a storage ring which has a radius of $66 \mathrm{~cm}$ and is about 20 times smaller than the muon storage ring used at BNL and Fermilab. A $3 \mathrm{~T}$ field is applied to store the muon beam. Because the ultra-cold muon beam has an extremely small transverse 


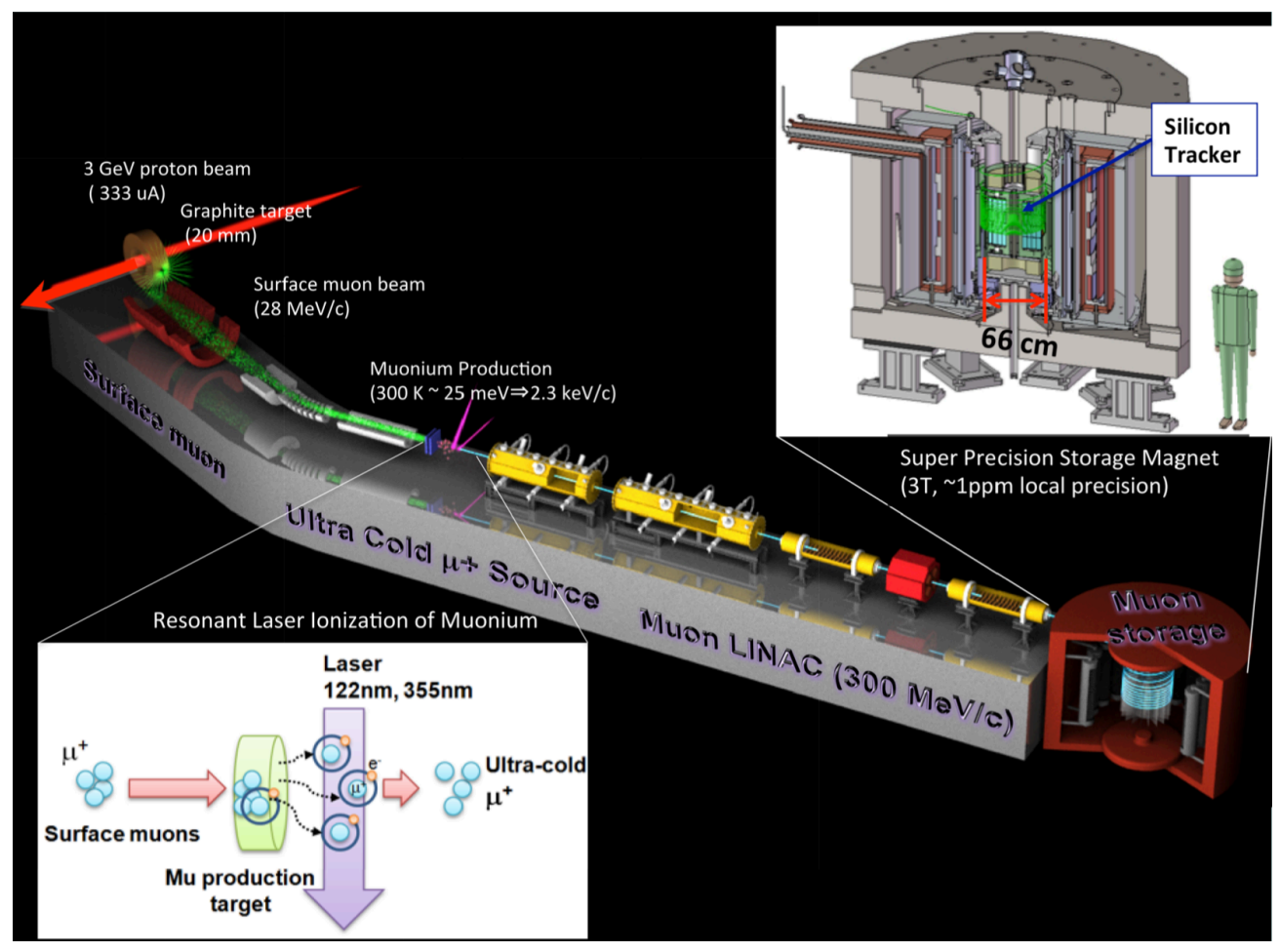

Figure 1.19: Overview of the Muon $g-2 /$ EDM Experiment at J-PARC [52].

momentum, the muon beam can be stored without requiring an electric focusing field. The decay positron will be detected by a silicon strip detector [50].

The E34 experiment at J-PARC has a goal to measure $a_{\mu}$ with a precision of $0.1 \mathrm{ppm}$, which is similar to that of the Fermilab Muon $g-2$ Experiment. It will provide a sensitive test of the SM. 
CHAPTER 2

THE FERMILAB MUON $g-2$ EXPERIMENT (E989)

\subsection{Overview}

As a low-energy observable, the muon anomalous magnetic moment $a_{\mu}$ can be both measured and computed to very high precision. So far, both theory and experiment have reached the sub-part-per-million (sub-ppm) level of precision. $a_{\mu}$ has played an important role in constraining models of physics beyond the Standard Model and will continue to do so in the Large Hadron Collider (LHC) era. In this chapter, we will discuss some details of the Fermilab Muon $g-2$ Experiment, including its motivation, principle, techniques and uncertainties.

\subsubsection{Motivation}

The latest measurement of the muon anomalous magnetic moment $\left(a_{\mu}\right)$ appears to be larger than the expected SM value by more than three standard deviations $(3 \sigma)$. This could be a significant signal of physics beyond the SM and strongly motivates the Fermilab Muon $g-2$ Experiment (E989). The discrepancy between measured $a_{\mu}^{E x p}$ and the SM prediction $a_{\mu}^{S M}$ can be interpreted to point toward several attractive candidates for Standard Model extensions, such as supersymmetry, extra dimensions, or a dark matter candidate [11, 23, $25]$.

The LHC is exploring new physics at the highest energy ranges to date. If the new physics particle has a mass scale of about a $\mathrm{TeV}$, it would be possible to identify it at the current LHC's energy frontier search, although no new physics has yet been found. Energy frontier lepton $[53,54,55]$ and hadron $[56,57]$ colliders may be able to go beyond the LHC. The Muon $g-2$ experiment can carry on the search for new physics using high intensity. The possible candidates, including the large mass scale particles, can interact with the muon and therefore, they are sensitive to the measurement of $a_{\mu}$ as they can contribute to the muon anomaly through the process shown in Fig. 1.8. The current measurement of $a_{\mu}$ is used as a benchmark for new physics, and has been used an input to constrain almost all model dependent searches for physics beyond the SM at the LHC.

BNL E821 has been a very high-impact experiment, with over 3000 citations of its major papers, as shown in Fig. 2.1. However, the current discrepancy between the measurement and the theoretical prediction could be explained as a statistical fluctuation at the threesigma level. A higher precision level of measurement of $a_{\mu}$ is expected to tell us whether this is true or not. The Fermilab E989 experiment has a goal to improve the BNL E821 experimental precision by four-fold, which thereby reduces the error on $a_{\mu}$ to $0.14 \mathrm{ppm}$. If 


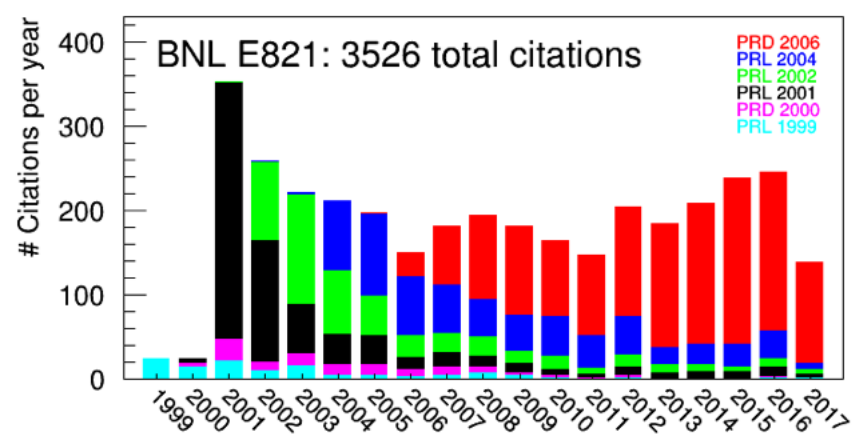

Figure 2.1: BNL E821 citations [58].

the discrepancy measured in BNL E821 is truly an indication of new physics, then such precision-level measurement will differ from the current theoretical SM value by more than the $5 \sigma$ discovery threshold. To achieve such a precision, we need to observe the muon spin precession with more than 20 times the statistics of the BNL E821 while maintaining the systematics at the $100 \mathrm{ppb}$ (part per billion) level.

\subsubsection{Project Scope}

An overview of the Fermilab campus is given in Fig. 2.2, which shows the main Fermilab projects on related protons, neutrinos, muons, etc. Muon g-2 is one of the two muon projects (Muon $g-2$ experiment and Mu2e experiment) being done on the Muon Campus.

The E989 project can be divided into three major areas encompassing the accelerator modifications, storage ring, and detectors. The accelerator portion includes the upgrades and modifications which are required to convert the existing antiproton complex at Fermilab into a muon beam source and deliver a high-purity beam of $3.094 \mathrm{GeV} / \mathrm{c}$ muons to the $g-2$ storage ring. The ring portion includes all of the work needed to reassemble and install the BNL E821 muon storage ring at Fermilab. Many subsystems associated with the injection and storage of muon beam need to be upgraded. The detector portion includes the systems of detectors and electronics that will all be newly constructed to meet the experimental high precision goal.

The Fermilab Muon $g-2$ Experiment is ready for its physical run. A rough time schedule of the Fermilab Muon $g-2$ Experiment is given below (note this might be slightly different from the real schedule):

- Project was initialized and started in 2009;

- Project was approved by Department of Energy (DOE) in September 2012;

- Muon storage ring was transferred to Fermilab in July 2013;

- Full power of the muon storage ring was achieved at Fermilab in 2015;

- Subsystems of the muon storage ring were installed in March 2017; 


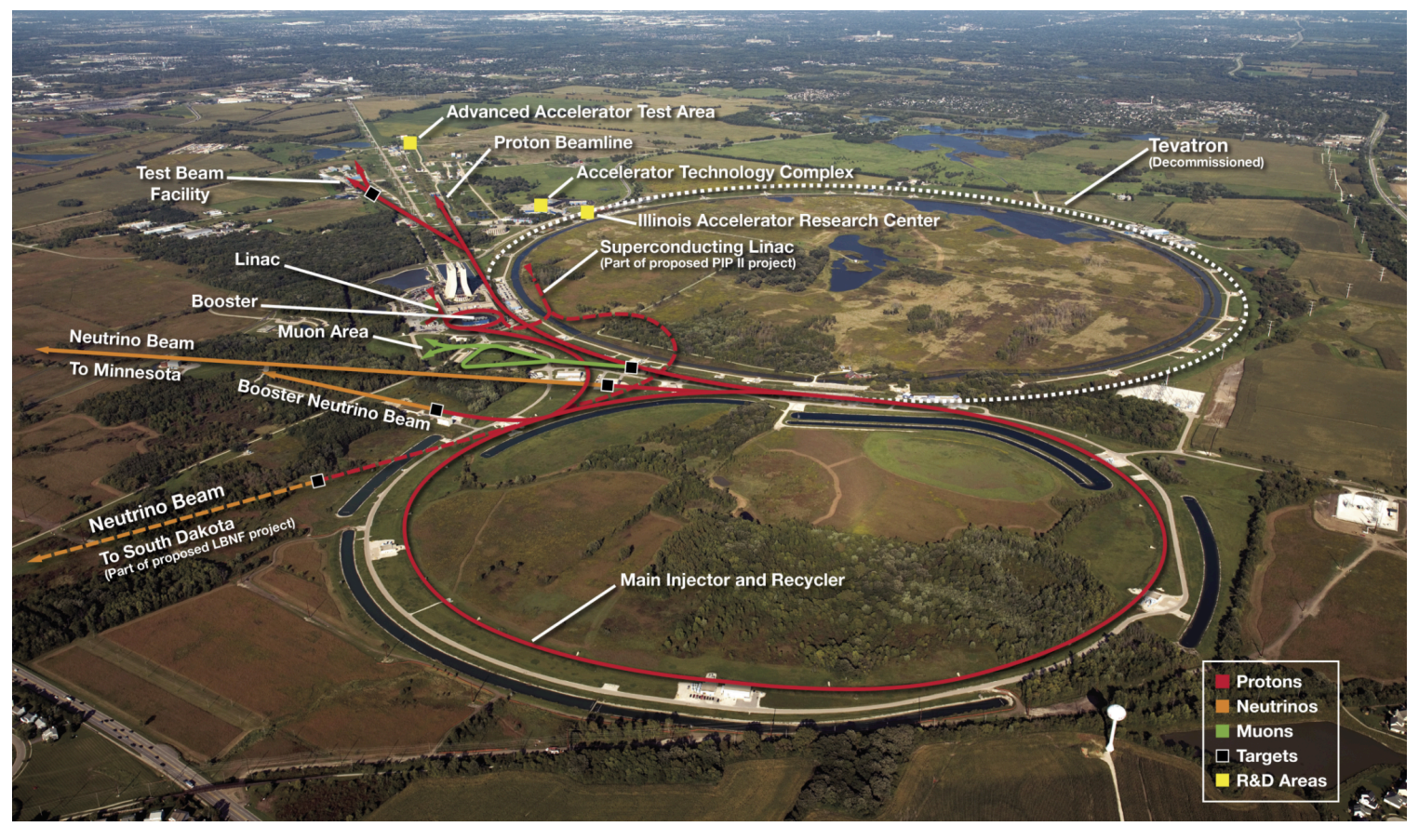

Figure 2.2: An overview of Fermilab campus (Credit: Fermilab).

- Engineering run (commissioning) was successfully carried out in Summer 2017;

- 2-3 times BNL statistics data will be collected in Spring and Summer 2018;

- 5-10 times BNL statistics data will be collected by Summer 2019;

- 20+ times BNL statistics data will be collected by the end of 2020;

- Final report of the project is expected to be published in 2021.

\subsection{Principle}

The measurement of the muon anomalous magnetic moment at Fermilab is based on the same method as the BNL $g-2$ experiment. For a charged muon circulating in a storage ring with static electric field $\vec{E}$ and magnetic field $\vec{B}$, the cyclotron frequency is given by

$$
\vec{\omega}_{C}=-\frac{e}{m}\left[\frac{\vec{B}}{\gamma}-\frac{\gamma}{\gamma^{2}-1}\left(\frac{\vec{\beta} \times \vec{E}}{c}\right)\right]
$$

with the relativistic factors

$$
\vec{\beta}=\frac{\vec{v}}{c}
$$


and

$$
\gamma=\frac{1}{\sqrt{1-\beta^{2}}}
$$

The equation of motion for the spin vector of a muon is referred as Thomas's equation [59]:

$$
\frac{d \vec{s}}{d t}=\frac{e}{m c} \vec{s} \times\left[\left(\frac{g}{2}-1+\frac{1}{\gamma}\right) \vec{B}-\left(\frac{g}{2}-1\right) \frac{\gamma}{\gamma+1}(\vec{\beta} \cdot \vec{B}) \vec{\beta}-\left(\frac{g}{2}-\frac{\gamma}{\gamma+1}\right) \vec{\beta} \times \vec{E}\right]
$$

The spin will rotate at an angular frequency

$$
\vec{\omega}_{S}=-\frac{q}{m}\left[\left(\frac{g}{2}-1+\frac{1}{\gamma}\right) \vec{B}-\left(\frac{g}{2}-1\right) \frac{\gamma}{\gamma+1}(\vec{\beta} \cdot \vec{B}) \vec{\beta}-\left(\frac{g}{2}-\frac{\gamma}{\gamma+1}\right)\left(\frac{\vec{\beta} \times \vec{E}}{c}\right)\right]
$$

The difference between these two frequencies, $\vec{\omega}_{S}-\vec{\omega}_{C}$, is known as the anomalous spin precession frequency, referring to the rate at which the spin precesses relative to the momentum. By using the definition of the muon anomaly $a_{\mu}=(g-2) / 2$, we have

$$
\vec{\omega}_{a}=\vec{\omega}_{S}-\vec{\omega}_{C}=-\frac{q}{m}\left[a_{\mu} \vec{B}-a_{\mu}\left(\frac{\gamma}{\gamma+1}\right)(\vec{\beta} \cdot \vec{B}) \vec{\beta}-\left(a_{\mu}-\frac{1}{\gamma^{2}-1}\right) \frac{\vec{\beta} \times \vec{E}}{c}\right]
$$

In the experiment, we assume that the orbits of muons lie in a plane perpendicular to the magnetic field of the storage ring. Therefore, the second term in Eq. (2.6) may be ignored (a corresponding correction called "pitch-correction" is due to $\vec{\beta} \cdot \vec{B} \neq 0$ ). Experimentally, we could choose a specific $\gamma$ value to let the part of third term $\left(a_{\mu}-\frac{1}{\gamma^{2}-1}\right)=0$ in Eq. (2.6). This will give us a "magic" value $\gamma_{\text {magic }} \simeq 29.3$. The corresponding muon momentum (equilibrium radius of the orbits) is called magic momentum (radius). Therefore, the third term in Eq. (2.6) vanishes. The anomalous spin precession equation reduces to

$$
\vec{\omega}_{a}=-\frac{q}{m} a_{\mu} \vec{B}
$$

Experimentally, we measure $\vec{\omega}_{a}$ using the decay positron signals while measuring the magnetic field $\vec{B}$ by observing the Larmor frequency of stationary protons $\left(\vec{\omega}_{p}\right)$ with the NMR probes. We can solve for $a_{\mu}$ with the two independent measurements.

The purpose of introducing the "magic" momentum is to cancel the term with $\vec{\beta} \times \vec{E}$. However, this is not true even for the situation with a single muon due to the betatron oscillation. In the experiment, we use the electric field to provide vertical focusing to avoid stored muon losses. Muons are injected into the storage ring as a bunch. Thus they have a momentum radial distribution and do not sit on the "magic" radius altogether. The corresponding correction due to the momentum dispersion is called the radial E-field correction and needs to be evaluated in order to achieve our very high precision measurement. 

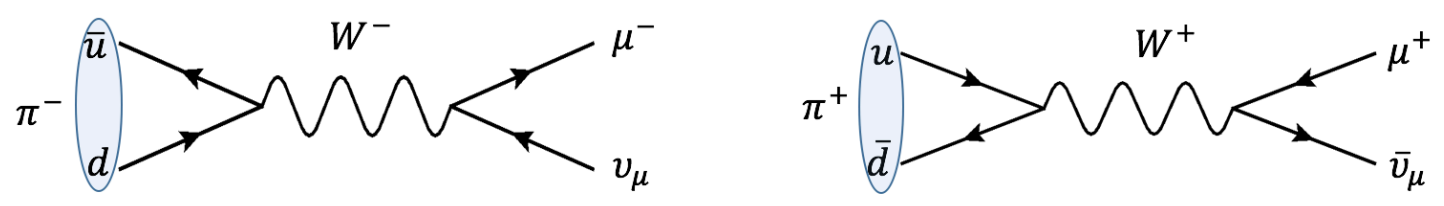

Figure 2.3: Feynman diagrams of pion decay.

\subsubsection{Measurement of $\vec{\omega}_{a}$}

As discussed above, it is necessary to measure $\vec{\omega}_{a}$ in order to measure the muon anomaly. To obtain the muon anomalous spin precession frequency, the muons are needed to be polarized before they are injected into the storage ring. Muons are produced by pion decay. The pion decays via the weak interaction into a muon and a muon neutrino as shown in the Feynman diagrams in Fig. 2.3.

$$
\pi^{-} \rightarrow \mu^{-}+\bar{\nu}_{\mu} \quad \text { or } \quad \pi^{+} \rightarrow \mu^{+}+\nu_{\mu}
$$

The pion is a meson that is made up of two quarks. Its rest mass is about $139.57 \mathrm{MeV}$ and rest mean life is about 26 ns. Because of its light mass, the main possible pion decay processes are to have a muon or an electron together with the corresponding neutrino in the final state. The decay ratio of $\pi^{+} \rightarrow \mu^{+}+\nu_{\mu}$ is [60]

$$
\Gamma\left(\pi^{+} \rightarrow \mu^{+}+\nu_{\mu}\right)=(99.98770 \pm 0.00004) \%
$$

Pion decay is a weak interaction which does not conserve parity. The parity operator $\hat{P}$ performs spatial inversion through the origin such as

$$
\psi^{\prime}(\vec{x}, t)=\hat{P} \psi(\vec{x}, t)=\psi(-\vec{x}, t)
$$

If we apply the parity operator twice, we have

$$
\hat{P} \hat{P} \psi(\vec{x}, t)=\hat{P} \psi(-\vec{x}, t)=\psi(\vec{x}, t)
$$

Therefore, $\hat{P} \hat{P}=I$. If $P$ is an eigenvalue of $\hat{P}, P^{2}=1$ and parity has eigenvalues $P= \pm 1$. For $\pi^{ \pm}$, their spin is 0 and their parity is -1 . For gauge bosons, $P_{W^{ \pm}}=-1$. For spin $1 / 2$ fermions, $P_{\mu^{-}}=P_{\nu_{\mu}}=+1$. For spin $1 / 2$ anti-fermions, $P_{\mu^{+}}=P_{\bar{\nu}_{\mu}}=-1$. Thus, pion decays violate parity.

The other way to understand the parity violation here is through parity transformation. For vectors,

$$
\vec{r} \stackrel{\hat{P}}{\rightarrow}-\vec{r} \quad \text { and } \quad \vec{p} \stackrel{\hat{P}}{\rightarrow}-\vec{p}
$$




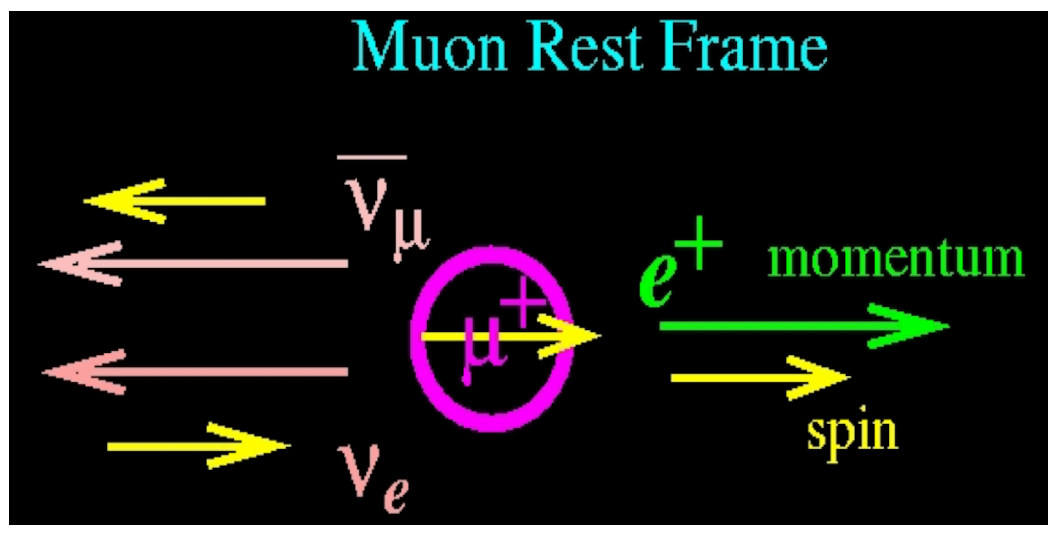

Figure 2.4: Rest muon decay: there exists a correlation between the muon spin and decay positron direction.

For axial vectors,

$$
\vec{L} \stackrel{\hat{P}}{\rightarrow} \vec{L} \quad \text { and } \quad \vec{s} \stackrel{\hat{P}}{\rightarrow} \vec{s}
$$

where $\vec{L}=\vec{r} \times \vec{p}$ is the orbital angular momentum and $\vec{s}$ is the spin angular momentum. In Fig. 1.11, the muon is born polarized in the muon center of mass frame. Because the neutrino (antineutrino) is left-handed (right-handed) and the $\mu^{+}\left(\mu^{-}\right)$is left-handed (righthanded), the helicities (handedness) of the decay $\mu^{+}$and $\nu_{\mu}$ of a $\pi^{+}$with spin 0 are both left-handed.

For positive pion decays, we can select a beam of polarized muons by selecting the muons with the highest energy (a "forward beam") or by selecting the lowest energy muons (a "backward beam"). For the left-handed $\mu^{+}$, its spin vector is anti-parallel to its momentum vector. The "forward beam" are muons with their polarization anti-parallel to their momentum.

As muons circulate around the storage ring, they will decay and the decay positron will be seen by the calorimeters. The measurement of the anomalous spin precession relies on the parity-violating nature of the dominant muon weak decay: $\mu^{+} \rightarrow e^{+}+\nu_{e}+\bar{\nu}_{\mu}$. The pure $(V-A)$ three-body weak decay of muon is "self-analyzing". As shown in Fig. 2.4, the decay positron with the highest energy $\left(E_{\max } \simeq 52.8 \mathrm{MeV}\right)$ travels in an opposite direction to the two neutrinos in the rest muon frame. There exists a correlation between the muon spin and decay positron direction. This correlation allows muon spin direction to be measured as a function of time by applying an energy-cut for the decay positrons.

With the approximation that the energy of decay positron $E \gg m_{e} c^{2}$, the differential decay distribution in the muon rest frame can be written in terms of the fractional decay positron energy $y=E / E_{\max }[61,12]$

$$
\frac{d \Gamma}{d y d \cos \theta d \phi}=\frac{1}{4 \pi}\left(\frac{g_{W}^{2}}{32 m_{W}^{4}}\right) \frac{m_{\mu}^{5}}{192 \pi^{3}}[n(y)][1+a(y) \cos \theta]
$$




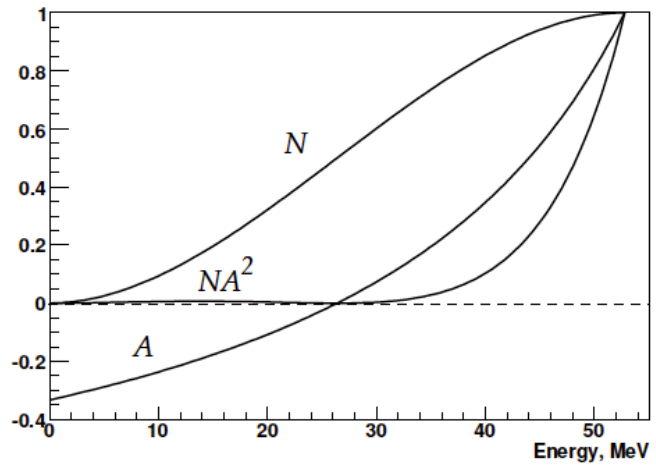

(a) Muon Rest Frame

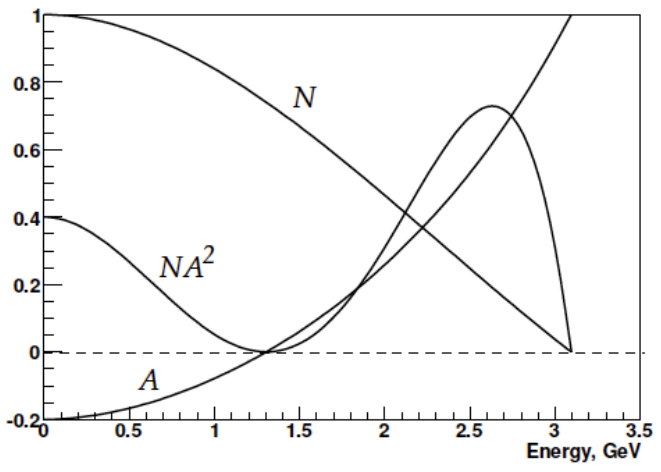

(b) Laboratory Frame

Figure 2.5: Number of decay electrons per unit energy, $N$ or $n(y)$ (arbitrary units), value of the asymmetry $A$ or $a(y)$, and relative figure of merit $N A^{2}$ (arbitrary units) as a function of electron energy [12].

where

$$
n(y)=2 y^{2}(3-2 y) \quad \text { and } \quad a(y)=\frac{1-2 y}{3-2 y}
$$

represent the energy-dependent weighting function and asymmety of the muon decay, as plotted in Fig. 2.5(a). At first glance, we see many more muons decay to positron with $y>0.5$ than with $y<0.5$ and the average asymmetry changes sign at $y=0.5$.

In the lab frame, the muons are traveling with a momentum around $3.094 \mathrm{MeV} / \mathrm{c}$. Eq. (2.15) can be modified under a Lorentz transformation [62]

$$
n(y)=\frac{1}{3}(y-1)\left(4 y^{2}-5 y-5\right) \quad \text { and } \quad a(y)=\frac{1+y-8 y^{2}}{4 y^{2}-5 y-5}
$$

where $y=E / E_{\max }$ is now taken to be the fractional energy of the decay positron relative to the maximum energy in the lab frame, as shown in Fig. 2.5(b). The number of all decay positrons is just a pure exponential function. In the experiment, we employ cuts on the decay positron energy to select those positrons in a range of angles in the muon rest frame. In such a case, $y=\frac{E_{\text {cut }}}{E_{\text {max }}}$. Taking into account the muon's lifetime $\left(\gamma \tau_{0}\right)$ and the anomalous spin precession $\left(\cos \theta \rightarrow \cos \left(\omega_{a} t+\phi\right)\right)$, one expects the number of observed decay positrons above an applied energy threshold $\left(E_{\text {cut }}\right)$ to be

$$
N(t)=N_{0}\left(E_{\text {cut }}\right) e^{-t /\left(\gamma \tau_{0}\right)}\left[1+A\left(E_{\text {cut }}\right) \cos \left(\omega_{a} t+\phi\left(E_{\text {cut }}\right)\right)\right]
$$

Here, the phase $\phi$ stands for the initial spin polarization of the muons. We can extract $\omega_{a}$ by fitting the data of number of decay positrons observed as a function of time. 


\subsubsection{Measurement of the Magnetic Field $\vec{B}$}

To determine $a_{\mu}$, the other parameter that needs to be measured is the magnetic field. Muons are confined to a cylindrical region of $90 \mathrm{~mm}$ diameter inside the storage ring, which sets the scale for the magnetic field measurement. The Fermilab Muon $g-2$ Experiment has a goal to know the magnetic field averaged over running time and the muon distribution to an uncertainty of \pm 70 parts per billion (ppb).

The magnetic field is determined with a pulsed Nuclear Magnetic Resonance (NMR) by observing the Larmor spin precession frequency of a proton in water

$$
\omega_{p}=g_{p}\left(\frac{e B}{2 m_{p}}\right)
$$

where $g_{p}$ is the gyromagnetic factor of the proton. Therefore, we have

$$
B=\frac{2 m_{p} \omega_{p}}{e g_{p}}
$$

The NMR method can be used to measure magnetic fields to absolute accuracies of tens of parts per billion (ppb). It was first developed for E821 and can produce and detect the free induction decay (FID) signals from protons in water. Absolute calibration is important for the field measurement. It is very challenging to effectively transfer the absolute calibration to the many NMR probes required to monitor the field in the large volume and over the long periods of time during which muons are stored.

\subsubsection{Extraction of Muon Anomaly $a_{\mu}$}

Using Eq. (2.7) and Eq. (2.19), we can solve for $a_{\mu}$ :

$$
a_{\mu}=\frac{g_{p}}{2} \frac{m_{\mu}}{m_{p}} \frac{\omega_{a}}{\omega_{p}}=\frac{g_{p}}{g_{\mu}} \frac{m_{\mu}}{m_{p}} \frac{\omega_{a}}{\omega_{p}}\left(1+a_{\mu}\right)
$$

Let's define

$$
\lambda=\frac{g_{\mu}}{g_{p}} \frac{m_{p}}{m_{\mu}}=\frac{\mu_{\mu}}{\mu_{p}}
$$

and

$$
\mathcal{R}=\frac{\omega_{a}}{\omega_{p}}
$$

Hence,

$$
a_{\mu}=\frac{\mathcal{R}}{\lambda-\mathcal{R}}
$$


Here, $\lambda=3.183345137(85)$ is the muon-to-proton magnetic moment ratio measured from muonium (the $\mu^{+} e^{-}$atom) hyperfine structure [63].

We can also recast $a_{\mu}$ in terms of other fundamental constants. Recall that $B=\frac{2 m_{p} \omega_{p}}{e g_{p}}=$ $\frac{\hbar \omega_{p}}{2 \mu_{p}}$ and $\mu_{e}=g_{e} \frac{e \hbar}{4 m_{e}}$. Putting them into Eq. (2.7), we then have

$$
a_{\mu}=\frac{\omega_{a}}{\omega_{p}} \frac{\mu_{p}}{\mu_{e}} \frac{m_{\mu}}{m_{e}} \frac{g_{e}}{2}
$$

The above expression of $a_{\mu}$ will be the one we used in our final analysis [64].

\subsection{Techniques}

In this section, we will give an overview of the experimental techniques and how the experiment is done.

\subsubsection{Production of Polarized Muons}

In order to achieve a statistical uncertainty of $0.1 \mathrm{ppm}$, the total data set should contain at least $1.5 \times 10^{11}$ detected decay positrons with energy greater than $1.8 \mathrm{GeV}$. This means that we need $4 \times 10^{20}$ protons on target including commissioning time and systematic studies. To make sure the detectors perform optimally, the number of protons in a single bunch pulse to the target should be no more than $10^{12}$ (and the number of secondary protons transported into the muon storage ring should be as small as possible). Another issue is that the data acquisition will limit the time between pulses to be at least $10 \mathrm{~ms}$. For muons going around the storage ring, the revolution time is about 149 ns. This requires that the bunch length must be less than that. Moreover, systematic effects on muon polarization limit the momentum spread $d p / p$ of the secondary beam. All of these facts are used to set our beam accelerator parameters, as shown in Table 2.1 [12].

\begin{tabular}{|c|c|c|c|}
\hline Parameter & Designed Value & Requirement & Unit \\
\hline Total proton on target & $2.3 \times 10^{20} /$ year & $4 \times 10^{20}$ & protons \\
\hline Interval betwe & 10 & $\geq 10$ & $\mathrm{~ms}$ \\
\hline Max bunch length (full width) & $120(95 \%)$ & $<149$ & ns \\
\hline Intensity of single 1 & $10^{12}$ & $10^{12}$ & protons \\
\hline Max Pulse $t$ & \pm 10 & \pm 50 & $\%$ \\
\hline$|d p / p|$ of pion & $2-5$ & 2 & $\%$ \\
\hline Momentun & 3.094 & 3.094 & $\mathrm{GeV} / \mathrm{c}$ \\
\hline Muons to ring per $10^{12}$ protons on target & $(0.5-1.0) \times 10^{5}$ & $\geq 6000$ stored & muons \\
\hline
\end{tabular}

Table 2.1: General beam requirements and design parameters [12].

The Fermilab Muon $g-2$ Experiment is designed to take advantage of the infrastructure of the former Antiproton Source, as well as improvements to the Proton Source and the 


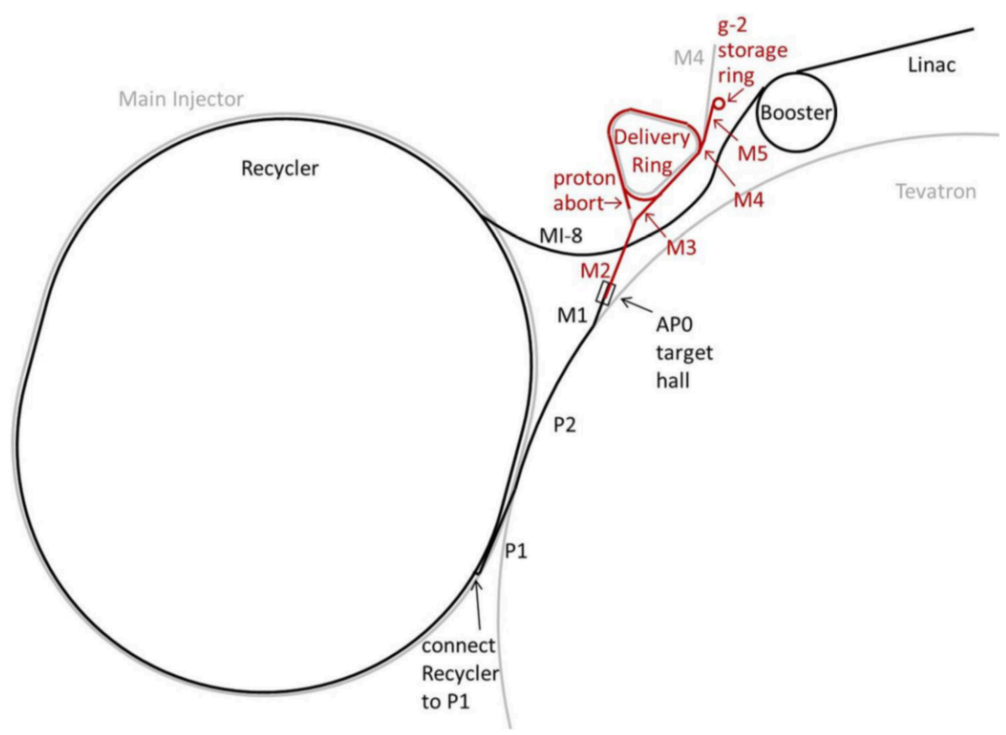

Figure 2.6: Muon g-2 beamline: Protons are accelerated in the Linac and Booster. They are re-bunched in the Recycler and then travel through the P1, P2, and M1 lines to the AP0 target hall. Secondary beam then travels through the M2 and M3 lines, around the Delivery Ring, and then through the M4 and M5 lines to the muon storage ring.

conversion of the Recycler to a proton-delivery machine. The layout of the $g-2$ beamline is shown in Fig. 2.6. Due to the current proton improvement plan, the Booster runs at $15 \mathrm{~Hz}$ with an intensity of $4 \times 10^{12}$ protons per Booster batch. The Main Injector (MI) runs with a 1.333 s cycle time for the $\mathrm{NO} \nu \mathrm{A}$ experiment and twelve batches of beam from the Booster are accumulated in the Recycler and single-turn injected into the MI at the beginning of the cycle. This leaves eight Booster batches available for g-2. Protons from the Booster with $8 \mathrm{GeV}$ kinematic energy are re-bunched into 2 groups of 8 bunches in the Recycler and transported to the target station. Secondary beam with a momentum of $3.11 \mathrm{GeV} / \mathrm{c}$ $( \pm \sim 10 \%)$ is collected from the target and travels through M2 and M3 where muons with momentum of $3.094 \mathrm{GeV} / \mathrm{c}$ from pion decay will be captured. The beam is then injected into the Delivery Ring (DR). All of the pions have decayed into muons after several turns in the DR and muons will also have separated from the heavier protons. Protons are then aborted using a kicker and the muon beam is extracted into the M4 and M5 lines. Finally, the muon beam with momentum of $3.094 \mathrm{GeV} / \mathrm{c}$ will be injected into the muon storage ring. The time structure of the beam pulse is shown in Fig. 2.7 [65].

The beam bunches are spaced by $10 \mathrm{~ms}$ to allow for muon decay and data acquisition in the detector. The longitudinal extent of the bunches is about $120 \mathrm{~ns}$, which is less than the muon revolution time of $149 \mathrm{~ns}$ in the storage ring. The Recycler RF system $(80 \mathrm{kV}$ at 2.5 $\mathrm{MHz}$ ) was designed to achieve those requirements. Simulated $2.5 \mathrm{MHz}$ bunch profiles are shown in Fig. 2.8. A comparison between the measured profile and simulation is shown in Fig. 2.9. Because the voltage is ramped "adiabatically", the phase projection of the bunch profile gets a "W" shape and has tails at each side. The maximum momentum spread is $d p / p= \pm 0.28 \%$. The production rate of $g-2$ will need to accommodate 16 pulses in $1.33 \mathrm{~s}$ 


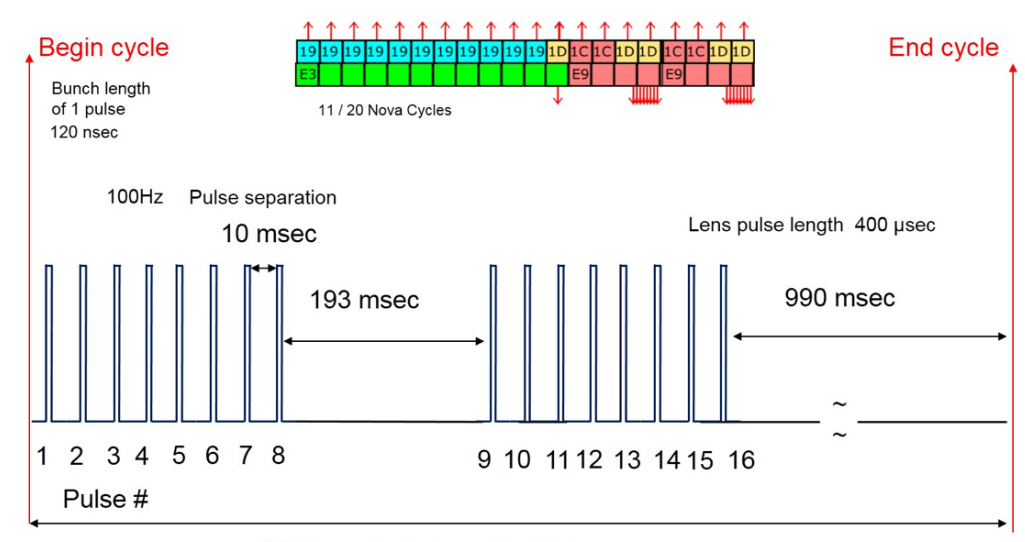

MI Nova Cycle Length $1.33 \mathrm{sec}$

Figure 2.7: Timing structure of beam pulse to Muon g-2.

time cycle with a beam pulse-width of 120 ns. The average pulse rate is $12 \mathrm{~Hz}[12]$.

\subsubsection{Storage of Muons}

The overview of the muon storage ring is shown in Fig. 2.10. It is a continuous superconducting magnet with a C-shaped cross section, as shown in Fig. 2.11. The C-shape will allow the decay electrons to be detected inside the ring. The ring has a radius of 711 $\mathrm{cm}$ and a magnetic field strength of $1.45 \mathrm{~T}$ in the gap. The aperture of the muon beam storage region is a $9.0 \mathrm{~cm}$ circle in the cross-section. The ring is designed to accept muons of the magic momentum within $0.15 \%$. It contains several subsystems to store the muons and meet the experimental measurement goals, such as Inflector, Electrostatic Quadrupoles (ESQ), Kicker, Tracker, etc.

The muon beam enters into the muon storage ring via a hole through the back-leg of the magnet, as shown in Fig. 2.12. Because it enters through the fringe field of the magnet and then into the main field region, it will be strongly deflected unless some other magnet is present to cancel differences. This magnet is called the "Inflector". The beam is injected at an angle of $1.25^{\circ}$ with respect to the tangent line of the central design orbit of the storage ring. The Inflector is $1.7 \mathrm{~m}$ long and it is aligned along this reference line and its downstream end is positioned at the injection point. The point where the reference line is tangent to the storage ring circumference is offset $77 \mathrm{~mm}$ radially outward from the muon central orbit, as shown in Fig. 2.13 [12].

The layout of the storage ring is shown in Fig. 2.14. The muon beam sits $77 \mathrm{~mm}$ offset at the exit of Inflector. If the beam is unperturbed, most muons would strike the Inflector after circulating one turn. Therefore, we have a Kicker system consisting of a series of three identical sectors with $1.27 \mathrm{~m}$ (1.7 m for E821) long parallel plates carrying current in opposite directions, which is located approximately $1 / 4$ of a betatron wavelength around from the Inflector exit. The Kicker system, as shown in Fig. 2.15, will "kick" the beam onto a stable orbit by 10-11 mrad during the first turn (only). The schematic of this is given in Fig. 2.16. 

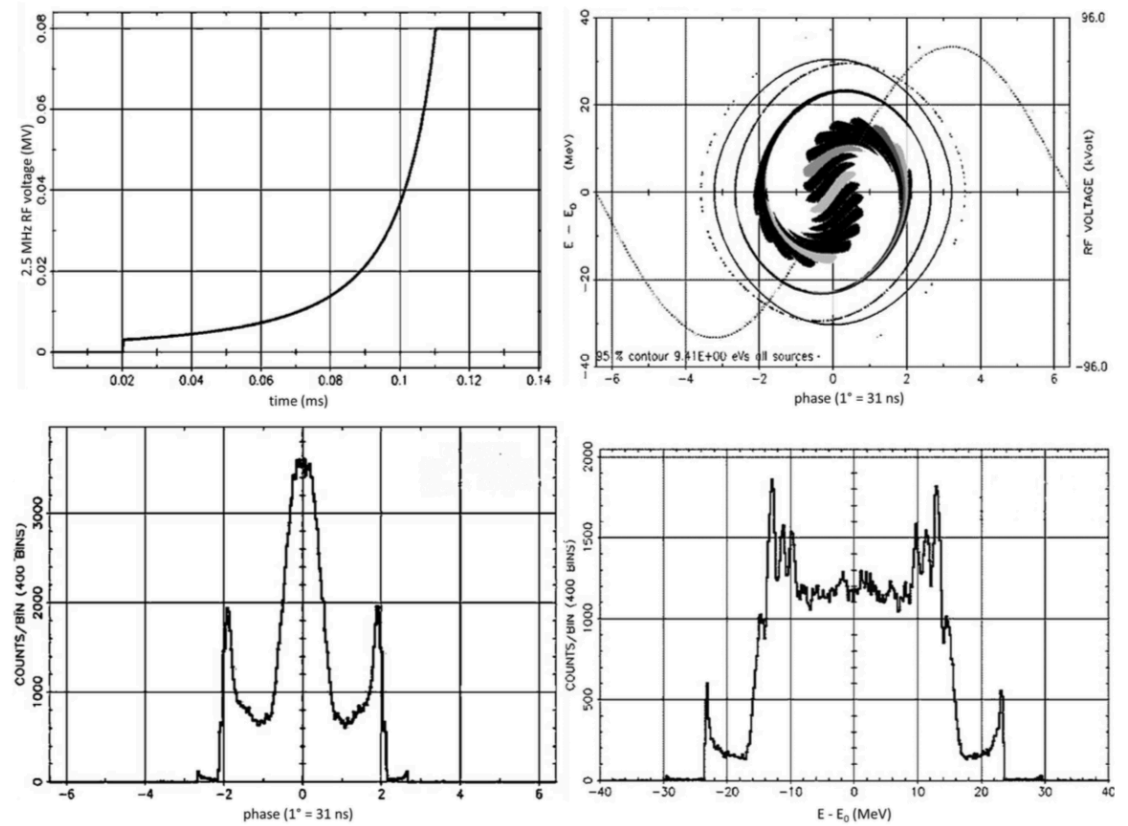

Figure 2.8: Results of simulation for proton bunches: $2.5 \mathrm{MHz}$ voltage curve (upper left), phase space distribution (upper right), phase projection (lower left) and momentum projection (lower right).
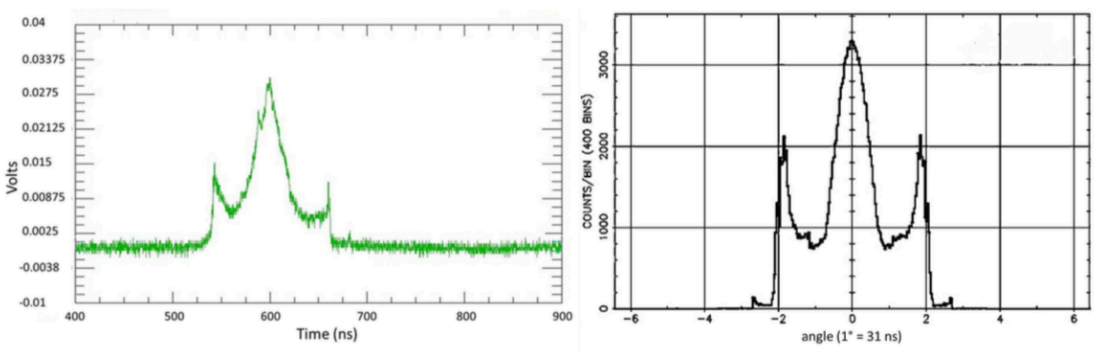

Figure 2.9: Comparison of beam profile (left) with simulation (right): in both profiles, $95 \%$ of the particles captured are contained within $120 \mathrm{~ns}$. 


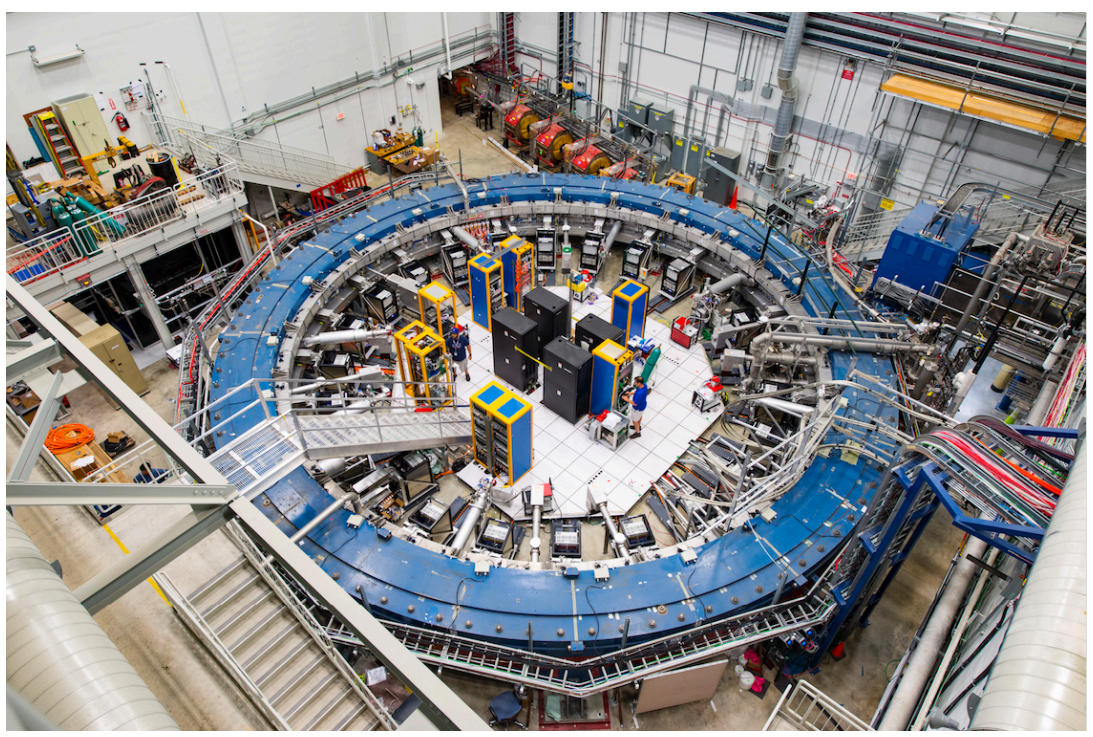

Figure 2.10: Overview of the muon storage ring (Credit: Fermilab).

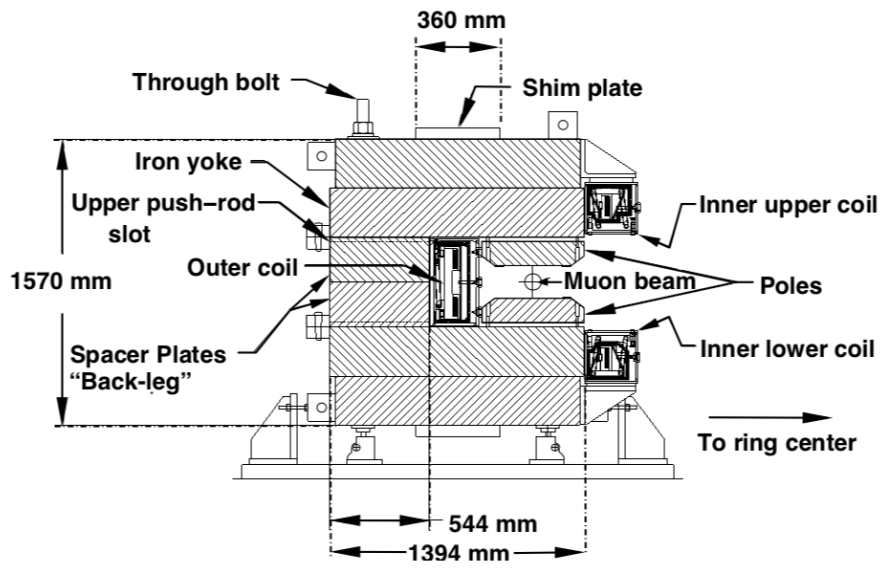

Figure 2.11: Cross section of the muon storage ring magnet [12]. 


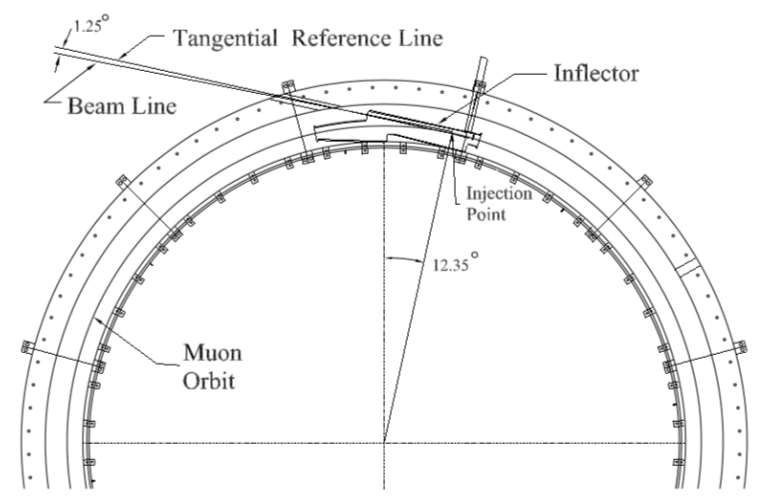

Figure 2.12: Inflector: view of beam entering into the storage ring.

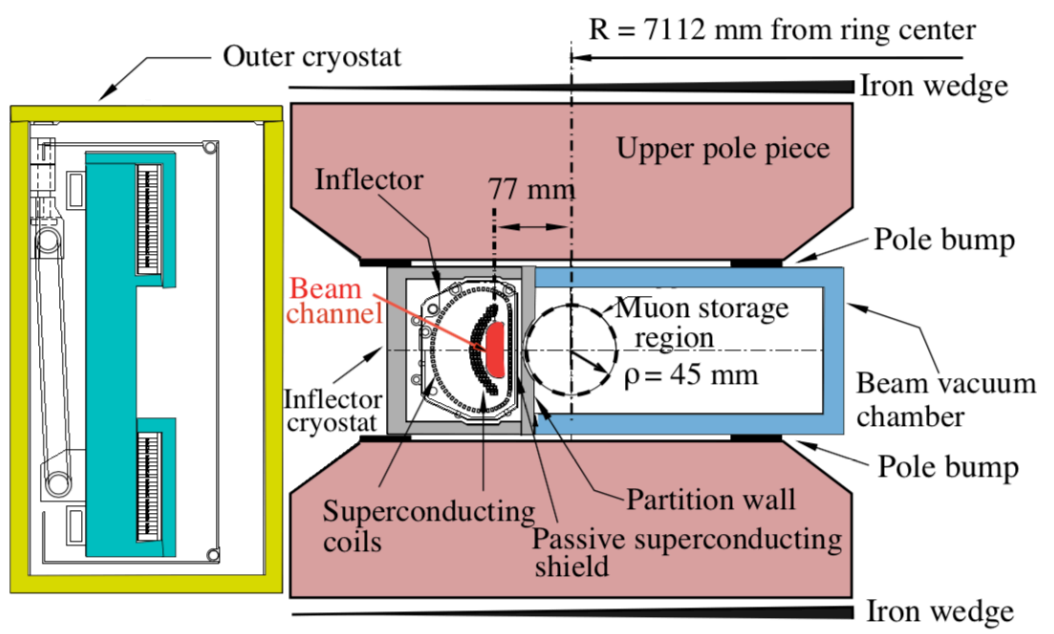

Figure 2.13: Exit of Inflector: the incident beam center is $77 \mathrm{~mm}$ away from the center of the storage region. The incident muon beam channel is highlighted in red. 


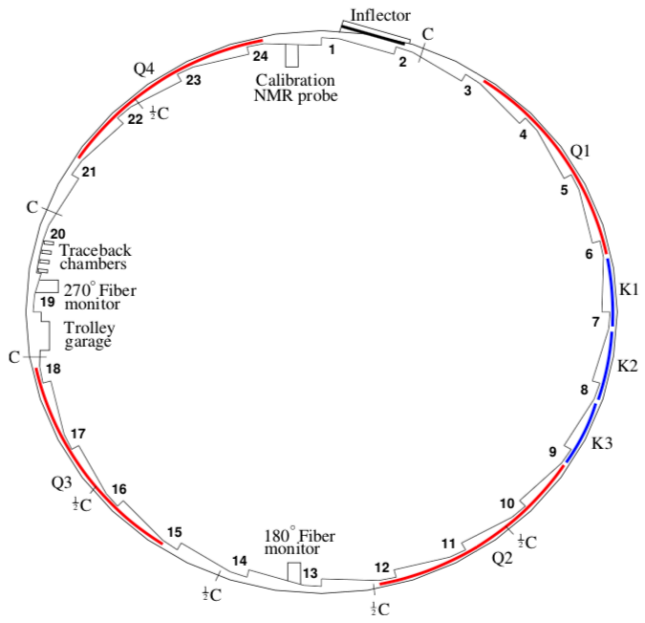

Figure 2.14: Layout of storage ring, as seen from above, showing the location of Inflector, the Kicker sections (labeled K1-K3), and the quadrupoles (labeled Q1-Q4).

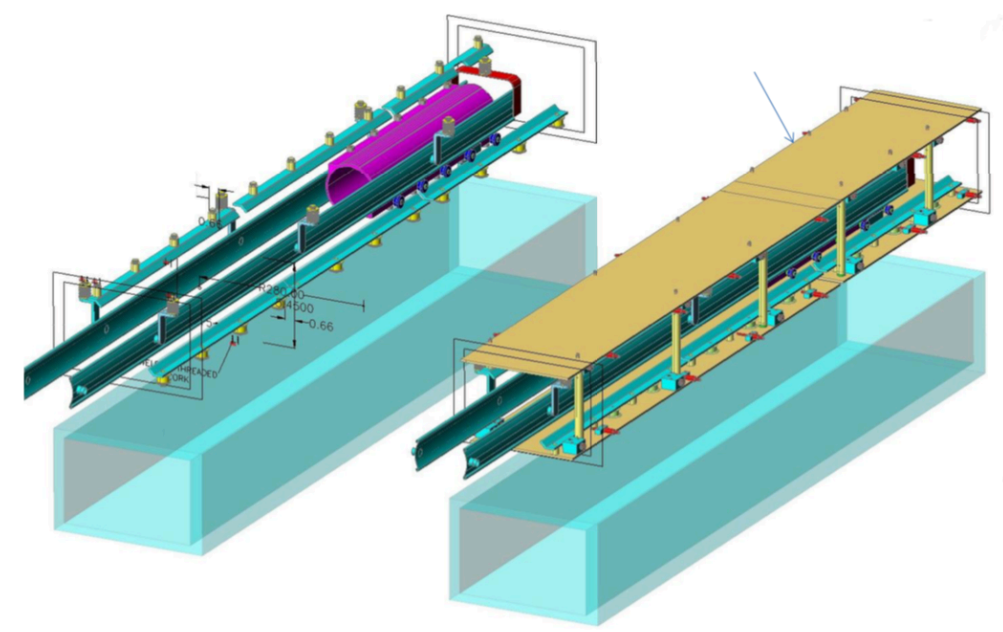

Figure 2.15: Kicker Plates for E989.

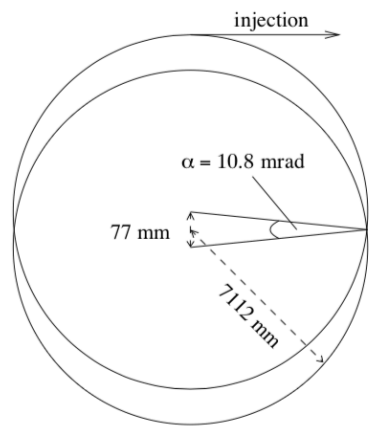

Figure 2.16: Schematic of the Kicker system. 


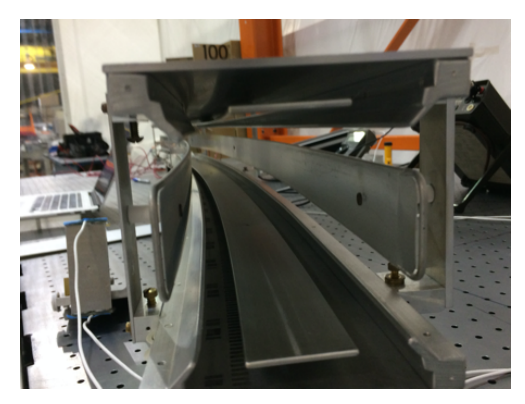

Figure 2.17: Electrostatic quadrupole system.

The Fermilab Muon $g-2$ Experiment has five collimators placed around the storage ring and they define a toroidal volume with a $9 \mathrm{~cm}$ transverse diameter. Once the muon orbit is centered by the kickers, muons will be confined inside the storage region by the magnetic field and the electric field provided by the electrostatic quadrupole system (ESQ). The ESQ, shown in Fig. 2.17, will provide a beam vertical focusing; while the beam horizontal focusing was achieved by the combination of ESQ defocusing and magnetic focusing. Details of ESQ will be discussed in the next chapter. The ESQ field strength is defined by the field index

$$
n=-\frac{R_{0}}{\beta B_{y}} \frac{\partial E}{\partial R}
$$

where $R_{0}$ is the radius of the magic muon orbit, $B_{y}$ is the magnetic field, and $\partial E / \partial R$ is the electric field gradient. As an approximation, the horizontal and vertical betatron oscillation (BO) frequencies can be given by

$$
f_{x_{B O}}=f_{C} \sqrt{1-n} \text { and } f_{y_{B O}}=f_{C} \sqrt{n}
$$

where $f_{C}=c \beta /\left(2 \pi R_{0}\right)$ is the cyclotron rotation frequency.

\subsubsection{Monitoring the Muon Beam}

For our beam dynamics study, we have different subsystems, such as Fiber Harp, Tracker, and Calorimeters, to monitor the muon beam.

The Fiber Harp can be used to directly determine the position (x,y) and angle (x', y') of the beam at injection during commissioning, monitor the evolution of beam properties during the kick and scraping phases, and directly characterize the periodic beam motion. The schematic of Fiber Harp is shown in Fig. 2.18. Each fiber beam monitor holds a "harp" of seven scintillating fibers of $0.5 \mathrm{~mm}$ diameter, $90 \mathrm{~mm}$ long and separated from its neighbors by $13 \mathrm{~mm}$. There are two x-y pairs of fiber beam monitors located $\sim 180^{\circ}$ and $\sim 270^{\circ}$ away from the injection point. The vertical-suspended fibers measure in x-direction and the horizontal-suspended fibers measure in y-direction. One thing that should be noticed is the fibers stay inside the vacuum and can be plunged into the beam path for measurement.

The Tracker can monitor the beam profiles in an indirect way by tracking the paths of decay positrons. This information will be used to study the systematic errors, as shown 

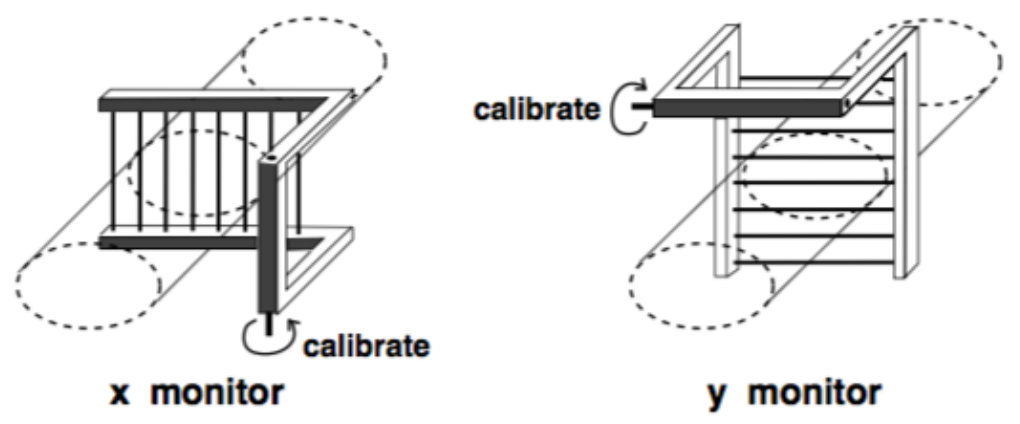

Figure 2.18: Schematic of Fiber Harp.

in Table 2.2. By measuring the radius of curvature of the decay positron path, one can find the point of the muon decay under the assumption that the decay positron is emitted tangentially relative to the equilibrium orbit on average. The decay point distribution is directly related to the stored muon distribution. The design is to have three tracker stations located at $\sim 15^{\circ}, \sim 180^{\circ}$ and $\sim 270^{\circ}$ from the injection point. Each tracker station consists of 8 tracker modules as shown in Fig. 2.19. The modules slot into the "staircase" walls of the modified vacuum chambers. A tracker module has four layers of straws arranged as two close-packed doublet planes in UV configuration oriented $\pm 7.5^{\circ}$ from the vertical direction, as shown in Fig. 2.20.

\begin{tabular}{llll}
\hline \hline Uncertainty & E821 value & E989 goal & Tracker's role \\
\hline Magnetic field seen by muons & $0.03 \mathrm{ppm}$ & $0.01 \mathrm{ppm}$ & $\begin{array}{l}\text { Measure beam profile on a fill by } \\
\text { fill basis ensuring proper muon } \\
\text { beam alignment }\end{array}$ \\
\hline Beam dynamics corrections & $0.05 \mathrm{ppm}$ & $0.03 \mathrm{ppm}$ & $\begin{array}{l}\text { Measure beam oscillation param- } \\
\text { eters as function of time in the fill }\end{array}$ \\
\hline Pileup correction & $0.08 \mathrm{ppm}$ & $0.04 \mathrm{ppm}$ & $\begin{array}{l}\text { Isolate time windows with more } \\
\text { than one positron hitting the } \\
\text { calorimeter to verify calorimeter } \\
\text { based pileup correction }\end{array}$ \\
\hline Calorimeter gain stability & $0.12 \mathrm{ppm}$ & $0.02 \mathrm{ppm}$ & $\begin{array}{l}\text { Measure positron momentum } \\
\text { with better resolution than the } \\
\text { calorimeter to verify calorimeter } \\
\text { based gain measurement }\end{array}$ \\
\hline Precession plane tilt & & & $\begin{array}{l}\text { Measure up-down asymmetry in } \\
\text { positron decay angle }\end{array}$ \\
\hline
\end{tabular}

Table 2.2: Systematic uncertainty goals related to Tracker for the Muon $g-2$ Experiment [12].

Calorimeters are used to measure the arrival time and energy of the decay positron. 


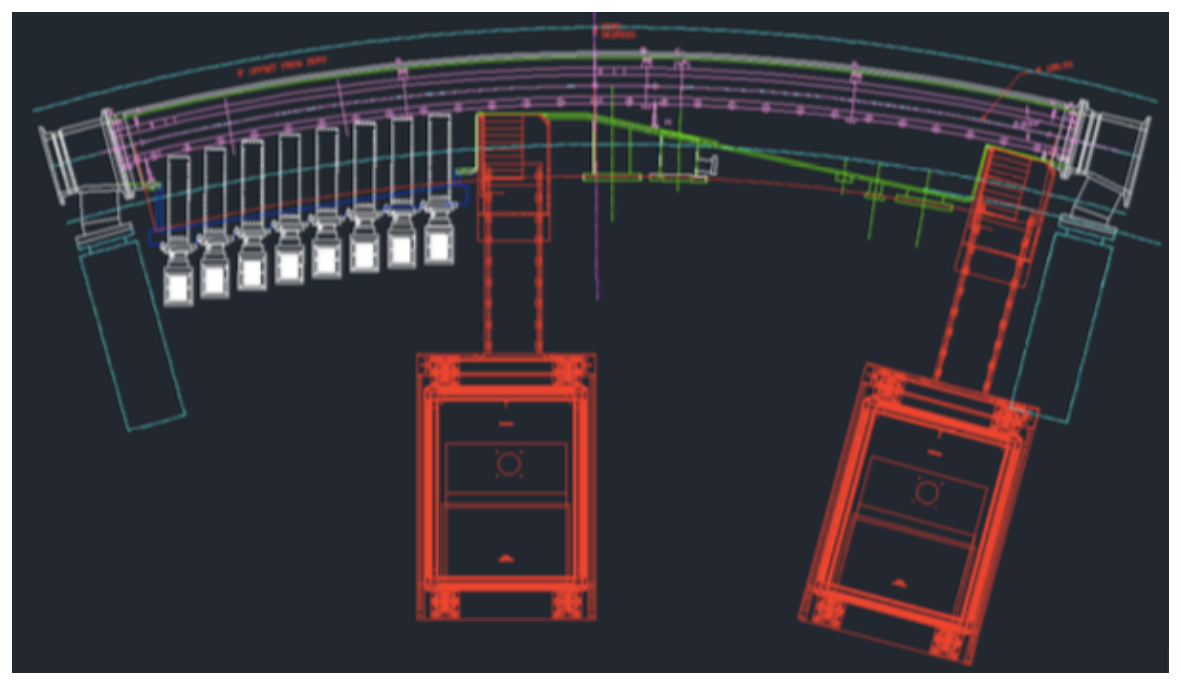

Figure 2.19: Placement of the straw tracker modules.

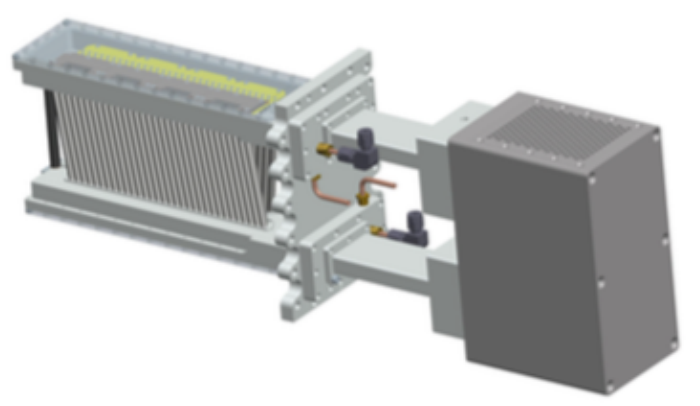

Figure 2.20: Schematic of a tracker module. 


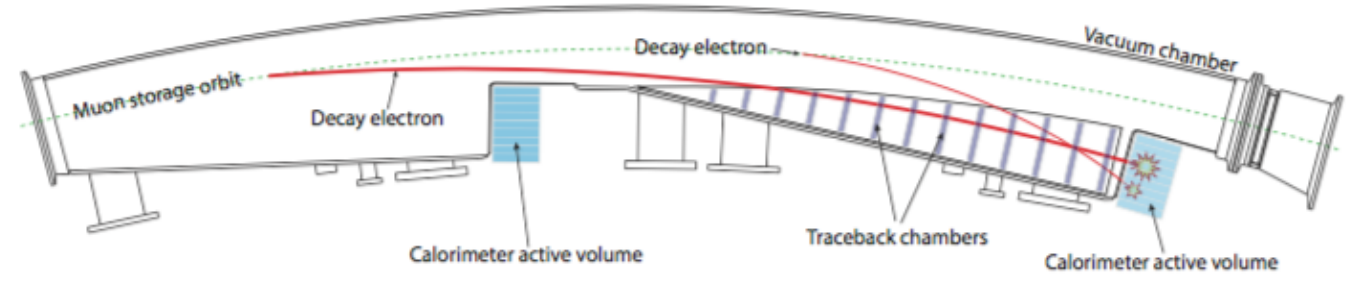

Figure 2.21: Schematic overview of decay positron hitting the calorimeter.

The average radial distribution of the stored muon beam can be determined by fast rotation analysis using the decay positron signals. The fast rotation analysis is based on time evolution of the muon bunch structure which can be modeled with the decay positron signals. Detailed discussion of fast rotation analysis will be presented in Chapter 5 .

\subsubsection{Detecting the Decay Positron Signals}

There are 24 electromagnetic calorimeters placed symmetrically around the inside of the ring to measure the energy and arrival time of decay positrons. Positrons from muon decay do not end up with sufficient momentum to fly along the muon equilibrium orbits. They will curl inward and hit the segmented lead fluoride calorimeters, as shown in Fig. 2.21.

Each of the E989 calorimeters consists of $54 \mathrm{PbF}_{2}$ Cherenkov crystals stacked in a 6 high by 9 wide array. An ultra-fast readout based on the latest generation of Hamamatsu MPPCs (SiPMs, or silicon photomultipliers) is used [66]. The calorimeter system and gain stability were tested at SLAC [67]. The experiment uses a $700 \mu$ s fill time. The times of all decay positrons from different fills must be properly aligned with respect to the time of injection, defined as $T 0$.

Experimentally, we apply an energy cut to select the decay positrons within a range of angles in the muon rest frame. Because of the parity violation, there exists a correlation between the muon spin and decay positron direction. This correlation allows the spin direction to be measured as a function of time. We then fill the decay positrons' arrival time into a histogram for our analysis of the anomalous spin precession frequency. The classic data display is shown in Fig. 2.22.

\subsection{Statistical and Systematic Errors}

The Fermilab Muon $g-2$ Experiment (E989) design features a four-fold improvement in the experimental precision compared with the BNL $g-2$ Experiment (E821) which reduces the error on measurement of $a_{\mu}$ to the $140 \mathrm{ppb}$ level. The statistical uncertainty of E989 is at the $100 \mathrm{ppb}$ level. To realize this, E989 must obtain twenty-one times the amount of data collected for E821. This means we need about $1.5 \times 10^{11}$ decay positron events in the final fitted histogram. The systematic errors of $a_{\mu}$ are derived from those on the anomalous spin precession frequency $\omega_{a}$ and the magnetic field normalized to the proton Larmor frequency 


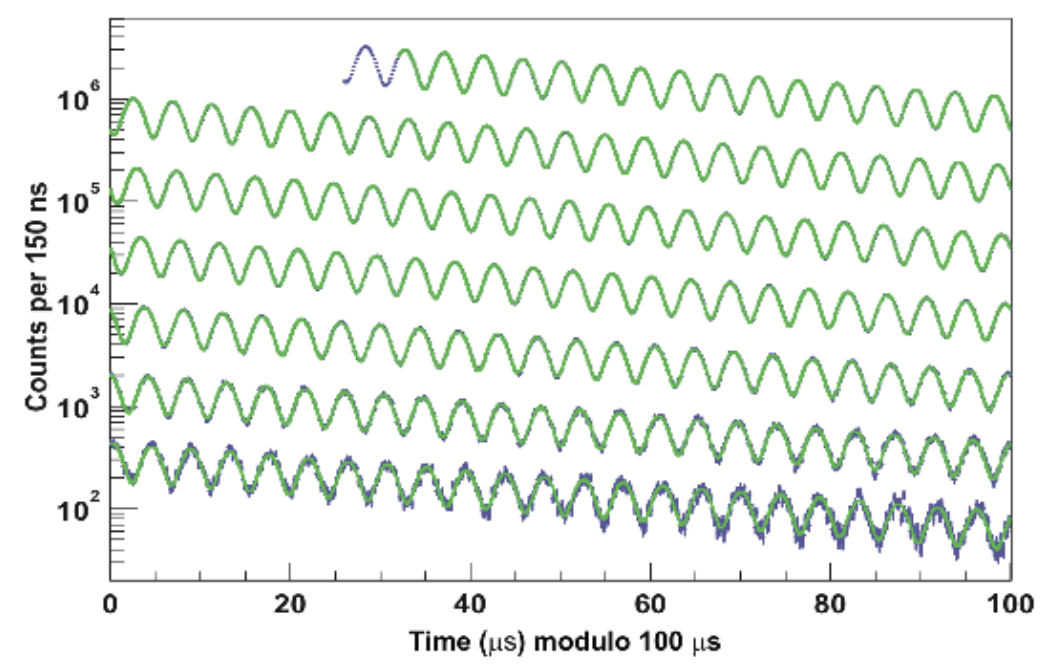

Figure 2.22: E821 decay positron arrival time histogram [44].

$\omega_{p}$, each of which is targeted to reach a $\pm 70 \mathrm{ppb}$ level. Compared to E821, there will be a three-fold improvement on $\omega_{a}$ uncertainties, and a two-fold improvement on $\omega_{p}$.

\subsubsection{Statistical Errors}

To determine $\omega_{a}$, we apply a least-squares fit or maximum likelihood fit to the histograms of decay positron events vs. time in the fill. For a good fit, the uncertainty $\delta \omega_{a}$ from the fits is purely statistical depending on the size of the data set used. The size of data set depends on the data accumulation rate and the running time, which are calculated from initial proton flux to fitted events in the final histograms. Many factors are related to the event-rate estimate, such as the proton fills per second, kinematics of the decay line length, kinematics of the decay line captures, storage ring kicker pulse shape, inflector, thinner or displaced Q1 outer plate, etc.

The E989 design estimates the need for a run duration of $17 \pm 5$ months, which includes 2 months of overall commissioning and 2 months of systematic studies. A sequential list of factors that affect the event-rate based on a bottom-up, full simulation approach is given in Table 2.3. For each $1.33 \mathrm{~s}$ supercycle with the Booster operating at $15 \mathrm{~Hz}$, we expect 4 batches of $4 \times 10^{12}$ protons delivered to the Recycler. Each proton batch will be split into four proton bunches of intensity $10^{12}$. Therefore, $g-2$ will receive 16 proton bunches per 1.33 s supercycle, which is equivalent to a rate of $12 \mathrm{~Hz}$. Each bunch refers to a "fill" of the storage ring (SR). Four sequential stages of the simulation are considered to estimate the positron record by detectors per fill: pion production on the target, muon capture from pion decay and subsequent transport to the storage ring entrance, muon transmission into and subsequent capture in the storage ring, and the decay positron acceptance by the detectors. 


\begin{tabular}{|l|l|l|}
\hline Item & Factor & Value per fill \\
\hline Protons on target & & $10^{12} \mathrm{p}$ \\
Positive pions captured in FODO, $\delta p / p= \pm 0.5 \%$ & $1.2 \times 10^{-4}$ & $1.2 \times 10^{8}$ \\
Muons captured and transmitted to SR, $\delta p / p= \pm 2 \%$ & $0.67 \%$ & $8.1 \times 10^{5}$ \\
Transmission efficiency after commissioning & $90 \%$ & $7.3 \times 10^{5}$ \\
Transimission and capture in SR & $(2.5 \pm 0.5) \%$ & $1.8 \times 10^{4}$ \\
Stored muons after scraping & $87 \%$ & $1.6 \times 10^{4}$ \\
Stored muons after $30 \mu \mathrm{S}$ & $63 \%$ & $1.0 \times 10^{4}$ \\
Accepted positrons above E=1.86 GeV & $10.7 \%$ & $1.1 \times 10^{3}$ \\
\hline \hline Fills to acquire 1.6 $\times 10^{11}$ events $(100 \mathrm{ppb})$ & & $1.5 \times 10^{8}$ \\
Days of good data accumulation & 17 hours $/ \mathrm{d}$ & $202 \mathrm{~d}$ \\
Beam-on commissioning days & & $150 \mathrm{~d}$ \\
Dedicated systematic studies days & & $50 \mathrm{~d}$ \\
\hline Approximation running time & & $402 \pm 80 \mathrm{~d}$ \\
\hline Approximation total proton on target request & & $(3.0 \pm 0.6) \times 10^{20}$ \\
\hline
\end{tabular}

Table 2.3: Event-rate calculation using a bottom-up approach [12].

\subsection{2 $\omega_{a}$ Systematic Errors}

The goal of the E989 is to keep the total combined systematic uncertainty of $\omega_{a}$ below 70 ppb. The systematic error studies include the hardware changes, data taking and analysis. The major sources come from the Coherent Betatron Oscillation (CBO), muon losses, pileup and gain, which can be broadly divided into two categories. The pileup and gain are corrections to the underlying data before a fit, while the CBO and muon losses are implemented in the function of fitting with floating parameters that allow the minimization to adjust as needed. A summary of $\omega_{a}$ systematic uncertainties based on the traditional $T$-method ${ }^{1}$ analysis of $\omega_{a}$ is given in Table 2.4 .

The gain systematic error is studied in a simulation and analysis where the gain perturbations could be applied. In general, this is to correct any systematic hardware gain drifts over the short term time scale of a fill, event by event for each calorimeter station and crystal. The gain correction function is prepared by detector response using both the known laser calibration pulses and the time stability of the pileup-corrected overall energy spectrum. The event-rate can change by more than four orders of magnitude during a $700 \mu$ s fill. Hence, a fill-scale gain perturbation of the form $G(t)=1+\epsilon \exp \left(-t /\left(\gamma \tau_{\mu}\right)\right)$ is used, where $\epsilon$ is the magnitude of the unknown perturbation. Here, $G(t)$ stands for the difference between the true gain vs. time behavior of the detector and electronics systems and the corrected one. The target gain stability for E989 is $\delta G / G<10^{-3}$ over a $700 \mu$ s fill. The simulation study of gain systematic error is given in Fig. 2.23, where we can see that after including the gain correction, the mean and width of the extracted $\omega_{a}$ distribution is restored to that of the

\footnotetext{
${ }^{1}$ In the experiment, the analysis based on the number of decay positrons with a energy threshold measured by calorimeters that changes periodically is called $T$-method; the analysis based on the energy of the decay positron measured by calorimeters that changes periodically is called $Q$-method.
} 


\begin{tabular}{|c|c|c|c|}
\hline Category & $\mathrm{E} 821[\mathrm{ppb}]$ & E989 Improvement Plans & Goal [ppb] \\
\hline \multirow[t]{2}{*}{ Gain changes } & 120 & Better laser calibration & \\
\hline & & low-energy threshold & 20 \\
\hline \multirow[t]{2}{*}{ Pileup } & 80 & Low-energy samples recorded & \\
\hline & & calorimeter segmentation & 40 \\
\hline \multirow{3}{*}{$\begin{array}{l}\text { Lost Muons } \\
\mathrm{CBO}\end{array}$} & 90 & Better collimation in the ring & 20 \\
\hline & 70 & Higher $n$ value (frequency) & \\
\hline & & Better match of beamline to ring & $<30$ \\
\hline \multirow[t]{2}{*}{$E$ and pitch } & 50 & Improved Tracker & \\
\hline & & Precise storage ring simulations & 30 \\
\hline Total & 180 & Quadrature sum & 70 \\
\hline
\end{tabular}

Table 2.4: Systematic errors estimated for the anomalous spin precession frequency $\left(\omega_{a}\right)$ measurement.
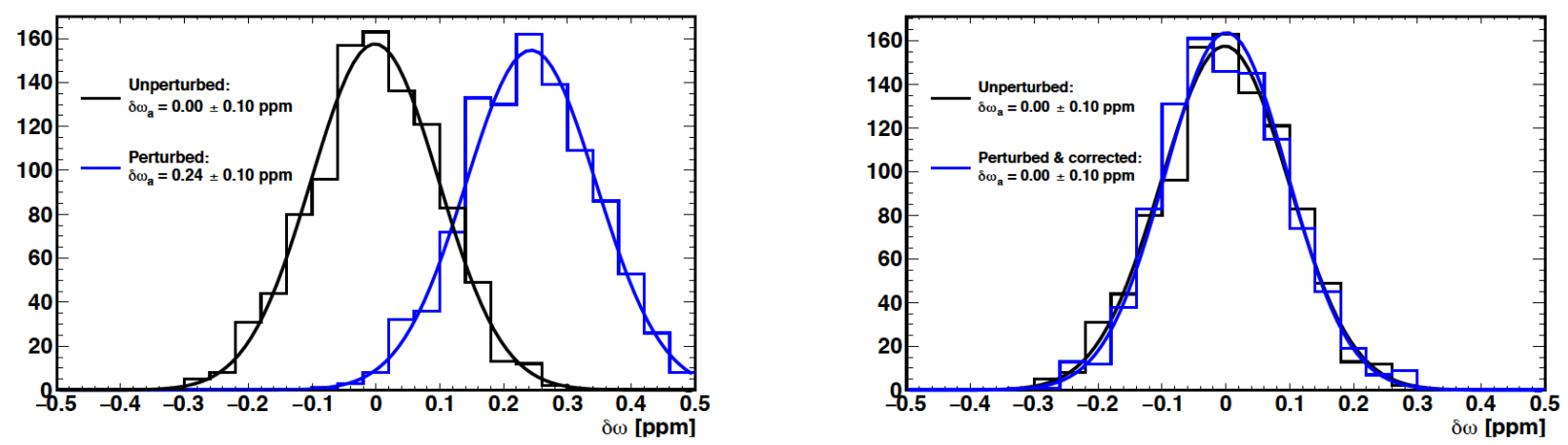

Figure 2.23: Histogram of extracted precession frequencies from 1000 simulated $T$ method histograms with and without an exponential gain perturbation of the form $5 \times$ $10^{-3} \exp \left(-t /\left(\gamma \tau_{\mu}\right)\right)$. The left plot does not include a correction from the simulated laser calibration while the right plot does.

unperturbed spectrum [12].

The term "Pileup" refers to the overlap of events in the calorimeter that originate from separate muon decays that are too close to each other in time and space to be resolved into individual pulses. In general, when two pulses overlap, the two individual events will be lost and one event with the sum of their energies is gained. There exists a finite time offset between two pulses and usually the recorded overlapped pulse shape is widened causing the combined amplitude to be somewhat less than the sum of two individual amplitudes. The fraction of pileup events increases with rate, and in addition, the muon spin precession phase varies with the energy of the pulse (higher energy usually refers a larger radius-orbit). This will cause an average early-to-late phase shift that directly distorts the fitting of $\omega_{a}$. Thus, the $(E, t)$ distribution of pileup pulses must be constructed and subtracted from the spectrum before we apply a fit. 
The CBO systematic error arises primarily from uncertainties in the frequency and functional form of the CBO. The muon loss systematic error occurs if the muons lost from the storage ring at late times have a different average spin direction compared to the stored muons. This happens due to the production and storage processes. Those beam dynamics related systematic errors will be discussed in Chapter 4.

\subsection{3 $\omega_{p}$ Systematic Errors}

The magnetic field is mapped by measuring the Lamar frequency of stationary protons with the NMR probes. The major sources of systematic errors arise from the calibration of the trolley probes, trolley measurements, fixed-probes, etc. A compact summary of the expected systematic uncertainties of E989 is given in Table 2.5 [12].

\begin{tabular}{|c|c|c|c|}
\hline Category & E821 [ppb] & E989 Improvement Plans & Goal [ppb] \\
\hline $\begin{array}{l}\text { Absolute field } \\
\text { calibration }\end{array}$ & 50 & $\begin{array}{l}\text { Special } 1.45 \text { T calibration magnet with } \\
\text { thermal enclosure; additional probes; } \\
\text { better electronics }\end{array}$ & 35 \\
\hline $\begin{array}{l}\text { Trolley probe } \\
\text { calibrations }\end{array}$ & 90 & $\begin{array}{l}\text { Plunging probes that can cross cali- } \\
\text { brate off-central probes; better position } \\
\text { accuracy by physical stops and/or op- } \\
\text { tical survey; more frequent calibrations }\end{array}$ & 30 \\
\hline $\begin{array}{l}\text { Trolley measure- } \\
\text { ments of } B_{0}\end{array}$ & 50 & $\begin{array}{l}\text { Reduced position uncertainty by factor } \\
\text { of } 2 \text {; improved rail irregularities; stabi- } \\
\text { lized magnet field during measurement }\end{array}$ & 30 \\
\hline $\begin{array}{l}\text { Fixed probe in- } \\
\text { terpolation }\end{array}$ & 70 & $\begin{array}{l}\text { Better temperature stability of the } \\
\text { magnet; more frequent trolley runs }\end{array}$ & 30 \\
\hline $\begin{array}{l}\text { Muon distribu- } \\
\text { tion }\end{array}$ & 30 & $\begin{array}{l}\text { Additional probes at larger radii; } \\
\text { improved field uniformity; improved } \\
\text { muon tracking }\end{array}$ & 10 \\
\hline $\begin{array}{l}\text { Time-dependent } \\
\text { external mag- } \\
\text { netic fields }\end{array}$ & - & $\begin{array}{l}\text { Direct measurement of external fields; } \\
\text { simulations of impact; active feedback }\end{array}$ & 5 \\
\hline Others & 100 & $\begin{array}{l}\text { Improved trolley power supply; trol- } \\
\text { ley probes extended to larger radii; re- } \\
\text { duced temperature effects on trolley; } \\
\text { measure kicker field transients }\end{array}$ & 30 \\
\hline $\begin{array}{l}\text { Total systematic } \\
\text { error on } \omega_{p}\end{array}$ & 170 & Quadrature sum & 70 \\
\hline
\end{tabular}

Table 2.5: Systematic errors estimated for the magnetic field $\left(\omega_{p}\right)$ measurement. 


\section{CHAPTER 3}

\section{ELECTROSTATIC QUADRUPOLE SYSTEM}

The Electrostatic Focusing Quadrupoles (ESQ or QUAD) are introduced to confine muons vertically in the storage ring. The ESQ were first used for beam storage in the CERN III $g-2$ experiment [48]. E989 reuses the ESQ plates built at BNL [44], but with some careful refurbishment and necessary upgrades as will be discussed in the following sections. Ideally, we would want the ESQ to cover the full storage ring. But we have to leave spaces for the other subsystems, such as Inflector, Kickers, Fiber Harps, Straw Trackers, etc. Therefore, we have a lattice design of the ESQ with a four-fold symmetry inside the storage ring, as shown in Fig. 2.14. Each ESQ region contains a short quadrupole electrode length of $1.6 \mathrm{~m}$ and a long quadrupole electrode length of $3.2 \mathrm{~m}$. The total quadrupole electrodes occupy $43 \%$ of the total circumference of the storage ring. The four-fold symmetry keeps the variation in the beta function small, $\sqrt{\beta_{\max } / \beta_{\min }}=1.04$, which minimizes beam "breathing" and improves the muon orbit stability. Each quad segment consists of a short quad of $13^{\circ}$ and a long quad of $26^{\circ}$ for two reasons: 1) to make every quadrupole chamber independent of others, facilitating their development, testing, etc., and 2) to reduce the extent of low energy electron trapping [68].

The ESQ produce the vertically focusing electrostatic quadrupole field for the muon beam. There are some new requirements for the E989 ESQ. Our vacuum condition is about $10^{-6}$ Torr or better, and the ESQ should have stable operation during extended periods of time in such a vacuum environment. E989 has a wide injection beam pulse, and the ESQ should have reliable operation in pulsed mode with the beam time structure. The operating point should be in a resonance-free region at higher $n$ value, i.e., $n=0.185$, primarily to change the horizontal coherent betatron oscillations (CBO) frequency away from near twice the muon $g-2$ frequency, as well as to store more muons with a high event-rate. At the places where trajectories of incoming muons and decay positrons intercept parts of the ESQ (Q1 outer plates), the ESQ should have the minimum possible amount of material to optimize the muon storage efficiency. E989 also requires ESQ quality and stability sufficient to keep the beam dynamics systematic uncertainties well below the measurement goal.

In the following sections, the ESQ (quad) plate alignment, the quad extension and readout system will be discussed.

\subsection{Quad Plate Alignment}

In the Fermilab Muon $g-2$ Experiment, four Electrostatic Focusing Quadrupoles (ESQ) will provide the vertical focusing of the muon beam. Errors in the electric field of the quadrupoles will give rise to periodic forces which perturb the muon orbits as shown in 


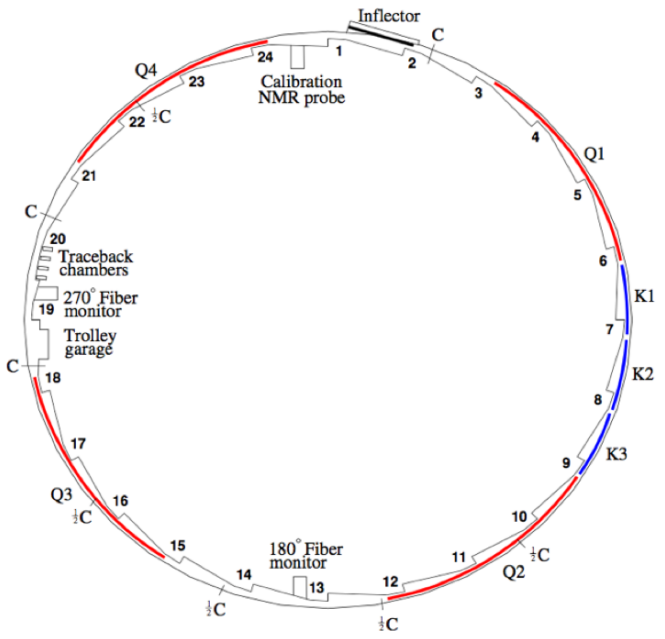

Figure 3.1: Schematic view of muon storage ring: ESQ regions are marked as Q1-Q4.

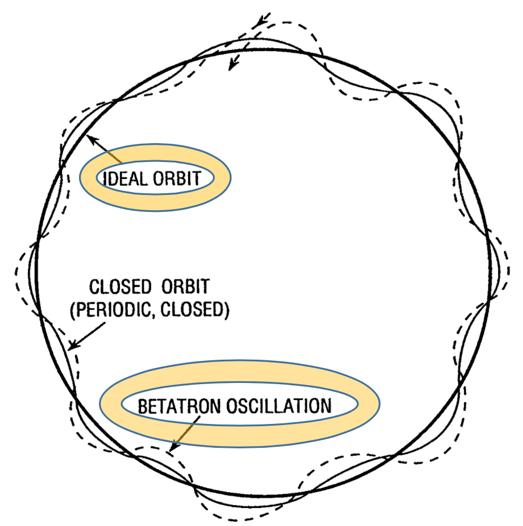

Figure 3.2: Diagram illustrating the ideal orbit, the closed orbit due to non-ideal fields, and betatron oscillations about the closed orbit [70].

Fig. 3.2. If the periodicity of the force falls on some resonances, the horizontal or vertical oscillations may increase, leading to a loss of muons [69].

The $g-2$ storage ring acts as a weak-focusing betatron, with the vertical focusing provided by electrostatic quadrupoles. A pure quadrupole electric field provides a linear restoring force in the vertical direction, and the combination of the (defocusing) electric field and the central (dipole) magnetic field $\left(B_{0}\right)$ provides a net linear restoring force in the radial direction. If the ring is operated at the magic momentum, the electric field does not contribute to the spin precession, since the second term in Eq. (3.1) will vanish (with the assumption that $\vec{\beta} \cdot \vec{B}=0$ and $\left.\gamma=\gamma_{\text {magic }} \approx 29.3\right)$. However, the radial electric field will give a second-order correction to the spin frequency. To the extent that $\vec{\beta} \cdot \vec{B} \neq 0$, there is also a correction from the vertical betatron motion. Therefore, to decrease errors in the electric field of the quadrupoles, careful quad plate alignment is necessary and important to obtain a high precision measurement of 


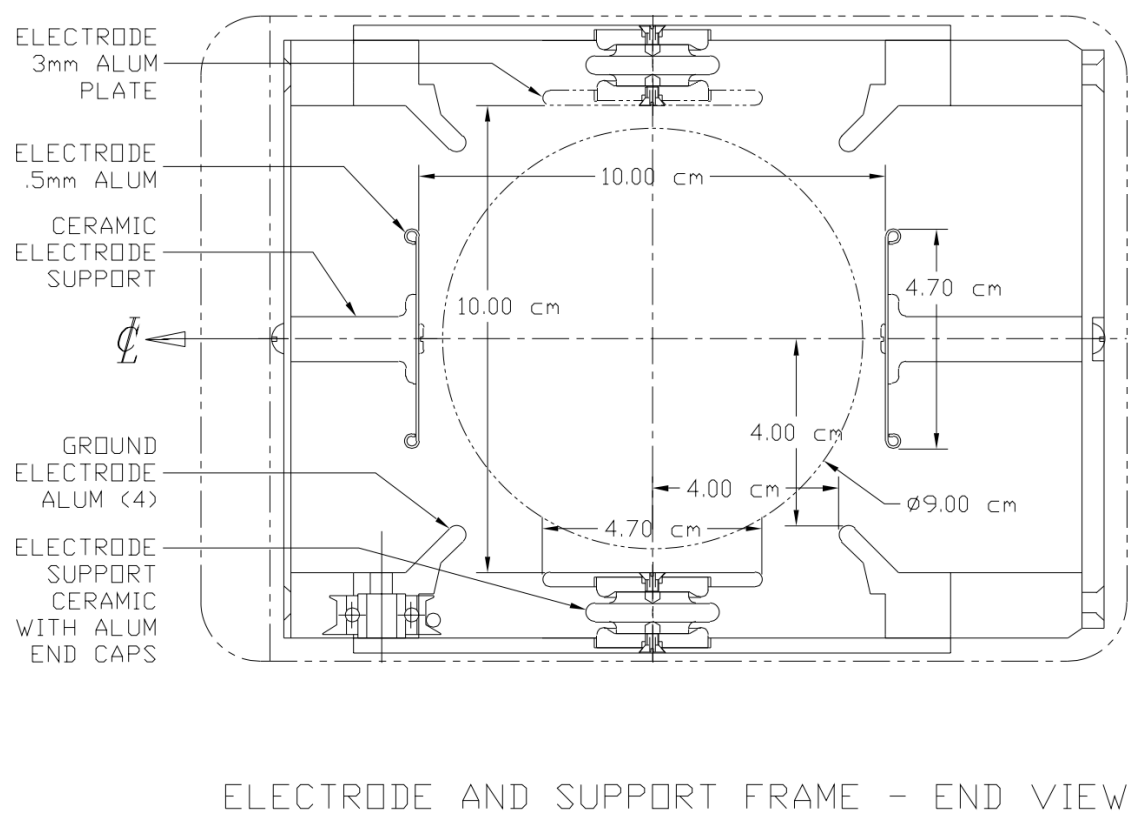

Figure 3.3: A schematic of the quadrupole cross-section.

$a_{\mu}$.

$$
\vec{\omega}_{a}=-\frac{Q e}{m}\left[a_{\mu} \vec{B}-\left(a_{\mu}-\frac{1}{\gamma^{2}-1}\right) \frac{\vec{\beta} \times \vec{E}}{c}\right]
$$

\subsubsection{Alignment Requirements}

A schematic representation of a cross-section of the electrostatic quadrupoles is shown in Fig. 3.3 with the various dimensions indicated. Four flat aluminum plates ("electrodes") are symmetrically placed around the $90-\mathrm{mm}$-diameter muon storage region. The placement accuracy requirements of ESQ plates in E821 were $\pm 0.5 \mathrm{~mm}$ for the vertical (lower/upper) quad electrodes and $\pm 0.75 \mathrm{~mm}$ for the horizontal (side) quad electrodes [68]. For the Fermilab Muon $g-2$ Experiment, we maintain those requirements and state that the standard deviation (RMS) over a short quad length should be less than $\pm 0.5 \mathrm{~mm}$ for the vertical quad plates and $\pm 0.75 \mathrm{~mm}$ for the horizontal quad plates. As for an individual point, the deviation can be as large as $\pm 2 \mathrm{~mm}$, which will keep the quad plates away from the trolley's trajectory [71]. In fact, many quad plates are aligned better than these requirements as shown in Appendix $A^{1}$.

The goodness of the alignment can be estimated by its effects on the electric field as well as the beam orbits. The quadrupoles' electric field can by analyzed with OPERA-2D and/or OPERA-3D [72]. The muon orbital motion is described by the beam dynamics formalism

\footnotetext{
${ }^{1}$ For the case of Q1 Outer plates, the real alignment is also much better than that shown in Appendix A. The later only reflects the deviation of middle part, which is normally worse than the average.
} 
[70]. The electric field correction to $a_{\mu}$ can be evaluated with the so-called fast rotation analysis, which will be discussed in Chapter 5 .

The absolute ESQ alignment should contain three parts: 1) quad plate alignment relative to the cage, 2) cage alignment relative to the vacuum chamber, and 3) vacuum chamber alignment relative to the storage ring. In this thesis, we only focus on the quad plate alignment part. For each quadrupole, the alignment sequence for the four electrodes is not so strict. However, it is convenient to install the vertical plates (lower/upper) and align them first. Moreover, this will make our final check with the laser alignment system easily.

\subsubsection{Investigation of Quad Plate Alignment with Capacitec}

Our initial quad plate survey was done with the Capacitec tool. Capacitec is a global technology company dedicated to advancing the capacitive principle of measurement physics to its highest level in sensor design. A realized claim of extremely high sensitivity to minute physical/mechanical changes and exceptional amplifier output stability support capacitive sensor applications especially in severe environments of temperature, magnetic fields, high radiation and non-contact, non-intrusive applications [73]. For the Capacitec measurement, basically there are two steps: recalibration and measurement.

\section{Recalibration of the Capacitec:}

There are two main reasons that we need to execute recalibration of the Capacitec amplifiers. First, we need to set a linear range to meet our measurement requirements (around $0 \sim 3 \mathrm{~mm})^{2}$; second, we need to investigate how well the linear relation of the distance vs. voltage is such that we can eventually use this to determine the distance between the sensor and the plate by reading the voltage.

Capacitec non-contact displacement probes are noted for their large linear range versus the small outside diameter of the probe package. The total range of measurable displacement is proportional to the probe size - the larger the probe outside diameter, the greater the gap. Typically, the maximum range achievable with any probe is the diameter of the sensor [73]. Our probes have a diameter around $8 \mathrm{~mm}$, however, for reasons such as malfunction of the readout, damage of the sensors or cable noise, we can only achieve a linear range around $0 \sim 3 \mathrm{~mm}$. Nevertheless, this is enough for our measurements.

The Capacitec we use for quad plate survey and the calibration setting for recalibration are shown in Fig. 3.4. A 30-min warmup is needed for best accuracy. The Amplifier series is model 4100-SL, and the slots used are 3 and 4 . Slot 3 is connected with one of the two sensors, which we call sensor CH1; slot 4 is connected with the other one called sensor CH2. The readouts we used are shown in Fig. 3.5. One of the readouts, KEITHLEY 197, was unstable, which turned out to be a malfunction, i.e., even without any input, the output was keeping changes from 0 to around 0.5 volts. Regardless, it still told us the rough linear range. We replaced it with another stable readout MAS 343 afterwards.

\footnotetext{
${ }^{2}$ Here, the required distortion of the quad plate is less than $1 \mathrm{~mm}$, the range of around $0 \sim 3 \mathrm{~mm}$ can allow us to move the trolley inside the cage without problems as well as tolerate the misalignment of the trolley rails.
} 

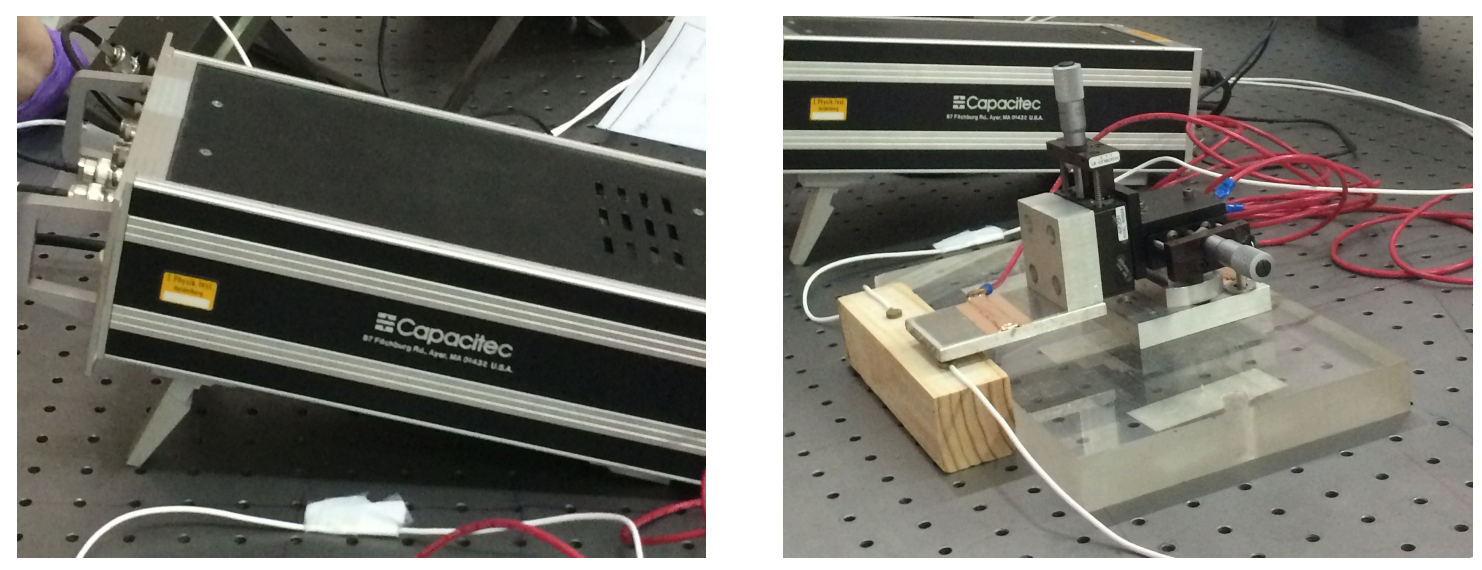

Figure 3.4: The Capacitec used for quad plate survey (left) and the calibration setting for recalibration (right).
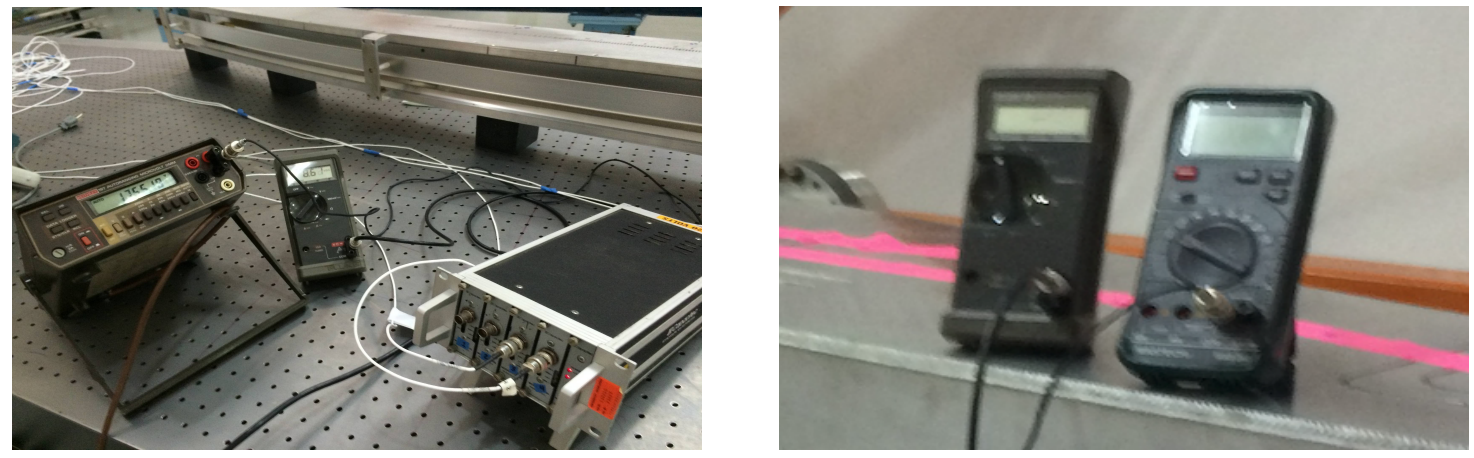

Figure 3.5: The readouts of the Capacitec. 


\begin{tabular}{|c|c|c|c|c|c|c|}
\hline Adjustment & OFFSET & GAIN & BREAK 1, LIN 1 & BREAK 1, LIN 1 & BREAK2 & BREAK 2 \\
\hline Position $(\mathrm{mm})$ & 0 & 0.5 & 1 & 1.5 & 2 & 2.5 \\
\hline Readout $(\mathrm{V})$ & 0 & 2.28 & 4.56 & 6.84 & 9.12 & $11.12^{3}$ \\
\hline
\end{tabular}

Table 3.1: Procedures for recalibration of Sensor CH1.
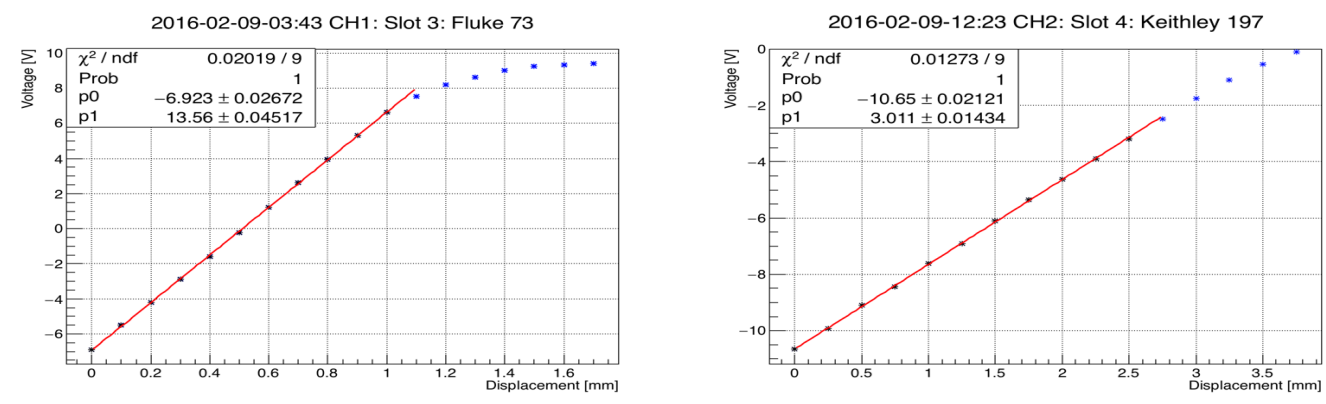

Figure 3.6: Capacitec probes before recalibration.

After several tests, we found out that the sensor $\mathrm{CH} 2$ already had a linear range of around $0 \sim 3 \mathrm{~mm}$; while sensor CH1 only had a linear range of around $0 \sim 1 \mathrm{~mm}$. These are shown in Fig. 3.6. The linear range of sensor $\mathrm{CH} 2$ not only meets our measurement requirement, but also looks very good since the value of the chi-square is very small and the probability is about 1 as shown in Fig. 3.6. Though the readout starts at a negative value, which could be adjusted to zero or a positive value, it is not a problem for our measurements and we just left the amplifier corresponding to $\mathrm{CH} 2$ as it was.

To achieve the required range for sensor CH1, we needed to do recalibration following the generic probe recalibration instructions. The control positions of the Capacitec amplifiers we use are not the same as the instructions describe. Specially, we do not have the Capacitec calibration stand. However, the idea of the recalibration is straightforward. We use a calibration setting shown in Fig. 3.4 instead. There are some front panel adjustable potentiometers, such as DRIVE, GAIN, OFFSET, BREAK 1, LIN 1, BREAK 2 and LIN 2, which can be used for recalibration. In Table3.1, we list our procedures for recalibration of sensor CH1. The result after recalibration of sensor $\mathrm{CH} 1$ is shown in Fig. 3.7, from which we can tell there is a good linear range of $0 \sim 2.5 \mathrm{~mm}$.

People may ask questions like "How good is the Capacitec calibration?" and "Can we trust the calibration?". To answer such questions, we repeat the calibration many times. The linear range for the probes is good, but we also want to know the systematic uncertainties of the calibration which will eventually affect our quad plate measurement.

We assume that the linear relation here is given by

$$
y=m x+c
$$

where $m$ is the slope and $c$ is the intercept. For a set data of points, ROOT Fit can easily give us these two parameters with their uncertainties $(m=p 1$ and $c=p 0$ for a linear fit

\footnotetext{
${ }^{3}$ Here, it is hard to get a 11.4 .
} 


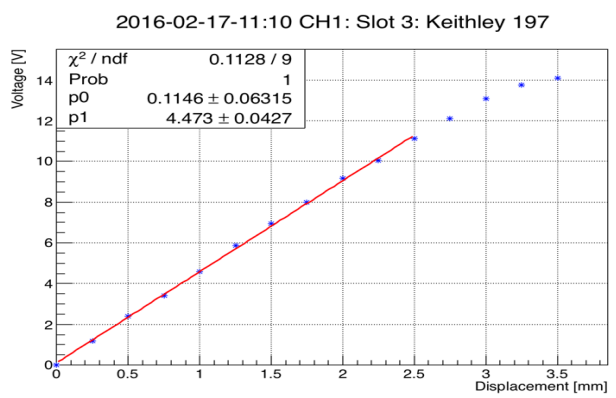

Figure 3.7: Recalibration of Sensor CH1.

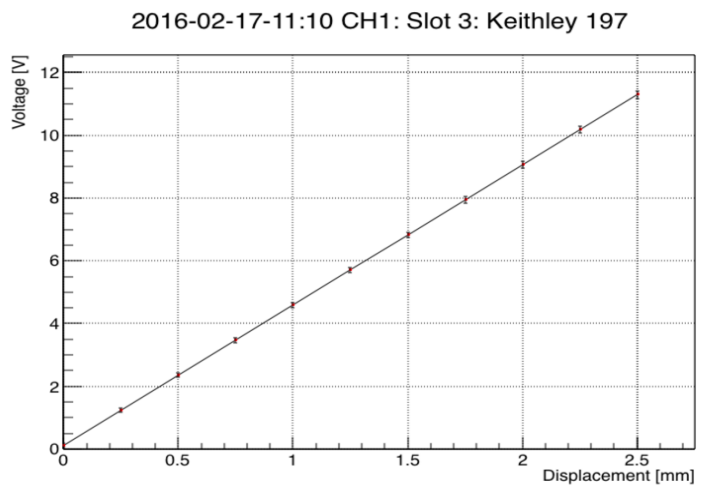

Figure 3.8: $y_{i}$ uncertainties of data shown in Fig. 3.7. 


\begin{tabular}{|c|c|c|c|c|c|c|c|c|c|c|c|}
\hline$x_{i}$ & 0 & 0.25 & 0.50 & 0.75 & 1.00 & 1.25 & 1.50 & 1.75 & 2.00 & 2.25 & 2.50 \\
\hline$y_{i}$ & 0.115 & 1.233 & 2.352 & 3.470 & 4.588 & 5.706 & 6.825 & 7.943 & 9.061 & 10.179 & 11.298 \\
\hline$\sigma_{y_{i}}$ & 0.063 & 0.064 & 0.067 & 0.071 & 0.076 & 0.083 & 0.090 & 0.098 & 0.106 & 0.115 & 0.124 \\
\hline
\end{tabular}

Table 3.2: $y_{i}$ uncertainties of recalibration part in Fig. 3.7.

"pol1"). We are interested in how the quantity $y$ changes under variations in $m$ and $c$. The uncertainty $\sigma_{y}$ is given by [74]

$$
\sigma_{y}^{2} \approx x^{2} \sigma_{m}^{2}+\sigma_{c}^{2}
$$

where we have ignored the high order terms and the correlated terms.

To understand Eq. (3.3), we declare that the $y_{i}$ measurements are not correlated and the $x_{i}$ values are assumed to be known with no or negligible uncertainty. In this case, it will be clear that the uncertainty of $y$ is related to the physical quantity $x$. For example, the uncertainty $\sigma_{y}$ of data shown in Fig. 3.7 can be calculated with Eq. (3.3), the results are given in Fig. 3.8 and Table 3.2. From Table 3.2, we can see the uncertainties are less than $1.25 \%$, which agree with the Capacitec amplifiers' linear performance $\pm 0.2 \% \sim \pm 2 \%$ [73].

However, some possible error sources may be introduced for our Capacitec recalibration as shown in Fig. 3.9. The "close effect" here means that the distance of the gap between the sensor surface and the plate surface during the calibration procedure is too small. Since we do not have the calibration stand, we can only try to make the gap as small as possible. The question arises how we can tell whether it is small enough. Here, the gap between the sensor surface and the baseline (the closest position of the plate surface to sensor) does affect our measurements. This seems inevitable since we are using non-contact probes. For our calibration, we try to make this gap around or less than $0.25 \mathrm{~mm}$. Consequently our measurement should have a positive correction.

We need to consider this "close effect" if we want to determine the distance correctly. Is this a problem? For our plate measurement, i.e., top/bottom plates, we want to get a distortion less than $\pm 0.5 \mathrm{~mm}$. This "close effect" just causes a correction to the measurement results (the position of the plates) and will not affect the result of distortion. Once we consider this effect, or we have other ways to determine the position of the plates (some special positions would be enough) and compare this effect with their results, we would have great confidence to get rid of this "close effect". One possible way to test this effect is to use the Capacitec to measure some distances we know explicitly. By comparing the results from Capacitec measurement with the answers, we will be able to sort out the correction due to the "close effect".

The "edge effect" shown in Fig. 3.9, indicates recalibration with the sensor beneath the corners (i.e., the right front corner) of the plate. Comparing the calibration with the sensor beneath the middle of the plate, this "edge effect" will cause some differences in our measurements. We want to know how bad this effect is and if we can avoid it. The answer to the latter is yes if we consider the different situations and treat them separately. For example, if we want to measure the distance between the sensor and the middle of each plate, we can just use the calibration data with the sensor beneath the middle of the plate; if we want to 

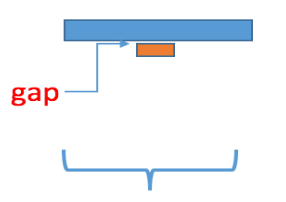

"close effect" (view from profile)

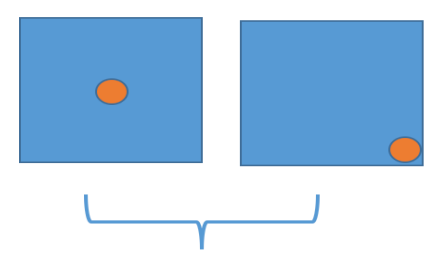

"edge effect" (view from top)

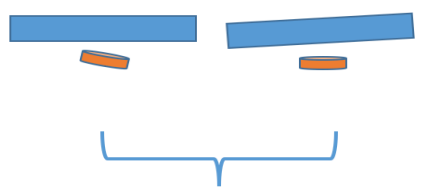

"angle effect"

(view from profile)

Figure 3.9: Three effects of Capacitec calibration.
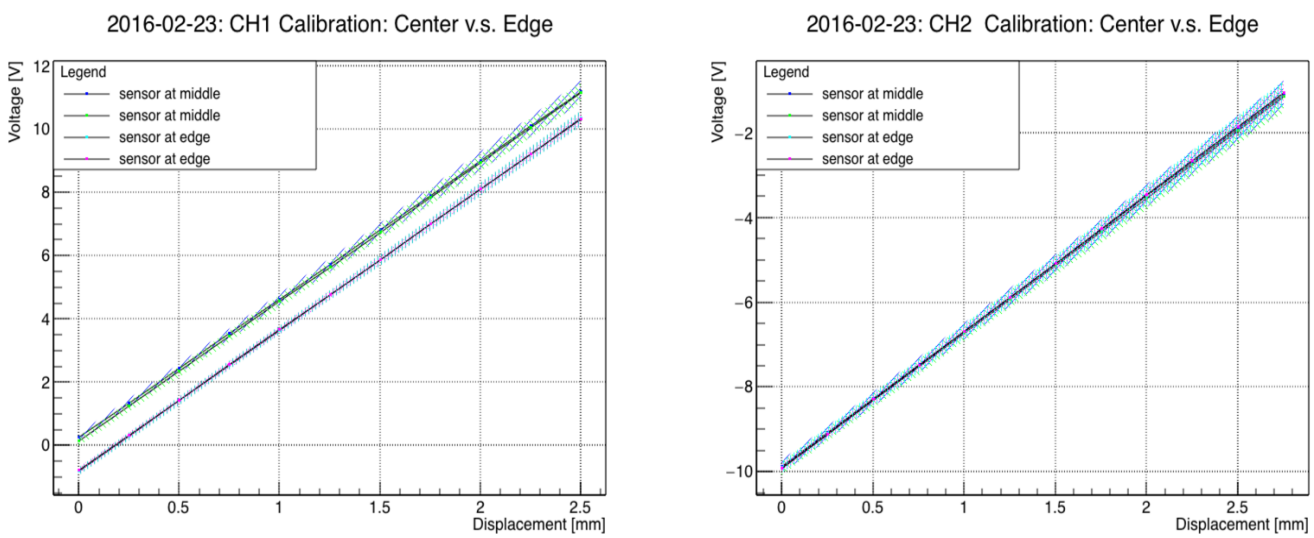

Figure 3.10: Calibration: sensor at the center of the plate versus sensor at the edge of the plate, with $3 \sigma_{y_{i}}$.

measure the distance between the sensor and the edges of each plate (to check whether the two horizontal/vertical plates are parallel to each other), we can use the calibration data with the sensor beneath the edge/corner of the plate. Applying these, we certainly can avoid the so called "edge effect".

However, it would be very interesting to know the uncertainties due to the "edge effect" or how bad this effect is. For the parallel-plate model of a capacitor, we know that the linear relation of distance vs. voltage based on the assumption that the length and width of the plates (the surface of the sensors and calibration plates) are much greater than their separation distance (the distance we measure). The results of calibration with the sensor at the middle of the plate and the sensor at the edge of the plate are given in Fig. 3.10, where we have considered $3 \sigma_{y_{i}}$ in order to distinguish different plots. Note that there is an obvious gap between the plots with the sensor at middle and plots with the sensor at edge for CH1 in the left graph in Fig. 3.10, while there is no obvious gap for that of $\mathrm{CH} 2$ in the right graph in Fig. 3.10. There are two possible reasons for this: when we perform the recalibration, it is very hard to make sure we have the same baseline, which is called the "close effect" discussed in the previous section; for the same baseline, there does exist an "edge effect" we have to deal with.

However, since the uncertainty of $y_{i}$ becomes large as $x_{i}$ increases, we assert that we 

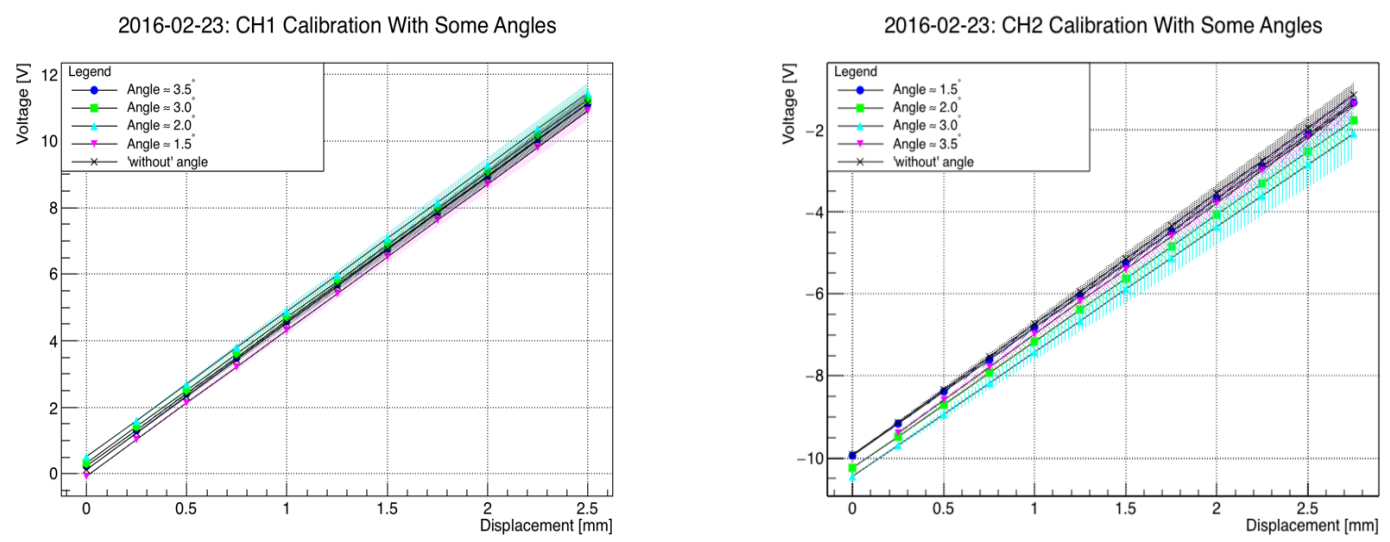

Figure 3.11: Calibration with some angle between the sensor surface and the plate surface, with $3 \sigma_{y_{i}}$.

only care about the change of the slope due to the "edge effect" here. In fact, the baseline problem can be adjusted by the methods discussed in the previous section. Moreover, if we are only concerned about the distortion of the plates, this would not be a problem at all. For the gap mentioned above, we have the same conclusion. From Fig. 3.10, we can see that due to the "edge effect", the slope is changed by around $+0.07 \mathrm{~V} / \mathrm{mm}$ for $\mathrm{CH} 1$ and +0.035 $\mathrm{V} / \mathrm{mm}$ for $\mathrm{CH} 2$. The largest uncertainty of the slope for $\mathrm{CH} 1$ would be around $2.35 \%$ and that of $\mathrm{CH} 2$ would be around $2.25 \%$. If we try to make sure the distance between the sensor and the plate surface is small, the uncertainties of $y_{i}$ will decrease significantly (from Eq. $(3.3))$.

The "angle effect" means the corrections of calibration due to some angles between the sensor surface and the plate surface, as shown in Fig. 3.9. Due to the distortions of the cage, rails or plates, or non-ideal placements of the sensor, some angle between the plate and sensor will exist unavoidably. We want to know how this "angle effect" will affect our measurement and how bad it is. If there is some angle $\theta$ between the sensor surface and the plate surface, the projected area of the sensor onto the plate will become $A \cos \theta$ ( $A$ is the area of the sensor surface). Assume the average distance between the sensor and plate can be represented by the distance from the center of the sensor surface to the plate, the correction due to this should be given by $-(1-\cos \theta)$. For an angle $\theta=5^{\circ}$, this gives a correction of about $-0.38 \%$.

To test the "angle effect", we try to make some angles by adding some spacers in one side of the wood box shown in Fig. 3.4. The angles can be sorted out by using trigonometry. Since the calibration settings we use are not perfect, we cannot determine the angle exactly. Nevertheless, we try to test the possible uncertainties. Some test results are shown in Fig. 3.11 , where we have considered $3 \sigma_{y_{i}}$ in order to distinguish different plots, as we deal with Fig. 3.10.

As a summary of the Capacitec recalibration, we determine that the Capacitec tool we have may not be good enough to keep the quad plate alignment well below the required tolerances. We consider the micrometer tools instead. 

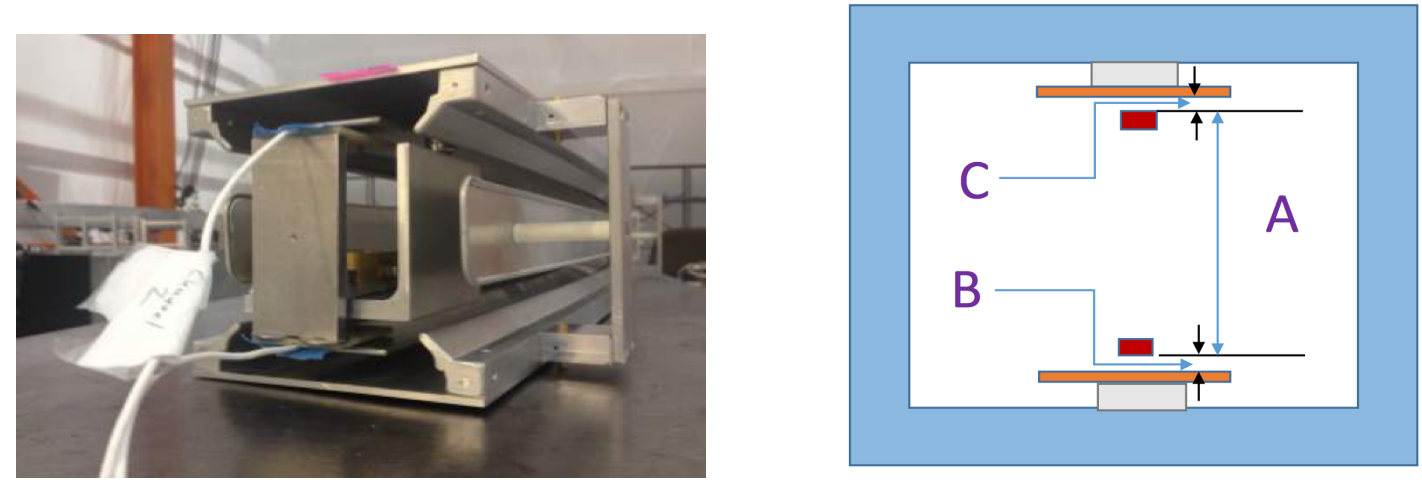

Figure 3.12: The scheme of Capacitec Measurement: A stands for the distance between two sensors; B stands for the distance between bottom (inner) sensor and bottom (inner) plate; $\mathrm{C}$ stands for the distance between top (outer) sensor and top (outer) plate. The distance between two plates equals $A+B+C$.

\section{Capacitec Measurement:}

The Capacitec measurement uses the results of recalibration to convert the voltage to distance. Before we move to the quad plate measurements, we want to discuss the uncertainties of the Capacitec measurement. From Eq. (3.2), we get

$$
x=\frac{y-c}{m}
$$

The uncertainties to the distance can be estimated using the following equation

$$
\sigma_{x}^{2} \approx\left(\frac{1}{m}\right)^{2} \sigma_{y}^{2}+\left(-\frac{y-c}{m^{2}}\right)^{2} \sigma_{m}^{2}+\left(-\frac{1}{m}\right)^{2} \sigma_{c}^{2}
$$

where we disregard the higher order terms and assume that the correlation terms are not important here. As an example, for the above situation of $\mathrm{CH} 1$, the largest variance we can have is about $\pm 0.20 \mathrm{~mm}$; while for $\mathrm{CH} 2$, it is about $\pm 0.50 \mathrm{~mm}$.

The scheme of using Capacitec to measure the distance in the quad plate alignment can be shown in Fig. 3.12. Some test results are shown in Fig. 3.13, where we list two data sets.

\subsubsection{Alignment with Micrometer Tools}

From the quad plate survey with the Capacitec, we understand the plate deviation situation and recognize the challenges to completing the plate alignments. The Capacitec was not

sufficiently stable and reliable to achieve our alignment. Our final alignment was achieved by using the micrometer tools designed by J. Grange and H. Nguyen, as shown in Fig. 3.14.

To adjust the plate position, we consider adding either a half spacer (washer) or a whole 

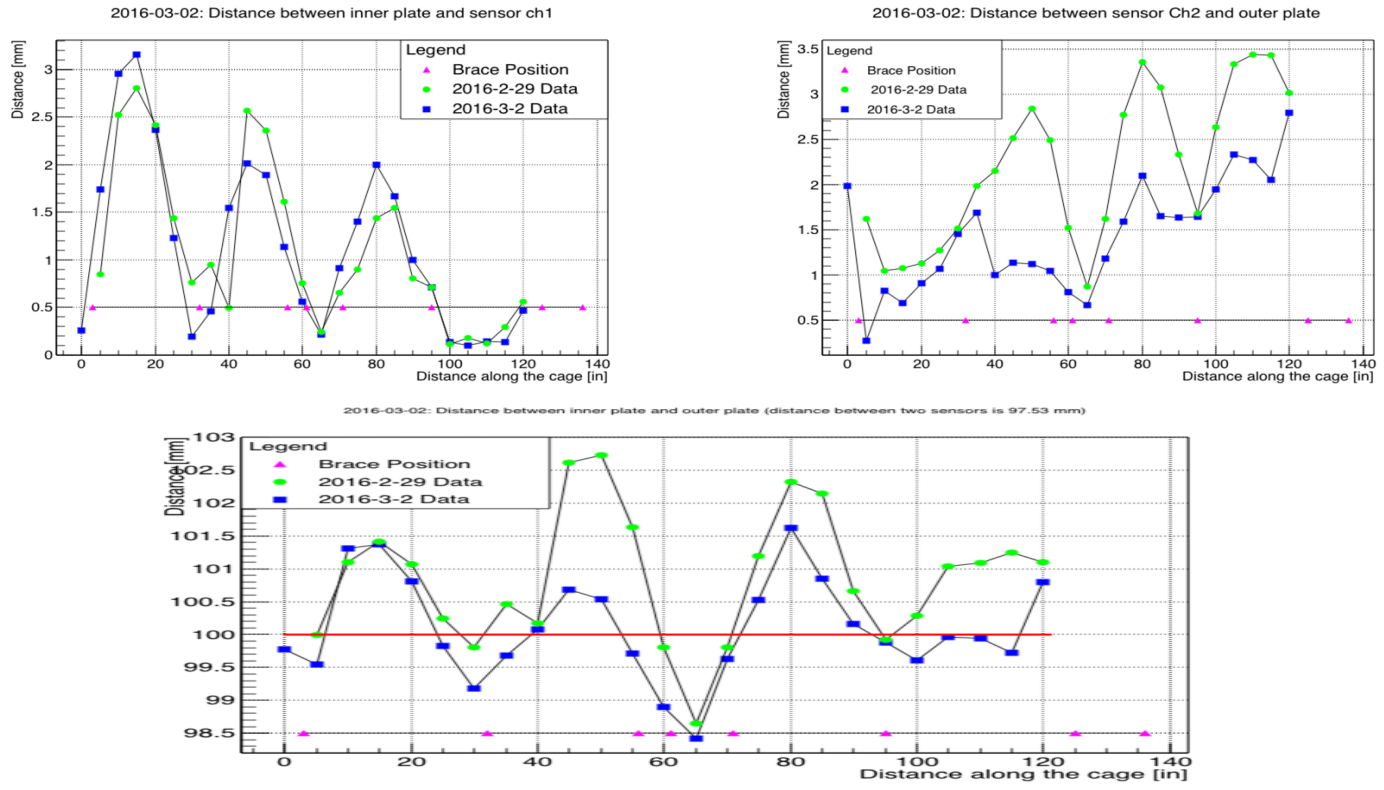

Figure 3.13: Quad plate alignment test with Capacitec tool: Blue data is after we perform adjustments, e.g.., adding spacers to the standoff support; Green data is before we perform these adjustments.
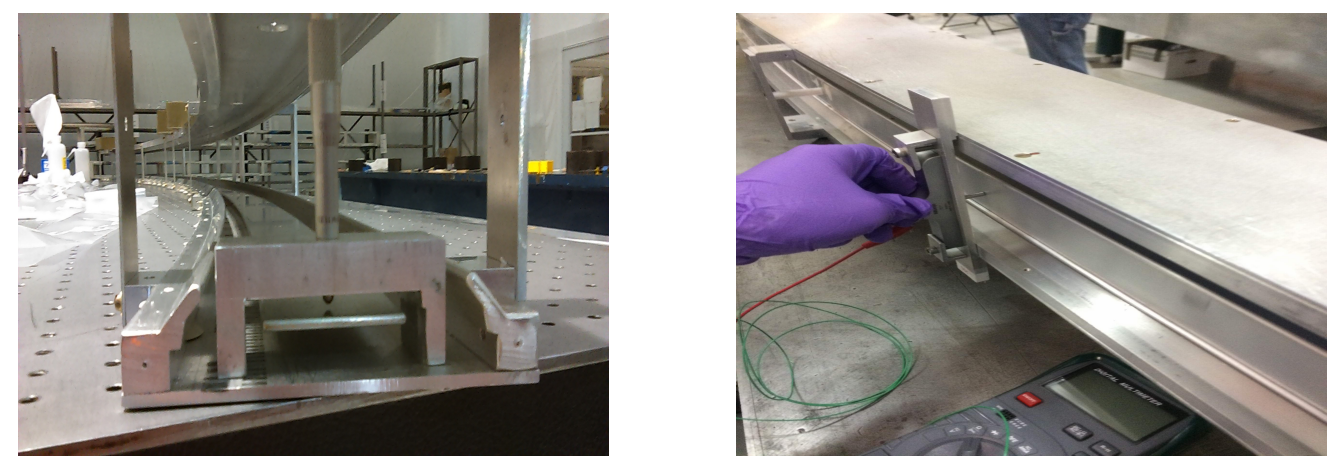

Figure 3.14: Micrometer tools for quad plate alignment: vertical tool (left) for upper/lower plates; radius tool (right) for inner/outer plates. 


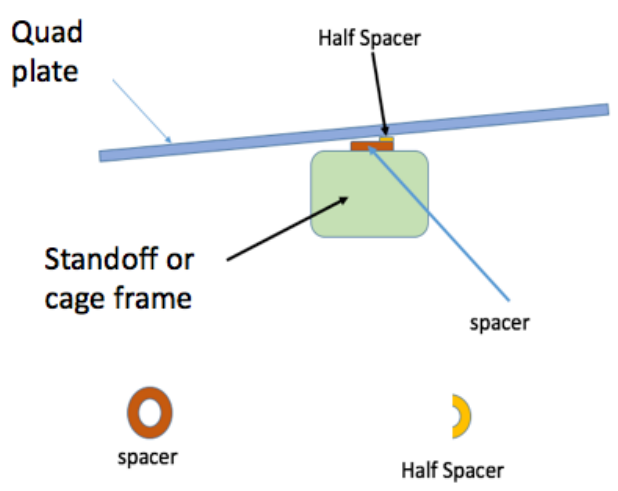

Figure 3.15: Spacers used to adjust the quad plate position.
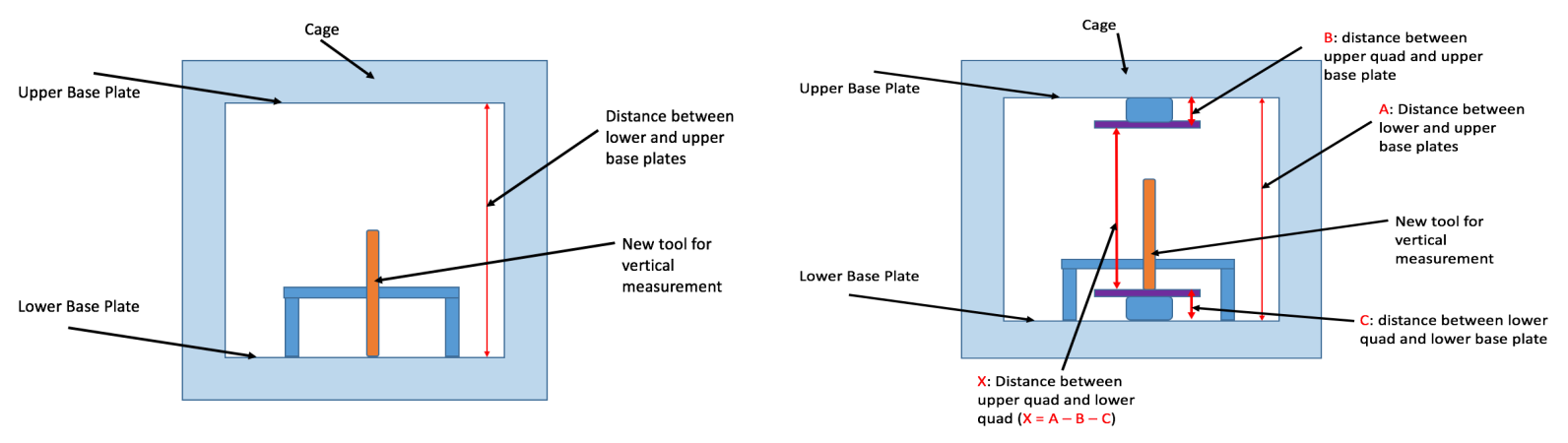

Figure 3.16: Conceptual diagram of about the micrometer tool for vertical electrodes alignment.

spacer (washer) between the standoff (or cage frame) and the plate, as shown in Fig. 3.15. The half spacer can introduce an angle which will affect nearby regions.

\section{Vertical Quad Plate Alignment:}

The concepts of vertical quad plate alignment are given in Fig. 3.16. As we learn from Fig. 3.16, to get the distance between two vertical electrodes $(X=A-B-C)$, we need know:

- A: distance between lower and upper base plates;

- B: distance between upper quad and upper base plate;

- C: distance between lower quad and lower base plate.

To perform the alignment, we need to know the expected (design) values of A, B and C. For details about the design drawing, see Ref. [75]. Some related values are shown in Fig. 3.17. As an example, we show results for one quad plate alignment before and after adjustment in Fig. 3.18. 


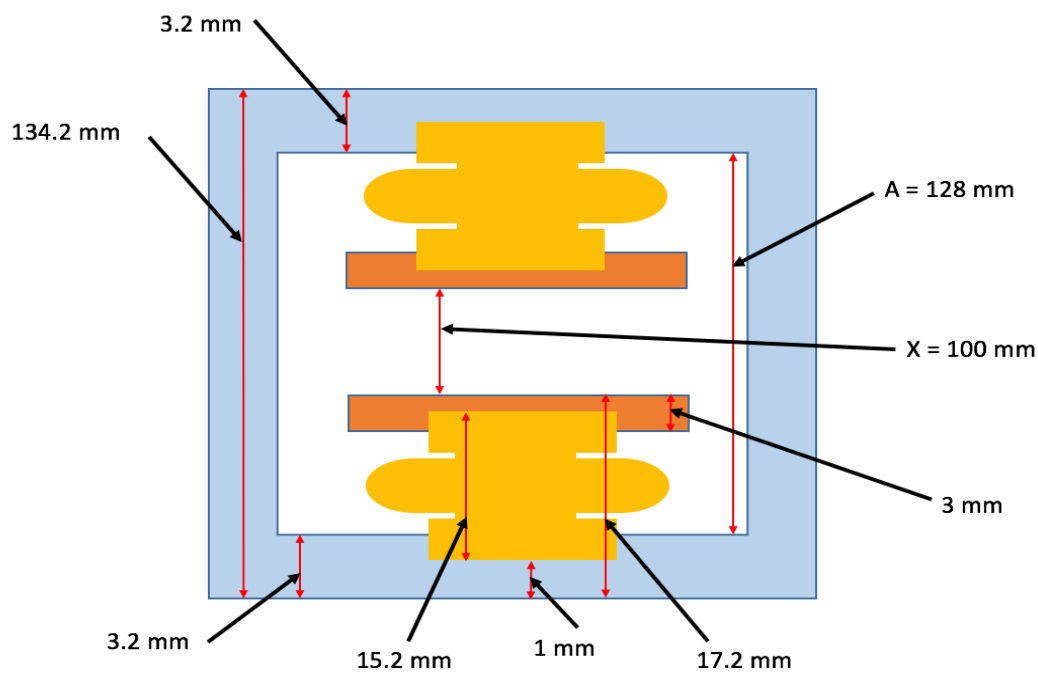

Figure 3.17: Cartoon with designed values for vertical quad plate alignment.

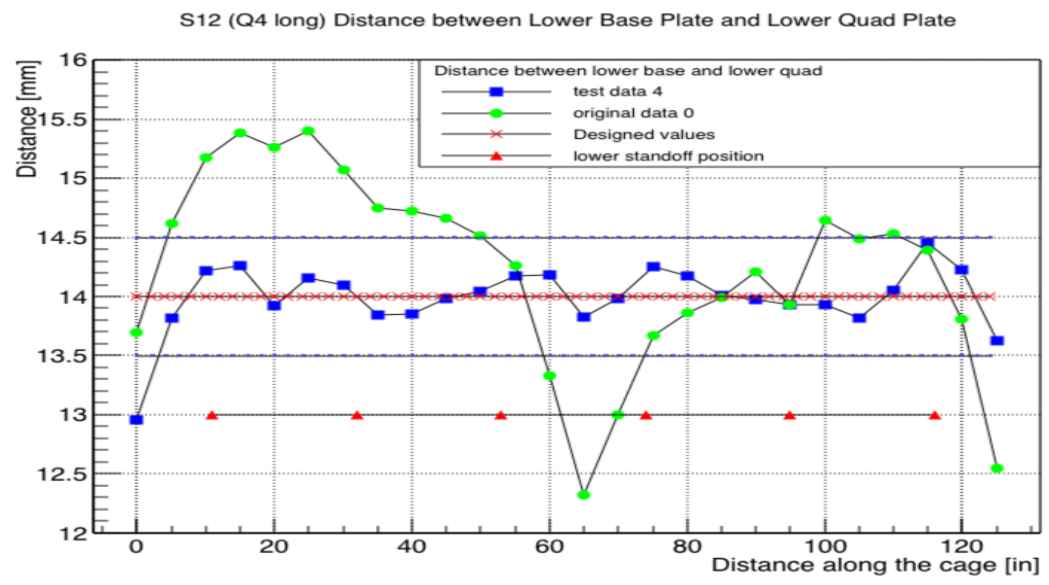

Figure 3.18: S12 (Q4 long) distance between lower base plate and lower quad plate (Green data is before shimming; Blue data is after shimming (final); design value is $14 \mathrm{~mm}$ ). 


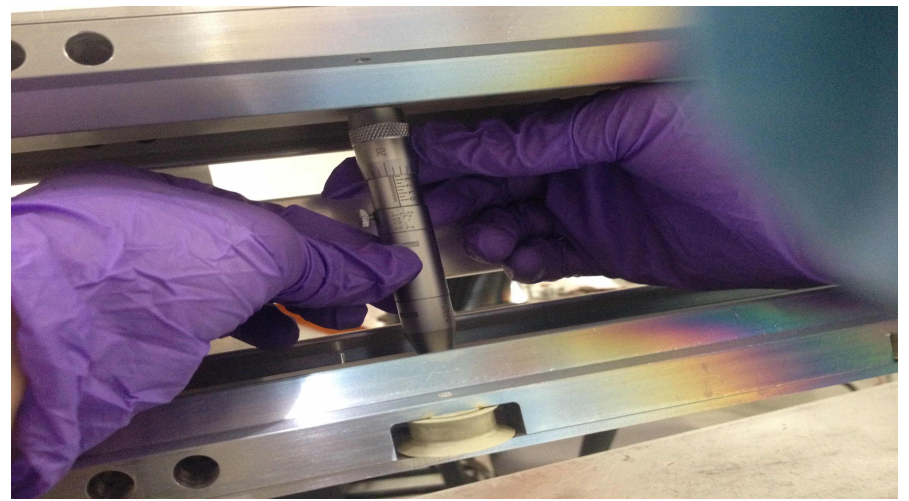

Figure 3.19: Micrometer tool for measuring the gap distance.

The vertical gap between two plates can be measured by a different micrometer tool as shown in Fig.3.19. The quad plate alignment procedure is discussed in detail in Ref. [76]. Based on the concepts shown in Fig. 3.16, we provide a summary of the vertical quad alignment measurement below:

1. Measure the vertical base gap (A) with the tool in Fig. 3.19;

2. Measure all lower/upper standoffs with caliper;

3. Measure the lower/upper base plate with the tool in Fig. 3.16;

4. Measure the lower/upper quad plate with the tool in Fig. 3.16;

5. Use results from steps 3 and 4 to calculate and plot the distance between lower/upper base plate and lower/upper quad plate, then compare with the design values (see Fig. $3.18)$;

6. If the measured value in step 5 is larger than the design value, remove the spacer (if spacer exists) and/or replace the standoff with a smaller height; if the measured value in step 5 is smaller than the design value, add spacer and/or replace the standoff with a larger height. (Note we can add spacer to both sides of the standoff);

7. Repeat steps 4, 5 and 6 , until we can produce a plot which shows that the average measured values differ from the design value less than $\pm 0.5 \mathrm{~mm}$ (for individual values, the difference should be less than $\pm 2 \mathrm{~mm}$ );

8. Calculate and plot the vertical quad gap $(X=A-B-C)$.

\section{Horizontal Quad Plate Alignment:}

The horizontal quad plate (inner/outer) alignment uses the micrometer tool shown in Fig. 3.14. Horizontal quad plate alignment is based on the vertical quad alignment, which means the horizontal quad plate's position is relative to that of the vertical quad plates. The 

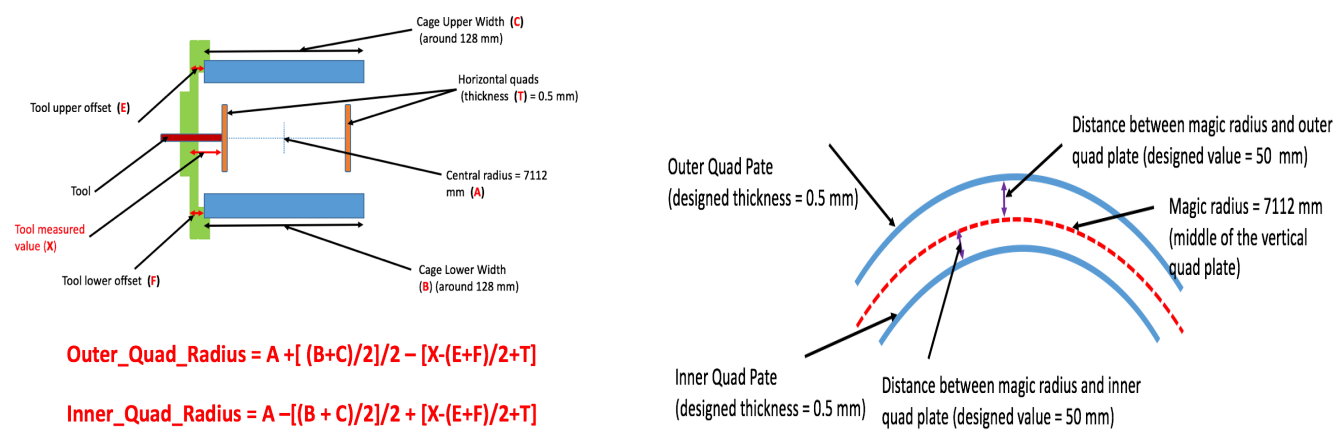

Figure 3.20: Horizontal quad plate alignment.

measurement concept is very similar to the vertical electrodes alignment and is given in Fig. 3.20 .

Similarly, we can summarize the horizontal quad plate alignment steps:

1. Measure all outer/inner standoffs with caliper;

2. Measure the lower/upper cage width (along the cage) with caliper and calculate their average values;

3. Measure the lower/upper tool offset with caliper and calculate their average;

4. Assume the thickness of the horizontal quad plates is uniform and equals to $0.5 \mathrm{~mm}$;

5. Measure the distance of the quad plate to tool zero base;

6. Use the values in steps $2,3,4$ and 5 to calculate the quad radius;

7. If the measured value in step 6 is larger than the design value, remove the spacer (if spacer exists) and/or replace the standoff with a smaller height; if the measured value in step 6 is smaller than the design value, add spacer and/or replace the standoff with a larger height;

8. Repeat steps 5 and 6 , until we can get a plot which shows that the measured values meet the requirements;

9. Calculate the horizontal gap and compare it with design values, if the measured gaps are beyond the specs, repeat step 8.

\subsubsection{Laser Alignment System}

The alignment group at Fermilab has a laser alignment system shown in Fig. 3.21, which can scan the detailed coordinates of the plate surface. Although those coordinates $(x, y, z)$ are in a random coordinate system, they can tell us the deviations from the designed position when analyzed carefully (see Fig. 3.22). The laser scan data can also be organized 


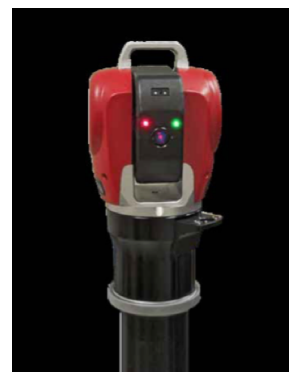

Figure 3.21: Laser alignment system for quad plate alignment.

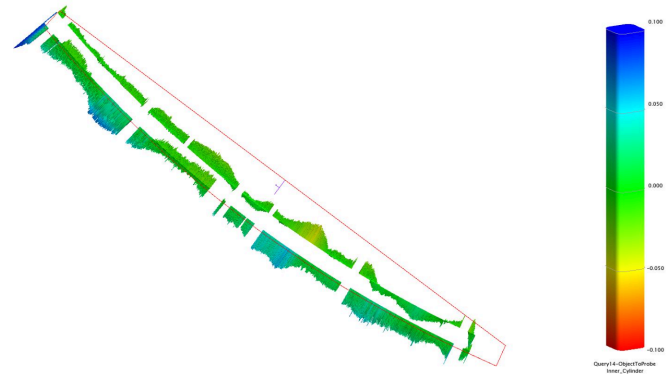

Figure 3.22: Laser scan data for inner and outer plates from H. Friedsam (top view).

in a ring cylindrical coordinate system, and all quad plates are considered in the global analysis discussed in Ref. [77].

To compare the laser scan data with our micrometer tool data, we need to fit the laser scan data to either a circle or a cylinder. Because the plates and/or the cage may twist, the fitted radius may not equal the radius of the ring and the fitted base plane may not sit on the ring plane. We prefer fitting a cylinder to reduce the twist effects. The detailed technique of fitting the laser scan data is discussed in Ref. [78].

We should point out that the micrometer tools only measure the middle part of the plates, while the laser alignment system can scan the entire surface of the plate. As an example, we show the comparison between the two methods in Fig. 3.23.

\subsubsection{Q1 Outer Plate Alignment}

The ESQ Q1 is positioned immediately after the injection point. Its particular position means the Q1 outer plate plays an important role in storing muons. The Q1 outer plates and support insulators are estimated to have reduced the stored muon population by about $40 \%$ [12]. Different modifications for Q1 have been considered, e.g., relocating the Q1 outer plate from $\mathrm{x}=5 \mathrm{~cm}$ to $\mathrm{x}=7 \mathrm{~cm}$ to allow for the uninhibited injection of the muon beam. In such a case, one may expect a higher voltage will be required to maintain the field strength. The current plan is to reduce the amount of interaction material by using a "massless" mylar plate at the design position $(\mathrm{x}=5 \mathrm{~cm})$, as shown in Fig. 3.24, which will allow us to store 


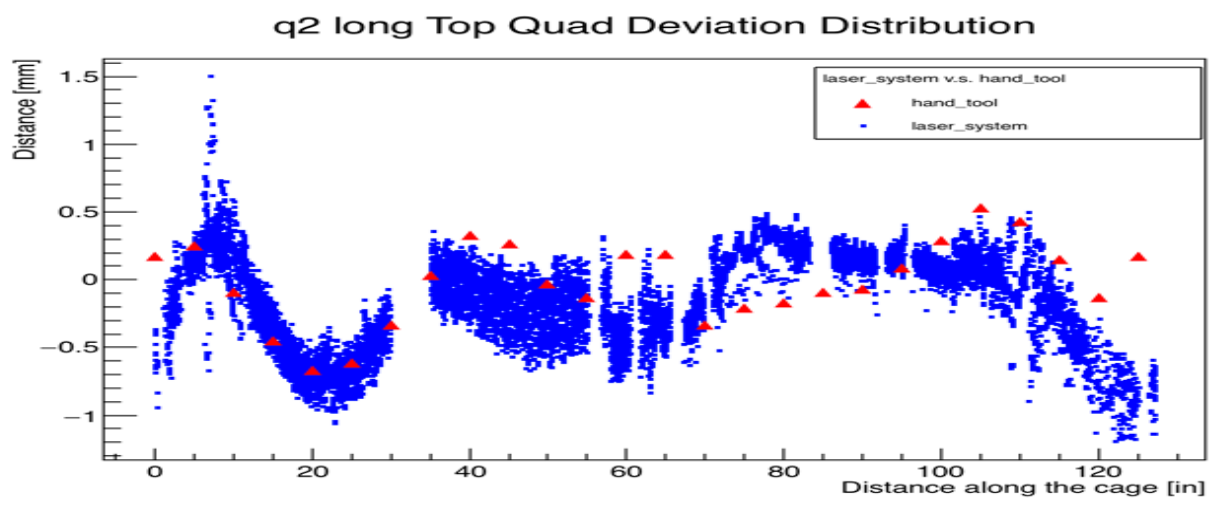

Figure 3.23: Q2L top quad plate deviation distribution: Blue is laser scan data and Red is micrometer tool data.

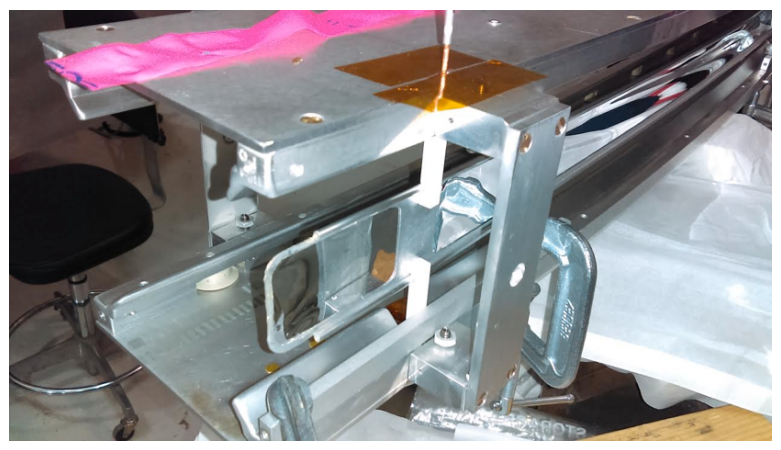

Figure 3.24: Q1 outer "Mylar" plate.

more muons [79].

The new mylar plate has a thickness of around $0.025 \mathrm{~mm}$. Hence, we could not use the previous standoffs to hold the plate horizontally. Instead, we modify our cage and rails to use two new standoffs to hold the plate vertically as shown in Fig. 3.24. There are two major challenges to achieving the alignment goals with the mylar plate: first, the plate is very brittle and we have to align it very carefully in order not to damage it; second, there exists a large waviness along the plate, which can result in deviations as large as $\pm 4 \mathrm{~mm}$.

Fig. 3.25 shows the concepts for the alignment. Because of the challenges we are facing, the alignment of the Q1 outer plates is not as accurate as the other ESQ alignments. For example, the edge of the plates is not uniform, the cage may be twisted, and the surface of mylar has some waviness which is difficult to remove. However, after all attempts to minimize deviations, a dry run with the trolley shows the alignment is acceptable. Results are shown in Appendix A. Because the new standoffs for Q1 outer plates are slightly shorter than expected, we also need to apply a vertical alignment. The concepts of the vertical alignment is shown in Fig. 3.26. 


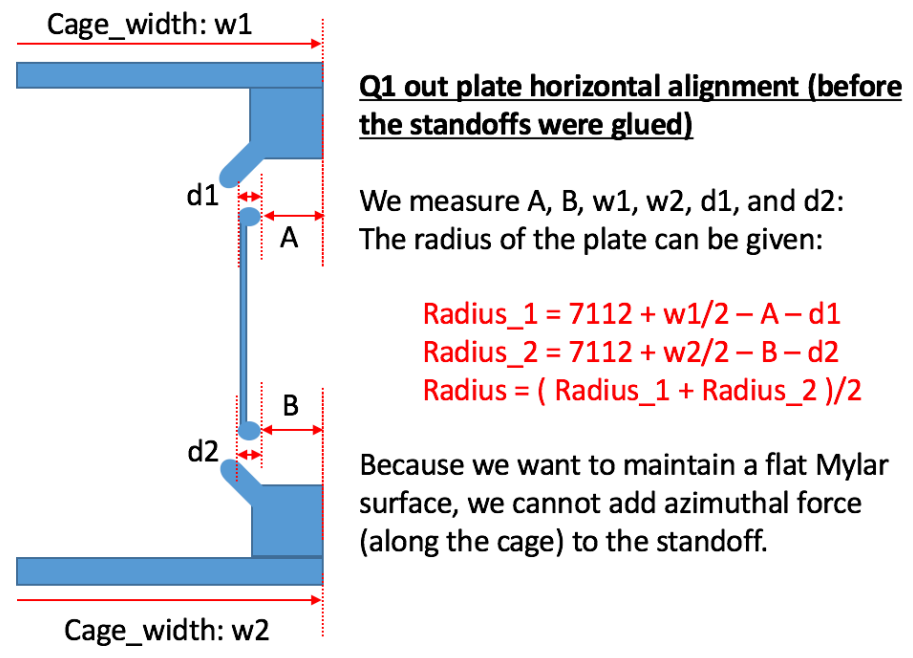

Figure 3.25: Schematic of Q1 outer plate alignment.

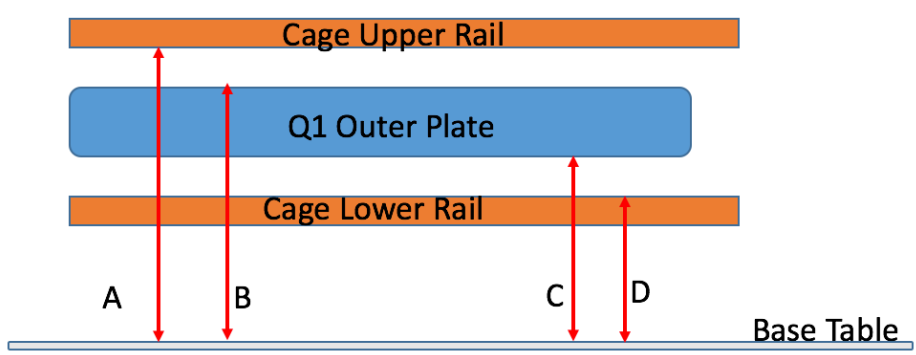

For Q1 outer plate vertical alignment, we use the distances between cage rails and plate edges.

$$
\begin{aligned}
& \text { Distance_1 }=A-B\left(=A^{\prime}-B^{\prime}+L\right) \\
& \text { Distance_2 }=C-D\left(=C^{\prime}-D^{\prime}+L\right)
\end{aligned}
$$

Here, $A^{\prime}, B^{\prime}, C^{\prime}$ and $D^{\prime}$ are modified distances; $L$ is the width of the arm of the standing caliper.

$$
\begin{array}{ll}
A^{\prime}=A-L ; & B^{\prime}=B \\
C^{\prime}=C-L ; & D^{\prime}=D
\end{array}
$$

We measure:

Distance_1' $=A^{\prime}-B^{\prime}$

Distance_2' $=C^{\prime}-D^{\prime}$

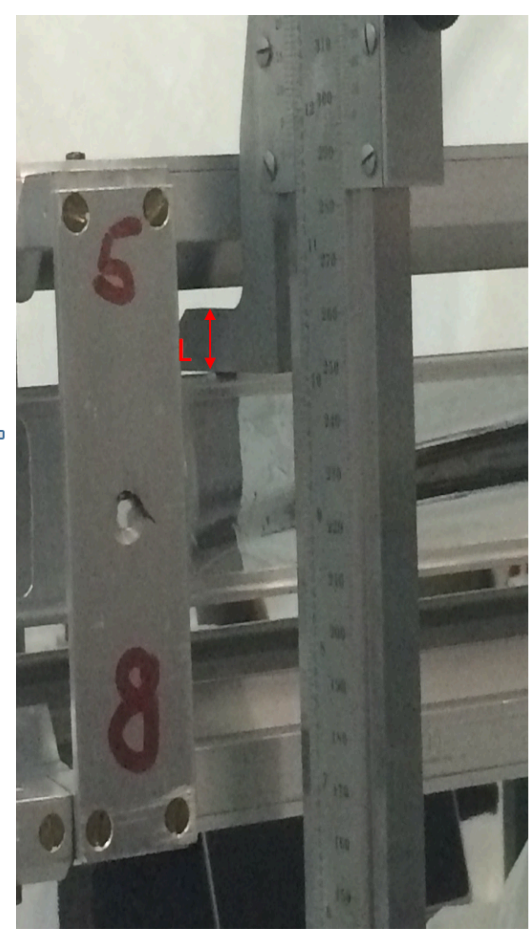

Figure 3.26: Schematic of Q1 outer plate vertical alignment. 


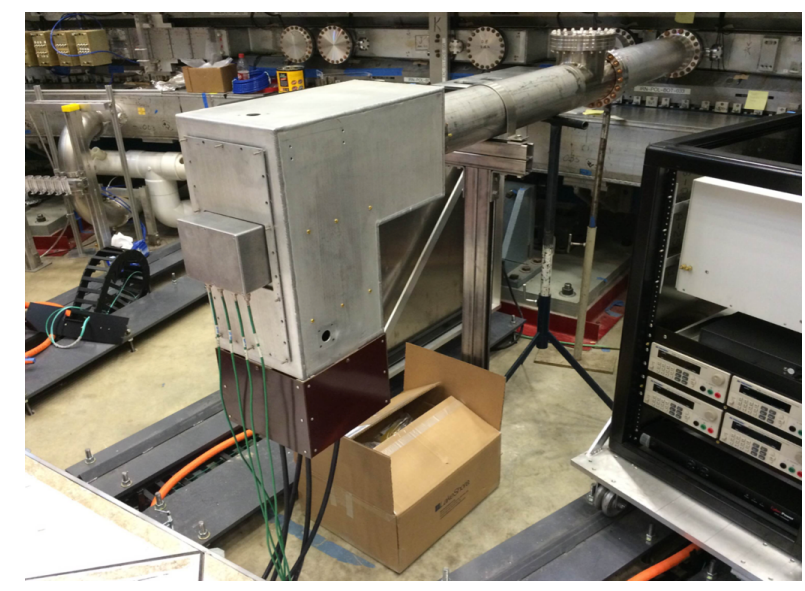

Figure 3.27: Example of a "successfully" installed quad extension.

\subsubsection{Final Results of Quad Plate Alignment}

The major contributions of this dissertation work to the quad plate alignment include the quad plate survey with the Capacitec tool, the plate alignment with the micrometer tool and analyzing the laser scan data. The final results of the quad plate alignment are summarized in the E989 UW Elog, where we also present the comparison between the micrometer tool results and the laser alignment scan results. All the ESQ plates have been aligned within specifications. The final results of our alignment are provided in Appendix A.

\subsection{Quad Extension and Readout Systems}

In the experiment, we use a quad extension for each quad to bridge the power supply and the quad plates. One of the quad extensions is shown in Fig. 3.27. The extension keeps the HV feed-through components away from trapped electrons inside the vacuum chamber. It contains the connection leads, extension tube, and a feed-through box.

\subsubsection{Installation of Quad Extension}

The installation of the quad extension is very challenging. Several people have to work together to make sure the parts are not damaged. The connection leads bridge the quad plates and extension tubes. The distance between a lead and a case wall and the distance between a lead and another lead must be larger than $1 \mathrm{~cm}$ to avoid high voltage sparks. See Fig. 3.28.

The connection leads are held by a macor "batman" as shown in Fig. 3.29 (left). We use buttons to lock the leads at the end taps of the batman. The extension tubes are inside an extended chamber as shown in Fig. 3.29 (right). The feed-through box contains HV resistors and a spark circular diagram, and is installed at the end of the extension chamber as shown in Fig. 3.30. 


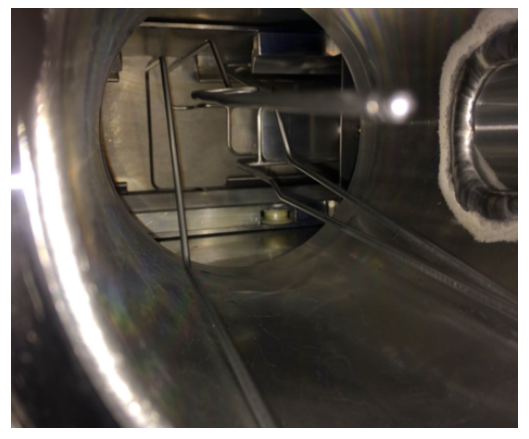

Figure 3.28: Example of installing the connection leads.
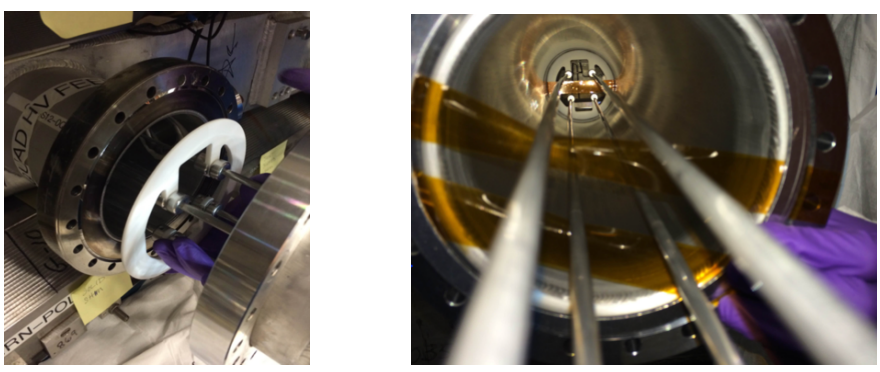

Figure 3.29: Quad Batman (right); Extension tubes (left).

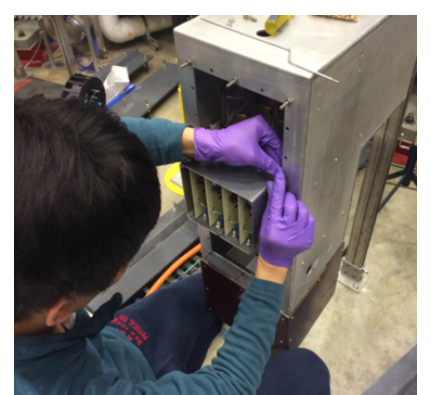

Figure 3.30: Feed-through box. 

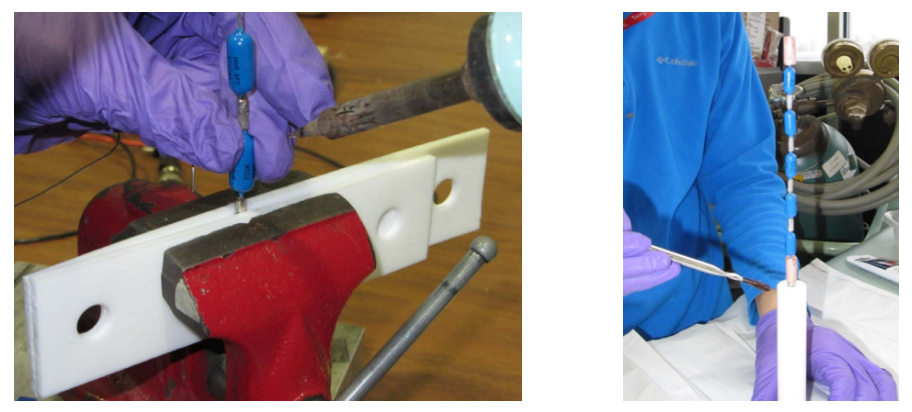

Figure 3.31: Preparation of HV resistor.
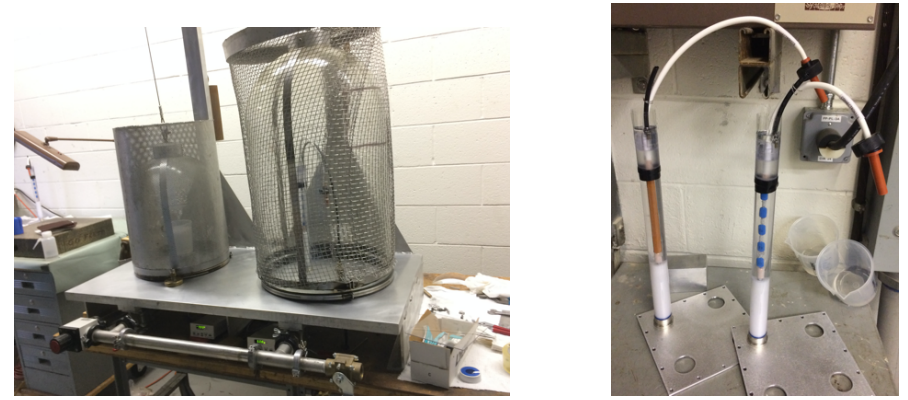

Figure 3.32: Potting resistors: Vacuum bell jar (left); potted resistors (right).

\subsubsection{Potting Resistors}

We plant the HV resistors inside the feed-through box to suppress sparks. The HV resistors also provide the resistor for the quad plate $\mathrm{RC}$ time constants. For each quad plate, the required resistor value is determined by the quad plate capacitance measurement. Therefore, we need $4 \times 8=32$ resistors in total. Potting resistors is time-consuming and only 1-2 resistors could be potted per day due to limited available manpower.

The resistors are prepared as shown in Fig. 3.31. They are potted inside a vacuum bell jar as shown in Fig. 3.32(left). Fig. 3.32 (right) shows the potted resistor examples. The details of potting resistors can be found in Ref. [80].

\subsubsection{Quad Readout}

The electrical diagram of the ESQ High Voltage (HV) pulsing systems is given in Fig. 3.33. The concept of this control electronics for high voltage (HV) pulses is discussed in Ref. [81]. In E989, we use two types of HV pulses: one step charge pulse and two step charge pulse. The latter one is mainly used for the scraping procedure at the beginning of muon storage inside the ring. In this section, we present the time diagram of the control pulses, the circuits of the control pulses and spark detection.

The time diagram of control pulses is discussed in Ref. [81], and its concept is shown in Fig. 3.34. We will derive the external trigger from a copy of accelerator control signals, 


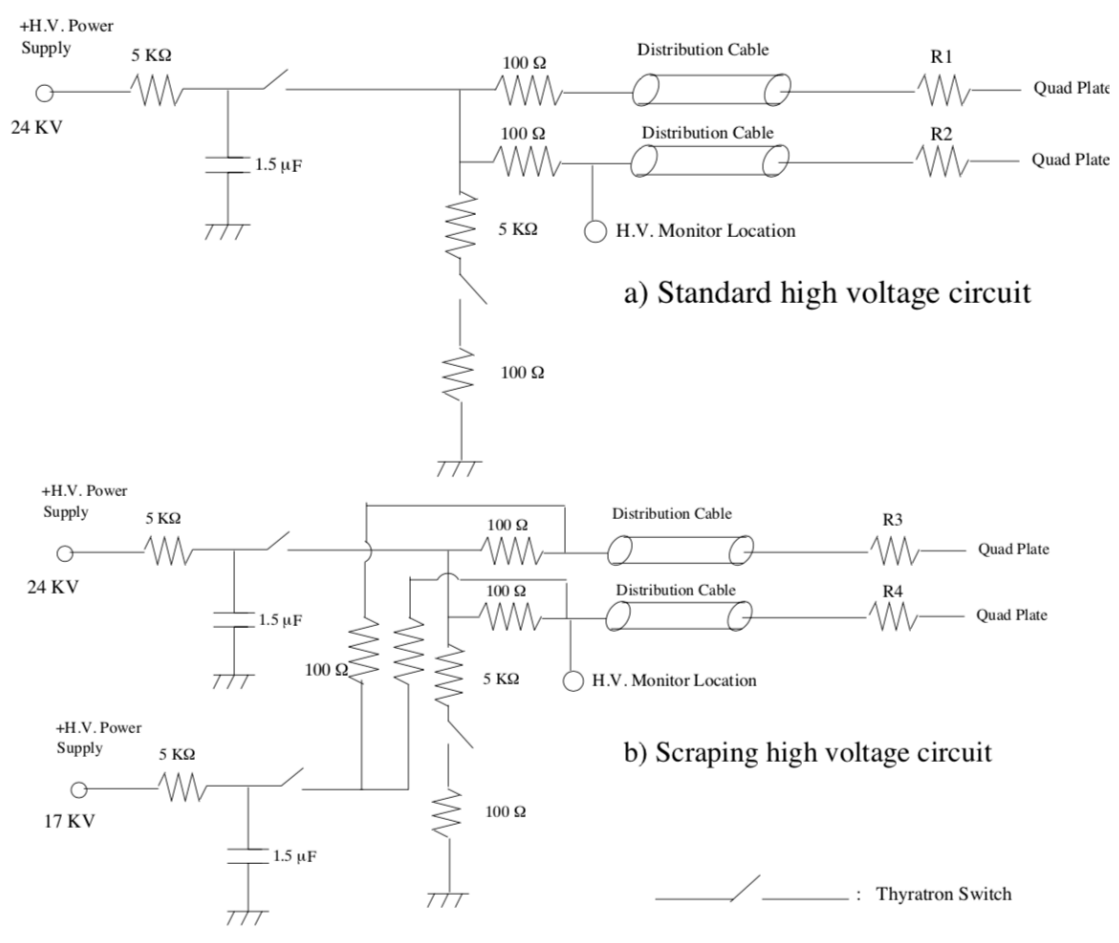

Figure 3.33: Schematic diagram of the scraping and standard HV pulsing systems.

which indicate the beginning of the beam injection into the muon storage ring. Then we can use that as our time baseline to set the timing width for charge and discharge for our two types of HV pulses.

During the experiment, the plates are charged prior to each fill and the voltage will be held constant throughout the measuring period. However, if electrons, for example, produced by field emission or gas ionization, ionize the residual gas, they may cause a spark at the end, and then discharge the plates. The electronics control system should be able to detect this and stop the pulses. The modules we use in the circuits of control pulses and spark detection are:

- BNC 8010: Pulse Generator

- LRS 428F: Linear FAN-IN/FAN-OUT MODULE

- LRS 821: Quad Discriminator

- LRS 365AL: Dual 4-Fold Logic

- LRS 222: Dual gate generator

- LRS 429: Logic FAN-IN/FAN-OUT

- PHILLIPS 726: ECL/NIM/TTL Translator 


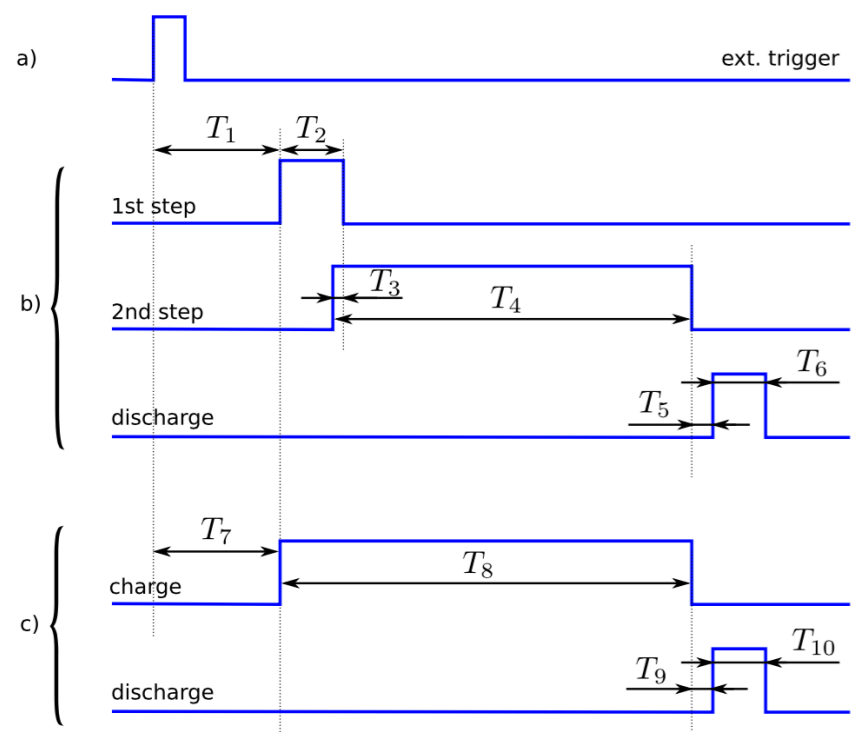

Figure 3.34: Time diagram of control pulses.

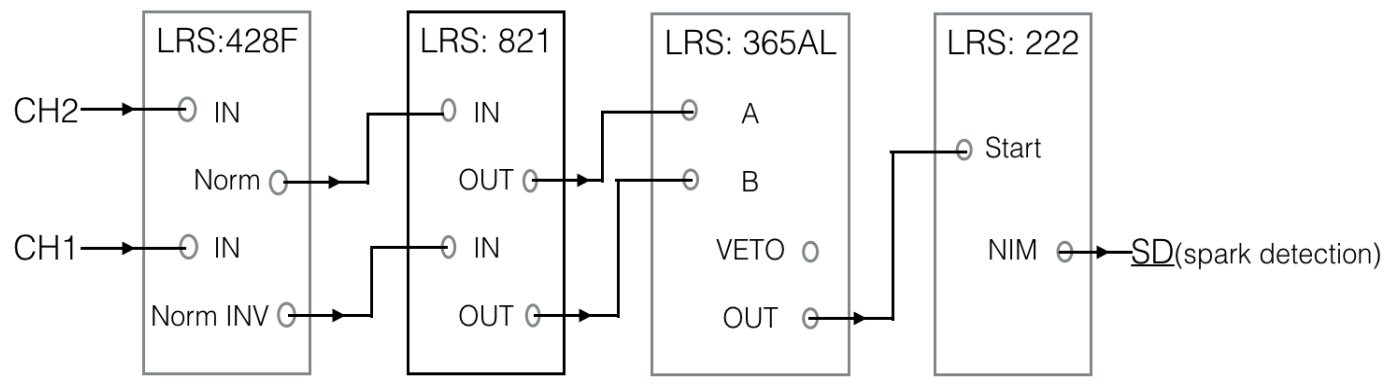

Figure 3.35: Spark detection circuits.

Fig. 3.35 shows our circuit diagram for spark detection. As an illustration, we only consider two channels in Fig. 3.35: $\mathrm{CH} 1$ and $\mathrm{CH} 2$, where $\mathrm{CH} 1$ is for the vertical plates and $\mathrm{CH} 2$ is for the horizontal plates. For the experiment, we should have four channels for our four plates. The NIM output of the spark detection (SD) circuits, will tell the electronics system that there is a spark.

In Fig. 3.36, we use a pulse generator to produce an external trigger. The circuits contains details as shown in Fig. 3.34. We use a dual gate generator (LRS 222) to set the delay time and the pulse width for charge step and discharge step. The output TTL signals of the charge/discharge procedure will be sent to the HV station, from which we start to charge or discharge the plates.

We can also use a count display (CD) to count the number of pulses and how many sparks we detect. The relevant circuit diagram is given in Fig. 3.37. This will help us to realize and understand the spark situation directly.

For the experiment, we can build the entire control pulse system in one simple module, as shown in Fig. 3.38. We name it "Sten's module" after its author - Sten Hansen. By using some Python scripts, we can remotely turn on or turn off this module. We can also reset our time width for each step shown in Fig. 3.36. 

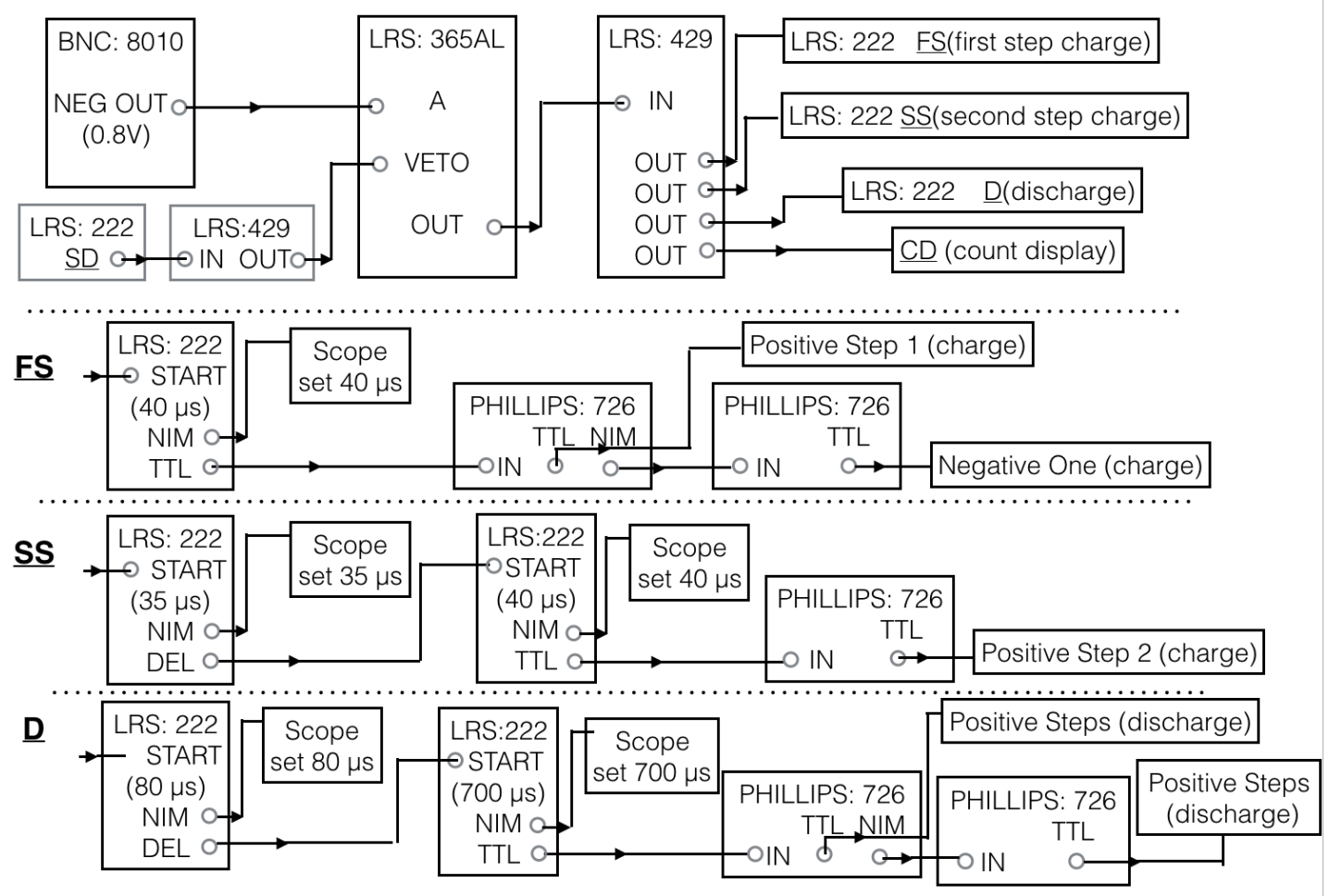

Figure 3.36: Circuits of control pulses and spark detection with details.

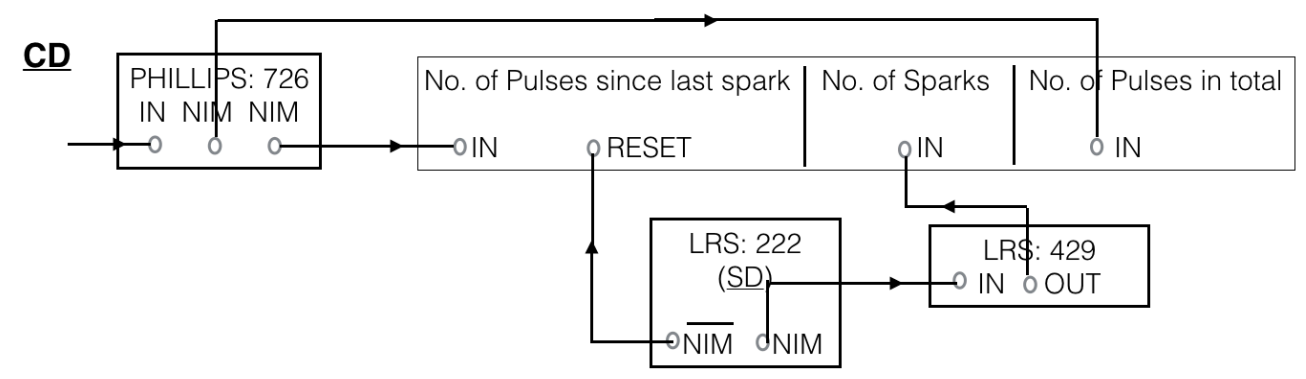

Figure 3.37: Circuits for pulse count display.

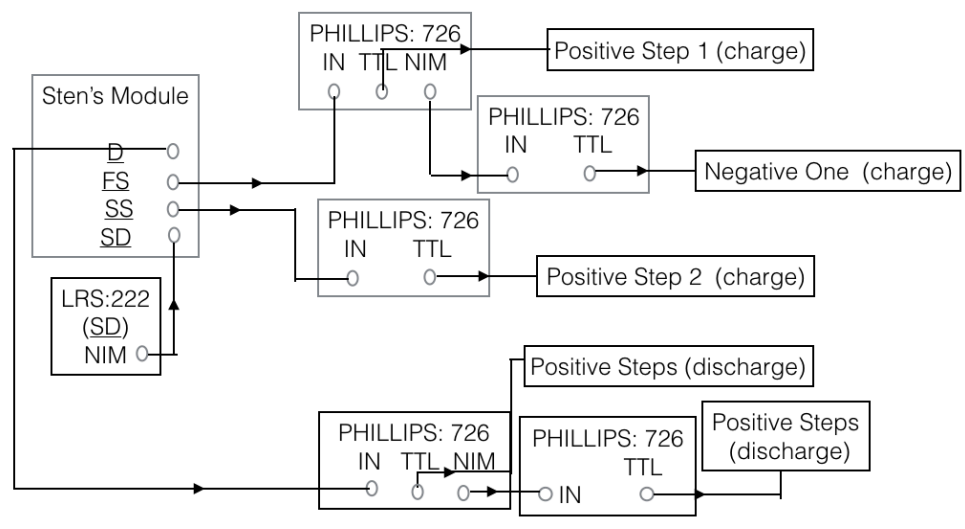

Figure 3.38: Circuits of control pulses and spark detection with Sten's module. 
The CAMAC (Computer Automated Measurement and Control) is an old standard developed in the 1970s. We use a CAMAC based data acquisition system (DAQ) to perform our spark analysis. By taking advantage of its high channel count and high speed, we can monitor our 32 plates (in total) through 32 channels and then study the spark forms which will give a high number of oscillations into the the scaler. The concept of the CAMAC DAQ system is shown in Fig. 3.39.

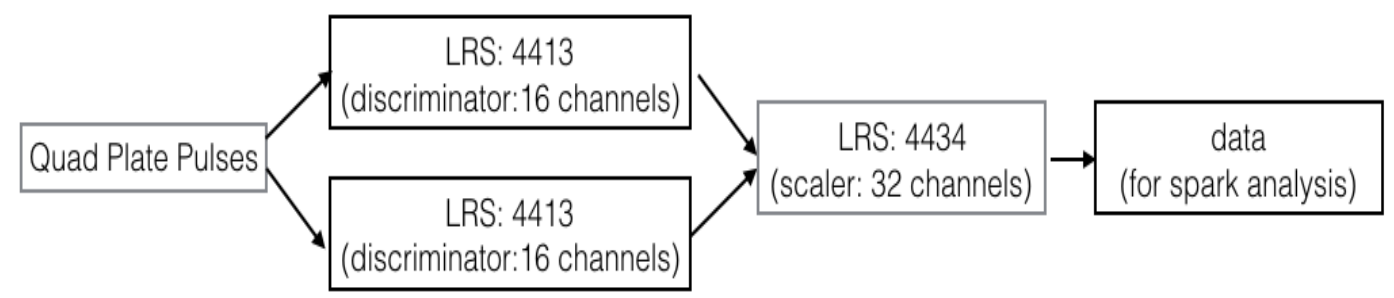

Figure 3.39: CAMAC DAQ scheme for spark analysis.

We successfully installed all eight quad extensions and the corresponding readout systems, where I played a leading role in the installation of the quad extensions and potting resistors. I also made a significant contribution to the quad readout system and helped with the ESQ operation to test the whole system in the vacuum environment. The ESQ system is very critical to the experiment. The plate alignment, together with the quad extension and readout system, plays a significant role in storing the muon beam and is very important to achieve the E989 measurement goal by impacting both the statistical (in a confined run period) and systematic errors. 


\section{CHAPTER 4}

\section{BEAM DYNAMICS IN THE MUON STORAGE RING}

The Fermilab Muon $g-2$ Experiment continues to use the storage ring built for the BNL $g-2$ experiment. The field index $n$ of a storage ring with dipole magnetic field $B_{0}$ and electric gradient $\partial E_{R} / \partial R$ is defined as

$$
n(s)=\frac{R}{\beta B_{0}} \frac{\partial E_{R}(s)}{\partial R}, \quad \beta=v / c
$$

where $s$ is the longitudinal coordinate, and therefore, $\phi=s / R$ is the azimuth. This definition can be explained by the equation of motion.

In reality, not all the muons can travel around the ideal orbit (see Fig. 3.2). To understand the behavior of the muons, we must study the beam dynamics in the $g-2$ storage ring. In this chapter, we will discuss the beam dynamics and beam related systematic errors.

\subsection{Equation of Motion}

The general study of the equation of motion and beam dynamics is discussed in several textbooks $[82,83]$. In this section, we just consider the situation in the Muon $g-2$ storage ring. We assume that the dipole magnetic field is uniform. The reference orbit and coordinate system are given in Fig. 4.1, where we define a local Cartesian coordinate system moving along with the particle. For any path length $s$ along the trajectory, the position of the particle can be expressed as

$$
\vec{R}=r \hat{x}+y \hat{y}
$$

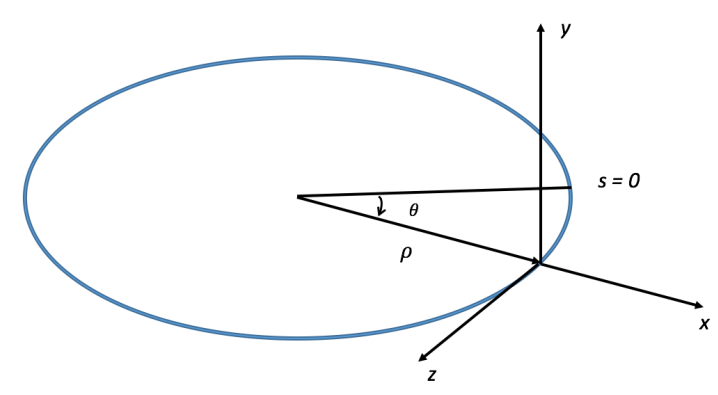

Figure 4.1: Reference orbit and coordinate system. 
where $r \equiv \rho+x$ and $\rho$ is the "magic" radius. The electromagnetic force applied to a moving particle with charge $e$ is given by

$$
\vec{F}=e \vec{E}+e \vec{v} \times \vec{B}=e\left(E_{x}-v_{z} B_{0}, E_{y}, E_{z}+v_{x} B_{0}\right)
$$

with our assumption that $\vec{B}=B_{0} \hat{y}$. Moreover, we only consider the 2-dimensional electric gradient $\left(E_{z}=0\right)$ and $F_{z} \simeq 0$ with the fact that $v_{s} \ll v_{z}$.

The equation of motion is

$$
\vec{F}=\frac{d \vec{p}}{d t}=\gamma m \ddot{\vec{R}}
$$

where $\gamma=\left(1-\beta^{2}\right)^{-1 / 2}=1 / \sqrt{1-(v / c)^{2}}$. To investigate this, we need to evaluate $\ddot{\vec{R}}$ in our coordinates

$$
\begin{aligned}
\dot{\vec{R}} & =\dot{r} \hat{x}+\dot{y} \hat{y}+r \dot{\theta} \hat{z} \\
\ddot{\vec{R}} & =\left(\ddot{r}-r \dot{\theta}^{2}\right) \hat{x}+\ddot{y} \hat{y}+(2 \dot{r} \dot{\theta}+2 \ddot{\theta}) \hat{z}
\end{aligned}
$$

Now we can derive the components of the equation of motion.

\section{$\hat{x}$-direction:}

We have

$$
\ddot{r}-r \dot{\theta}^{2}=\frac{e\left(E_{x}-v_{z} B_{0}\right)}{\gamma m}
$$

Since $v_{x, y} \ll v_{z}$, the momentum of the particle approximately equals $\gamma m v_{z}$. Using the relations $B_{0} \rho=p / e, \dot{\theta}=v_{z} / r, d / d t=\frac{d s}{d t} \frac{d}{d s}$ and $r=\rho+x$, we have

$$
\frac{d^{2} x}{d s^{2}}-\frac{\rho+x}{\rho^{2}}=\frac{E_{x}-v_{z} B_{0}}{v_{z} B_{0} \rho}\left(1+\frac{x}{\rho}\right)^{2}
$$

For a symmetric electric field gradient in 2-dimension, we have

$$
E_{x}=E_{x}(0,0)+\frac{\partial E_{x}}{\partial x} x+\frac{\partial E_{x}}{\partial y} y=\frac{\partial E_{x}}{\partial x} x=\frac{\partial E_{R}}{\partial R} x
$$

Here, $E_{x}(0,0)=0$ and $\frac{\partial E_{x}}{\partial y}=0$. Therefore,

$$
\frac{d^{2} x}{d s^{2}}-\frac{\rho+x}{\rho^{2}}+\frac{1}{\rho}\left(1+\frac{x}{\rho}\right)^{2}-\frac{1}{v_{z} B \rho} \frac{\partial E_{R}}{\partial R}\left(1+\frac{x}{\rho}\right)^{2} x=0
$$

Since $x \ll \rho$, we can neglect the higher order $(x / \rho)$ terms. After some mathematical steps, we can get

$$
\frac{d^{2} x}{d s^{2}}+\frac{1}{\rho^{2}}\left[1-\frac{\rho}{v_{z} B_{0}} \frac{\partial E_{R}}{\partial R}\right] x=0
$$


Here we can define the field index (note that the definition here is slightly different from that in Eq. 4.1)

$$
n=\frac{\rho}{v_{z} B_{0}} \frac{\partial E_{R}}{\partial R}
$$

Then we can rewrite the equation of motion in the $\hat{x}$-direction w.r.t the azimuth $(d s=\rho d \theta)$ :

$$
\frac{d^{2} x}{d \theta^{2}}+(1-n) x=0
$$

\section{$\hat{y}$-direction:}

Similarly, we have

$$
\ddot{y}=\frac{e E_{y}}{\gamma m}=\frac{E_{y} v_{z}}{B_{0} \rho}
$$

The way to obtain the final form of the equation of motion is very similar to that of the equation of motion in the $\hat{x}$-direction. So, we will have

$$
E_{y}=E_{y}(0,0)+\frac{\partial E_{y}}{\partial x} x+\frac{\partial E_{y}}{\partial y} y=\frac{\partial E_{y}}{\partial y} y=-\frac{\partial E_{R}}{\partial R} y
$$

and

$$
\ddot{y}-\frac{v_{z}}{B \rho} \frac{\partial E_{R}}{\partial R} y=0
$$

The equivalent form is

$$
\frac{d^{2} y}{d s^{2}}+\frac{1}{\rho^{2}}\left(\frac{\rho}{v_{z} B_{0}} \frac{\partial E_{R}}{\partial R}\right) y=0
$$

Using the definition of the field index $n$ in Eq. (4.13), we obtain

$$
\frac{d^{2} y}{d \theta^{2}}+n y=0
$$

Eq. (4.13) and Eq. (4.18) are both of the form of Hill's Equation:

$$
x^{\prime \prime}+K(s) x=0
$$

where the "spring constant" $K$ is a function of position $s$. In Ref. [82, 83], two methods of solution are discussed: one is called the piecewise method, which has similarities to the geometrical optics; the other is called the closed form method, which is based on the periodicity of $K(s)(K(s+C)=K(s))$.

The general solutions of our beam equations of motion are given by

$$
x=x_{e}+\sqrt{\epsilon_{x} \beta_{x}(s)} \cos \left[\nu_{x} \frac{s}{R}+\phi_{x}(s)\right]
$$




$$
\begin{gathered}
y=\sqrt{\epsilon_{y} \beta_{y}(s)} \cos \left[\nu_{y}+\phi_{y}(s)\right] \\
x_{e}(s)=R_{e}(s)-R_{0}=D(s) \frac{p-p_{0}}{p}, \quad c p_{0}=e B R_{0}
\end{gathered}
$$

Based on the lattice design of the $g-2$ ring, we shall be able to determine the beam dynamics by solving the equation of motion. To estimate the field corrections to the experiment, the general betatron oscillations and momentum dispersion have to be studied and calculated, as described in Ref. [68]. The detailed information can be found in Appendix B.

\subsection{Beam Related Systematic Errors}

In this section, we will discuss the beam related systematic errors. The behavior of the beam in a weak-focusing betatron and its injection features are important in the determination of $\omega_{a}$. Beam resonances in the storage ring can cause muon losses if the amplitude of the resonances is larger than the storage aperture, thus distorting the observed decay positron time spectrum, and therefore must be avoided when choosing the operating parameters of the ring. Also, the frequency of the "coherent betatron oscillation" (CBO) should be set carefully, because the frequency of the coherent radial beam motion lies close to the second harmonic of $f_{a}=\omega_{a} / 2 \pi$. See Table 4.1. If the frequency of CBO is too close to $2 f_{a}$, the beat frequency, $f_{C B O}-f_{a}$, complicates the extraction of $f_{a}$ from the data and can produce a significant systematic error [12]. In addition, because the detector acceptance for decay positrons depends on the radial coordinate of the muon at the point where it decays, the coherent radial motion of the stored muon beam can produce an amplitude modulation in the observed decay positron time spectrum. Moreover, because the muon beam has a radial momentum profile, the electrical field correction has to be evaluated. The vertical betatron motion, which will introduce the $\vec{\beta} \cdot \vec{B} \neq 0$ term, also affects the measurement of $a_{\mu}$. Thus, a relevant "pitch" correction is also necessary.

\begin{tabular}{lllll}
\hline \hline Physical frequency & Variable & Expression & Frequency & Period \\
\hline Anomalous precession & $f_{a}$ & $\frac{e}{2 \pi m} a_{\mu} B$ & $0.23 \mathrm{MHz}$ & $4.37 \mu \mathrm{s}$ \\
Cyclotron & $f_{C}$ & $\frac{v}{2 \pi R_{0}}$ & $6.71 \mathrm{MHz}$ & $149 \mathrm{~ns}$ \\
Horizontal betatron & $f_{x}$ & $\sqrt{1}-n f_{C}$ & $6.23 \mathrm{MHz}$ & $160 \mathrm{~ns}$ \\
Vertical betatron & $f_{y}$ & $\sqrt{n} f_{C}$ & $2.48 \mathrm{MHz}$ & $402 \mathrm{~ns}$ \\
Horizontal CBO & $f_{C B O}$ & $f_{C}-f_{x}$ & $0.48 \mathrm{MHz}$ & $2.10 \mu \mathrm{s}$ \\
Vertical waist & $f_{V W}$ & $f_{C}-2 f_{y}$ & $1.74 \mathrm{MHz}$ & $0.57 \mu \mathrm{s}$ \\
\hline \hline
\end{tabular}

Table 4.1: Important frequencies in the muon storage ring (for $n=1.37$ ) [44].

From the solutions of the equations of motion (see Eq. (4.13) and Eq. (4.18)), the horizontal and vertical tunes, as an approximation, are known as

$$
\nu_{x}=\sqrt{1-n} \quad \text { and } \quad \nu_{y}=\sqrt{n}
$$




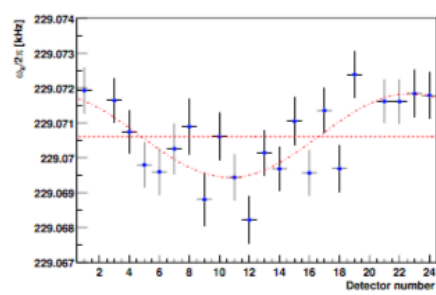

(a) No $\mathrm{CBO}$ in Fit

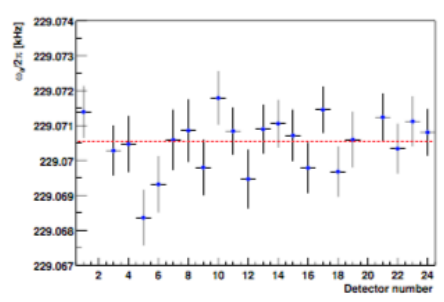

(b) $\mathrm{CBO}$ in Fit

Figure 4.2: The dependence of the extracted value of $a_{\mu}$ vs. detector number: (a) With no $\mathrm{CBO}$ in the fit function; (b) With CBO in the fit function [12].

BNL E821 used several $n$ values for its data acquisition: $n=0.137,0.142$ and 0.122 . For $\mathrm{n}=0.137$, the horizontal and vertical frequencies are $f_{x} \simeq 0.929 f_{C}$ and $f_{y} \simeq 0.37 f_{C}$. It is important that the betatron frequencies are not simple multiples of the cyclotron frequency, as this minimizes the effect of ring imperfections. Higher multipoles that drive resonances would also cause the muon losses in the storage ring.

The field index $n$ also affects the angular acceptance of the storage ring. For our $90 \mathrm{~mm}$ diameter storage region, the maximum horizontal and vertical angles of the muon momentum are given by

$$
\theta_{\text {max }}^{x}=\frac{x_{\max } \sqrt{1-n}}{R_{0}} \quad \text { and } \quad \theta_{\max }^{y}=\frac{y_{\max } \sqrt{n}}{R_{0}}
$$

where $x_{\max }=y_{\max }=45 \mathrm{~mm}$. If the betatron oscillation amplitudes $A_{x}$ and $A_{y}$ are less than $45 \mathrm{~mm}$, the acceptance angle is reduced.

In our storage ring, we choose four-fold symmetry of the quadrupoles for beam vertical focusing mainly for two reasons: first, some quadrupole-free regions are needed for other subsystems, like Kicker, Tracker, Fiber Harps, etc.; second, the benefit of four-fold symmetry reduces the peak-to-peak betatron oscillation amplitudes, with $\sqrt{\beta_{\max } / \beta_{\min }}=1.03[12]$.

\subsection{1 $\mathrm{CBO}$}

The presence of the CBO was first noticed in BNL E821 from a plot that showed an azimuthal variation in the value of $a_{\mu}$, as shown in Fig. 4.2(a). This azimuthal dependence disappears when we include CBO. It is hidden when all detectors are added together because the $\mathrm{CBO}$ wavelength is just slightly larger than the ring circumference. This might be a hint to allow us to remove CBO by adding all detectors together (Actually, this is one of the techniques used in E821).

The frequency that a single fixed detector sees the beam coherently moving back and forth radially is the horizontal "Coherent Betatron Frequency", $f_{C B O}=f_{C}-f_{x}$. We may also view the ring as a spectrometer where the inflector exit is imaged at each successive betatron wavelength, as shown in Fig. 4.3. The CBO frequencies can also be found by doing a Fourier transform to the residuals from a fit to the five-parameter function (see Eq. (2.17)) 


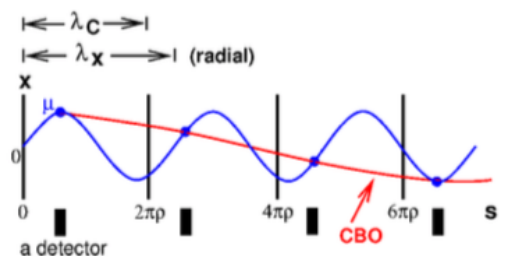

Figure 4.3: A cartoon to understand the CBO: horizontal (radial) $\mathrm{CBO}$ is shown in blue.

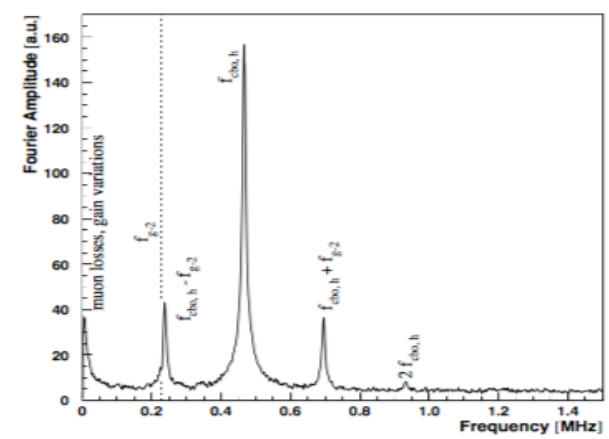

Figure 4.4: The Fourier transform to the residuals from a fit to the five-parameter function.

as shown in Fig. 4.4.

CBO can affect both the spin motion of the muon and the acceptance of the decay positron. Therefore, the systematic error from the $\mathrm{CBO}$ should be evaluated. The spin precession for the muon with and without $\mathrm{CBO}$ is shown in Fig. 4.5. The amplitude of the additional spin precession is about $10^{-4}$ times the amplitude of $g-2$ spin precession. A more complex form of the decay positron count, which includes muon loss function and $\mathrm{CBO}$ frequency is given by

$$
N(t)=\frac{N_{0}}{\gamma \tau_{\mu}} e^{-t / \gamma \tau_{\mu}} \cdot \Lambda(t) \cdot V(t) \cdot B(t) \cdot C(t) \cdot\left[1-A(t) \cos \left(\omega_{a} t+\phi(t)\right)\right]
$$

where

$$
\begin{aligned}
\Lambda(t) & =1-A_{\text {loss }} \int_{0}^{t} L(t) e^{-t / \gamma \tau_{\mu}} d t \\
V(t) & =1-e^{-t / \tau_{V W}} A_{V W} \cos \left(\omega_{V W} t+\phi_{V W}\right) \\
B(t) & =1-A_{b r} e^{-t / \tau_{b r}} \\
C(t) & =1-e^{-t / \tau_{C B O}} A_{1} \cos \left(\omega_{C B O} t+\phi_{1}\right) \\
A(t) & =A\left(1-e^{-t / \tau_{C B O}} A_{2} \cos \left(\omega_{C B O} t+\phi_{2}\right)\right) \\
\phi(t) & =\phi_{0}+e^{-t / \tau_{C B O}} A_{3} \cos \left(\omega_{C B O} t+\phi_{3}\right)
\end{aligned}
$$

Eq. (4.25) can be used to study the CBO effect on the fitted muon $g-2$ frequency. An example is given in Fig. 4.6, where the data was generated with Eq. (4.25) but the fit was 


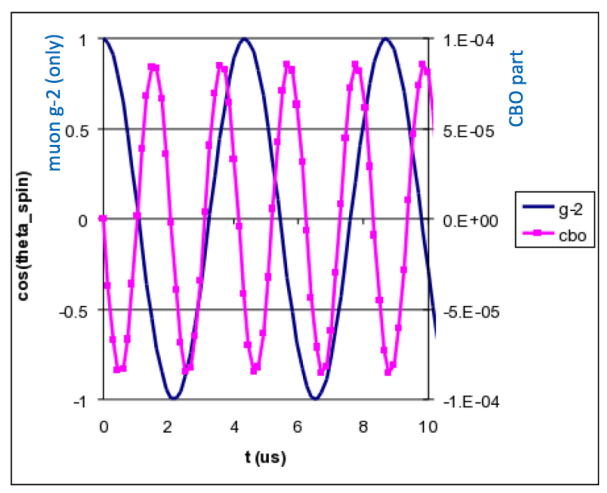

Figure 4.5: Spin precession from muon $g-2$ only and the additional spin precession with the full CBO [12].

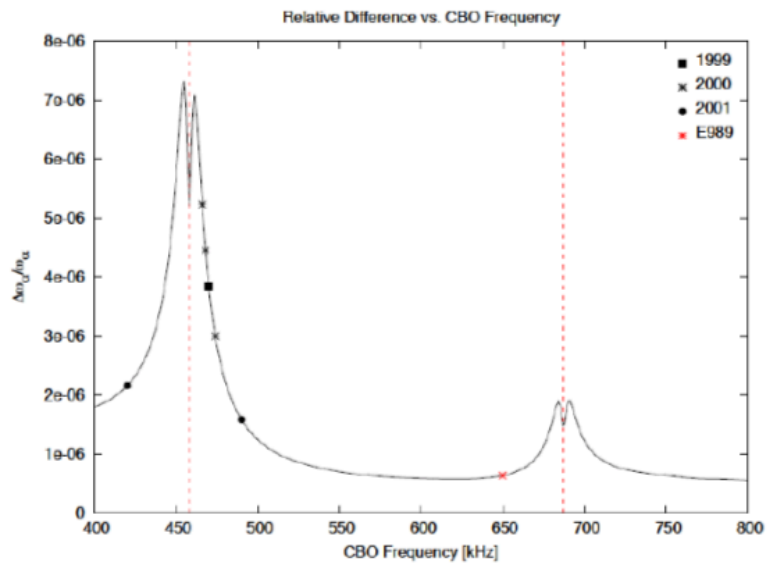

Figure 4.6: The effect of the CBO on fitted muon $g-2$ frequency vs. CBO frequency.

done with only the five parameter function [12]. The CBO systematic error comes primarily from uncertainties in the CBO's frequency and the functional form. For E989, the goal is to maintain the $\mathrm{CBO}$ systematic error under a $30 \mathrm{ppb}$ level.

\subsubsection{Lost Muons}

Lost muons can affect the measured average spin precession frequency. A lost muon systematic error occurs when muons lost from the storage ring at late times have a different average spin direction compared to the stored muons. In Eq. (4.25), we include a muon loss function $\Lambda(t)$ to describe the lost muons with respect to time. In fact, most of the injected muons will be lost because the storage ring has an injection capture efficiency of $\sim 5 \%$. Our goal is to reduce the lost muon rate after the injection by at least an order of magnitude as compared to the E821 lost muon rate.

After injection, most of the muons that follow orbits, which eventually intersect either collimators or other materials near the storage aperture, are removed during the scraping 


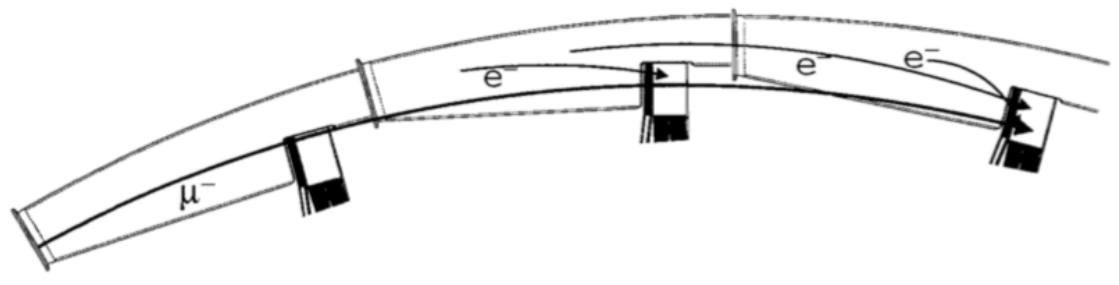

Figure 4.7: Diagram illustrating the detection of a lost muon $\left(\mu^{ \pm}\right)$by finding two-fold or three-fold coincidences between consecutive detectors.

period. But the surviving muons get perturbations from the electromagnetic fields inside the ring. Some of them still get lost due to the irreducible perturbations. This can be exacerbated by the betatron oscillations, especially when resonances occur. Muon losses can be studied by looking for two-fold or three-fold coincidences in consecutive detectors. The systematic error of lost muons can be estimated by using the muon loss function $\Lambda(t)$ in Eq. (4.25).

\subsubsection{E-field and Pitch Corrections}

The electrostatic quadrupoles introduce the electric field term in the expression for $\omega_{a}$. For a muon with the "magic momentum", that term vanishes. However, muons are injected into the storage ring as a bunch and they have a radial distribution, which means that not all of the muons sit on the "magic momentum". Moreover, the detector acceptance depends on the radial position of the muon when it decays, so that any coherent radial beam motion will amplitude modulate the decay positron distribution. Therefore, the systematic error due to the electric field must be understood. This requires a precise knowledge of the momentum distribution of the stored muons, which can be obtained by the so-called "fast rotation analysis" discussed in Chapter 5 .

If the momentum of stored muons is not perpendicular to the magnetic field, $\vec{\beta} \cdot \vec{B} \neq 0$ and the pitch correction should be considered as discussed in Chapter 2. To understand the pitch correction, we need to know the beam profiles, which can be monitored using the Straw Tracker system and Fiber Harp system. For E989, our goal is to have $<30$ ppb errors for the combined electric field and pitch corrections.

\subsection{D E-field from OPERA-3D}

In the Fermilab Muon $g-2$ Experiment, four Electrostatic Focusing Quadrupoles (ESQ) will provide the vertical focusing of the muon beam. Errors in the electric field of the quadrupoles will give rise to periodic forces which perturb the muon orbits. If the muon does not maintain the "magic momentum", there will be a correction to the measured muon moment anomaly due to the electric field. Therefore, we need to determine the electric field map to estimate such a correction as well as to understand the beam dynamics. A 3D 
electric field map will be much closer to reality than a 2D map. In this section, we will show the geometry for analyzing the electric field and present the 3D map obtained by using OPERA-3D [84].

In the experiment, the curved ESQ plates are not perfectly aligned. Their geometry, plus the cage (including trolley rails) and vacuum chamber, will introduce some higher order multipoles besides a pure quadrupole. The multipoles of electric fields in the muon storage ring were usually studied in a two dimensional approximation $[68,69,85]$. They can be expressed as

$$
V=\sum_{n=0}^{\infty} r^{n}\left[b_{n} \cos (n \theta)+a_{n} \sin (n \theta)\right]
$$

where $V$ is the electrostatic potential, and $b_{n}$ and $a_{n}$ are the normal and skew multipoles. The order of a multipole is $2 n$. In the E821 Muon $g-2$ Experiment, OPERA-2D was used to analyze the 2D field multipoles [68]. We know that the ESQ plates are not perfectly aligned, especially the new assembly of the very thin Q1 Mylar outer plates [86]. In E989 Muon $g-2$ Experiment, we performed the electric field analysis with several non-perfect models by using OPERA-2D to check and estimate our alignment, shown in Fig. 4.8.
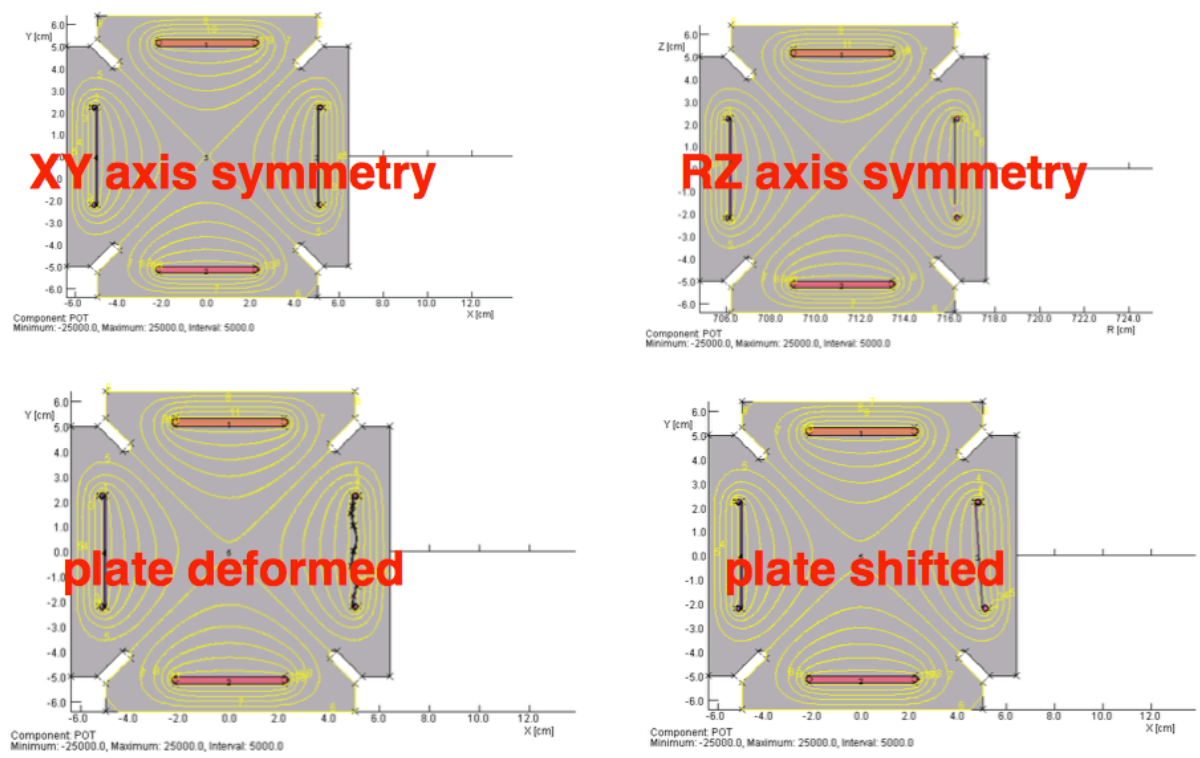

Figure 4.8: Electric field models from OPERA-2D: 1) XY axis symmetry: straight quadrupole plates; 2) RZ axis symmetry: curved quadrupole plates; 3) plate deformed: Q1 outer plates; 4) plate shifted: misalignment.

However, the 2D electric field analysis does not include the detailed geometry of the cage and vacuum chamber walls. Moreover, it cannot include the fringe fields at the two ends of each ESQ plate and at the inner side region of the vacuum chamber that the decay positron passes through. To achieve the goal of a high precision measurement of $a_{\mu}$, it is necessary to consider the 3D electric field map.

The curved multipoles are discussed in Ref. [87]. The main point here about the curved 
multipoles is how to solve Maxwell's equation or Laplace's equation with the geometry boundary situation we have. For example, Laplace's equation can be written as

$$
\nabla^{2} V=\frac{1}{1+x / r} \frac{\partial}{\partial x}\left[(1+x / r) \frac{\partial V}{\partial x}\right]+\frac{\partial^{2} V}{\partial y^{2}}+\frac{1}{1+x / r} \frac{\partial}{\partial z}\left(\frac{1}{1+x / r} \frac{\partial V}{\partial z}\right)=0
$$

The solution of Eq. (4.28) under some assumptions is discussed in Ref. [87], where expressions of the normal and skew multipoles are given.

In E989, the 3D electric field is studied by using OPERA-3D and 3D BEM. The latter is carried out by a group from Michigan State University, where they didn't consider the edges details of the ESQ plates and cage. However, their results do agree with ours here [88]. In the following sections, we are going to focus on the field analysis with OPERA-3D, the details of which can be found at Ref. [89].

\subsubsection{Geometry for OPERA-3D Simulation}

The ESQ geometry used in OPERA-3D analysis, shown in Fig. 4.9, basically follows the E821 ESQ engineering design drawings. The differences are listed here:

- The leads at the upstream end for connection between ESQ plates and quad extension are excluded;

- The "C-shapes" cap at the downstream end of ESQ plates are excluded;

- The "cut-gaps" of trolley rails are excluded;

- The vacuum chamber is replaced by a simple cylindrical tube;

- The support frames of cage are excluded;

- Cage and vacuum chamber are longer than the ESQ plates, but their two ends have different lengths relative to the ESQ plate ends (plates degree: $0^{\circ} \sim 13^{\circ}$, cage and vacuum chamber degree: $\left.-5^{\circ} \sim 20^{\circ}\right)^{1}$.

We consider a model with short ESQ plates only. The longer case can be extended in the way we deal with our data (see Sec. 4.3.2). To study the change of the field map due to the ESQ plate misalignment, we just consider a model with ESQ plates shifted, i.e., the outer plate is away from its design position by $0.5 \mathrm{~mm}$. It is possible to use the laser scan data discussed in Ref. [86] to build a better geometry ${ }^{2}$, however, it is very challenging. One may also fit the real geometry boundary to Maxwell's equation to solve for the fields, which is also very challenging since our ring geometries are very complex.

\footnotetext{
${ }^{1}$ Originally, we wanted to see the difference. However, the difference between the two ends is very small.

${ }^{2} \mathrm{~A}$ private discussion with a technician from the OPERA-3D field team.
} 


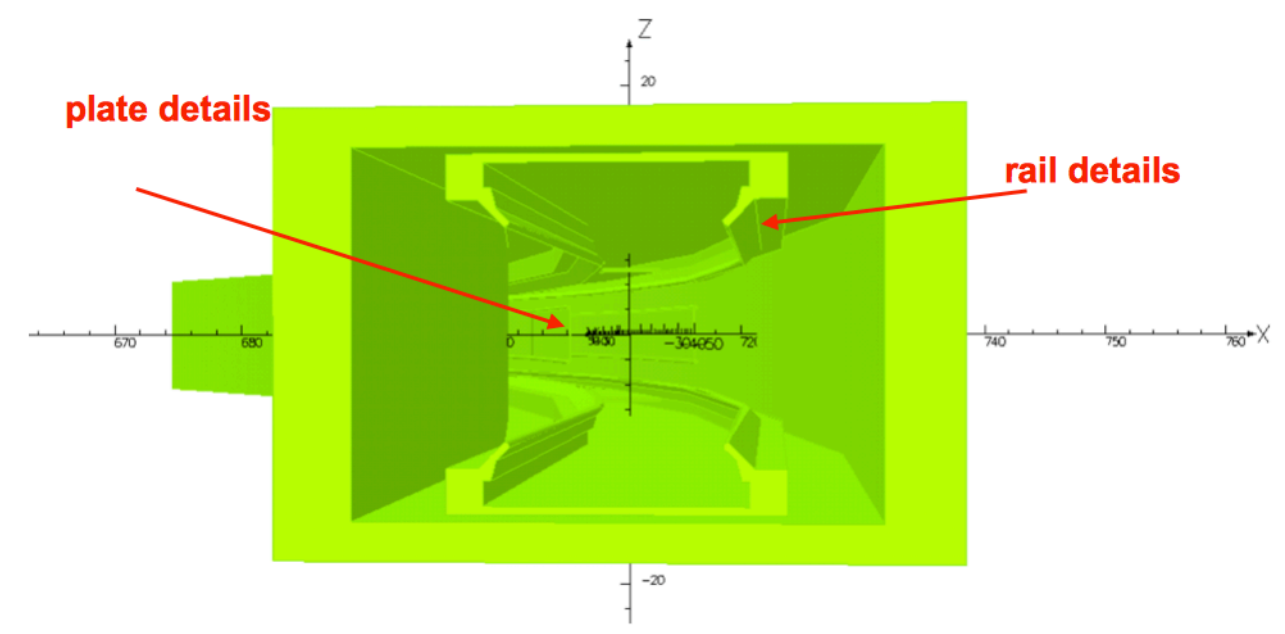

Figure 4.9: OPERA-3D geometry model: "short" plates inside a "long" cage and chamber.

\subsubsection{The 3D Electric Field Map}

The analysis was done by using OPERA-3D on a dedicated windows server ${ }^{3}$ which is also used to store the analysis data. One can also find all the analysis data on that machine. The cage and vacuum chambers are grounded. The quad plates have a potential of \pm 27.2 $\mathrm{kV}$ ("+" for upper and lower plates; "-" for inner and outer plates). An example of the electric potentials at arc with different radius is shown in Fig. 4.10, from which we can tell the fringe field effects at the two ends of the plates. Fig. 4.10 also gives us some hints to organize our data. The fringe field effects occur in a region of $< \pm 1^{\circ}$ relative to the plate end. This can be further demonstrated in Fig. 4.11, where the potential data at a cylinder surface with a "magic" radius is showed and is divided into three parts. The data table is under a cylindrical coordinate system. The following elements are contained: $\left(r, \theta, z, E_{r}\right.$, $\left.E_{\theta}, E_{z}, E, V\right)$.

We only consider a potential voltage $V=27.2 \mathrm{kV}$ since other situations can be calculated by rescaling the potential. The analysis contains three cases:

1. All ESQ plates locate at design position with top and bottom plates at potential $V$ and inner and outer plate have potential $-V$;

2. All ESQ plates locate at design position with only one of the four plates at potential $V$ and the other plates are grounded (The combination of four rescaled data sets can be used for scraping study);

3. One of four ESQ plates shifted from the design position but the other three plates maintain the design position (This is used for comparison with the ESQ plate misalignment case).

d The data sets of the above three cases are organized the same. They are in cylindrical coordinates and are divided into three parts: downstream end $\left(-1^{\circ} \sim 1^{\circ}\right)$, middle part

\footnotetext{
${ }^{3}$ Northwestern machine
} 


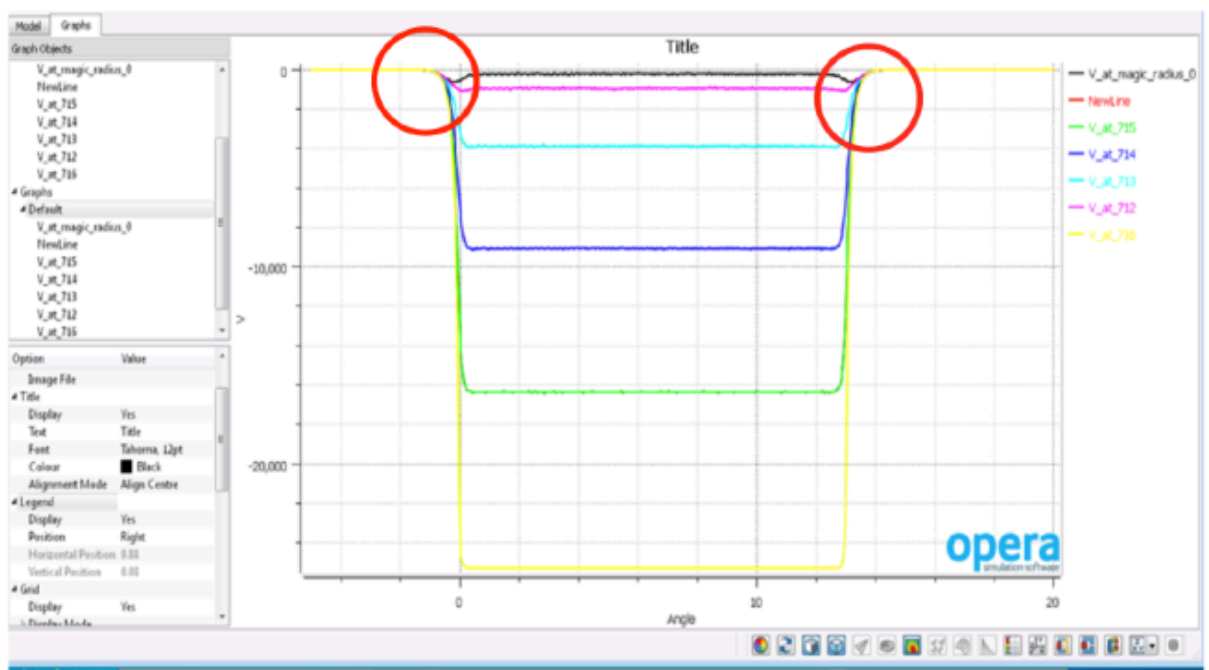

Figure 4.10: Electric potential at arc with different radius.

\section{Our field data on cylinder grid: Data is very large (>10 GB)}

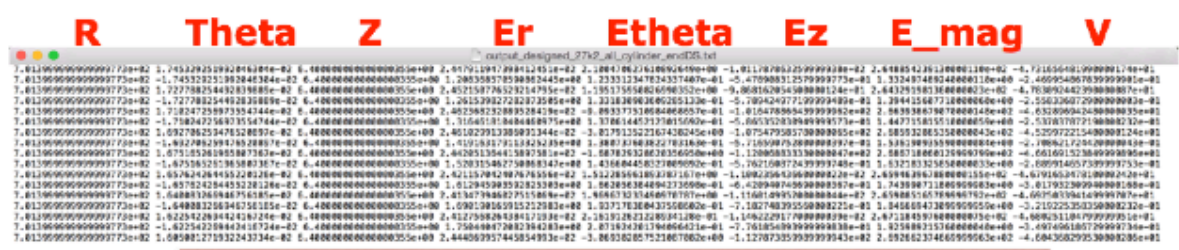

How are we going to use or deal with the data?

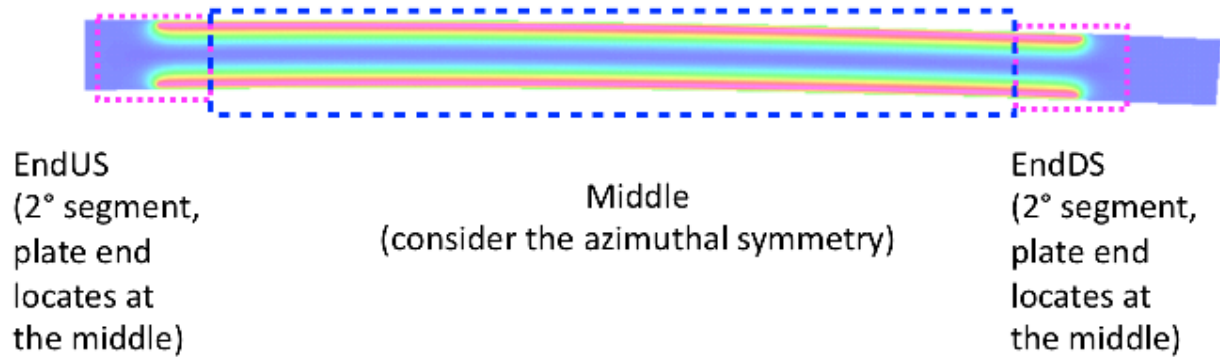

Figure 4.11: Potential at a cylinder surface with "magic" radius and data table. 
$\left(1^{\circ} \sim 12^{\circ}\right)$ and upstream end $\left(12^{\circ} \sim 14^{\circ}\right)$. The middle part data set is very large. Therefore, we consider the azimuthal symmetry for the middle part and average over $1^{\circ}$ to $12^{\circ}$, then obtain a cross-plane to stand for any middle section, shown in Fig. 4.12. For the case with long ESQ plates, we can use the same cross-plane to present the middle part map.

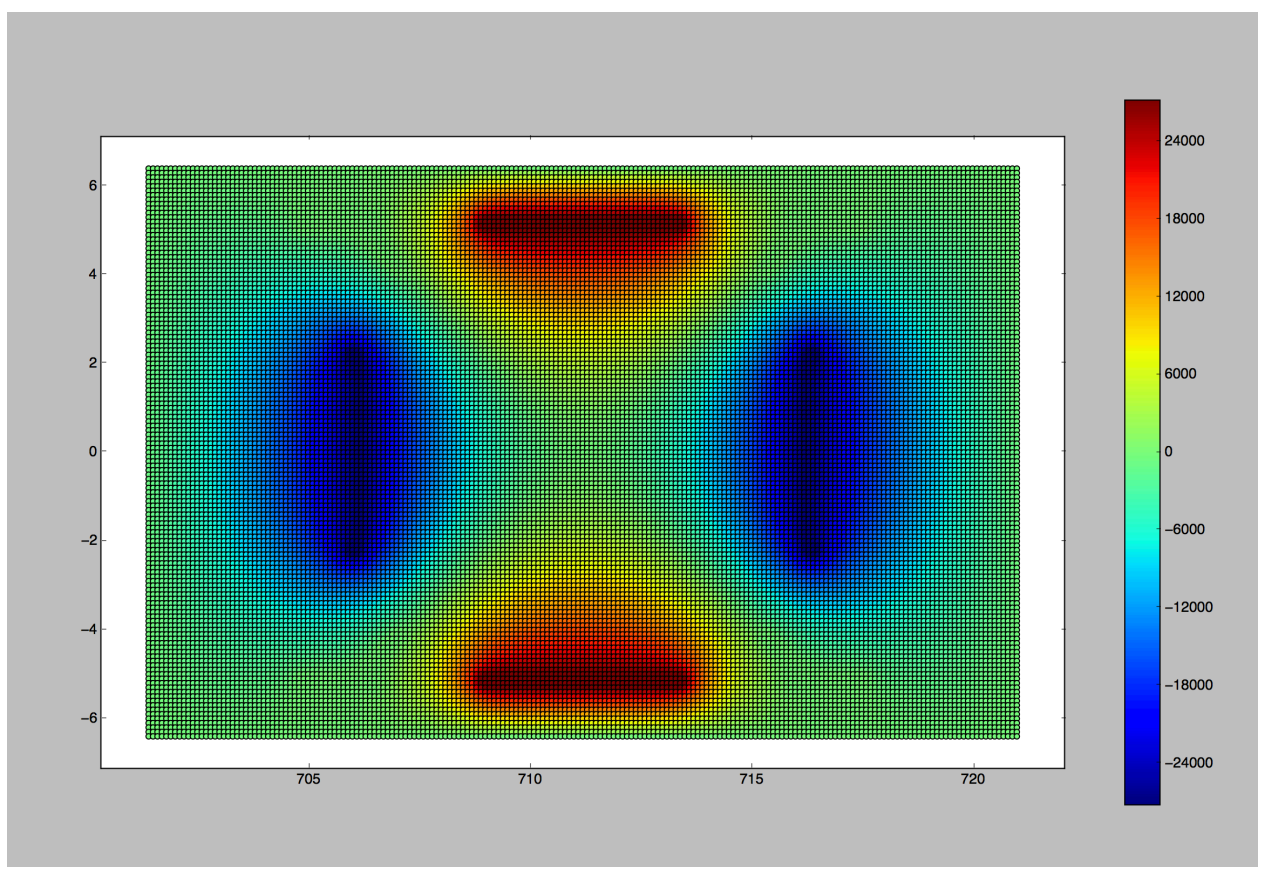

Figure 4.12: Electric equipotential map of quadrupoles from OPERA-3D with $\pm 27.2 \mathrm{kV}$ on the quadrupole plates and azimuthal average.

The case of only one quad plate charged and the other three plates grounded is useful for our scraping study where the potentials on four quad plates are asymmetric. The combination of four sub-datasets under this case can allow one to do any possible scraping study. An example of the field map of this type is shown in Fig. 4.13.

The test of the 3D electric field data obtained from OPERA-3D has been developed [90]. The 3D field data is now used by several groups to simulate and analyze the muon beam dynamics inside the storage ring. It will allow us to understand the beam dynamics more accurately and help us to achieve our E989 high precision measurement goal.

\subsection{Beam Resonances}

In a storage ring, the betatron tune is defined as the number of betatron oscillations for the beam traveling one turn around the ring:

$$
\nu_{x, y}=\frac{\phi_{x, y}(L)}{2 \pi}=\frac{1}{2 \pi} \oint \frac{d s}{\beta_{x, y}(s)}
$$

where $\beta_{x, y}(s)$ is the betatron amplitudes which vary over the length of the ring and $L$ is the circumference of the ring. The betatron tunes need to remain constant during ring operation. 

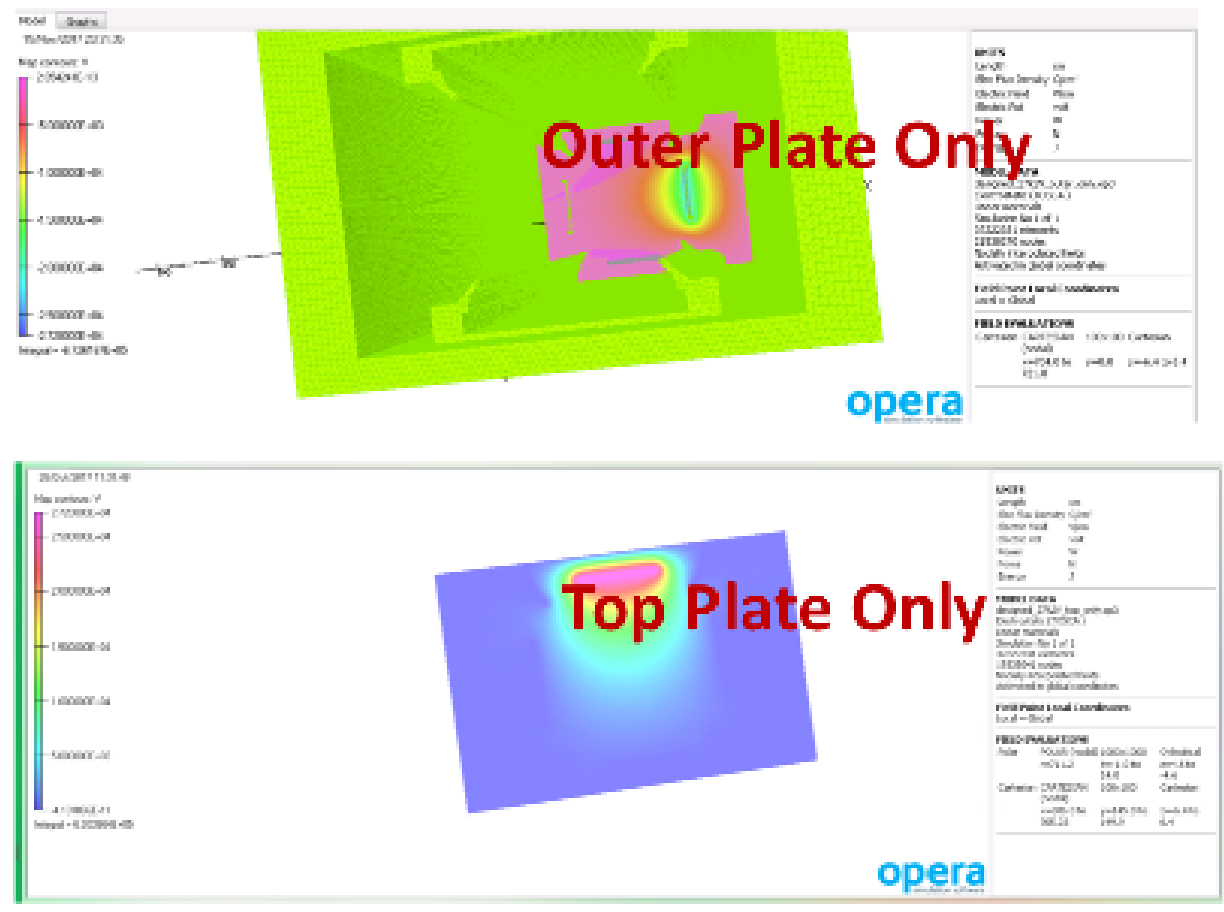

Figure 4.13: Example of the field map with one plate charged and the other three plates grounded.

Otherwise, the betatron tunes may drift and cause muon loss via resonance processes. When the operating tune point is placed on or close to a resonance line, the beam trajectories in phase space can be distorted and the beam becomes unstable which eventually will be lost or scraped by the ring aperture. This will affects the beam's survival and injection efficiency.

The betatron tunes depend on the beam optics that are mainly governed by the focusing field strength. For the muon storage ring using ESQ for the weak vertical focusing, the field index $n$ with dipole magnetic field $B$ and electric gradient $\partial E_{R} / \partial R$ is defined as

$$
n(s)=\frac{R}{\beta B} \frac{\partial E_{R}(s)}{\partial R}, \quad \beta=v / c
$$

where $s$ is the longitudinal coordinate with $\theta=s / R$ as the azimuthal angle. The muon equations of motion and their solution are discussed in Section 4.1. As an approximation, the horizontal and vertical tunes can be given by [91]

$$
\nu_{x}=\sqrt{1-n} \quad \text { and } \quad \nu_{y}=\sqrt{n}
$$

\subsubsection{Principles}

The principles of the beam resonances can be deduced firsthand from the muon's equations of motion. Some related work can be found in Ref. [69, 92, 68]. Below we just provide some general discussion. 
Let us start with an electric multipole with potential

$$
\Phi=(x+i y)^{k} \cos (N \phi)
$$

where $\phi$ is the azimuth around the ring and $\Phi$ represents the $2 k$-pole term and $N$ th azimuthal harmonic. The electric field can be solved by using $\vec{E}=-\nabla \Phi$. For one term of $\Phi$ of the form $\Phi_{k}^{m}=i^{m} C_{k}^{m} x^{l} y^{m} \cos N \phi$, we have

$$
\begin{gathered}
E_{x}=-\frac{\partial \Phi_{k}^{m}}{\partial x}=-i^{m} l C_{k}^{m} x^{l-1} y^{m} \cos N \phi \\
E_{y}=-\frac{\partial \Phi_{k}^{m}}{\partial y}=-i^{m} m C_{k}^{m} x^{l} y^{m-1} \cos N \phi
\end{gathered}
$$

Here, $C_{k}^{m}$ is the binomial coefficient in the expansion of $(x+i y)^{k}$, and hence $l+m=k$. Suppose the unperturbed betatron oscillation amplitudes are given by $x=H \cos \nu_{x} \phi$ and $y=V \cos \nu_{y} \phi$, then for the vertical electric field $E_{y}$, we have

$$
E_{y}=-i^{m} m C_{k}^{m} H^{l} V^{m-1} \cos ^{l} \nu_{x} \phi \cos ^{m-1} \nu_{y} \phi \cos N \phi
$$

Using the formulas in Ref. [69], we can rewrite Eq. (4.35) in the form of

$$
E_{y}=-i^{m} m C_{k}^{m} H^{l} V^{m-1} \frac{1}{2^{k-1}} \sum_{p=0}^{l-1} \sum_{q=0}^{m-2} \cos \left[(l-2 p) \nu_{x} \pm(m-1-2 q) \nu_{y} \pm N\right] \phi
$$

For such a driving term to the muon's betatron oscillation, the resonance occurs if

$$
(l-2 p) \nu_{x} \pm(m-1-2 q) \nu_{y} \pm N= \pm \nu_{y}
$$

Because $l, m, p, q$ and $N$ are just integers, we can simplify the above condition with

$$
L \nu_{x}+M \nu_{y}=N
$$

where $L, M$ and $N$ are integers. For the horizontal field $E_{x}$, we can apply the same analysis and the same condition will be obtained. One thing that should be noted is $\phi$ can also drive other resonances, but Eq. (4.38) specifies the highest order resonances.

For a weak focusing ring, the operating tune point satisfies the condition

$$
\nu_{x}^{2}+\nu_{y}^{2}=1
$$

Eq. (4.38) and Eq. (4.39) are normally plotted in a tune plane and show whether our ring operating points sit on any of the main resonances.

\subsection{2 g-2 Beam Resonances}

For our muon storage ring, we know that the ESQ plates are not perfectly aligned and the magnetic dipole field is not ideally uniform. Therefore, we want to see the strength of 
the resonance if the operating point is close to a resonance. In such a case, we start with a pure quadrupole with potential $\Phi=\alpha_{2} y^{2}$, where $\alpha_{2}$ is the coefficient. Then we have $E_{y}=-\partial \Phi / \partial y=-2 \alpha_{2} y$ and the equation of motion $\ddot{y}+\nu_{y}^{2} y=0$. For a $2 k$-multipole driving term of the form $\Phi_{k}^{m}=\alpha_{k} C_{k}^{m} x^{l} y^{m} \cos N \phi$, we have $E_{y}=-m \alpha_{k} C_{k}^{m} x^{l} y^{m-1} \cos N \phi$. If the operating point is slightly off resonance, then the tune drift is given by [69]

$$
\Delta \nu=\nu-\nu_{y}=\frac{\Delta n}{2}\left[\frac{l}{\nu_{x}}-\frac{m}{\nu_{y}}\right]
$$

Let $y=V \cos \nu_{y} \phi+f$ and $f$ here stands for the perturbation part, then we have

$$
\ddot{f}+\nu_{y}^{2} f=-\left(\nu_{y}^{2} / 2 \alpha_{2} 2^{-(k-1)} m \alpha_{k} C_{k}^{m}\right) H^{l} V^{m-1} \cos \nu \phi
$$

The solution of the perturbation $f$ is

$$
f=\frac{\left(\nu_{y}^{2} / 2 \alpha_{2} 2^{-(k-1)} m \alpha_{k} C_{k}^{m}\right) H^{l} V^{m-1} \cos \nu \phi}{\nu^{2}-\nu_{y}^{2}}
$$

Using Eq. (4.40), we can write the perturbation part as amount of the modulation:

$$
M=\frac{f}{V}=m C_{k}^{m} 2^{-(k+1)} \frac{\nu_{y}}{\Delta \nu} \frac{H^{l} V^{m-2}}{A^{k-2}} \frac{\Phi_{k}}{\Phi_{2}}
$$

Here, $A=45 \mathrm{~mm}$ is the radius of our storage aperture and the result is compared to the pure quadrupole potential $\Phi_{2}$. Now, if we require that the modulation for each multipole acting alone should be less than one percent of the vertical amplitude, this can tell us the maximum amount of each multipole that can be tolerated. For example, for an operating point with $n=0.148$ and a 20th-pole with $l=6, m=-4$ and $N=4$, we have $\Phi_{10} / \Phi_{2} \sim 7 \%$. The fractional increase of the vertical amplitude in one turn of the ring is $2 \pi \Delta \nu M$.

Usually, in the presence of a small gradient perturbation $k(s)$, the tune will be drift by $[70]$

$$
\delta \nu=\frac{1}{4 \pi} \int k(s) \beta(s) d s
$$

As an approximation, we may use the semi-empirical formulas for the tunes [92]

$$
\begin{aligned}
& \nu_{x}=\sqrt{1-n}+\Delta \nu_{x}(n) ; \quad \Delta \nu_{x}(n)=\frac{0.0452 n^{2}}{\sin \left(\frac{\pi}{2} \sqrt{1-n}\right)} \pm 0.000006 \\
& \nu_{y}=\sqrt{n}+\Delta \nu_{y}(n) ; \quad \Delta \nu_{y}(n)=\frac{0.0512 n^{2}}{\sin \left(\frac{\pi}{2} \sqrt{n}\right)} \pm 0.000006
\end{aligned}
$$

In our storage ring, the four quadrupoles altogether occupy about $43.3 \%$ of the ring. The period of the ring lattice $(\pi R / 2)$ is much less than the period of horizontal betatron oscillation which is close to $2 \pi R$, and especially less than that of vertical betatron oscillation $(\sim 6 \pi R)$. Moreover, the operating point $n$ we choose is very small, i.e., the vertical focusing 
and the reduction of horizontal focusing are very weak. For these reasons, the field index $n$ is averaged over the orbit, $n=\langle n(s)\rangle=0.433 n_{0}$, in a "smooth approximation" with very good accuracy (here, $n_{0}$ is the field index inside the quadrupole). In reality, because of the fringe field effects at the ends of ESQ plates, the "effective length" of the quadrupoles may be a little larger than the true quad plate length and therefore, can also affect the tune shift. The beam simulation with the 3D electric field map will help us understand such an "effective length" effect. But this effect should be less than $2 \%$ as we can tell from Fig. 4.10. In fact, we could make a rough guess here. From Fig. 4.10, we see the "effective part" of one end is about $0.3^{\circ}$. For one quadrupole region, there is a short quad part and a long quad part. Therefore, the "effective part" in total is about $0.2^{\circ} \times 4 / 90^{\circ} \simeq 0.0089$. If this is true, the field index will also change, i.e., $n=\langle n(s)\rangle=0.4389$. Of course, we need a beam simulation with the 3D electric field to verify this [93].

The field index $n$ increases for higher quadrupole voltage. The operating quadrupole voltage should be chosen to avoid the beam resonances, where a weak-focusing storage ring operates using the approximate condition in Eq. (4.39). Therefore, the operating point should not intersect any of the primary resonance lines shown in Fig. 4.14.

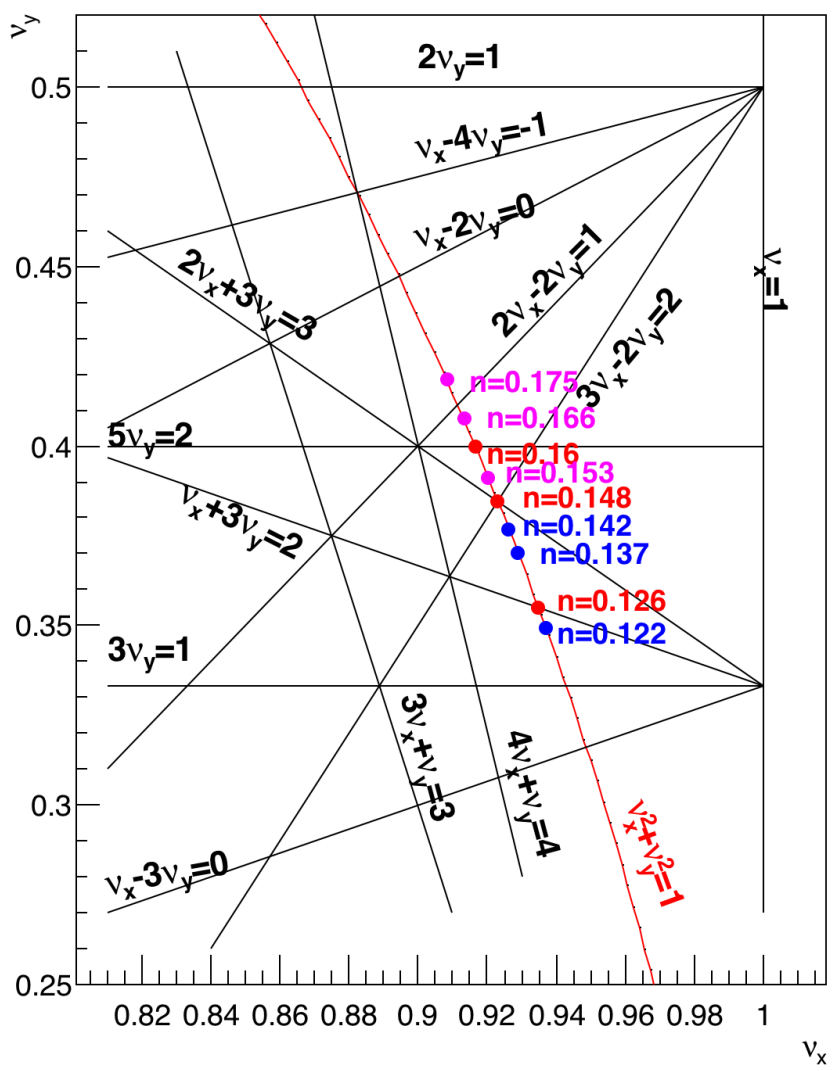

Figure 4.14: The tune plane with some resonance lines: E821 ran at $n=0.122,0.137$ and 0.142 , and the possible $n$ values for E989 are 0.142, 0.153, 0.166, 0.175 [91].

During the commissioning, we also perform a quad resonance scan to understand the beam resonances. Fig. 4.15 shows a quad resonance scan to test our low-end run values of around $14.4 \mathrm{kV} / 20.2 \mathrm{kV}$, where we can tell there are some interesting kinks at around 19.0 
$\mathrm{kV}$ and $21.2 \mathrm{kV}$. A fine-grained quad scan can help us locate the betatron resonances, as is shown in Fig. 4.16 [94] and Fig. 4.17. We can clearly identify there exist resonances at around $16.7 \mathrm{kV}, 18.8 \mathrm{kV}$ and $21.2 \mathrm{kV}$.

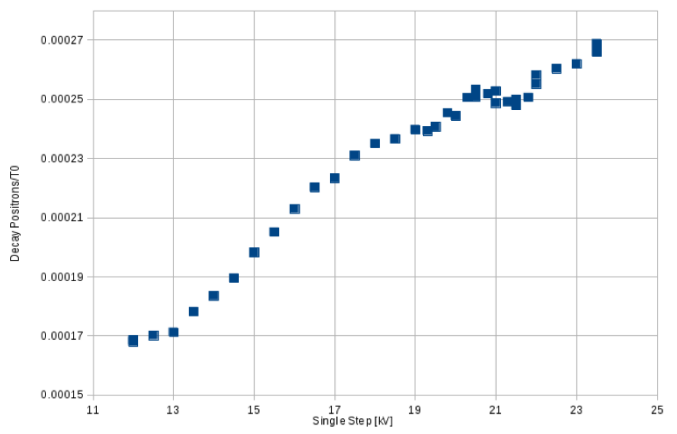

Figure 4.15: Example: quad resonance scan (Stored muons are represented by "Decay Positrons/T0") [95].

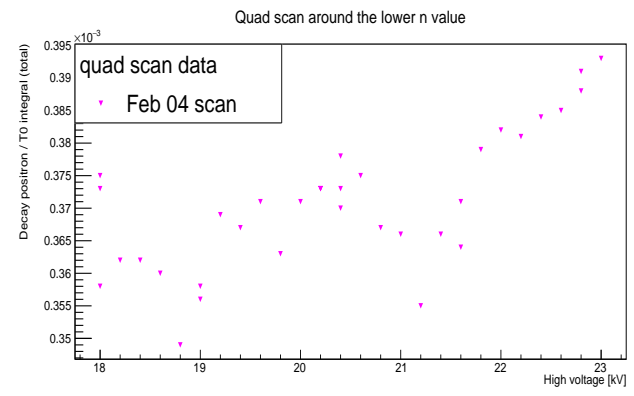

Figure 4.16: Example of fine-grained quad resonance scan (without scraping)-stored muons: represented by "Decay positrons/T0".

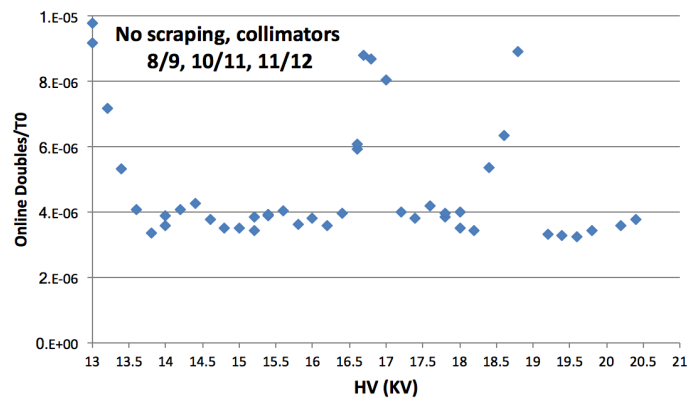

Figure 4.17: Example of fine-grained quad resonance scan (without scraping)-lost muons: represented by "Online Doubles/T0".

For E989, the beam dynamics becomes extremely important to reach a high precision measurement. The beam resonances, as well as muon losses, CBO, E-field and pitch corrections, and other beam dynamics uncertainties, need to be understood and studied carefully. The 3D electric field map will be very useful to simulate the beam dynamics and confirm the results we obtain from the experimental data. 


\section{CHAPTER 5}

\section{FAST ROTATION ANALYSIS}

In this chapter, we will focus on the so-called fast rotation analysis. We will show how to use the beam de-bunching information to extract the muon radial momentum distribution, which is the key input for the electrical field correction to $g-2$.

\subsection{Motivation and Ideas}

In the muon storage ring, particles are injected into the storage ring as a bunch. The bunch has a radial distribution over the whole aperture. Since all the particles in the bunch travel at almost the speed of light, those at inner equilibrium radii will move steadily ahead of those at outer equilibrium radii, as is shown in Fig. 5.1. Thus the time distribution of particles on subsequent turns exhibits an ever increasing width directly related to their initial radial distribution.

Muons in a bunch with larger momentum $\left(p>p_{m}\right)$ will naturally assume higher orbits $\left(r>r_{m}\right)$, which take longer to complete one cyclotron revolution. After some time (around 100-1000 revolutions), a muon with lower momentum will lap a muon with high momentum in the same bunch and the bunch will stretch. With some appropriate assumptions, the bunch structure depends uniquely on the momentum distribution. The fast rotation analysis (FRA) is a method that uses a model of the time evolution of the bunch structure to determine the momentum distribution inversely (as well as the radius distribution).

The momentum distribution is an important element needed to analyze and estimate the uncertainties on the $g-2$ measurement. Therefore, the FRA is necessary and must be treated appropriately. We have two different methods to execute the FRA: a minimized $\chi^{2}$ method and a Fourier Transform method. We will give a brief introduction about the Fourier Transform method and focus on the minimized $\chi^{2}$ method to extract the momentum distribution for the experiment.

\subsection{Fourier Transform Method}

In the BNL $g-2$ experiment, the Fourier transform algorithm was introduced to analyze the muon momentum distribution as a complement to the $\chi^{2}$ minimization method used in both CERN III $g-2$ experiment and BNL $g-2$ experiment. The Fourier transform method calculates the cosine Fourier integral using data available for a given detector and the first 


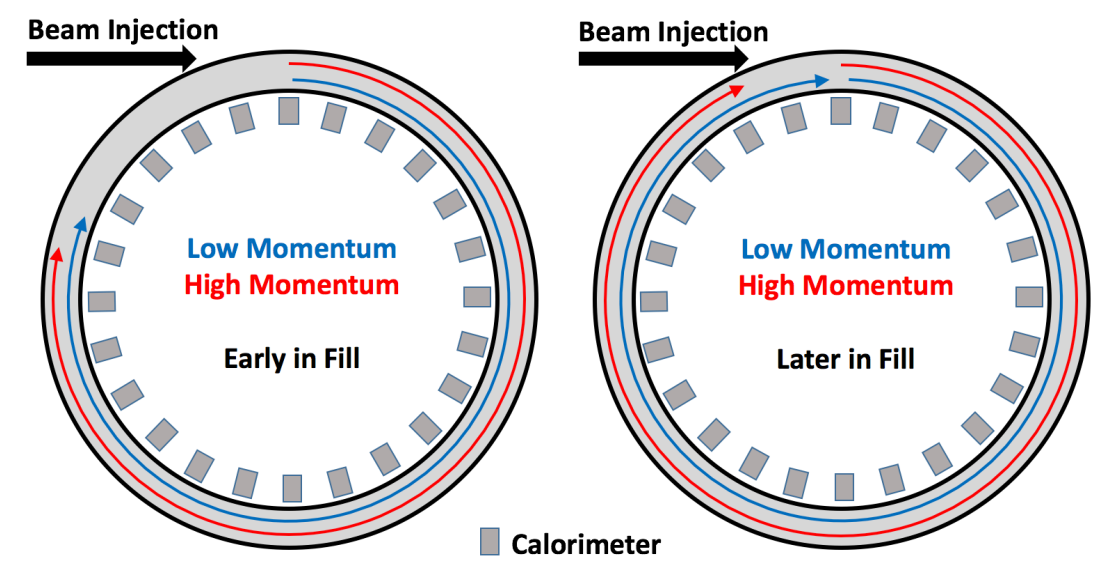

Figure 5.1: Cartoon of beam debunching for fast rotation analysis.

approximation for the initial time for the detector:

$$
\operatorname{Re} \Phi\left(f, t_{s} ; t_{m}\right)=\int_{t_{s}}^{t_{m}} F(t) \cos 2 \pi f\left(t-t_{0}\right) d t
$$

Both the $\chi^{2}$ minimization method and the Fourier transform algorithm deal with the fast rotation signal, which combines the decay electron (positron) signal observed by the 24 detectors and a fit function or ideal signal:

$$
F(t)=\frac{S(t)}{N e^{-t / \tau}\left[1+A \cos \left(\omega_{a} t+\phi\right)\right]}
$$

where $S(t)$ is the decay electron signal. An overview of the signals is shown in Fig. 5.2. However, in reality, we also need to consider the muon loss and CBO effects on the fast rotation signals. Ideally, we want to remove them from the decay electron (positron) signals.

The main challenges here include determining the initial time $t_{0}$ and the start time $t_{s}$, which can be well-treated as we deal with the fast rotation signal. In $g-2$, with reasonable assumptions we are able to calculate the real part of the Fourier transform of the fast rotation signal, which can represent the actual revolution frequency distribution, as is shown in Eq. (5.1). A comparison between the Fourier transform method and the $\chi^{2}$ minimization method for the BNL $g-2$ experiment is shown in Fig. 5.3. We will not extend the discussion about the Fourier transform algorithm here.

\section{$5.3 \quad \chi^{2}$ Minimization Method}

Particles are injected into the storage ring as a bunch. Therefore, the muons have a radial distribution over the whole aperture. The initially injected bunch has a narrow time width (i.e., $120 \mathrm{~ns})$, which is less than the revolution time $(\sim 149 \mathrm{~ns})$. Since all the particles in the bunch travel at almost the speed of light, those at inner equilibrium radii will move 

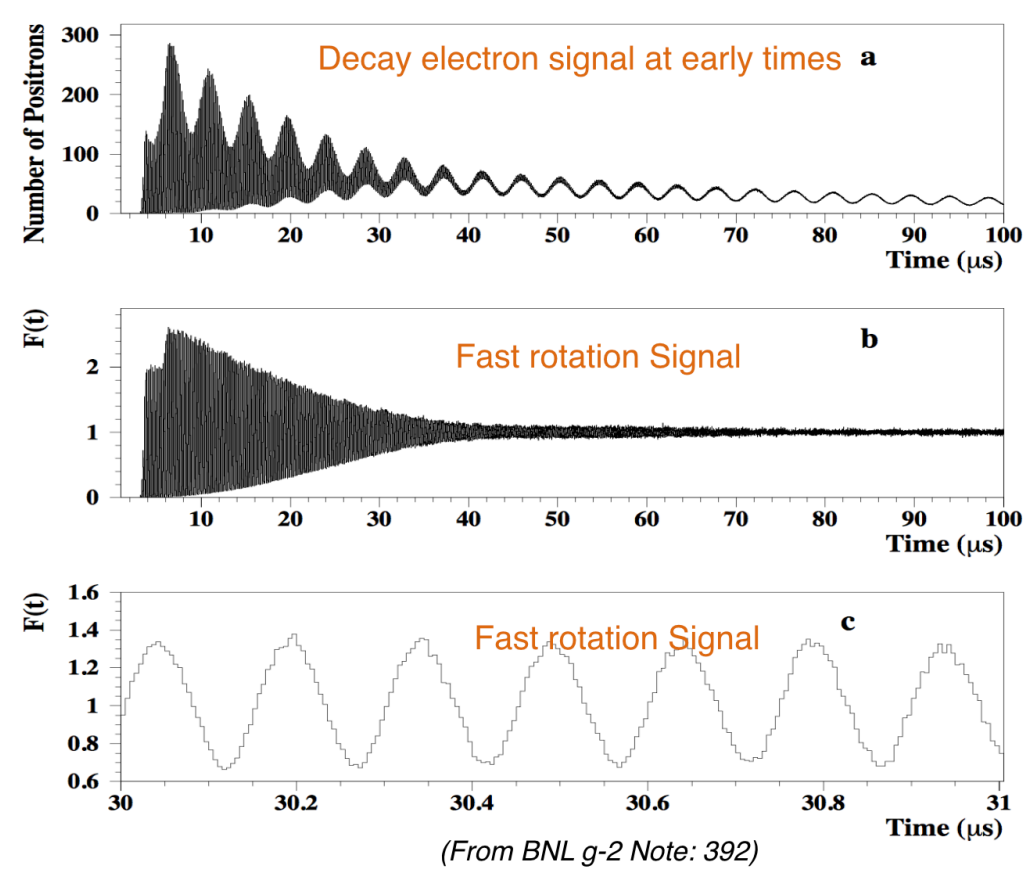

Figure 5.2: An overview of the fast rotation signals.

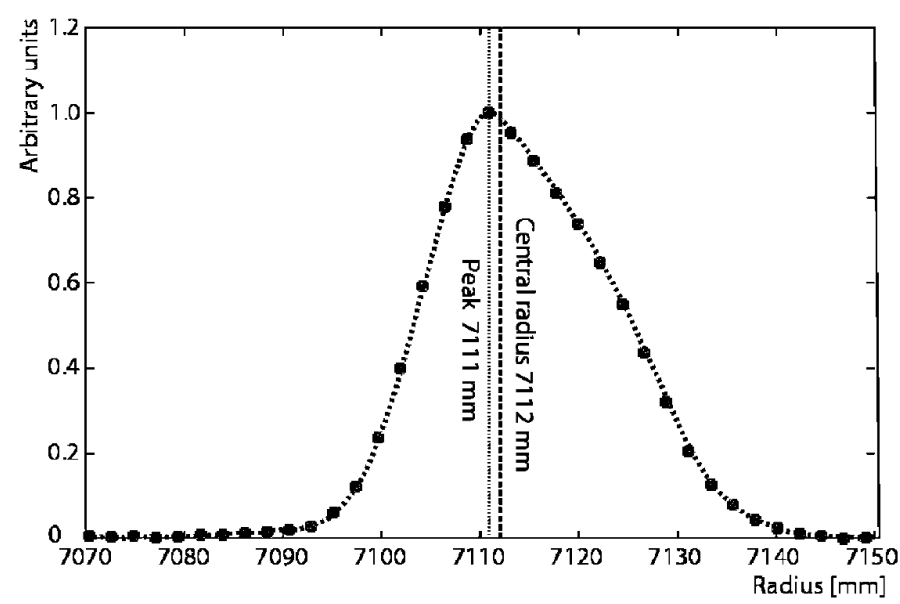

Figure 5.3: The distribution of equilibrium radii, as determined from the fast rotation analysis: the dashed curve is obtained from the modified Fourier analysis; and the solid circles are from $\chi^{2}$ minimization method [44]. 


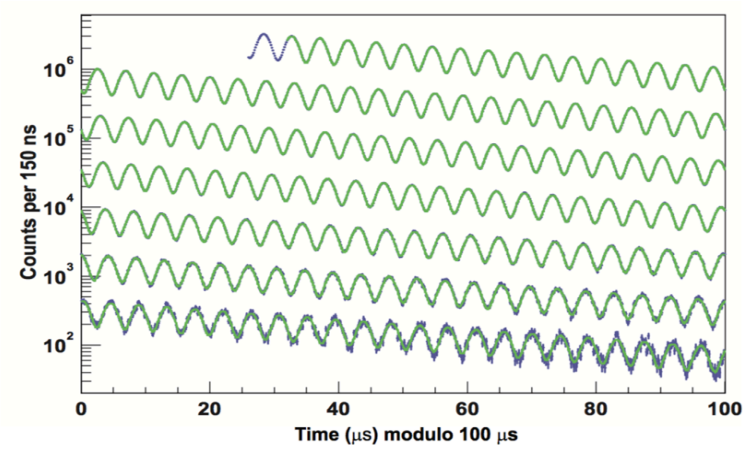

Figure 3.8: Histogram, modulo $100 \mu \mathrm{s}$, of the number of detected electrons above $1.8 \mathrm{GeV}$ for the 2001 data set as a function of time, summed over detectors, with a least-squares fit to the spectrum superimposed. Total number of electrons is $3.6 \times 10^{9}$. The data are in blue, the fit in green.

Figure 5.4: Example of decay positron count histogram [12].

steadily ahead of those at outer equilibrium radii. Thus the time distribution of particles on subsequent turns exhibits an ever increasing width directly related to their initial radial distribution. For the FRA, we use the combined decay positron count histogram in a single calorimeter as our input to the analysis, of which an example is shown in Fig. 5.4.

\subsubsection{FRA Assumptions}

The fast rotation analysis assumes that we know the injection pulse time shape, which is usually obtained from the upstream beamline as discussed in Section 2.3.1. In reality, the pulse shape we obtain from the upstream beamline is slightly different from that is injected into the storage ring. This is because the pulse shape is changed during transport.

The injection beam contains some positrons, along with the muons. When stored in the ring, the positrons will spiral in and get lost after several microseconds. Those positrons will also affect the injection pulse shape. Moreover, because the Kicker cannot perform perfectly at the first turn and the scraping process will remove some parts of the beam, we cannot just use the pulse shape obtained from upstream (i.e., the T0 shape) as our input for the fast rotation analysis, in practice. To obtain the pulse shape, we can try to rebuild it using the decay positron signals. With large statistics, we can assume that the rebuilt pulse shape can stand in for the injection pulse shape. This will be discussed in the following sections.

For the fast rotation analysis, we also need to know the beam injection time. Ideally, this can be deduced from the beam transport. However, for almost the same reason as for the injection pulse shape above, we have to find a reliable "relative" injection time for our fast rotation analysis. Here, the "relative" injection time means the time referring to our selected data and is not the time when the beam bunch is injected into the storage ring. The "relative" injection time should differ from the injection time by an integer number of revolution periods.

To find out the injection time or "relative" injection time, we try to fit the arrival time 
of each bunch by number of turns with a linear relation:

$$
t_{\text {bunch }}=N_{\text {turns }} \cdot T_{C}+t_{0}
$$

where $T_{C}$ is the bunch revolution time and $t_{0}$ is the ("relative") injection time. We will discuss the details in the rest of this chapter.

\subsubsection{Mathematical Details}

The time distributions of muons is divided into radial bins and from the known rotation time the relevant positions (bunch lengths) are calculated on subsequent turns. Some initial discussions about the idea and procedure are given by P. Hattersley and H. Jöstlein [96].

Suppose that the storage aperture is divided into $I$ radial (momentum) bins with the suffix $i$. Let the contents of the radial bins be $f_{i}$, which is the fraction of the beam oscillating around radial bin $i$ and what we want to solve for. What the detector (a single one) observes is the decayed positron count as a function of time. If the time bins of the histogram are labeled with the suffix $j$, the counts in bin $j$ can be denoted as $N_{j}$ (or $N(j)_{o b s}$ ), which is our input data.

The expected count at time bin $j$ is given by

$$
C_{j} \equiv N(j)_{\text {exp }}=\sum_{i=1}^{I} f_{i} \beta_{i j}
$$

where $\beta_{i j}$ describes how the bunch will evolve with both time and radius, and it is a geometrical factor that can be calculated separately for each combination of $i$ and $j$. In other words, $\beta_{i j}$ describes the contribution from the radial bin $i$ to the time bin $j$ and the expected contribution from radial bin $i$ to the count in time bin $j$ is just $f_{i} \beta_{i j}$.

The minimized $\chi^{2}$ method of fast rotation analysis is to give maximum agreement between the observed counts $\left(N_{j}\right)$ and the expected counts $\left(C_{j}\right)$. Here,

$$
\chi^{2}=\sum_{j} \frac{\left(N_{j}-C_{j}\right)^{2}}{Z_{j}}=\sum_{j} \frac{\left(N_{j}-\sum_{i} f_{i} \beta_{i j}\right)^{2}}{Z_{j}}
$$

The weighting factor $Z_{j}$ should be equal to the expected counts $C_{j}$ in bin $j$. However, the $C_{j}$ are initially unknown, so we replace them with the $N_{j}$ in the first pass. With the $f_{i}$ obtained we will carry out a second pass with

$$
Z_{j}=\sum_{i=1}^{I} f_{i} \beta_{i j}
$$

Some further iterations may be executed but they do not appreciably alter the results [96].

To minimize $\chi^{2}$ by adjusting the $f_{n}\left(n=1,2, \ldots, I, f_{n}\right.$ is a specific factor), we require 
that

$$
\frac{\partial \chi^{2}}{\partial f_{n}}=0
$$

for each $n$. By assuming that $Z_{j}$ are constant (this is not strictly true but a good approximation), we get

$$
\frac{\partial \chi^{2}}{\partial f_{n}}=\sum_{j} \frac{-2 \beta_{n j}\left(N_{j}-\sum_{i} f_{i} \beta_{i j}\right)}{Z_{j}}=0
$$

Therefore,

$$
\sum_{j} \frac{\beta_{n j} \sum_{i} f_{i} \beta_{i j}}{Z_{j}}=\sum_{j} \frac{\beta_{n j} N_{j}}{Z_{j}}
$$

Reordering of the summation gives

$$
\sum_{i} f_{i} \sum_{j} \frac{\beta_{i j} \beta_{n j}}{Z_{j}}=\sum_{j} \frac{N_{j} \beta_{n j}}{Z_{j}}
$$

The Eq. (5.10) represents a set of equations, one for each $n$. These equations can be solved iteratively and give the values of $f_{i}$. As we have discussed above, the procedure can be represented schematically as:

1. first pass: let $Z_{j}=N_{j}$, then obtain the $f_{i}$;

2. second pass: use the $f_{i}$ of the first pass and let $Z_{j}=\sum_{i} f_{i} \beta_{i j}$, then obtain the more accurate $f_{i}$.

\subsubsection{Calculation of $\beta_{i j}$}

The geometry factors $\beta_{i j}$ are known functions of the ring geometry and the apparent time structure of the injected bunch. In order to calculate the geometry factor $\beta_{i j}$, we need to know the time distribution of the injection pulse, which can be obtained from the upstream beamline or rebuilt by using the decay positron signal. In principle, the time distribution of the injection pulse can be any shape. In our simulation, we consider a Gaussian pulse and a "W-shape" pulse. BNL $g-2$ uses a Gaussian pulse with a width of around 25 ns; while for the Fermilab Muon $g-2$ Experiment, because the beam bunch is rotating in phase space in the Recycler, the projected time distribution of the pulse has some tails at each end and the whole pulse looks like a "W". The so-called "W-shape" pulse can be determined and measured from the upstream beam line.

According to the parameterized radial bins of the radial distribution, each radial bin is supposed to represent a symmetric triangular distribution falling to zero at the center of the adjacent bin, as shown in Fig. 5.5 [96]. The sum of those triangles gives a curve which appears to be a reasonable approximation to the real distribution. 


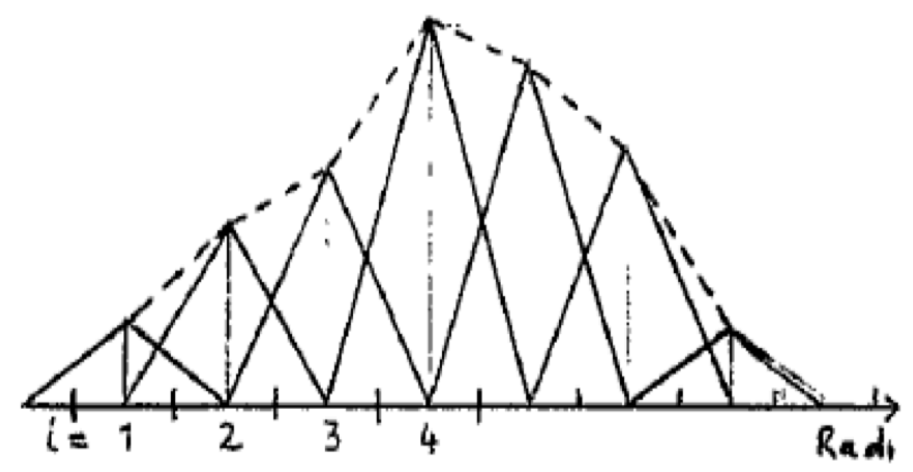

Figure 5.5: Parameterized radial bins of the radial distribution.

In order to calculate the geometry factors $\beta_{i j}$, we need to know the time distribution of the injection pulse. Here, we could just suppose that we have a square pulse of width $\Delta$. The real injection pulse shape can be treated using square pulses with some weighting factors.

Because of the beam de-bunching, the square pulse shape will be skew and the degree of the skewness is measured by the time length $\delta$, as shown in Fig. 5.6. The cut area at some time $t_{j}$ represents the contribution of radial bin $i$ to the time bin $j, \beta_{i j}$. Here, $j$ is determined by $x$, the distance in time between the cut and the geometrical center of the wedge. We can unify the contribution by define the maximum contribution as unity.

\section{Calculation of $\delta$ :}

Assume at beginning, the injection pulse is not skew $(\delta(t)=0)$ and all the particles move roughly at the same speed $v$. After some time $t$, we get a pulse shape shown in Fig. 5.6. Particles at the inner edge radial orbit should move approximately the same distance as particles at the outer edge radial orbit: $s=s_{1}=s_{2}=v t$. Therefore, in the unit of angle arc,

$$
\delta=\frac{1}{2}\left(\theta_{1}-\theta_{2}\right)=\frac{1}{2}\left(\frac{s_{1}}{R_{1}}-\frac{s_{2}}{R_{2}}\right)=\frac{1}{2} \frac{\left(R_{2}-R_{1}\right)}{R_{1} R_{2}} s=\frac{\Delta R}{2 R_{1} R_{2}} s
$$

Since $R_{1,2} \gg \Delta R$, we replace them by $R_{i}=\frac{1}{2}\left(R_{1}+R_{2}\right)$. Hence, $\delta=\frac{\Delta}{2 R_{i}^{2}} s$. In the unit of time, multiplying two sides by $\frac{R_{i}}{v}$, we have

$$
\delta(t)=\frac{\Delta R}{2 R_{i}} t
$$

\section{Geometry Factor for Fast Rotation Analysis:}

When we say a pulse, we mean the pulse in time-radius space. However, there should be another dimension to indicate the count probability of particles in this pulse, i.e., $P(t, r, p)$, 


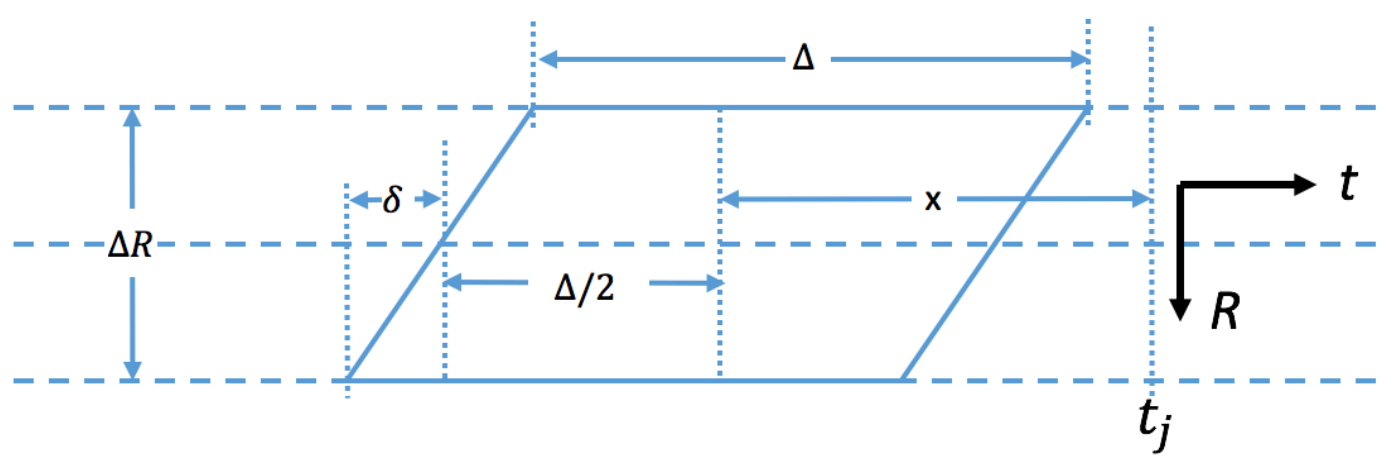

Figure 5.6: Calculation of $\delta$.

where $p$ is the momentum. If the momentum distribution is uniform $(d p / d t=0)$, then the probability can be replaced by $P(t, p)$ or $P(t, r)$.

For the fast rotation analysis, we can consider a square pulse cell or a Graziano pulse cell. For a square pulse cell, we can always assume that the momentum distribution is uniform over time. Therefore, we can use a changeable parallelogram to replace the square pulse cell for calculation the geometry factors, as is shown in Fig. 5.7. Based on the time
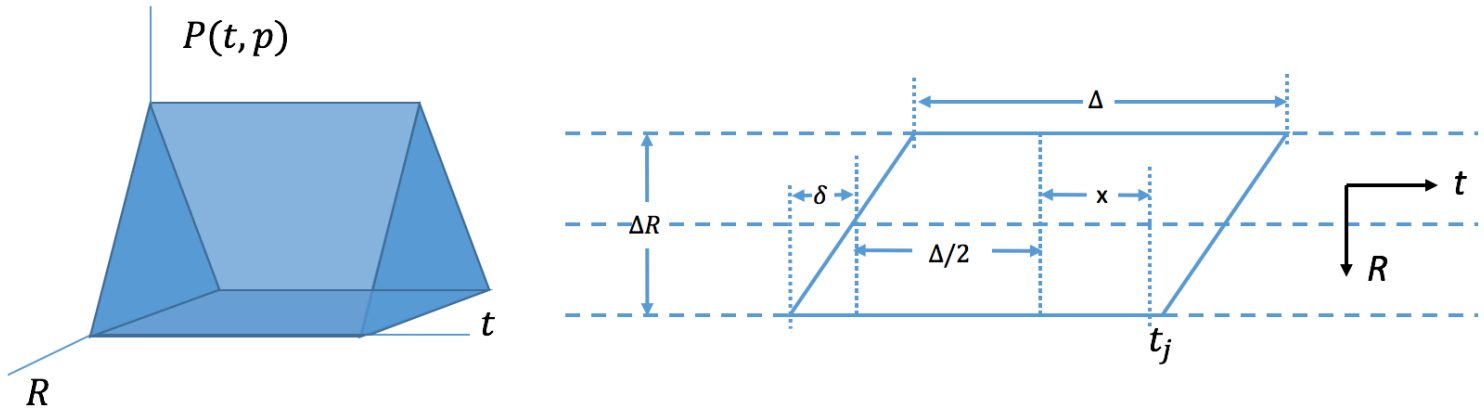

Figure 5.7: Square pulse cell.

cut positions, we can divide the skewness into several cases. The geometry factor is then calculated by using the cut area. For a Graziano pulse shown in Fig. 5.8, we can following the same procedure and divide the skewness into several cases. The details of the calculation of geometry factors for the square pulse and the Graziano pulse are given in Appendix C.

\subsubsection{Solve the Radial Bin Content $f_{i}$ by Matrix}

In this section, we discuss how to solve the radial bin content $f_{i}$ by using matrix knowledge. First the transposition of a matrix is given by

$$
A_{i j}=A_{j i}^{T}
$$




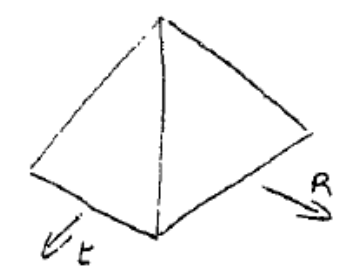

Figure 5.8: Example of Graziano pulse.

We consider a system of linear equations

$$
A x=b
$$

where $x$ is the unknown vector, $A$ is a matrix and $b$ is a vector. If $A$ is a square matrix that is nonsingular (its determinant is not zero), there is a unique solution $x=A^{-1} b$. In general, the "worst possible" way to compute the solution $x$ is to compute the matrix inverse. There are two central principles of numerical linear algebra: never compute a determinant and never compute the inverse of a matrix. The best solution methods for linear systems are based on Gaussian elimination or iteration.

The normal equation of the least square problem is given by

$$
A^{T} A x-A^{T} b=0
$$

Now considering if $A^{T} A$ is invertible (the columns of $A$ are linearly independent), then the above equation has a unique solution:

$$
x=\left(A^{T} A\right)^{-1} A^{T} b
$$

The quantity $\left(A^{T} A\right)^{-1} A^{T}$ is called the pseudo inverse of $A$. With the pseudo inverse, the linear regression problem can be solved.

In practice, the pseudo inverse is not computed directly. The normal equations are solved by elimination or an iterative method; or, better yet, the pseudo inverse is computed using the singular value decomposition of $A$. The reason for not simply solving the normal equations is that these equations may be ill-conditioned, for example the matrix $A^{T} A$ may be nearly singular (not invertible).

Our case is similar to the linear system in Eq. (5.14) after some mathematical steps as discussed in Section 5.3.2. Here, instead of fitting the decay positron histogram directly, we are working on a linear system to solve for the radial bin contents.

\subsubsection{Simulation}

To test the $\chi^{2}$ minimization method in the fast rotation analysis, we have a full simulation to generate the decay positron signal, analyze the data and compare with the expected data. We will discuss the details in this section. 


\section{Bunch Overlap Time:}

For fast rotation analysis, we usually care about the time of the bunch overlapping with itself. For muons cycling at an equilibrium radius $r$, the momentum $p_{r}$ is given by

$$
p_{r}=\operatorname{rcB} * 10^{-3}
$$

where $c=299.792458 \mathrm{~mm} / \mathrm{ns}$ is the speed of light, $B$ is the magnetic field in units of Tesla and momentum $p_{r}$ is in units of $\mathrm{MeV} / \mathrm{c}$. Inversely, we can obtain the revolution radius if we know the muon momentum $p$ :

$$
r_{p}=\frac{p}{c B} \times 10^{3}
$$

For an ideal injection bunch of width of $120 \mathrm{~ns}$, we determine how long it takes to overlap itself. The revolution time for muons at the outside of the aperture with radius of $r_{\text {outerEdge }}=7157.0 \mathrm{~mm}$ is about

$$
t_{\text {outerEdge }}=150.0860541 \text { ns }
$$

and the revolution time for muons at the inside of the aperture with radius of $r_{\text {innerEdge }}=$ $7067.0 \mathrm{~mm}$ is about:

$$
t_{\text {innerEdge }}=148.1987068 \text { ns }
$$

Therefore, the revolution difference for muons between the inside of the aperture and the outside of the aperture for each turn is $\Delta=t_{\text {outEdge }}-t_{\text {innerEdge }}$. Hence, the time for overlap is

$$
t_{\text {overlap }}=\frac{\tau_{\text {revolution }}-t_{\text {pulseWidth }}}{\Delta} \times \tau_{\text {revolution }} \approx 2302 \mathrm{~ns}
$$

This means that after around 15 turns $(2.3 \mu s)$, the beam starts to overlap itself. For fast rotation, this seems to be "bad" because it will be hard to distinguish the overlapped bunches. However, in our analysis, if we consider a small segment of the injection pulse, the overlap time can be much longer. Then we can have a large range of fast rotation signals to use.

\section{Toy Monte Carlo Model:}

Based on the knowledge of the muon revolution and the detection of the decay positron signal, we consider a Toy Monte Carlo Model to test the $\chi^{2}$ minimization method. Both a Gaussian-shape injection pulse and a "W-shape" injection pulse are considered. Here, we

will focus on the latter. The injection pulse we use is shown in Fig. 5.9. The width of the "W"-shape is 120 ns.

We let the muon beam be injected into the storage ring and the beam will cycle around the ring under the magnetic field. Muons will decay into positrons and there exists a correlation between the muon spin and decay positron direction. With an energy threshold, decay 


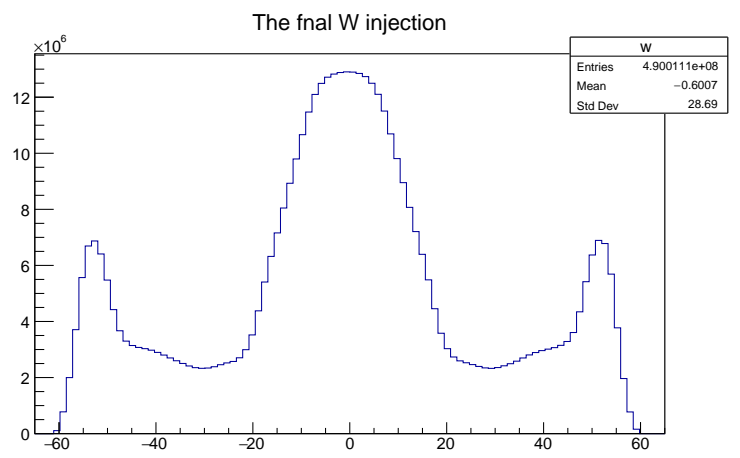

Figure 5.9: Injection pulse with a "W" shape used for simulation.

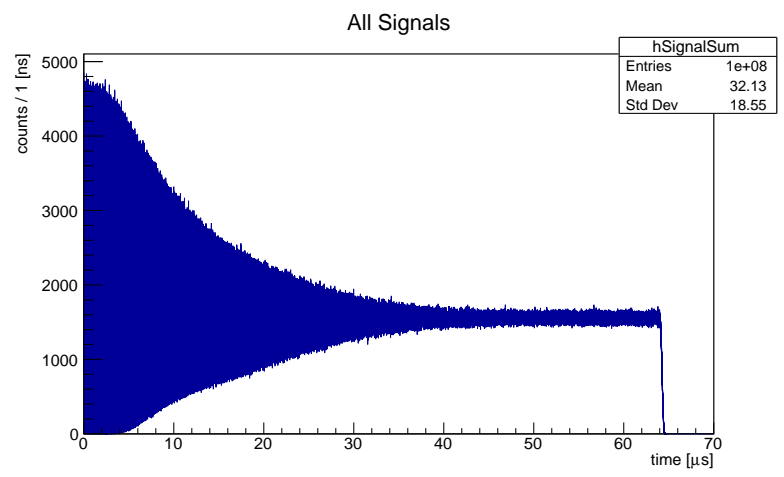

Figure 5.10: Decay positron signal seen by the calorimeters.

positrons in a range of angles will bend towards the center and be seen by the calorimeters around the ring. The decay positron histogram is shown in Fig. 5.10. If we zoom in, we will see the beam bunches are separated at early time and overlap at late time, as shown in Fig. 5.11. The bunch shapes at early time are very close to the injection pulse shape. This is very useful information. We can rebuild the injection pulse shape from the decay positron signal at early times if we are not able to obtain it earlier. Even though we have the injection pulse shape, the rebuilt bunch shape is also useful if we want to make a comparison with the injection pulse shape and if we start our fast rotation analysis at some late time, i.e., after the scraping study. The bunches will overlap with their neighbors and eventually overlap with themselves since they have a radial spectrum and muons at different orbits have different revolution periods.

In order to find the geometry factors $\beta_{i j}$, we need to know the injection pulse shape and the injection time $t_{0}$. For the former, we can either obtain it from the upstream beamline or rebuild it using the decay positron signal at very early time. For the latter, we try to fit the arrival time of bunches by number of turns with the following linear relation:

$$
t_{\text {peak }}=n T_{C}+t_{0}
$$

where $T_{C}$ is the bunch revolution time and the $t_{\text {peak }}$ is the peak time of each bunch and 

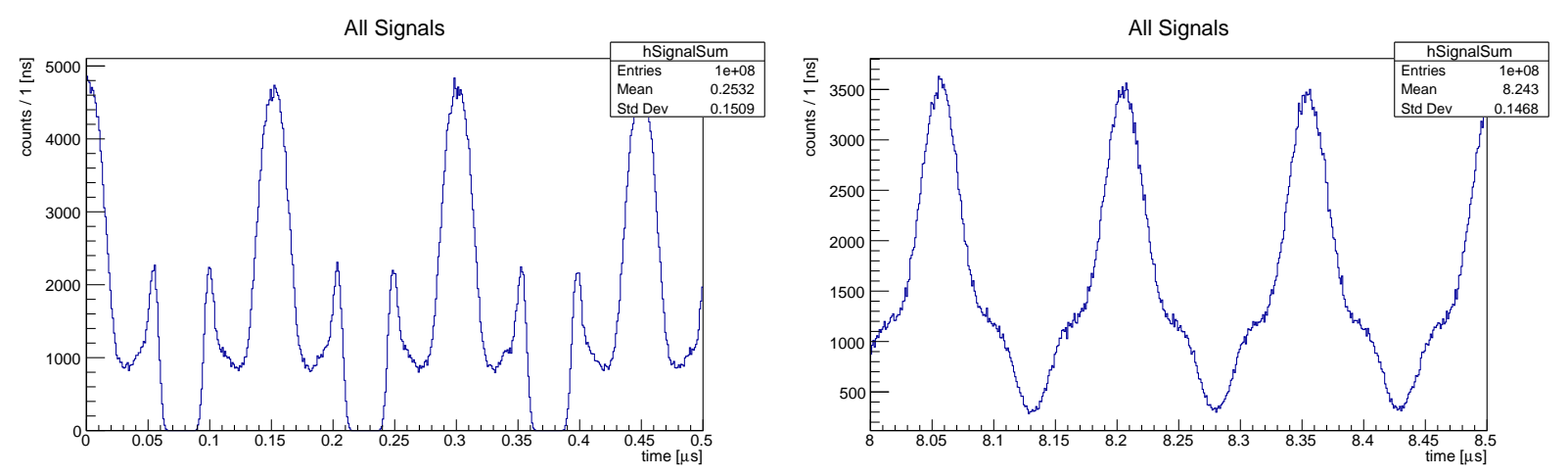

Figure 5.11: Decay positron signal seen by the calorimeters at different time: early time (left) and late time (right).

stands for the arrival time of the bunch. Here, $t_{\text {peak }}$ may not be the exact arrival time. It is just a reference time point for the bunch. Indeed, we could choose any point in the bunch as a reference.

The standard least squares method is used to calculate the sum of the squares of the vertical deviations of a set of $m$ data points:

$$
\chi^{2}=\sum_{j=1}^{m} \frac{\left[t_{j}-\left(n_{j} T_{C}+t_{0}\right)\right]^{2}}{\sigma_{j}^{2}}
$$

The condition of the minimum is that

$$
\frac{\partial \chi^{2}}{\partial T_{C}}=-2 \sum_{j=1}^{m} \frac{\left[t_{j}-\left(n_{j} T_{C}+t_{0}\right)\right] n_{j}}{\sigma_{j}^{2}}=0
$$

and

$$
\frac{\partial \chi^{2}}{\partial t_{0}}=-2 \sum_{j=1}^{m} \frac{\left[t_{j}-\left(n_{j} T_{C}+t_{0}\right)\right]}{\sigma_{j}^{2}}=0
$$

The above two equations lead to the following:

$$
\begin{array}{r}
m \sum_{j=1}^{m} \frac{t_{0}}{\sigma_{j}^{2}}+T_{C} \sum_{j=1}^{m} \frac{n_{j}}{\sigma_{j}^{2}}=\sum_{j=1}^{m} \frac{t_{j}}{\sigma_{j}^{2}} \\
t_{0} \sum_{j=1}^{m} \frac{n_{j}}{\sigma_{j}^{2}}+T_{C} \sum_{j=1}^{m} \frac{n_{j}^{2}}{\sigma_{j}^{2}}=\sum_{j=1}^{m} \frac{n_{j} t_{j}}{\sigma_{j}^{2}}
\end{array}
$$



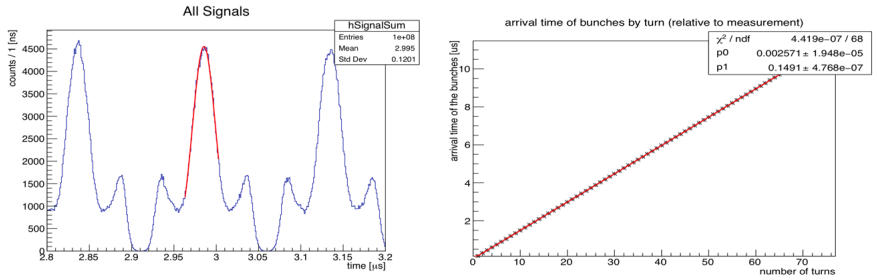

Figure 5.12: Toy Monte Carlo model: determination of $t_{0}$ and $T_{C}$.

By solving the above equations, we can obtain $t_{0}$ and $T_{C}$. The results are

$$
\begin{aligned}
& t_{0}= \frac{\sum_{j=1}^{m} \frac{t_{j}}{\sigma_{j}^{2}} \sum_{j=1}^{m} \frac{n_{j}^{2}}{\sigma_{j}^{2}}-\sum_{j=1}^{m} \frac{n_{j}}{\sigma_{j}^{2}} \sum_{j=1}^{m} \frac{n_{j} t_{j}}{\sigma_{j}^{2}}}{\sum_{j=1}^{m} \frac{1}{\sigma_{j}^{2}} \sum_{j=1}^{m} \frac{n_{j}^{2}}{\sigma_{j}^{2}}-\left(\sum_{j=1}^{m} \frac{n_{j}}{\sigma_{j}^{2}}\right)^{2}} \\
& T_{C}=\frac{\sum_{j=1}^{m} \frac{1}{\sigma_{j}^{2}} \sum_{j=1}^{m} \frac{n_{j} t_{j}}{\sigma_{j}^{2}}-\sum_{j=1}^{m} \frac{n_{j}}{\sigma_{j}^{2}} \sum_{j=1}^{m} \frac{t_{j}}{\sigma_{j}^{2}}}{\sum_{j=1}^{m} \frac{1}{\sigma_{j}^{2}} \sum_{j=1}^{m} \frac{n_{j}^{2}}{\sigma_{j}^{2}}-\left(\sum_{j=1}^{m} \frac{n_{j}}{\sigma_{j}^{2}}\right)^{2}}
\end{aligned}
$$

The errors of the parameters are given by

$$
\begin{gathered}
\delta t_{0}=\sum_{j=1}^{m} \frac{\partial t_{0}}{\partial t_{j}} \delta t_{j}=\sqrt{\frac{\sum_{j=1}^{m} n_{j}^{2}}{\sum_{j=1}^{m} \frac{1}{\sigma_{j}^{2}}\left[\sum_{j=1}^{m} \frac{1}{\sigma_{j}^{2}} \sum_{j=1}^{m} \frac{n_{j}^{2}}{\sigma_{j}^{2}}-\left(\sum_{j=1}^{m} \frac{n_{j}}{\sigma_{j}^{2}}\right)^{2}\right]}} \\
\delta T_{C}=\sum_{j=1}^{m} \frac{\partial T_{C}}{\partial t_{j}} \delta t_{j}=\sqrt{\frac{m}{\sum_{j=1}^{m} \frac{1}{\sigma_{j}^{2}}\left[\sum_{j=1}^{m} \frac{1}{\sigma_{j}^{2}} \sum_{j=1}^{m} \frac{n_{j}^{2}}{\sigma_{j}^{2}}-\left(\sum_{j=1}^{m} \frac{n_{j}}{\sigma_{j}^{2}}\right)^{2}\right]}}
\end{gathered}
$$

Note that the errors should be checked within the program which selects a bunch pulse. The $\sigma_{j}$ is the error on the peak center, which is defined by

$$
\sigma_{j}^{2}=\frac{\sum n_{i}\left(t_{i}-\bar{t}_{i}\right)}{\sum n_{i}}
$$

To calculate the peak time of each bunch $\left(t_{\text {peak }}\right)$, we can try several different methods, i.e., fitting with a sine function or using the ROOT module to find the peak location. However, because the bunch shape has a tail at each end, the more accurate method is to select the middle part of the bunch and fit that with a Gaussian for each bunch. The peak time $t_{\text {peak }}$ will be the mean value. Once we have the time information, we can convert the time to number of turns and fit the data with the linear relation in Eq. 5.22. The results are given in Fig. 5.12.

Once we have the injection time and the injection pulse information, we can calculate the geometry factors $\beta_{i j}$ and apply the $\chi^{2}$ minimization analysis to solve for the radial (momentum) bin contents. The result is given in Fig. 5.13.

To check the results, we can compare the expected counts by using the radial distribution 


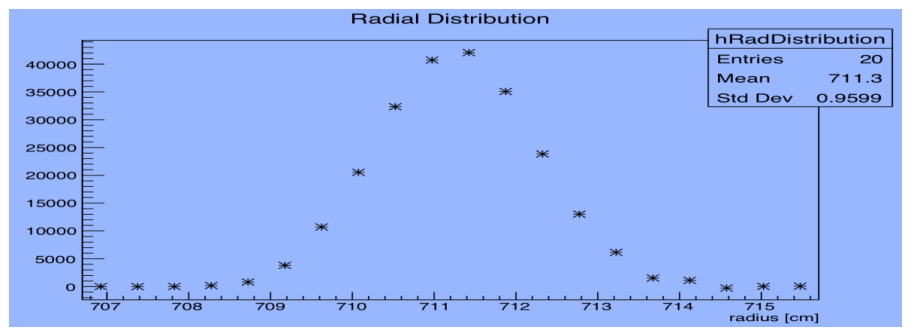

Figure 5.13: Toy Monte Carlo model: radial distribution.
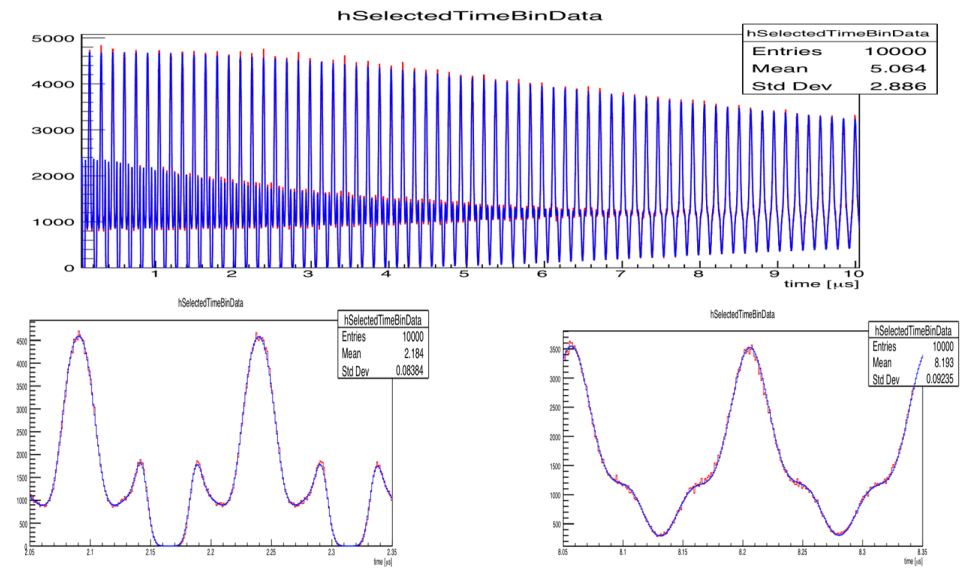

Figure 5.14: Toy Monte Carlo model: results check at different time scales. Red: decay positron counts seen by calorimeters; Blue: expected counts from fast rotation analysis.

shown in Fig. 5.13 with that seen by the calorimeters. The result is given in Fig. 5.14. As we can see from different time ranges, they agree with each other very well. This means that the fast rotation analysis can give a reliable radial momentum distribution.

\section{Simulation with gm2ringsim:}

In the experiment, we have a gm2ringsim package using GEANT4 for the simulation. The idea of the gm2ringsim is very similar to that of the toy Monte Carlo model. In fact, we can use the exponential decay of the muon in the toy Monte Carlo model. The gmaringsim can include the muon lifetime, $g-2$ frequency, muon loss and the CBO frequency, which can be backgrounds of the fast rotation analysis. For the fast rotation analysis, we will try to remove them by dividing from the decay positron histogram. The details of the procedure will be introduced in the following section. The results of using gm2ringsim are shown in Fig. 5.15, which agree with the toy Monte Carlo model.

\subsubsection{Commissioning Data}

Commissioning Run in Summer 2017: 


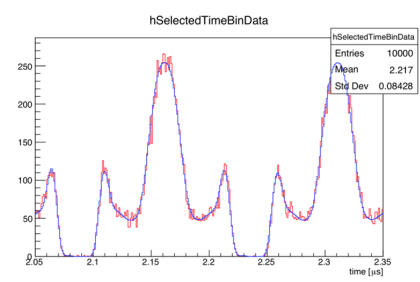

(a) Early time

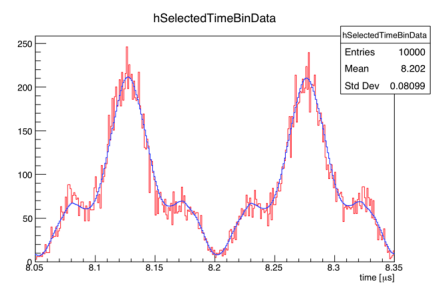

(b) Late time

Figure 5.15: Example: gm2ringsim simulation results. Red: decay positron counts seen by calorimeters; Blue: expected counts from fast rotation analysis.

The Fermilab Muon $g-2$ Experiment completed a commissioning (engineering) run in Summer 2017 (May-July). The main purpose was to test each subsystem, such as the Ring Magnet, Kicker, Quads, etc. During the commissioning run, the injected beam going into the muon storage ring is a mixture of protons, pions and muons (during this commissioning run, the beam was not sent to the Delivery Ring to remove the protons and pions). The ratio of protons to muons is about 60:1. The measured number of stored muons was about a factor of 40 lower than the TDR estimated value [97]. This loss factor is in a systematic manner and includes loss in pion production, beam transmission, beam injection and storage. Understanding the loss factor is very important for the physics runs.

How to Align Calorimeters:

For fast rotation analysis, we can work on the data on a single calorimeter. However, the statistics of the commissioning run in summer 2017 is very small. To get a relatively larger dataset, we need to align the 24 calorimeters. In other words, we need to add signals from those 24 calorimeters. In fact, a sum over all calorimeters can help us to reduce the effect from the CBO on the fast rotation analysis, as discussed in Section 4.2.1.

The 24 calorimeters are located symmetrically inside the storage ring. Ideally, the time shift from one calorimeter to the next one is about $6.2 \mathrm{~ns}\left(T_{C} / 24\right.$ with the magic $T_{C}=$ $0.149143 \mu \mathrm{s})$. To align calorimeters, we can just select a reference calorimeter, and then shift the time of the other calorimeters according to it. However, if the beam center is not at the center of the storage ring, this will introduce a time error, but the error should be very small because the storage aperture is only $90 \mathrm{~mm}$ wide and the worst case is about \pm 0.04 ns. Another possible way to align the calorimeters is using the injection time found for each calorimeter. If the data quality is reliable, this should give us the same result as the method of shifting the time using their locations.

The Revised Fast Rotation Signals:

For the decay positrons, we apply an energy cut of $E_{e^{+}}>1.8 \mathrm{GeV}$ to select those positrons in a range of angles in the muon rest frame. Because of the correlation between the muon spin and decay positron directions, the selected decay positron signals can be used to study the muon anomalous spin precession frequency. A general decay positron energy histogram is given in Fig. 5.16. After the energy cut, a typical decay positron time histogram contains 


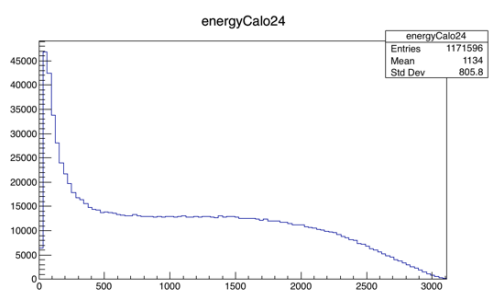

Figure 5.16: Example: decay positron energy histogram.

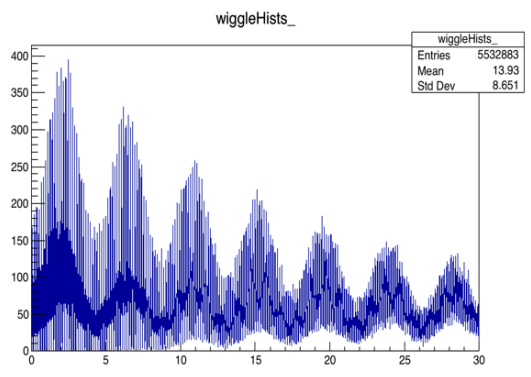

Figure 5.17: Example: decay positron time histogram.

the muon lifetime, the $g-2$ frequency, the $\mathrm{CBO}$, muon loss and the fast rotation, as shown in Fig. 5.17. Here, the fast oscillation is the cyclotron rotation and the slow oscillation is the $(g-2)$ precession. The slow decrease in rate comes from the decay of muons.

To get a constant rate on average and more reliable stored muon momentum distribution, we need to remove the effects of the muon lifetime as well as the $(g-2)$ precession from the fast rotation signals. If we can functionalize those backgrounds, we can remove them for our fast rotation analysis. Assuming that $S(t)$ is the signal of decay positron counts from detectors, we can fit this with an ideal signal function

$$
S_{F i t}(t)=N e^{-t / \tau}\left[1+A \cos \left(\omega_{a}+\phi\right)\right]
$$

The revised fast rotation signals after removing the muon lifetime as well as the $(g-2)$ precession is given by

$$
F(t)=\frac{S(t)}{S_{F i t}(t) / N}=\frac{S(t)}{e^{-t / \tau}\left[1+A \cos \left(\omega_{a}+\phi\right)\right]}
$$

Here, we keep $N$ in the revised fast rotation function to maintain the decay positron count information. Fig. 5.18 shows the fitted wiggle plot according to Eq. (5.33). The muon loss background can be removed in the same way once we have the muon loss function.

Results of Commissioning run in Summer 2017:

First, we find the difference between the proton beam and muon beam. According to Eq. (5.17), if the beam travels at the mean radius of the muon storage ring, its momentum is 
T-method wiggle plot chi2 minimization fit

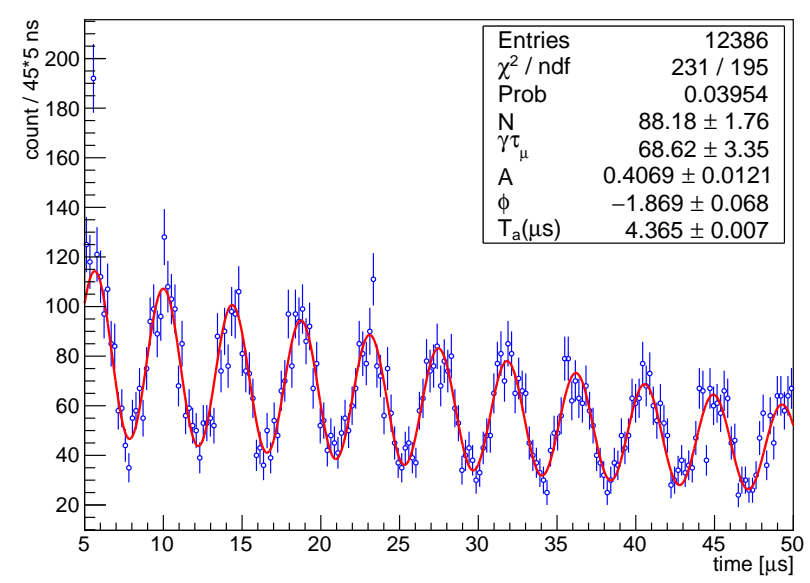

Figure 5.18: Example: Fit wiggle plot (ideal signal function) to run 1462 data.

given by

$$
p_{r}=r c B * 10^{-3}=7112 \times 299.792458 \times 1.451269 \times 10^{-3}=3094.29 \mathrm{MeV} / \mathrm{c}
$$

In Table 5.1, we list some basic information about the proton and muon at the magic radius.

\begin{tabular}{|l|l|l|l|l|l|l|}
\hline Beam & Mass $\left[\mathrm{MeV} / \mathrm{c}^{2}\right]$ & momentum $[\mathrm{MeV} / \mathrm{c}]$ & Revolution Time $[\mathrm{ns}]$ & frequency $[\mathrm{MHz}]$ & Beta factor & Gamma factor \\
\hline Proton & 938.2720813 & 3094.29 & 155.758 & 6.4202 & 0.956972 & 3.44614 \\
\hline Muon & 105.6583715 & 3094.29 & 149.1433667 & 6.705 & 0.9994168695 & 29.28636415 \\
\hline
\end{tabular}

Table 5.1: Summary of the muon beam and proton beam at mean radius.

Sometimes, it is hard to find the injection pulse shape. To understand the bunch structure better, it is necessary to select some individual beam bunch pulses, from which, we can construct the bunch pulse shape as well as study its evolution. We can use the early bunch pulse to rebuild the injection pulse. The more events we have, the more accuracy we can achieve. Several bunch pulses may be added to find the injection pulse shape. A test is given in Fig. 5.19.

To find the injection time and the bunch revolution time, we use the method discussed in Section 5.3.5. We fit the arrival time of the beam bunches by number of turns with the linear relation: $t_{p e a k}=n T_{C}+t_{0}$, where $n$ is the number of turns, $T_{C}$ is the average revolution time and $t_{0}$ the bunch injection time. We could also start with the first full bunch pulse and limit $\frac{T_{C}}{2} \leq t_{0}<T_{C}$. Although this is not really required, it can give us a reference to the time of the injection pulse. We use the peak of the bunch pulse to represent the arrival time. In this case, we have to be careful to avoid the tail peaks of the bunch. We can try to fit a sine function to some bunch pulses to get the revolution time:

$$
F(t)=A \sin \left[\frac{2 \pi}{T_{C}}\left(t-t_{0}+\frac{T_{C}}{4}\right)\right]
$$




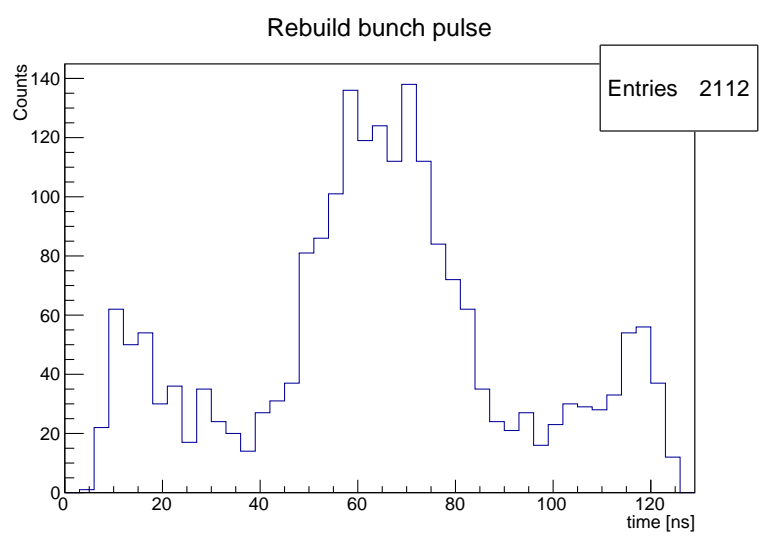

Figure 5.19: Rebuilt injection pulse shape using the first few bunch pulses (from simulation).

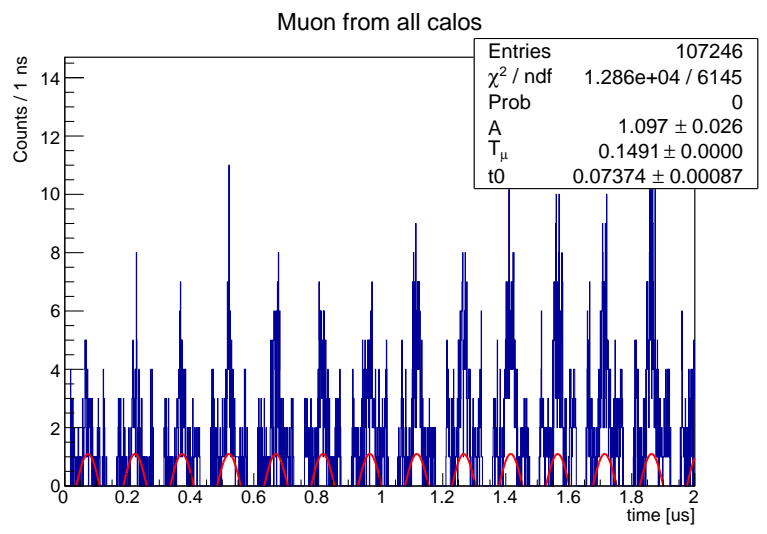

Figure 5.20: Example: fitting a sine function to extract the $T_{C}$ and $t_{0}$.

An example of this is shown in Fig. 5.20. We can also try the "TSpectrum" module in ROOT, which can find the peaks of the spectrum and return their positions. An example of using "TSpectrum" is shown in Fig. 5.21. The fitted revolution time is 149.6 ns. Here, we have skipped the very beginning time that contains lots of protons or other backgrounds for selecting muon events.

For the proton events, we can apply the same analysis to obtain the revolution time for the proton beam. An example is shown in Fig. 5.22 using the data of commissioning run 1462. The revolution time obtained for the proton bunch is $155.8 \mathrm{~ns}$, which is a little larger than the proton revolution time at the mean radius Table 5.1. We can perform a Fast Fourier Transform (FFT), which can give us the revolution frequency information, as shown in Fig. 5.23 .

The fast rotation analysis requires a large dataset of decay positron events. In addition to the sum over all calorimeters, we also combined many runs with the same setting for the commissioning run in Summer 2017. However, the statistics of the data we have is still very small. We can only perform a rough fast rotation analysis. Because the injection pulse is unstable or unknown, we rebuilt the pulse shape as discussed above. Then we fitted the 


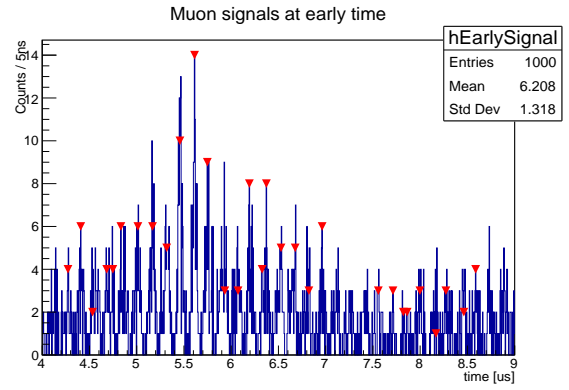

(a) Muon signals at early time; Red triangles represent bunch arrival time (peak).

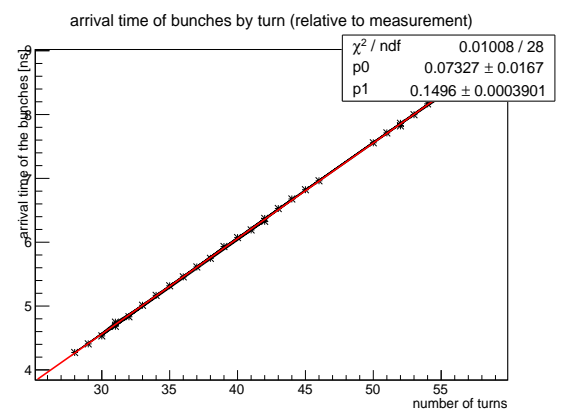

(b) Fitting with Eq. (5.22) of muon signals at early time.

Figure 5.21: Commissioning Run 1462: using "TSpectrum" to determine $t_{0}$ and $T_{C}$ for muon signals.

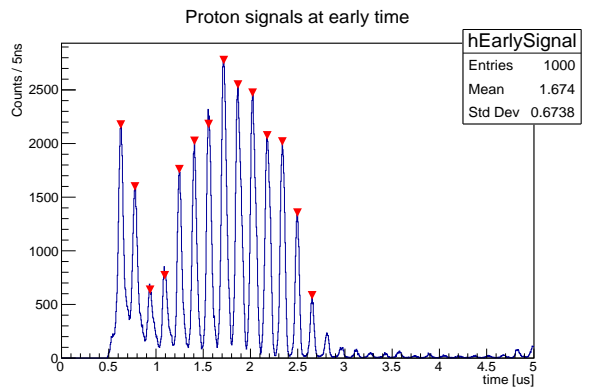

(a) Proton signals at early time; Red triangles represent bunch arrival time (peak).

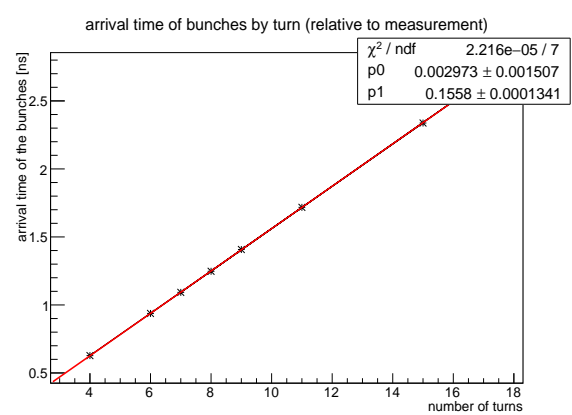

(b) Fitting with Eq. (5.22) of proton signals at early time.

Figure 5.22: Commissioning Run 1462: using "TSpectrum" to determine $t_{0}$ and $T_{C}$ for proton signals.

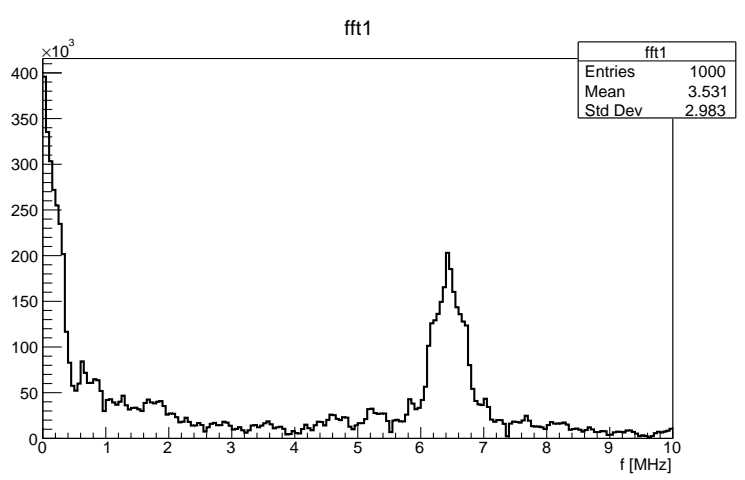

Figure 5.23: Example: Fourier Transform of proton signals in run 1462. 


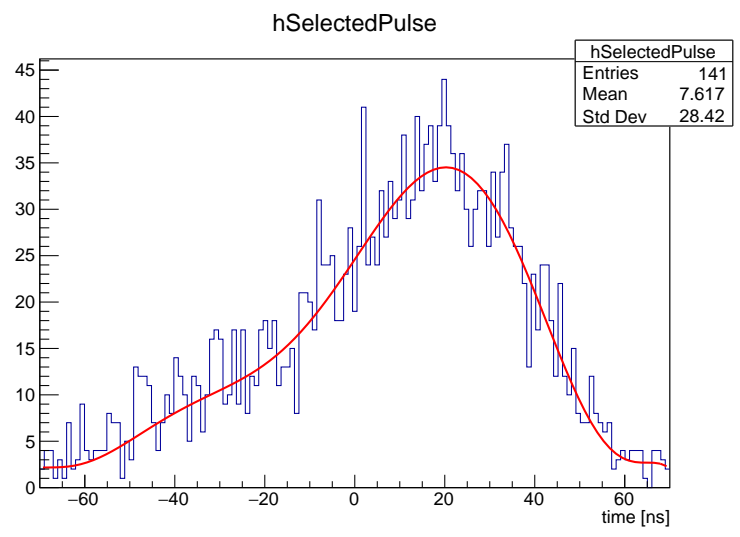

Figure 5.24: Example: new pulse shape rebuilt for fast rotation analysis (commissioning data in summer 2017).

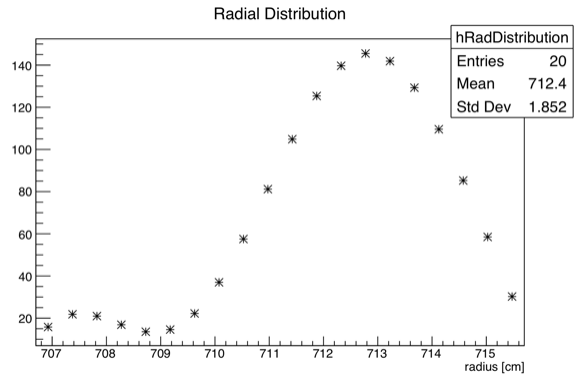

Figure 5.25: Example: radial distribution (commissioning data in summer 2017).

rebuilt pulse shape with a "polyN" to create the new "injection" pulse, as shown in Fig. 5.24. The injection time $t_{0}$ was found by fitting the arrival time of beam bunches by number of turns, where the arrival time was determined by the peak time $t_{\text {peak }}$ using a Gaussian fit. The radial distribution for the muons is shown in Fig. 5.25. The mean value of the radius is about $18 \mathrm{~mm}$ larger than the magic radius. This was also confirmed by the Tracker data. This means the beam was away from the center, and indicates why we could not store more muons. A summary of the fast rotation analysis of the commissioning run data of Summer 2017 can be found in Ref. [98].

\section{Commissioning for Physics Run FY18:}

The commissioning for physics run FY18 started in November 2017. The main purpose of this commissioning is to achieve a stable decay positron event rate for the physics run FY18. This includes solving for the muon loss factor that was found in the commissioning run in Summer 2017 and maintaining a working system, including the operation of Quads and Kickers. Up to March 15, 2018, we still worked on commissioning (The Physics Run FY18 started on March 22). However, the event rate already meets about $40 \%$ of the TDR requirement. In other words, we can achieve about twice the BNL $g-2$ statistics at our current event rate for the physics run FY18. The beam is sent to the Delivery Ring before 

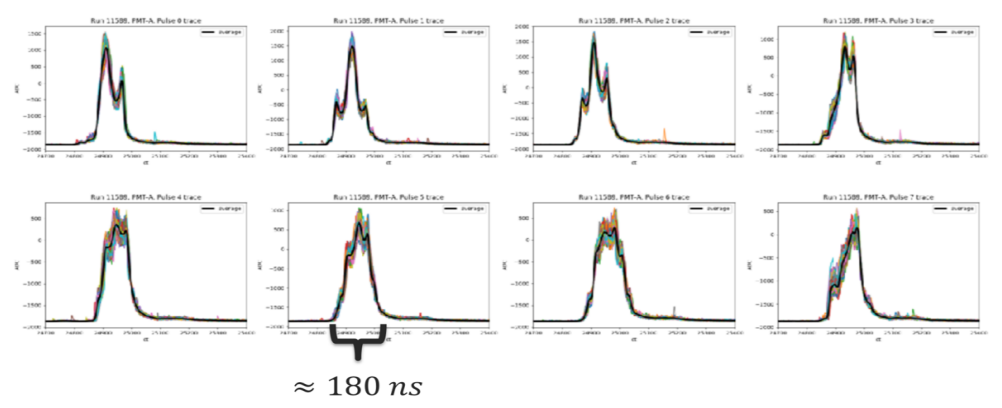

Figure 5.26: Example: T0 pulses of Commissioning for physics run FY18 [99].

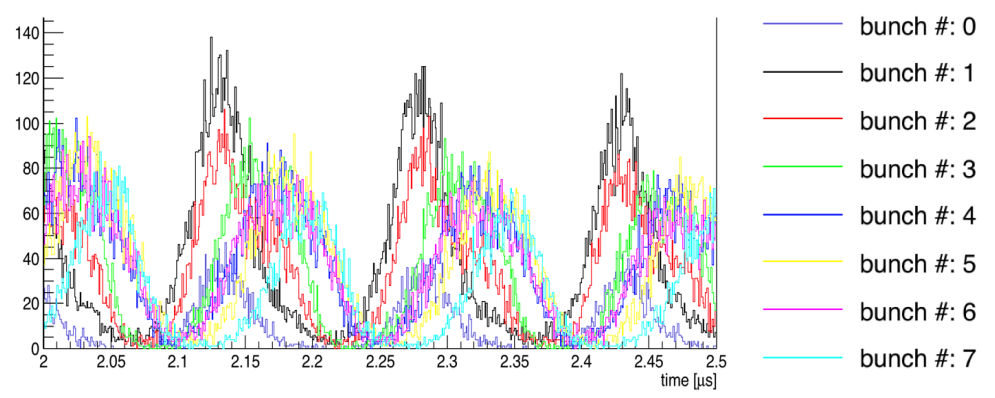

Figure 5.27: Example: decay positron signal from each bunch at early time.

being injected into the storage ring. Therefore, there are no protons in the injection beam. Almost all the pions are also removed in the Delivery Ring. The injection beam is mainly muons but may contains some positrons. Positrons will be lost after several turns. Hence, the beam quality is much better than that in summer 2017 .

For the fast rotation analysis, we follow the procedure discussed above. We also notice that our 8 bunch pulses are very different from each other and they are not the expected "W-shape", as shown in Fig. 5.26. We want to determine if the muon radial distribution obtained from FRA depends at all on the bunch number. In reality, if different bunches have different T0 shapes, the stored muon beam profiles both in the time dimension and the radial dimension may change from bunch to bunch, mainly due to the behavior of our Kickers. In other words, the detectors will see different decay positron signals from each bunch, as shown in Fig. 5.27.

As an example, we look at the runs of SuperBowlFY18, which are the Runs 11169-11177 and have the nominal beam setup, i.e., scraping on, nominal Kicker strength, etc. The decay positron signal from one bunch is shown in Fig. 5.28. To remove the muon lifetime and $g-2$ frequency effects on the fast rotation signal, we fit an ideal wiggle function to the histogram in Fig. 5.28. The result is given in Fig. 5.29.

After removing the muon lifetime and the $g-2$ frequency, we can rebuild the "injection" pulse time shape, as shown in Fig. 5.30. Here, the pulse shape we rebuild is the pulse seen by the detectors at some time, which may be different from the pulse shape at the beam injection time. We apply the same analysis on $t_{0}$ and $T_{C}$. The radial distribution for bunch \#0 is 


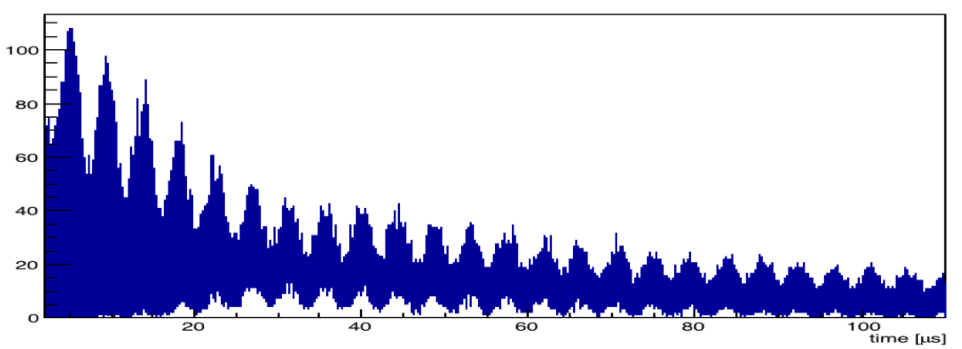

Figure 5.28: SuperBowlFY18: decay positron signal from bunch \#0.

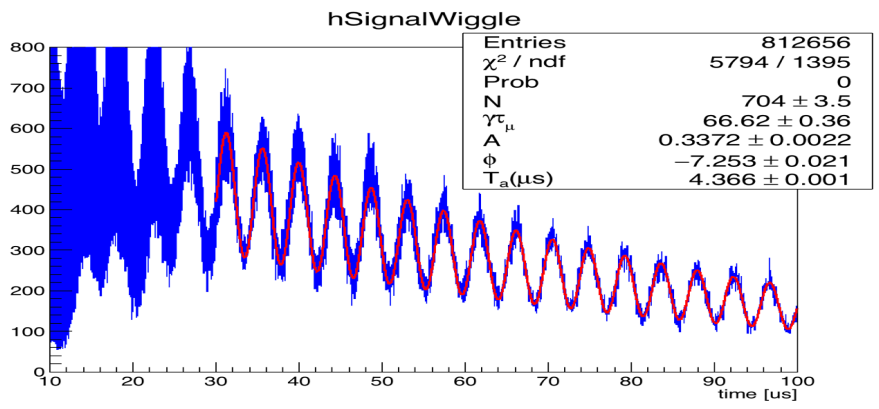

Figure 5.29: SuperBowlFY18: fitting the wiggle function to the decay positron signal from bunch \#0.

shown in Fig. 5.31. The mean radius is still about $0.8 \mathrm{~mm}$ higher than the magic radius. The tails at each side are due to small statistics and inaccurate injection pulse shape, not to a physical issue. When the statistics of events are too small, the parameters that describe the relations between the neighbor radial bins in the fast rotation model cannot represent the radial distribution properly and the rebuilt injection pulse may miss some features of the real pulse.

The commissioning for physics run FY18 has 8 bunches (16 for physics run). We follow the above procedure to perform the fast rotation analysis for each bunch by looking at the decay positron data around $2 \mu$ s after the beam injection into the storage ring. The preliminary results are shown in Fig. 5.32, from which we can learn the radial distributions are slightly different for different bunches. This should be studied and verified in the data analysis of physics run FY18.

Another interesting fast rotation analysis is to look at the decay positron signal with a different starting time. The motivation here is that the Kickers are only turned on at the first turn and the scraping is only turned on for the first few microseconds. Our beam is much more stable at later times. In fact, most of the muon losses occur at early times. This will affect the fast rotation analysis. As an example, we look at the decay positron signal from bunch \#3 in the SuperBowlFY18 dataset. The results are given in Fig. 5.33, from which we see that the distribution depends on the starting time points. Though more careful analysis is needed here, we are not surprised and we should always keep in mind that the beam dynamics is much more complex at early time compared with that at late time. The 


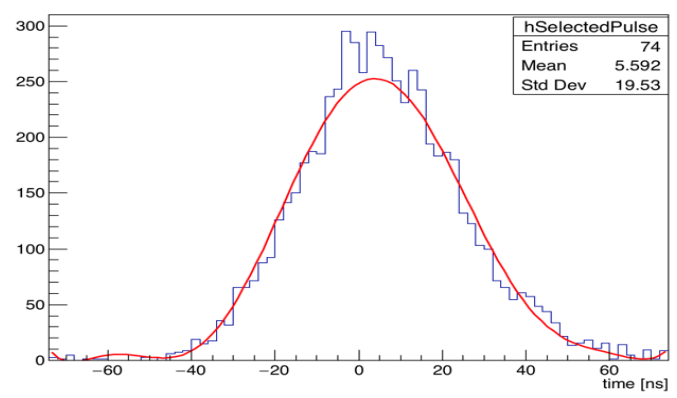

Figure 5.30: SuperBowlFY18: Rebuild pulse shape (not real "injection" shape) for bunch $\# 0$.

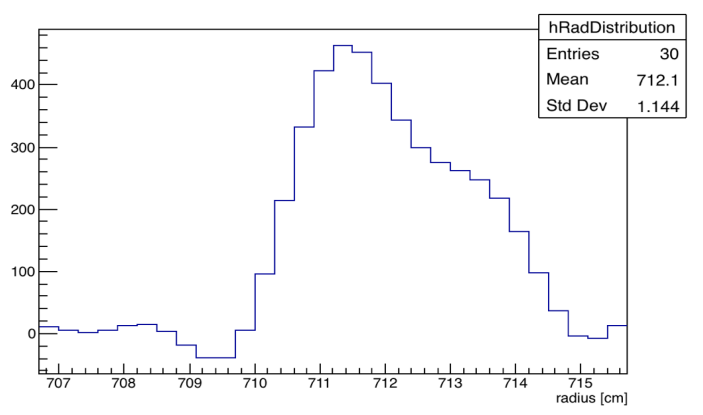

Figure 5.31: SuperBowlFY18: muon radius distribution of bunch \#0.

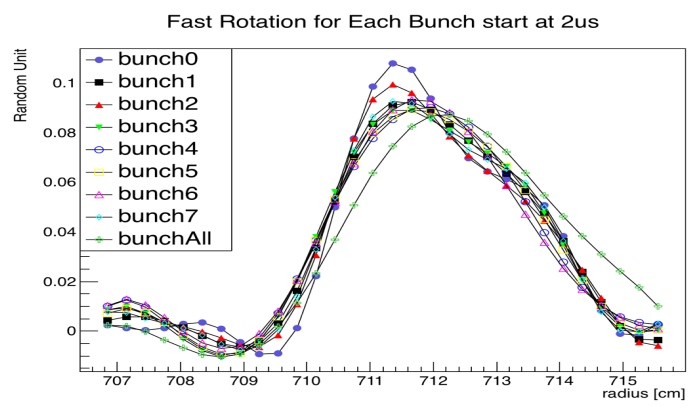

Figure 5.32: SuperBowlFY18: muon radius distribution of each bunch. 

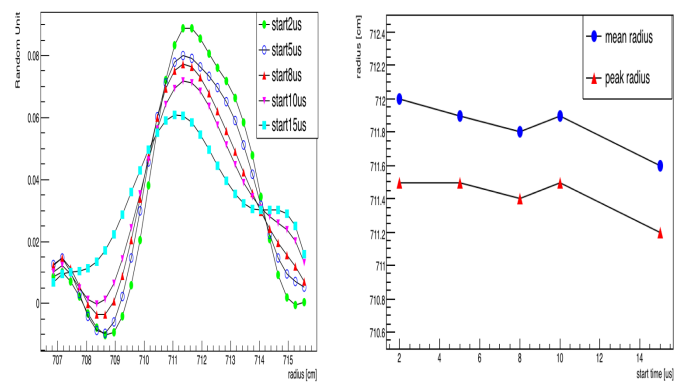

Figure 5.33: SuperBowlFY18: radius distribution for FRA starting at different time points of bunch \#3.

mean radius is higher than the magic radius. Muons have a large probability to be lost if they sit on a higher orbit. Here, the muon losses, as well as CBO, should be considered in order to do a further study of fast rotation analysis, i.e., whether the momentum distribution changes over time. More details of fast rotation analysis on the commissioning for physics run FY18 can be found in Ref. [100].

\subsection{Electric Field Correction}

The electric field correction to the measured $a_{\mu}$ comes from the third term in Eq. 2.6. To evaluate the effect of the electric field due to muons not exactly at the magic radius $\left(\gamma_{\text {magic }} \simeq 29.3\right)$, we neglect the second term of $\vec{\beta} \cdot \vec{B}$ at this moment. Therefore, we have

$$
\vec{\omega}_{a}=-\frac{q}{m}\left[a_{\mu} \vec{B}-\left(a_{\mu}-\frac{1}{\gamma^{2}-1}\right)\left(\frac{\beta \times \vec{E}}{c}\right)\right]
$$

If the muon momentum is different from the magic momentum $\left(\gamma_{\text {magic }} \neq 29.3\right)$, the anomalous spin precession frequency is given by

$$
\omega_{a}^{\prime}=\omega_{a}\left[1-\beta \frac{E_{r}}{c B_{y}}\left(1-\frac{1}{a_{\mu} \beta^{2} \gamma^{2}}\right)\right]
$$

where $\omega_{a}=-a_{\mu} \frac{q B}{m}$. Now recalling $p=\beta \gamma m=\left(p_{\text {magic }}+\Delta p\right)$, we can obtain (after some mathematical steps)

$$
\frac{\omega_{a}^{\prime}-\omega_{a}}{\omega_{a}}=\frac{\Delta \omega_{a}}{\omega_{a}}=-2 \frac{\beta E_{r}}{c B_{y}}\left(\frac{\Delta p}{p_{\text {magic }}}\right)
$$

Now, we consider the relation between the momentum and the radius:

$$
\frac{\Delta p}{p_{\text {magic }}}=(1-n) \frac{\Delta R}{R_{0}}=(1-n) \frac{x_{e}}{R_{0}}
$$


where $x_{e}$ is the muon's equilibrium radius relative to the central (magic) orbit. If we write the electric quadrupole field as

$$
E=\kappa x=\frac{n \beta c y}{R_{0}} x
$$

we then obtain

$$
\frac{\Delta \omega_{a}}{\omega_{a}}=-2 n(1-n) \beta^{2} \frac{x x_{e}}{R_{0}^{2}}
$$

The minus sign "-" here indicates that the effect of the muons not at the magic momentum is to lower the observed anomalous spin frequency. For a quadrupole focusing field plus a uniform magnetic field, the time average of $x$ is just $x_{e}$ [12]. Therefore, the electric field correction is given by

$$
C_{E} \equiv \frac{\Delta \omega_{a}}{\omega_{a}}=-2 n(1-n) \beta^{2} \frac{<x_{e}^{2}>}{R_{0}^{2}}
$$

Here, $\left\langle x_{e}^{2}>\right.$ can be determined from the fast rotation analysis. However, if we have a large $x_{e}$, the tails of the $x_{e}$ distribution will dominate and the uncertainty in $\bar{x}_{e}^{2}$ may become an issue. One may need to take that into account. Also, the E-field correction should be treated carefully if the quad plates are misaligned or uncertain in position, which can lead to a direct error in the E-field correction [101]. Using Eq. (5.43), one can easily calculate the effect of the electric field to muons not exactly at the magic momentum $\left(\gamma_{\text {magic }} \simeq 29.3\right)$. The electric field correction here is related to the field index and the mean horizontal position of the muon beam. 


\section{CHAPTER 6}

\section{CONCLUSION}

In this dissertation, the theory and experimental measurement of the muon $g-2$ are introduced. The principle and techniques of the Fermilab Muon $g-2$ Experiment are discussed and studies of the beam dynamics and the electric field related work are described. In this chapter we present a short summary of this work, its impacts on the E989 measurement and the outlook for Muon $g-2$.

\subsection{Electrostatic Quadrupole System}

In Chapter 3, we discussed the quad plate alignment and the quad extension and readout system. The electrostatic quadrupole system (ESQ) is very important for the experiment. It provides electric fields for vertical beam focusing inside the storage ring. The alignment of quad plates reduces the effects of higher order multipoles on the beam dynamics. Stable operation of ESQ is essential to maintain the experiment's schedule. If the quad plates are not well aligned, the electric field correction to muon $g-2$ will become detrimental to the overall experimental goal of $<70$ ppb systematic uncertainty.

We investigated the quad plate alignment by using a Capacitec tool. To meet the alignment requirement, micrometer tools were also designed. We developed the procedure of installation and alignment of the quad plates by using the micrometer tools. The final quad plate alignments were checked with a laser alignment system which shows the 3D deviation of the plates. The final quad alignment results are given in Appendix A, from which we can tell most of our quad plate alignments are better the required specifications and can directly reduce the uncertainty of the E-field correction. Moreover, the effects of higher order multipoles are reduced, which will limit muon losses as well as reduce the systematic errors of muon loss and coherent betatron oscillation (CBO). Though Q1 outer Mylar plates may have a large deviation due to the waviness effect, the real situation should be better due to the fact that the very thin Mylar plates will allow more muons to survive after the Inflector compared with the previous $0.5 \mathrm{~mm}$ aluminium plates. The alignment of the ESQ plates is critical to the beam dynamics, especially the muon losses, CBO, E-field and pitch corrections. In addition, we successfully installed the quad extension and readout system, including potting the resistors and the design of the spark detection circuit. The integrated ESQ is tested with a higher voltage than that at BNL, which is extremely important for stable operation of the storage ring.

Currently, the whole electrostatic quadrupole system has been installed successfully. The system can be operated stably and works well for the experiment's commissioning. With a good vacuum environment, it is stable and reliable enough for physics runs. The entire ESQ 
system plays a significant role in storing the muon beam and is very important to achieve the E989 measurement goal by impacting both the statistical (in a confined run period) and systematic errors.

\subsection{Beam Dynamics in Muon Storage Ring}

In Chapter 4, we started with the equation of motion and studied the muon beam dynamics in the muon storage ring. Beam dynamics is absolutely vital to the experiment. It is essential to understand the beam dynamics uncertainties and how to reduce them in order to achieve a high precision measurement of $g-2$.

Several beam related systematic errors were discussed, including the CBO, lost muons, E-field and pitch corrections. We have simulated a 3D electric fields using OPERA-3D software. It is used for many physics studies, such as beam resonances, scraping, etc., which will improve our simulation and analysis with its beautiful 3D features. Also, we discussed the principle of beam resonances. Based on the knowledge of the beam resonances, we have investigated the muon $g-2$ beam resonances and studied those resonances around the lower $n$ value by performing quad scans with and without scraping. Beam resonances are understood very well from both simulation and the commissioning and will allow us to reduce the muon losses and the related systematic errors. Once we are able to start the high $n$ value run, we can have a better beam focus and a smaller betatron oscillation. The relevant systematic errors will be refined.

The 3D electric field map is a major improvement for the Fermilab Muon $g-2$ Experiment. It provides the fringe field at the ends of the quad plates, which is not possible with a 2D field map. With the detailed geometry of the plates, cage and vacuum chamber, the 3D field map is much more accurate for the beam dynamics simulation and analysis.

\subsection{Fast Rotation Analysis}

In Chapter 5, the physics of the fast rotation analysis was described. The fast rotation analysis is essential to understand the beam dynamics and determine the electric field correction to $a_{\mu}$. We have focused on the $\chi^{2}$ minimization method and developed the corresponding fast rotation analysis.

We discussed the methods and assumptions of the fast rotation analysis and presented a detailed calculation of the geometry factors used for the analysis. A toy Monte Carlo model is built to test the $\chi^{2}$ minimization method. The more complex simulation is achieved by using the gm2ringsim simulation package. The results indicate that the fast rotation analysis can give us a reliable muon momentum distribution. We have applied the preliminary study of the fast rotation analysis on the commissioning run data. As we have learned, the results not only provide us the key input for the electric field correction to muon g-2, but help us understand elements of the beam dynamics better, such as why we are losing muons and what the beam position is. The systematic studies for the fast rotation analysis on individual beam bunches and different starting time points are presented, which are very useful for understanding our beam dynamics and the behavior of our systems such as Inflector, Kicker, and Quads. For example, we have learned that the beam has a higher mean radius than the 
magic radius, which could be rectified if we use a greater Kicker strength. The knowledge we have learned from the commissioning is particularly useful for the experiment. The fast rotation study will be critical to the experiment's high precision measurement goal.

The $\chi^{2}$ minimization method tool is ready. It has been one of the key inputs for understanding the pre-production data and continues to help us shape critical decisions for the future. Once we have the physics data, it will be able to provide us with the momentum distribution and allow us to estimate the electric field systematic error. The result of it can be compared and cross-checked with that from the Fourier transform method, as we have learned in the BNL $g-2$ experiment.

\subsection{Outlook}

Muon $g-2$ plays a unique role in placing stringent constraints on new physics. The result of the BNL $g-2$ experiment has already provided a very significant hint for this. Parallel to efforts on the theoretical calculation of muon $g-2$, the Fermilab Muon $g-2$ Experiment will measure the anomalous magnetic moment of the muon to an unprecedented precision level of $0.14 \mathrm{ppm}$. There will be 21 times more statistics compared to the BNL $g-2$ experiment and a four-fold improvement on the systematic errors. The Fermilab Muon $g-2$ Experiment is ready for the first period of physics run (FY18) to collect a dataset equivalent to BNL $g-2$. In the following few years, much work will be required to reach the experiment's ultimate goal, but it will also be a very exciting time for the experiment

and for the whole particle physics community as the measurement of muon $g-2$ helps us to understand nature better by significantly improving constraints on new physics. 


\section{BIBLIOGRAPHY}




\section{BIBLIOGRAPHY}

[1] G. Aad et al. (ATLAS Collaboration). "Observation of a new particle in the search for the Standard Model Higgs boson with the ATLAS detector at the LHC". In: Phys. Lett. B716 (2012), pp. 1-29. eprint: arXiv: 1207.7214 [hep-ex].

[2] S. Chatrchyan et al. (CMS Collaboration). "Observation of a new boson at a mass of $125 \mathrm{GeV}$ with the CMS experiment at the LHC". In: Phys. Lett. B B716 (2012), pp. 30-61. eprint: arXiv:1207.7235 [hep-ex].

[3] P. A. M. Dirac. "The quantum theory of the electron". In: Proc. Roy. Soc. Lond. A117 (1928), pp. 610-624. DOI: 10.1098/rspa.1928.0023.

[4] J. E. Nafe, E. B. Nelson, and I. I. Rabi. "The hyperfine structure of atomic hydrogen and deuterium". In: Phys. Rev. 71 (1947), p. 914.

[5] D. E. Nagle, R. S. Julian, and J. R. Zacharias. "The hyperfine structure of atomic hydrogen and deuterium". In: Phys. Rev. 72 (1947), p. 971.

[6] P. Kusch and H. M. Foley. "Precision measurement of the ratio of the atomic ' $g$ Values' in the ${ }^{2} P_{3 / 2}$ and ${ }^{2} P_{1 / 2}$ states of gallium". In: Phys. Rev. 72 (1947), p. 1256.

[7] J. Schwinger. "On quantum-electrodynamics and the magnetic moment of the electron". In: Phys. Rev. 73 (1948), p. 416.

[8] J. Schwinger. "The electromagnetic properties of the electron - radiative corrections to scattering". In: Phys. Rev. 76 (1949), p. 790.

[9] T. Aoyama et al. "Tenth-order QED contribution to the electron g-2 and an improved value of the fine structure constant". In: Phys. Rev. Lett. 109 (2012), p. 111808.

[10] R. Bouchendira et al. "New determination of the fine structure constant and test of the quandtume electrodynamics". In: Phys. Rev. Lett. 106 (2011), p. 080801.

[11] Fred Jegerlehner and Andreas Nyffeler. "The muon g-2". In: Phys. Rept. 477 (2009), pp. 1-110. eprint: arXiv:0902.3360 [hep-ph].

[12] J. Grange et al. (Muon g-2 Collaboration). Muon (g-2) Technical Design Report. eprint: arXiv: 1501.06858 [physics . ins-det] .

[13] C. Gnendiger, D. Stöckinger, and H. Stöckinger-Kim. "The electroweak contributions to $(g-2)_{\mu}$ after the Higgs-boson mass measurement". In: Phys. Rev. D88 (2013), p. 053005. eprint: arXiv: 1306.5546 [hep-ph].

[14] A. Czarnecki, W. J. Marciano, and A. Vainshtein. "Refinements in electroweak contributions to the muon anomalous magnetic moment". In: Phys. Rev. D67 (2003), p. 073006.

[15] A. Vainshtein. "Perturbative and nonperturbative renormalization of anomalous quark triangles". In: Phys. Lett. B569 (2003), pp. 187-193. 
[16] M. Gourdin and E. De Rafael. "Hadronic contributions to the muon $g$-factor". In: Nucl. Phys. B10 (1969), pp. 667-674.

[17] B. E. Lautrup and E. De Rafael. "Calculation of the sixth-order contribution from the fourth-order vacuum polarization to the difference of the anomalous magnetic moments of muon and electron". In: Phys. Rev. 174 (1968), p. 1835.

[18] M. Ablikim et al. (BESIII Collaboration). "Measurement of the $e^{+} e^{-} \rightarrow \pi^{+} \pi^{-}$cross section between 600 and $900 \mathrm{MeV}$ using initial state radiation". In: Phys. Lett. B753 (2016), pp. 629-638.

[19] K. Hagiwara et al. " $(g-2)_{\mu}$ and $\alpha\left(M_{Z}^{2}\right)$ re-evaluated using new precise data". In: $J$. Phys. G38 (2011), p. 085003.

[20] M. Knecht and A. Nyffeler. "Hadronic light-by-light corrections to the muon $g-2$ : the pion-pole contribution". In: Phys. Rev. D65 (2002), p. 073034. eprint: arXiv : 0111058 [hep-ph].

[21] M. Davier et al. "Reevaluation of the hadronic contributions to the muon $g-2$ and to $\alpha\left(M_{Z}^{2}\right)$ ". In: Eur. Phys. J. C71 (2011), p. 1515.

[22] J. Prades, E. de Rafael, and A. Vainshtein. "Hadronic light-by-light scattering contribution to the muon anomalous magnetic moment". In: (). eprint: arXiv : 0901. 0306 [hep-ph].

[23] A. Czarnecki and W. J. Marciano. "Muon anomalous magnetic moment: A harbinger for 'new physics'". In: Phys. Rev. D64 (2001), p. 013014.

[24] S. P. Martin. "A Supersymmetry primer". In: (1997). DOI: 10.1142/9789812839657_ 0001,10.1142/9789814307505_0001. eprint: arXiv:9709356 [hep-ph].

[25] K. Harigaya, T. T. Yanagida, and N. Yokozaki. "Muon g-2 in focus point SUSY". In: Phys. Rev. D92 (2015), p. 035011. eprint: arXiv: 1505.01987[hep-ph].

[26] U. Chattopadhyay and P. Nath. "Probing supergravity grand unification in the Brookhaven g-2 experiment". In: Phys. Rev. D53 (1995), pp. 1648-1657. eprint: arXiv: 9507386 [hep$\mathrm{ph}]$.

[27] F. Jegerlehner. "The anomalous magnetic moment of the muon". In: Springer Tracts Mod. Phy. 274 (2017), pp. 1-693. DOI: 10.1007/978-3-319-63577-4.

[28] M. Pospelov. "Secluded U(1) below the weak scale". In: Phys. Rev. D80 (2009), p. 095002. eprint: arXiv:0811.1030 [hep-ph].

[29] M. Endo, K. Hamaguchi, and G. Mishima. "Constraints on hidden photon models from electron g-2 and hydrogen spectroscopy". In: Phys. Rev. D86 (2012), p. 095029. eprint: arXiv: 1209.2558[hep-ph].

[30] A. Datta, J. Liao, and D. Marfatia. "A light $Z^{\prime}$ for the $R_{K}$ puzzle and nonstandard neutrino interactions". In: Phys. Lett. B768 (2017), pp. 265-269. eprint: arXiv: 1702. 01099 [hep-ph].

[31] J. Heeck. "Lepton flavor violation with light vector bosons". In: Phys. Lett. B758 (2016), pp. 101-105. eprint: arXiv: 1602.03810 [hep-ph]. 
[32] R. L. Garwin, L. M. Lederman, and M. Weinrich. "Observations of the failure of conservation of parity and charge conjugation in meson decays: the magnetic moment of the free muon". In: Phys. Rev. 105 (1957), p. 1415.

[33] R. L. Garwin et al. "Accurate determination of the $\mu^{+}$magnetic moment". In: Phys. Rev. 118 (1960), p. 271.

[34] G. Charpak et al. "Measurement of the anomalous magnetic moment of the muon". In: Phys. Rev. Lett. 6 (1961), p. 128.

[35] G. Charpak et al. "A new measurement of the anomalous magnetic moment of the muon". In: Phys. Lett. 1 (1962), p. 16.

[36] J. Bailey et al. "Precision measurement of the anomalous magnetic moment of the muon". In: Phys. Lett. 28B (1968), p. 287.

[37] G. R. Henry, G. Schrank, and R. A. Swanson. "Measurement of the g-factor anomaly of the muon". In: Nuovo Cim. A63 (1969), p. 995.

[38] J. Bailey et al. (CERN III g-2 Collaboration). "g-2 experiment: measurement of the muon g value". In: Phys. Lett. 55B (1975), p. 420.

[39] J. Bailey et al. (CERN III g-2 Collaboration). "Final report on the CERN muon storage ring including the anomalous magnetic moment and the electric dipole moment of the muon, and a direct test of relativistic time dilation". In: Nucl. Phys. B150 (1979), pp. $1-75$.

[40] G. W. Bennett et al. (BNL g-2 Collaboration). "Improved measurement of the positive muon anomalous magnetic moment". In: Phys. Rev. D62 (2000), p. 091101.

[41] H. N. Brown et al. (BNL g-2 Collaboration). "Precise measurement of the positive muon anomalous magnetic moment". In: Phys. Rev. Lett. 86 (2001), p. 2227.

[42] G. W. Bennett et al. (BNL g-2 Collaboration). "Measurement of the positive muon anomalous magnetic moment to 0.7 ppm". In: Phys. Rev. Lett. 89 (2002), p. 101804.

[43] G. W. Bennett et al. (BNL g-2 Collaboration). "Measurement of the negative muon anomalous magnetic moment to 0.7 ppm". In: Phys. Rev. Lett. 92 (2004), p. 161802.

[44] G. W. Bennett et al. (BNL g-2 Collaboration). "Final report of the E821 muon anomalous magnetic moment measurement at BNL". In: Phys. Rev. D73 (2006), p. 072003.

[45] J. P. Miller, E. de Rafael, and B. L. Roberts. "Muon $(g-2)$ : Experiment and Theory". In: Rep. Prog. Phys. 70 (2007), p. 795. eprint: arXiv: 0703049 [hep-ph].

[46] T. D. Lee and C. N. Yang. "Question of parity conservation in weak interactions". In: Phys. Rev. 104 (1956), p. 254.

[47] C. S. Wu et al. "Experimental test of parity conservation in Beta decay". In: Phys. Rev. 105 (1957), p. 1413.

[48] J. Bailey et al. "The anomalous magnetic moment of positive and negative muons". In: Phys. Lett. 67B (1977), p. 225.

[49] F. J. M. Farley and E. Picaso. "The muon (g-2) experiments". In: Ann. Rev. Nucl. Part. Sci. 29 (1979), p. 243. 
[50] K. Ueno et al. (J-PARC muon g-2/EDM Collaboration). "Conceptual design of a radial vane silicon tracker for a new measurement of the muon anomalous magnetic moment $g-2$ and electric dipole moment at J-PARC". In: (2012). DOI: 10.1109/ NSSMIC . 2012.6551330.

[51] Y. Kondo et al. "Simulation study of muon acceleration using RFQ for a new muon g-2 experiment at J-PARC". In: IPAC-2015-THPF045 (2015).

[52] Y. Sato. "Muon g-2/EDM experiment at J-PARC". In: PoS KMI2017 (2017), p. 006.

[53] M. Aichter et al. "A multi-TeV linear collider based on CLIC technology". In: (2012). DOI: $10.5170 / C E R N-2012-007$.

[54] D. Stratakis et al. "Tapered channel for six-dimensional muon cooling towards micronscale emittances". In: Phys. Rev. ST Accel. Beams 16.9 (2013), p. 091001.

[55] D. Stratakis and R. B. Palmer. "Rectilinear six-dimensional ionization cooling channel for a muon collider: A theoretical and numerical study". In: Phys. Rev. ST Accel. Beams 18.3 (2015), p. 031003.

[56] S. J. Oliveros et al. "Exploration of a high luminosity $100 \mathrm{TeV}$ proton antiproton collider". In: (2017). eprint: arXiv: 1704.03891 [physics .acc-ph].

[57] P. M. McIntyre et al. "Collider in the sea: vision for a $500 \mathrm{TeV}$ world laboratory". In: (2017).

[58] C. Polly. "Muon g-2 science, management and publication plan". In: Fermilab E989 GM2-DocDB-8510 (2017).

[59] J. D. Jackson. Classical electrodynamics. 3rd ed. Wiley, 1999.

[60] K. A. Olive et al. (Particle Data Group). “ $\pi^{ \pm ” . ~ I n: ~ C h i n . ~ P h y s . ~ C 38 ~(2014), ~ p . ~} 090001$. URL: http://pdg. Ibl.gov.

[61] P. Renton. Electroweak interactions: an introduction to the physics of quarks and leptons. Cambridge University Press, 1990. DOI: 10.1017/CB09780511608148.

[62] J. Miller. "Expressions for $A(E)$ and $N(E)$ in the lab frame". In: BNL g-2 Internal Note 69 (1991).

[63] W. Liu et al. "High precision measurements of the ground state hyperfine structure interval of muonium and of the muon magnetic moment". In: Phys. Rev. Lett. 82 (1999), pp. 711-714.

[64] P. Winter. "Field overview". In: Fermilab E989 GM2-DocDB-9458 (2017).

[65] D. Still. "g-2 pulse train timing". In: Fermilab E989 GM2-DocDB-2395 (2014).

[66] D. W. Hertzog. "Next generation muon g-2 experiments". In: EPJ Web Conf. 118 (2016), p. 01015. DOI: 10 . 1051 / epjconf / 201611801015. eprint: arXiv : 1512 . 00928 [hep-ex].

[67] A. T. Fienberg et al. "Studies of an array of $\mathrm{PbF}_{2}$ Cherenkov crystals with large-area SiPM readout". In: Nucl. Instrum. Meth. A783 (2015), pp. 12-21.

[68] Y. K. Semertzidis et al. "The Brookhaven muon (g-2) storage ring high voltage quadrupoles". In: Nucl. Instrum. Meth. A503 (2003), pp. 458-484. 
[69] F. J. M. Farley. "Some calculations on resonance excitation with the g-2 ring". In: BNL Muon g-2 Note 106R (1992).

[70] W. T. Weng and S. R. Mane. "Fundamentals of particle beam dynamics and phase space". In: AIP Conf. Proc. 249 (1992), p. 3.

[71] J. Grange and W. Morse. "ESQ plate specs vs. n". In: Fermilab E989 GM2-DocDB3632 (2016).

[72] W. Wu. "The 3d E-field". In: Fermilab E989 GM2-DocDB-4688 (2016).

[73] Non-contact displacement Capacitec short form. http://www.process-controls . com/intertechnology/Capacitec/pdfs/ShortForm.pdf.

[74] W. Wu et al. "Using Capacitec to investigate quad plate alignment". In: Fermilab E989 GM2-DocDB-3709 (2016).

[75] J. Grange. "Top/bottom plate gap issue". In: Fermilab E989 UW-Elog-VacuumChamber85 (2016).

[76] W. Wu. "Quad plate alignment procedure". In: Fermilab E989 GM2-DocDB-3851 (2016).

[77] H. Friedsam. "Alignment results". In: Fermilab E989 GM2-DocDB-5955 (2017).

[78] W. Wu. "Q4 long quad alignment: laser scan data and hand tool data". In: Fermilab E989 UW-Elog-VacuumChamber-178 (2016).

[79] H. Nguyen. "Other options for Q1 outer qudrupole electrodes". In: Fermilab E989 GM2-DocDB-4169 (2016).

[80] V. Tishchenko and W. Wu. "Potting HV resistors". In: Fermilab E989 GM2-DocDB5236 (2017).

[81] V. Tishchenko et al. "Control electronics for high voltage pulsers conceptual design". In: Fermilab E989 GM2-DocDB-4436 (2016).

[82] D. A. Edwards and M. J. Syphers. An introduction to the physics of high energy accelerators. Wiley, 1993.

[83] M. Conte and W. W. Mackay. An introduction to the physics of particle accelerators. 2rd ed. World Scientific, 2008.

[84] OPERA simulation software. http://operafea.com.

[85] S. Redin. "Electric field in the muon g-2 storage ring: cylindrical correction". In: $B N L$ Muon g-2 Note 439 (2003).

[86] W. Wu et al. "Electrostatic focusing quadrupoles: plate alignment". In: Fermilab Muon g-2 E989 Note 116 (2017).

[87] S. R. Mane. "Solutions of Laplaces's equation and multipole expansions with a curved longitudinal axis". In: BNL Muon g-2 Note 91 (1991).

[88] M. Berz and K. Makino. "Quad fringe fields and tunes". In: Fermilab E989 GM2DocDB-7046 (2017). 
[89] W. Wu. "The 3D electric field map from OPERA-3D". In: Fermilab Muon g-2 E989 Note 117 (2017).

[90] A. T. Herrod et al. "Modeling of curvilinear electrostatic multipoles in the Fermilab muon g-2 storage ring". In: Fermilab E989 GM2-DocDB-6243 (2017).

[91] W. Wu and B. Quinn. "Beam dynamics in g-2 storage ring". In: IPAC-2017-MOPIK119 (2017). DOI: $10.18429 /$ JACoW-IPAC2017-MOPIK119.

[92] F. J. M. Farley. "Main formulas and numbers related to the g-2 beam and spin resonances". In: BNL Muon g-2 Note 396 (2001).

[93] M. Syphers. "CBO Tune Calculation". In: Fermilab E989 GM2-DocDB-10315 (2018).

[94] W. Wu and J. D. Crnkovic. "Quad scan study". In: Fermilab E989 GM2-DocDB-10027 (2018).

[95] J. D. Crnkovic and W. Wu. "Proposed 22 kV quad resonance scan". In: Fermilab E989 GM2-DocDB-9896 (2017).

[96] H. Jöstlein and P. Hattersley. "Notes on the CERN III fast rotation analysis". In: BNL Muon g-2 Note 271 (1971).

[97] A. Chapelain et al. "Intermediate report of the muon g-2 commissioning run task force". In: Fermilab E989 GM2-DocDB-7787 (2017).

[98] W. Wu. "Fast rotation analysis (on summer 2017 data)". In: Fermilab E989 GM2DocDB-9293 (2017).

[99] A. T. Fienberg. "T0 detector". In: Fermilab E989 GM2-DocDB-10911 (2018).

[100] W. Wu. "Fast rotation analysis: on FY18 commissioning data". In: Fermilab E989 GM2-DocDB-11052 (2018).

[101] J. Miller. "E-field and pitch corrections". In: Fermilab E989 GM2-DocDB-11082 (2018).

[102] E. D. Courant and H. S. Snyder. "Theory of the alternating gradient synchrotron". In: Annals Phys. 3 (1958), pp. 1-48. DOI: 10.1016/0003-4916(58)90012-5. 
LIST OF APPENDICES 
APPENDIX A: RESULTS OF QUAD PLATE ALIGNMENT 

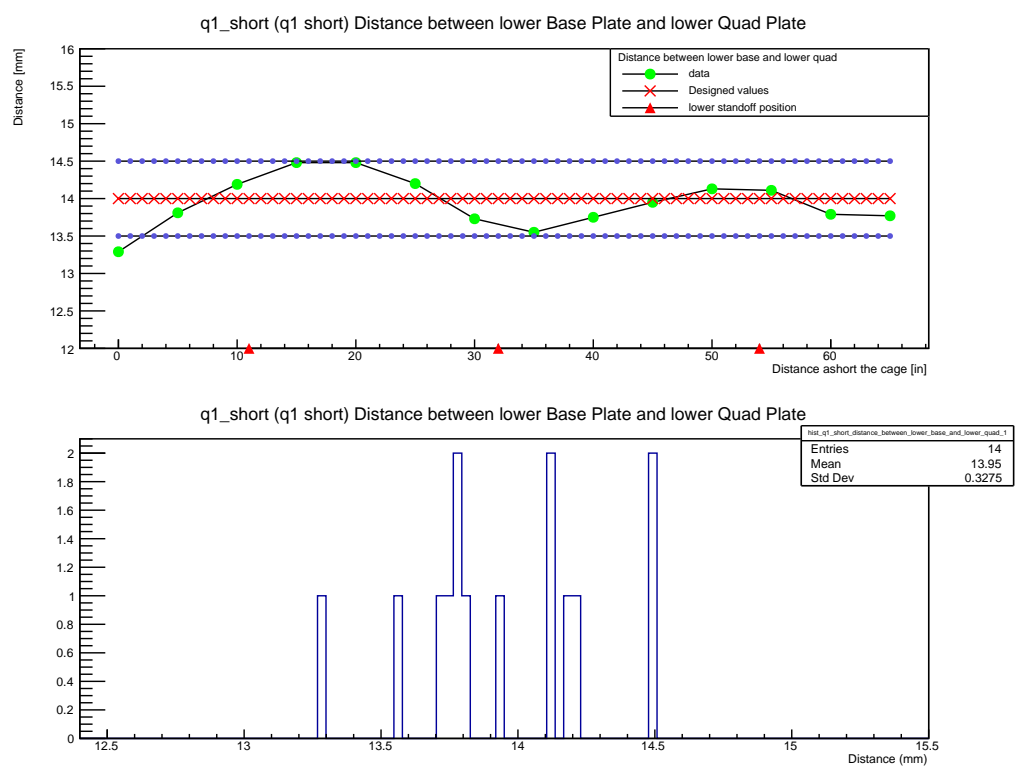

Figure A.1: Alignment result: Q1 short lower plate.
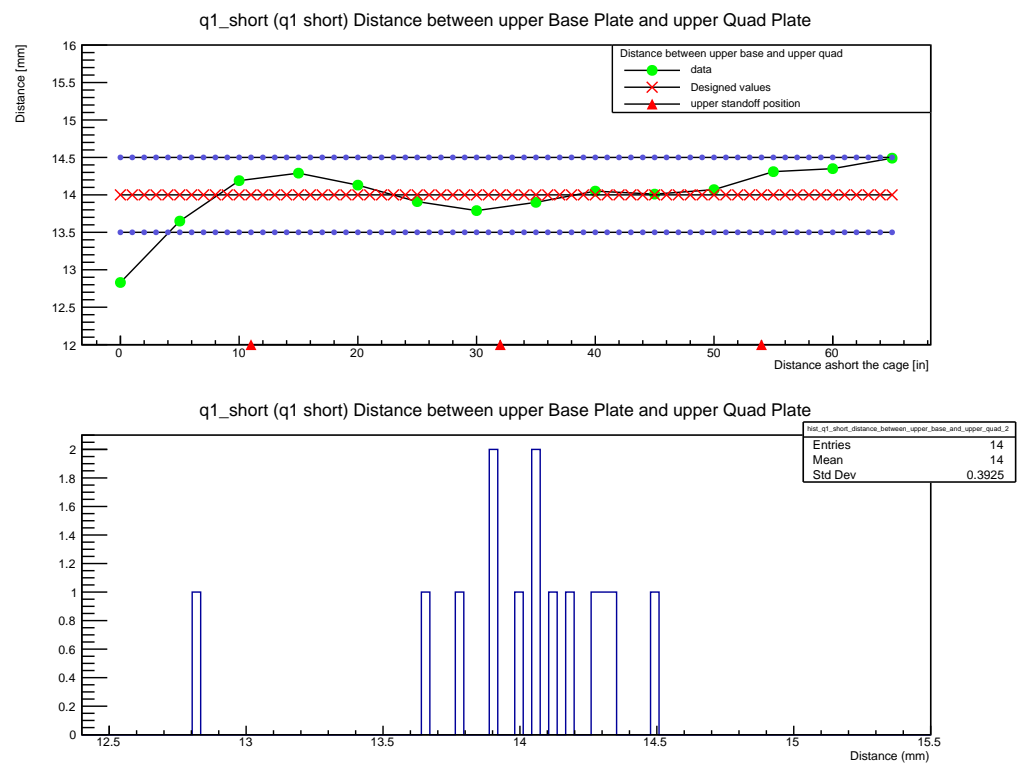

Figure A.2: Alignment result: Q1 short upper plate. 


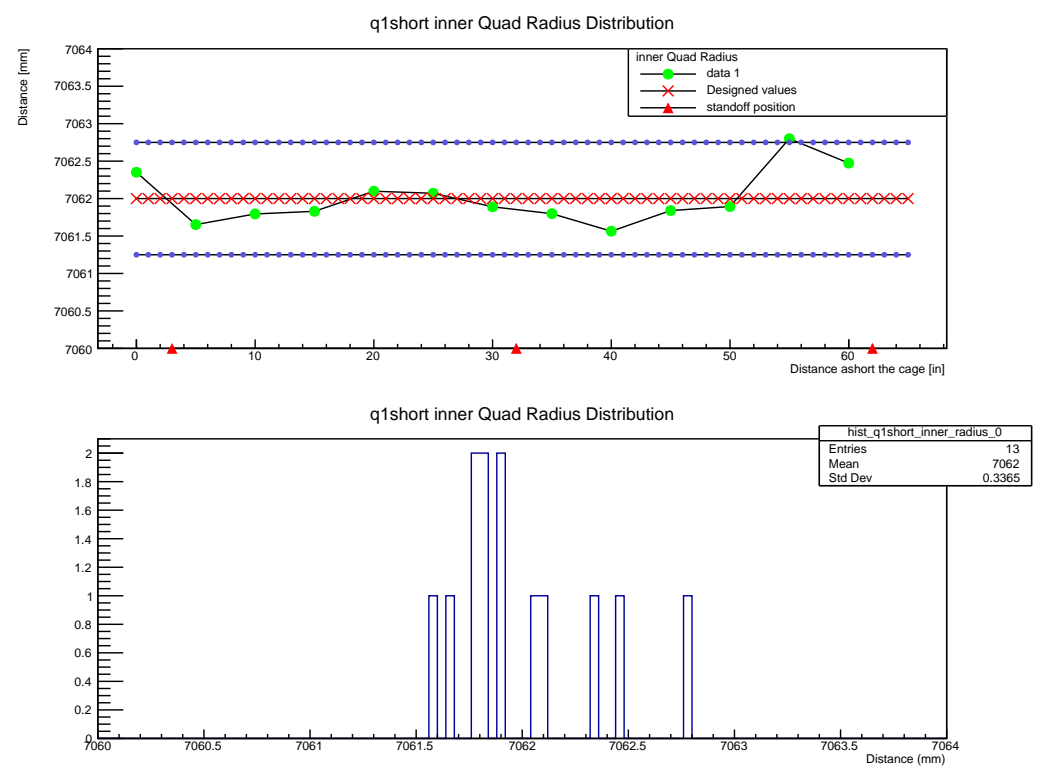

Figure A.3: Alignment result: Q1 short inner plate.

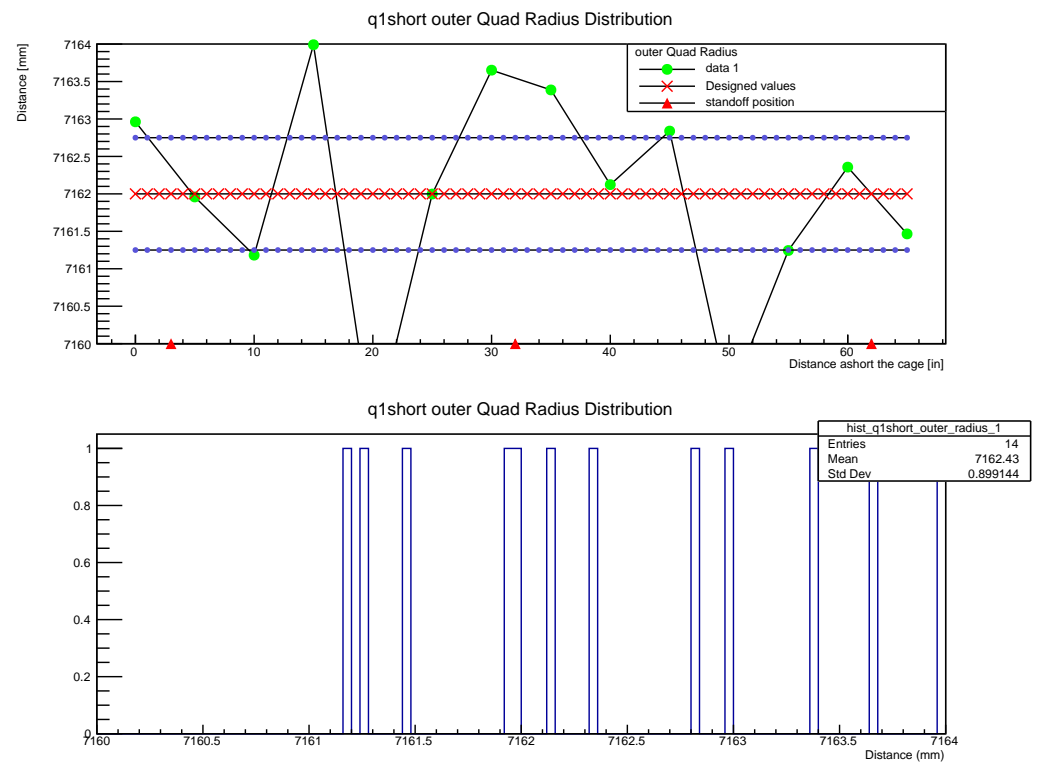

Figure A.4: Alignment result: Q1 short outer plate. 

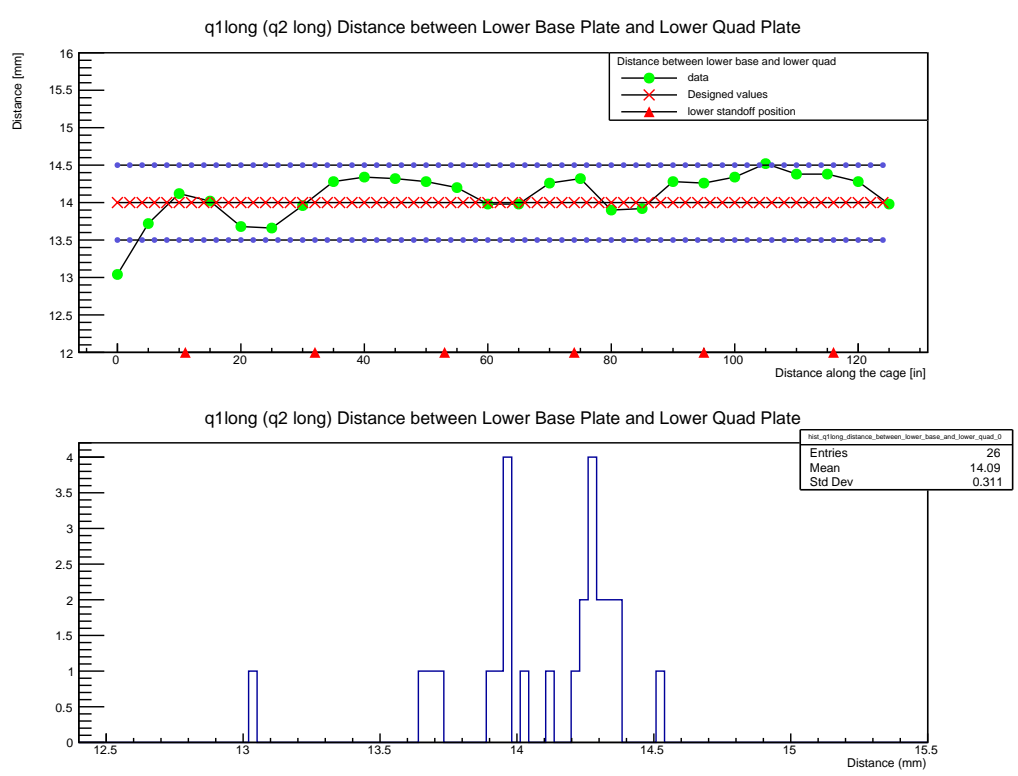

Figure A.5: Alignment result: Q1 long lower plate.

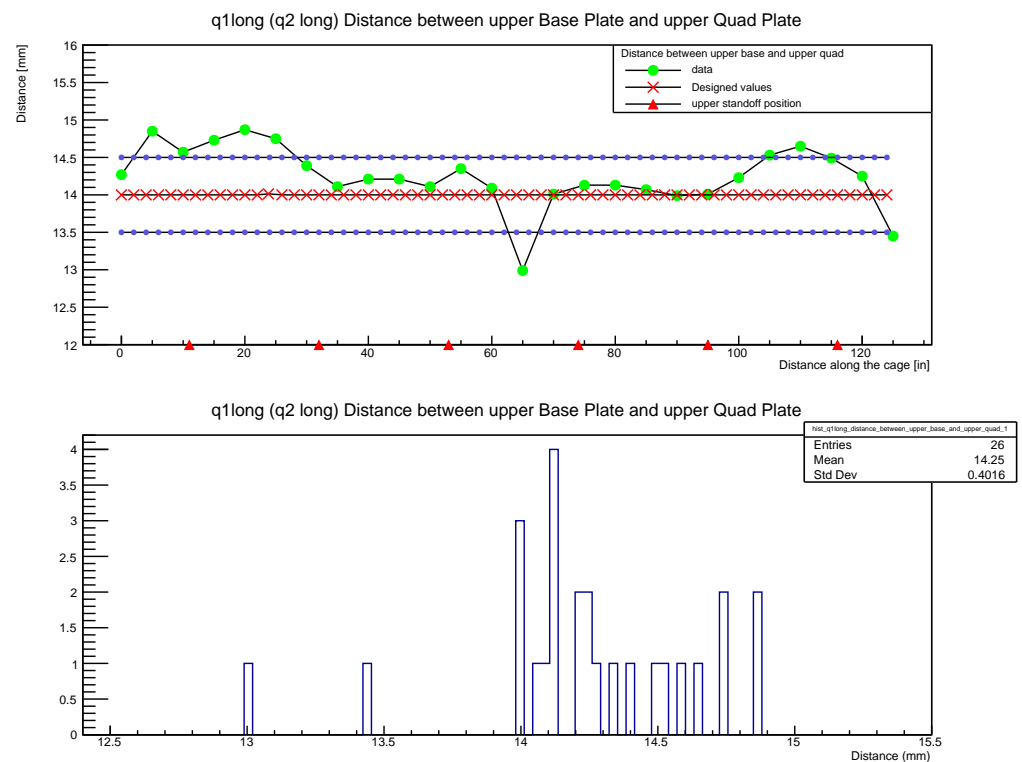

Figure A.6: Alignment result: Q1 long upper plate. 


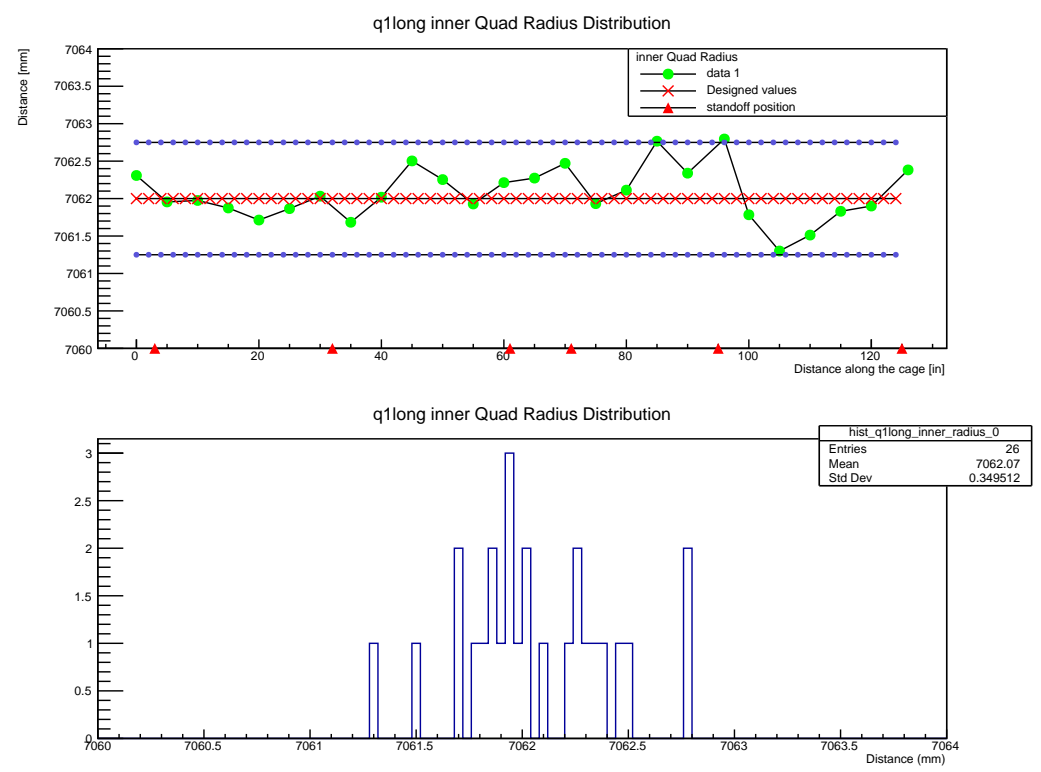

Figure A.7: Alignment result: Q1 long inner plate.

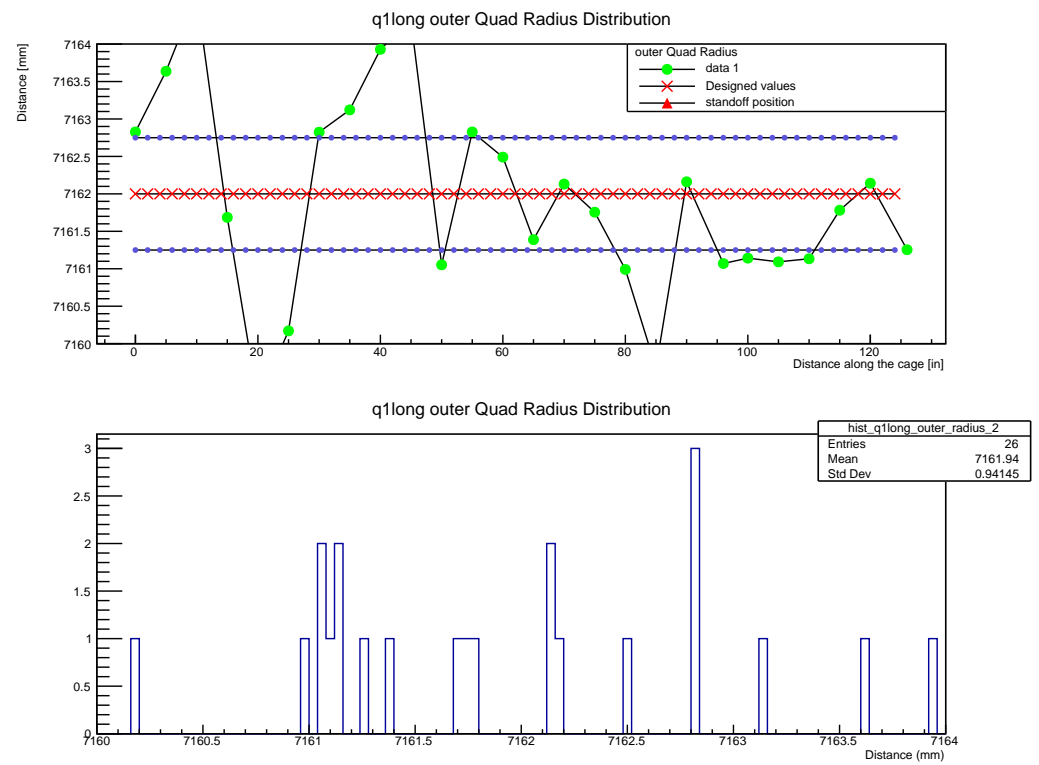

Figure A.8: Alignment result: Q1 long outer plate. 

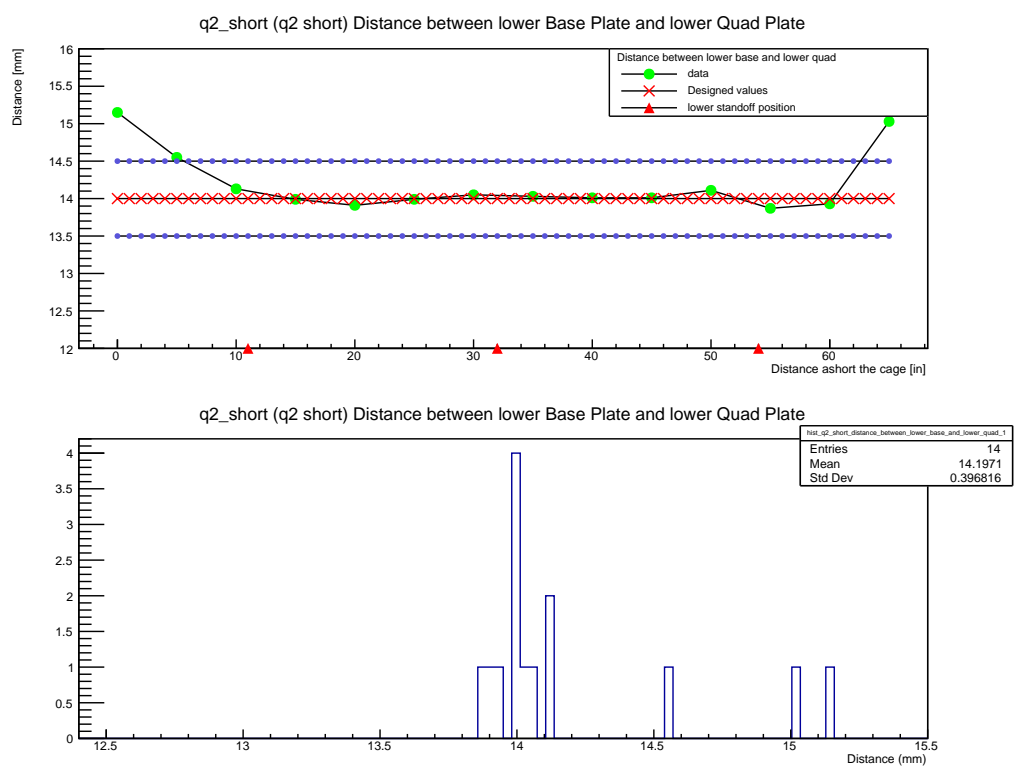

Figure A.9: Alignment result: Q2 short lower plate.

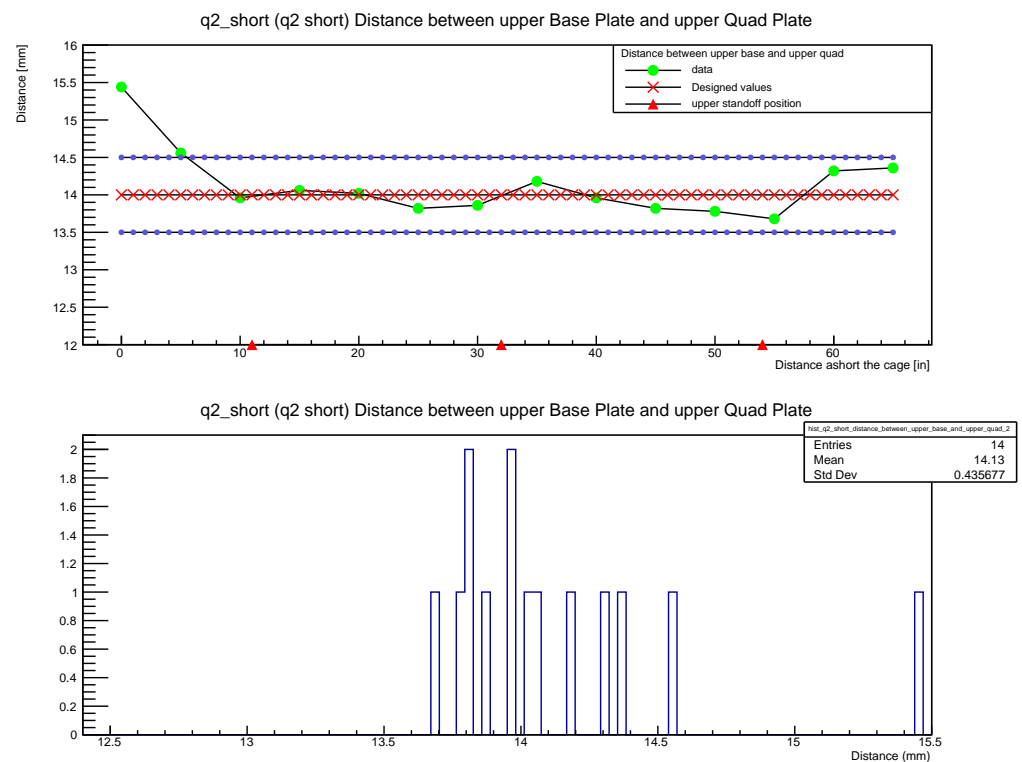

Figure A.10: Alignment result: Q2 short upper plate. 

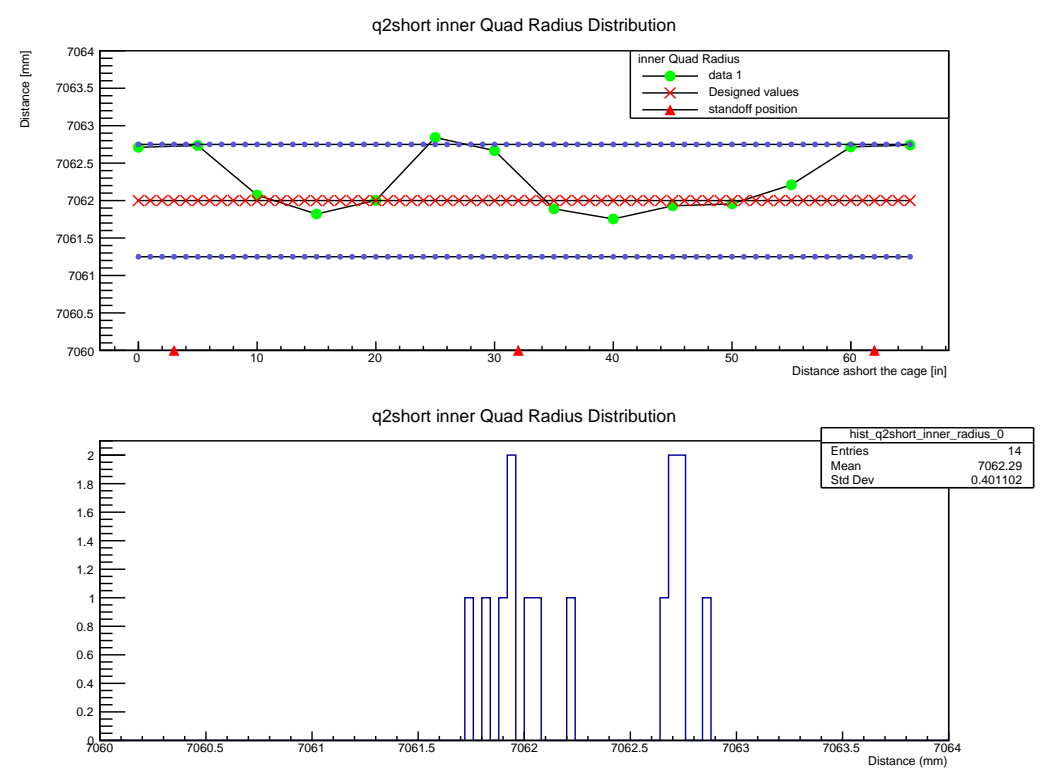

Figure A.11: Alignment result: Q2 short inner plate.

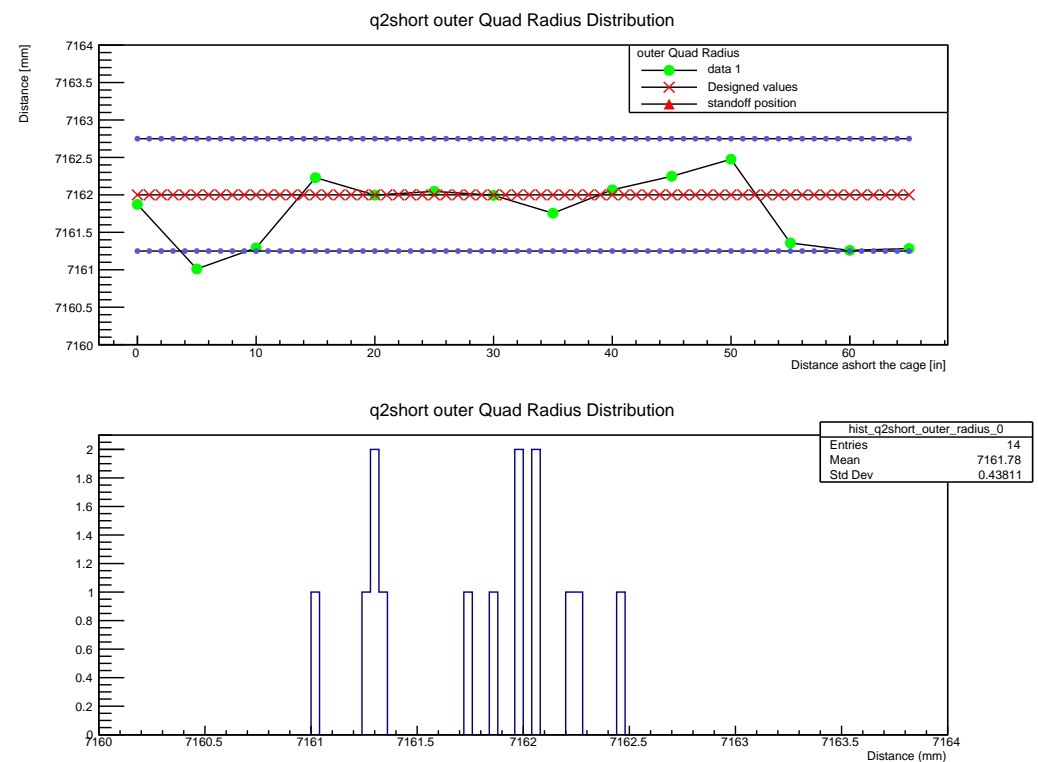

Figure A.12: Alignment result: Q2 short outer plate. 

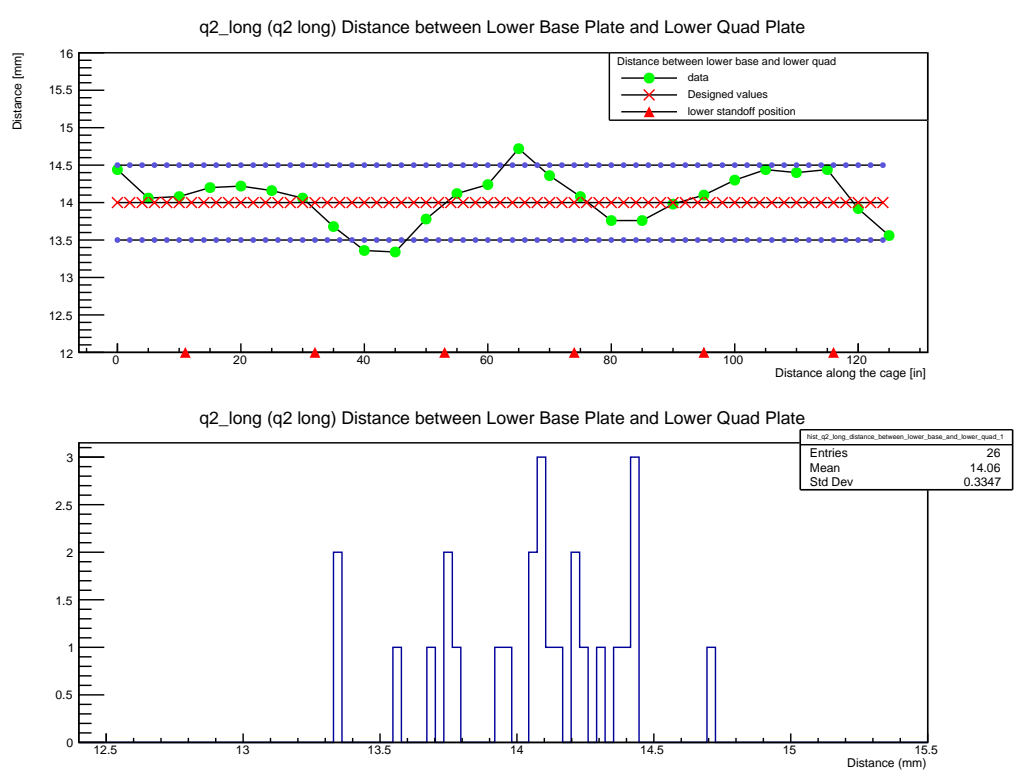

Figure A.13: Alignment result: Q2 long lower plate.

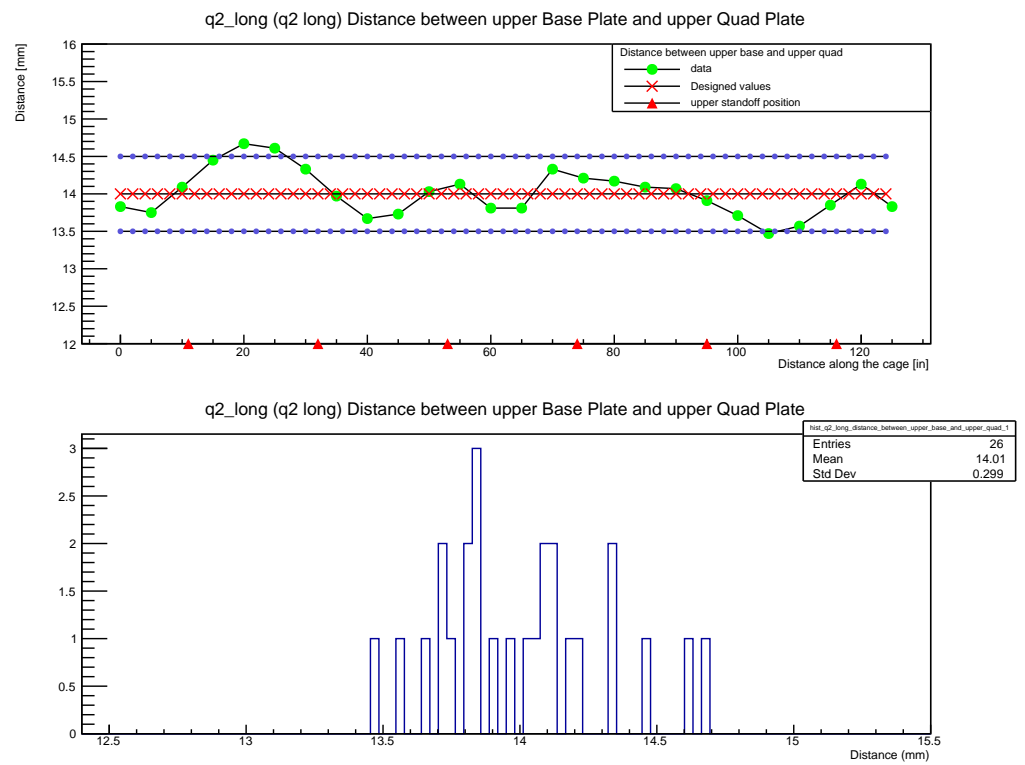

Figure A.14: Alignment result: Q2 long upper plate. 

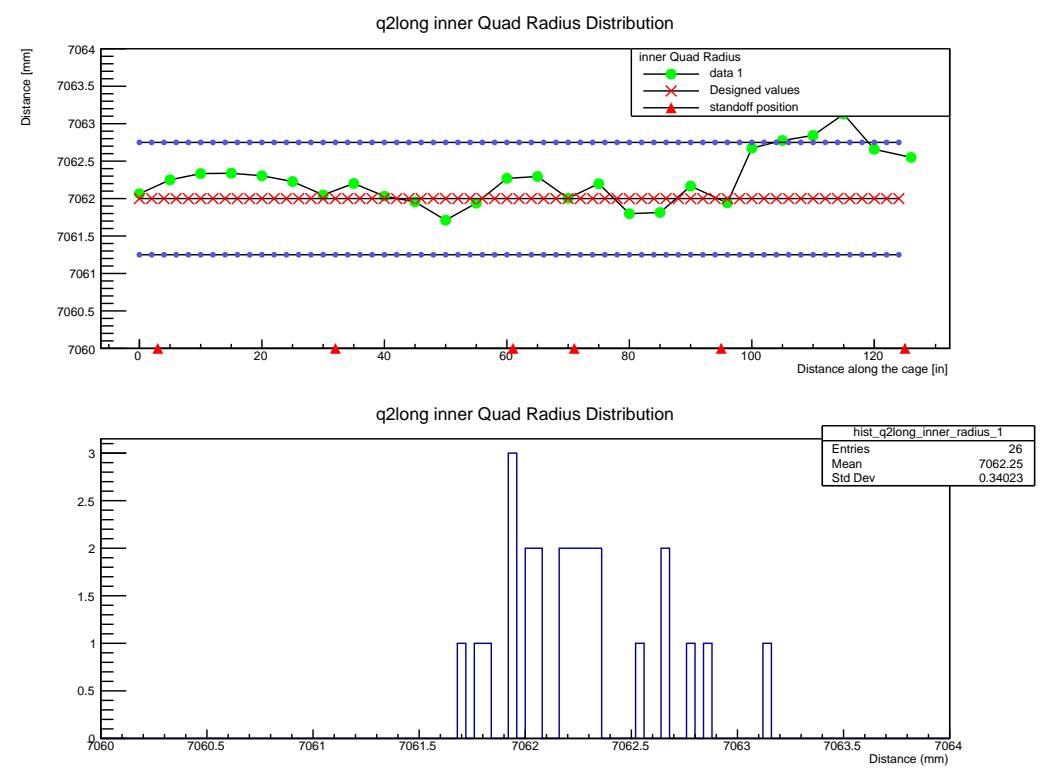

Figure A.15: Alignment result: Q2 long inner plate.

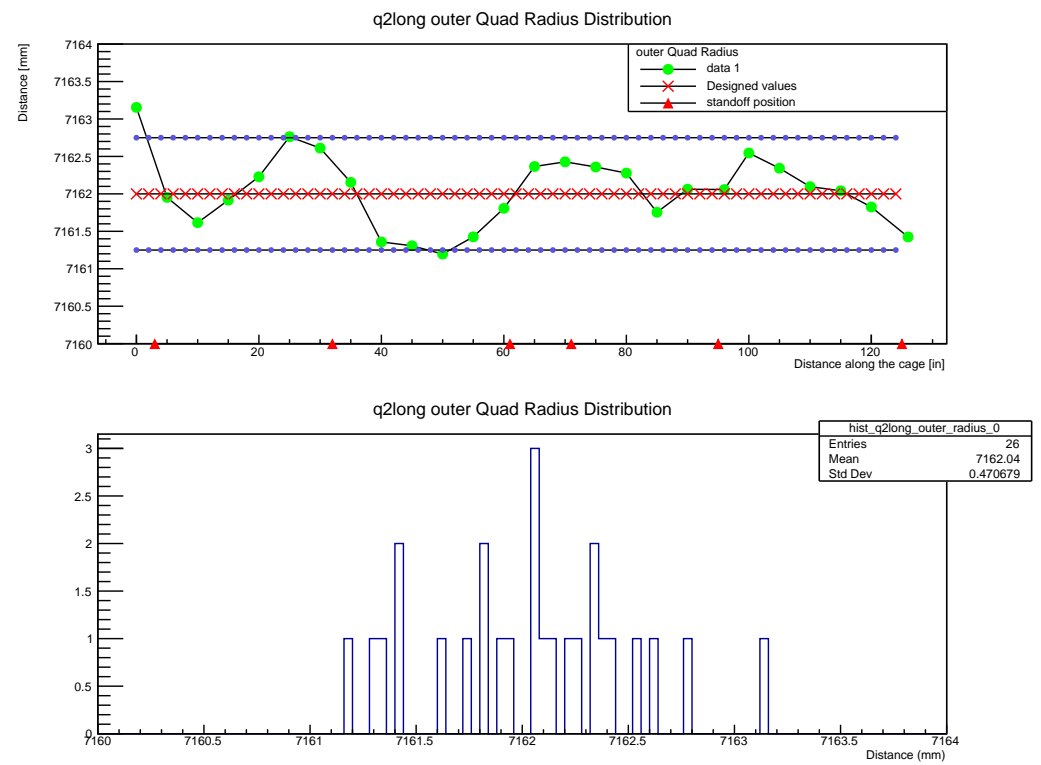

Figure A.16: Alignment result: Q2 long outer plate. 

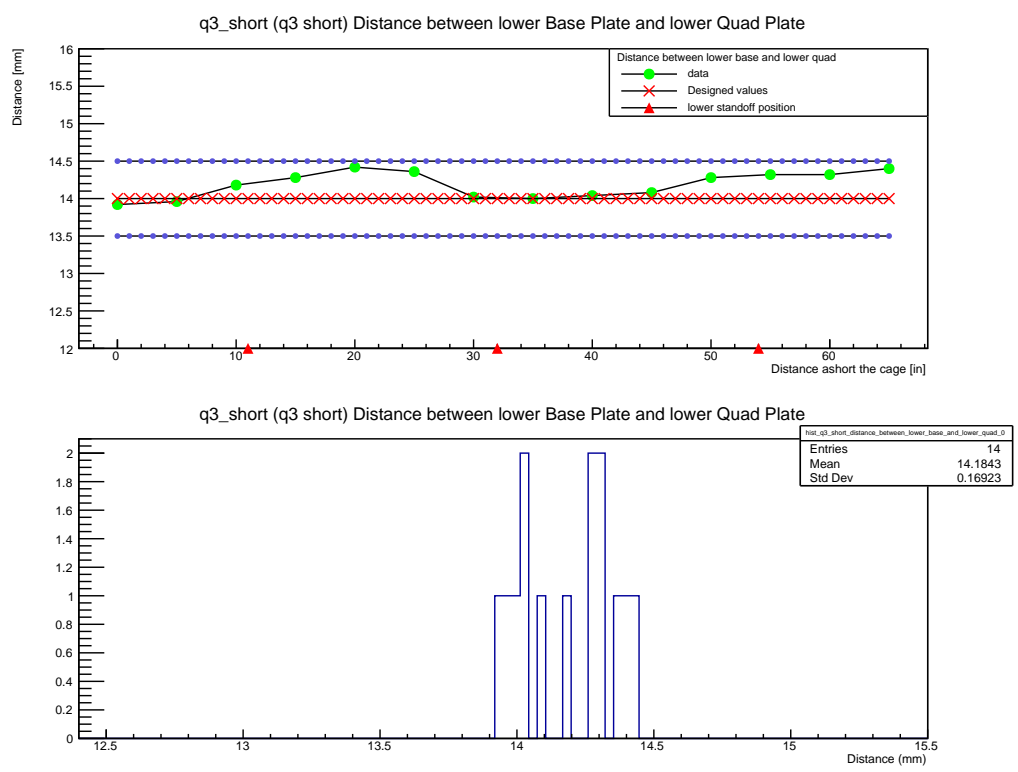

Figure A.17: Alignment result: Q3 short lower plate.
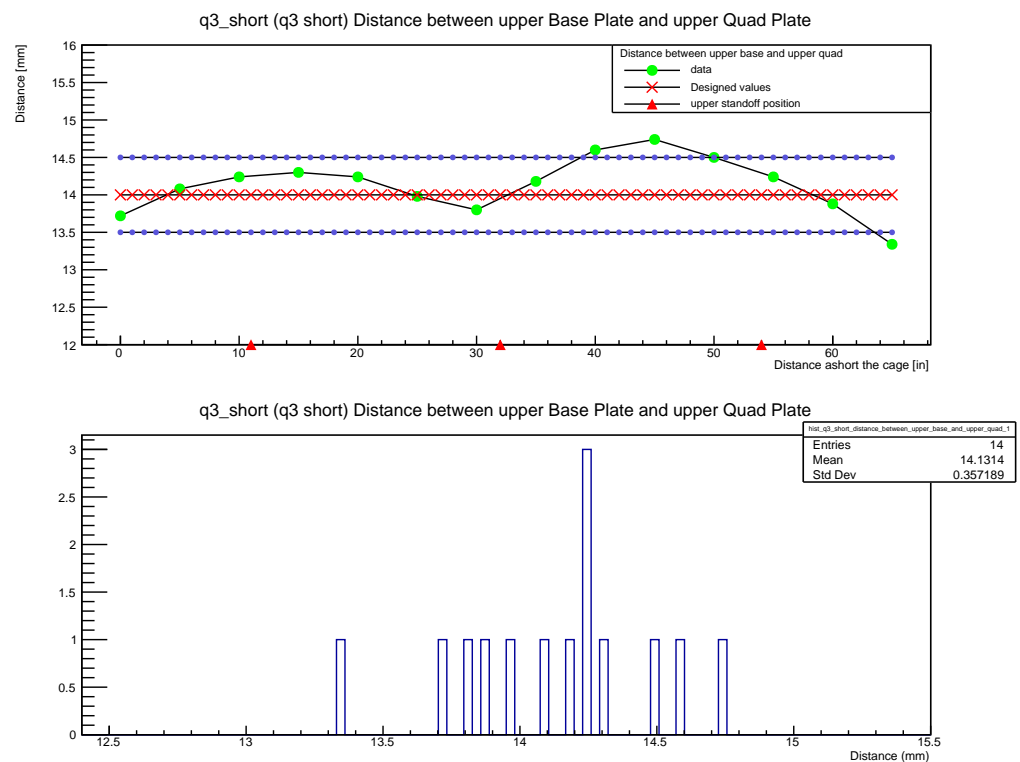

Figure A.18: Alignment result: Q3 short upper plate. 

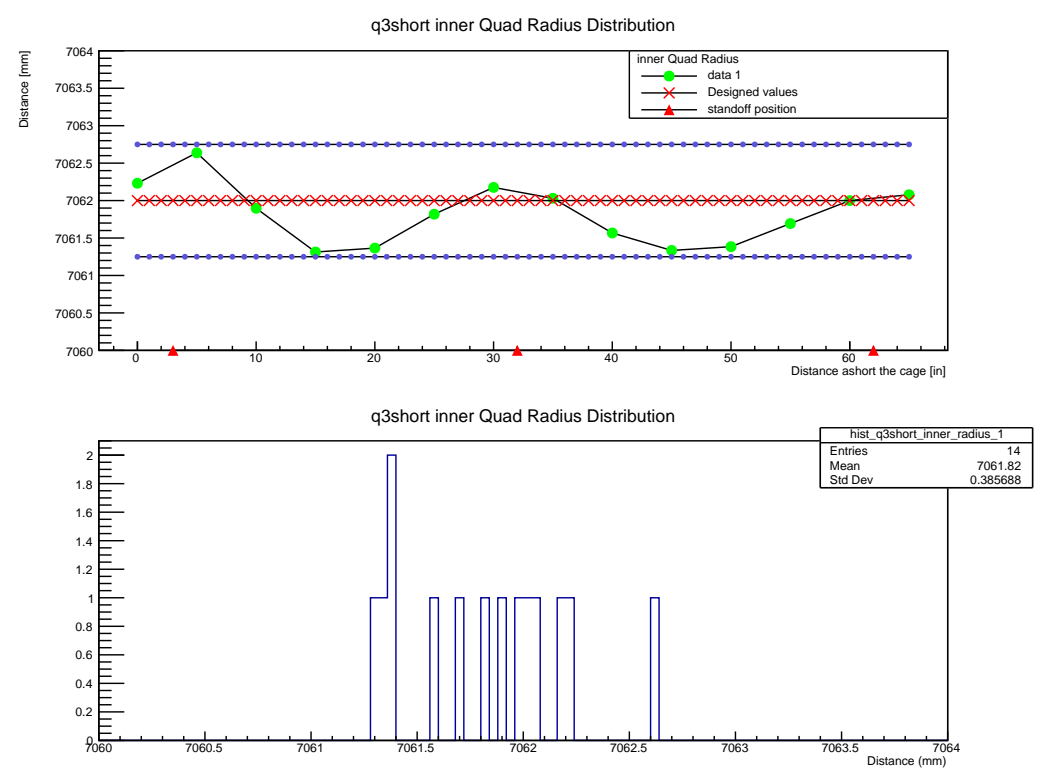

Figure A.19: Alignment result: Q3 short inner plate.

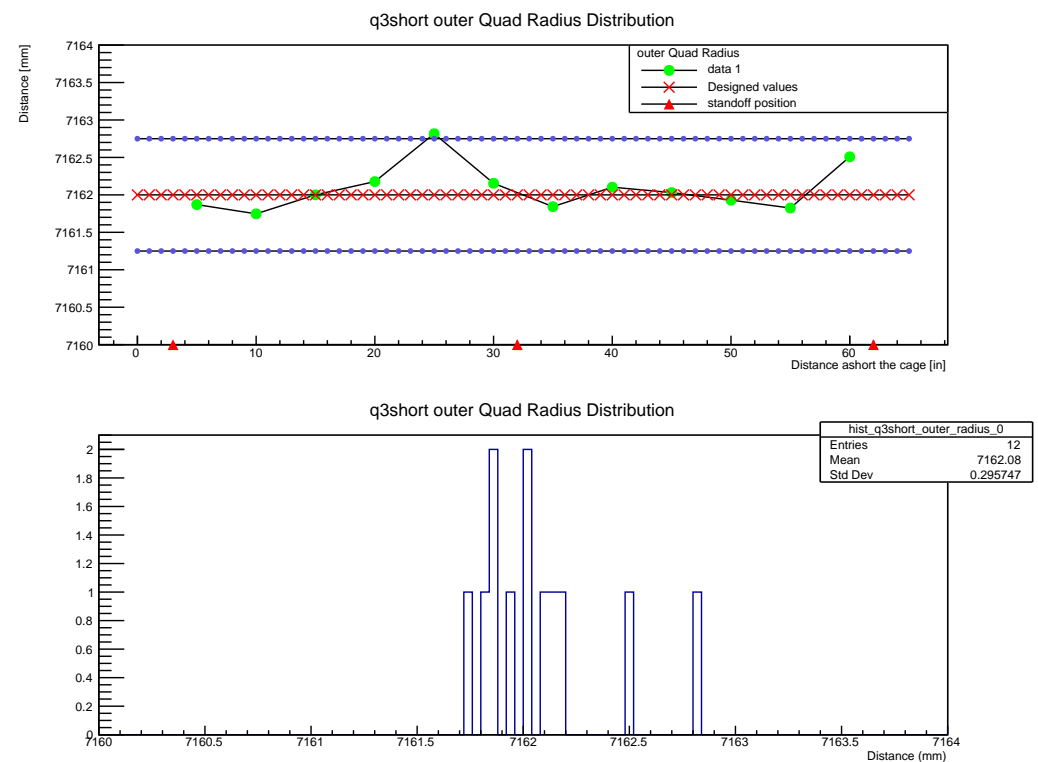

Figure A.20: Alignment result: Q3 short outer plate. 

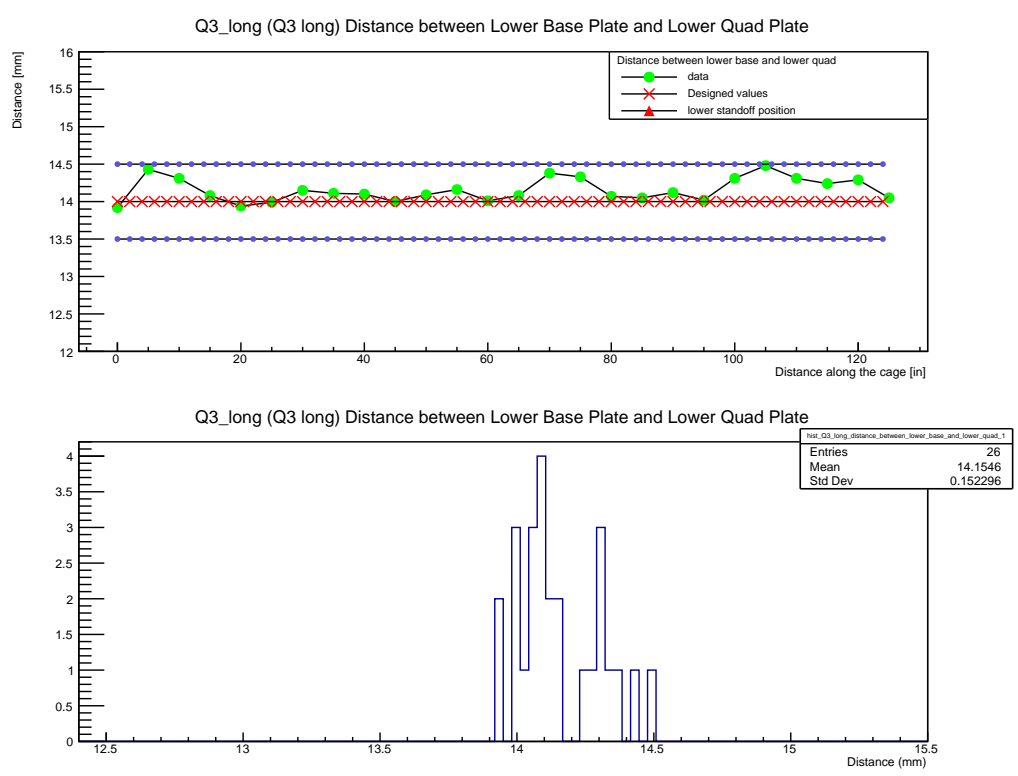

Figure A.21: Alignment result: Q3 long lower plate.
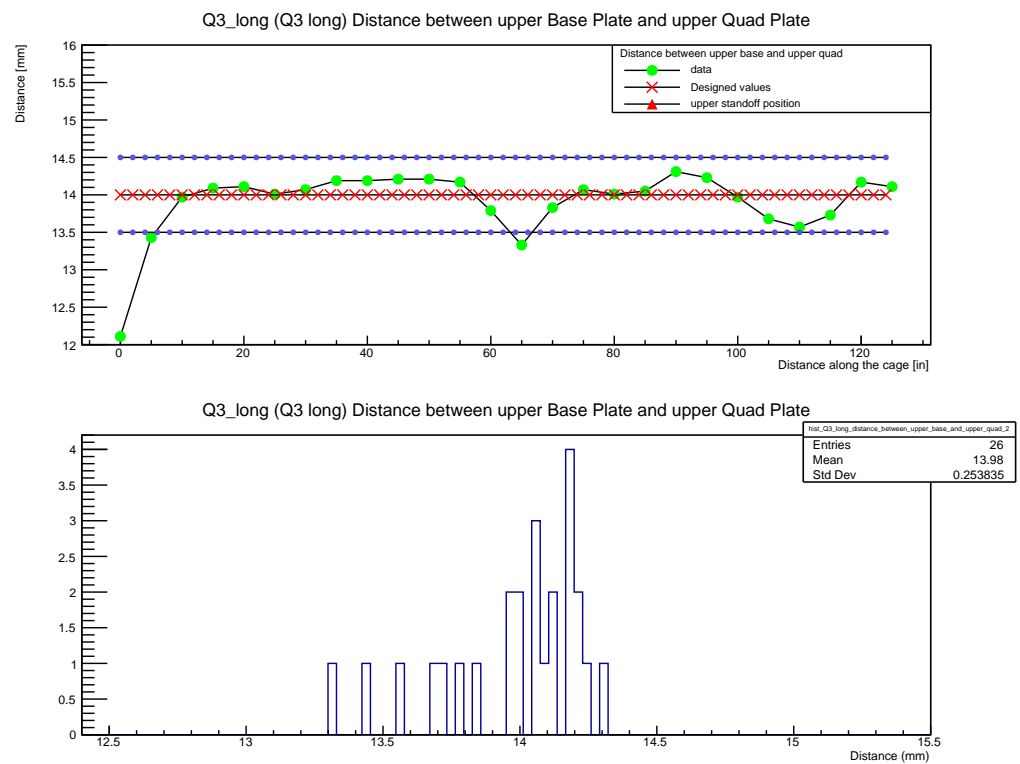

Figure A.22: Alignment result: Q3 long upper plate. 

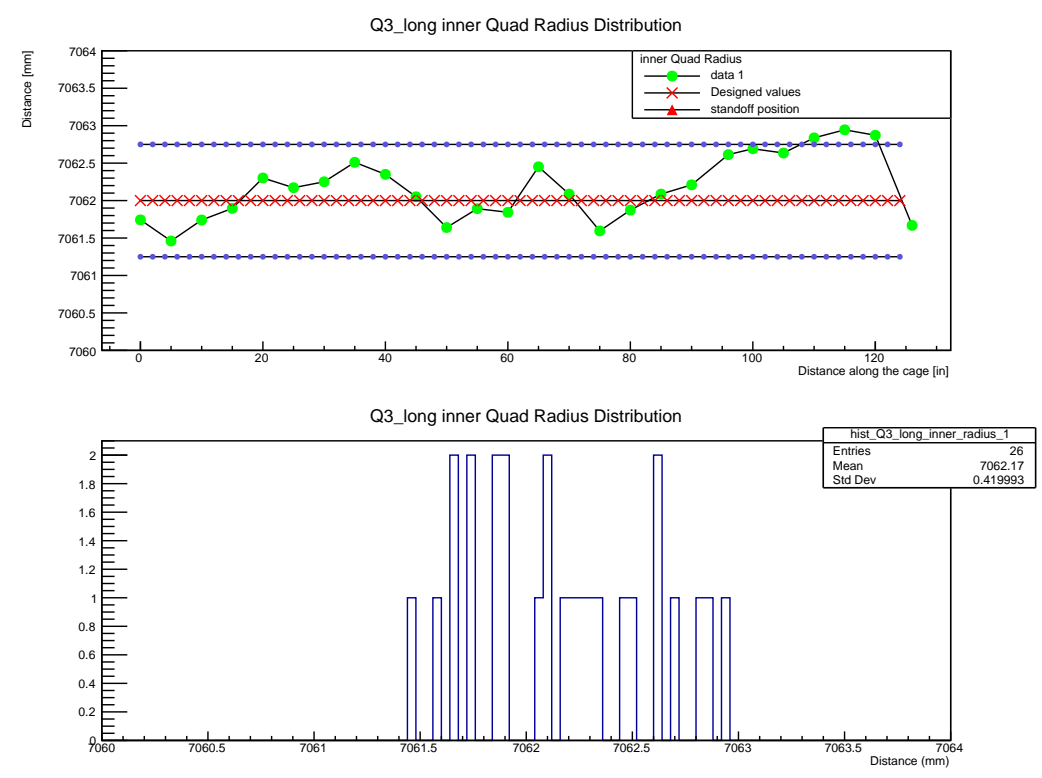

Figure A.23: Alignment result: Q3 long inner plate.

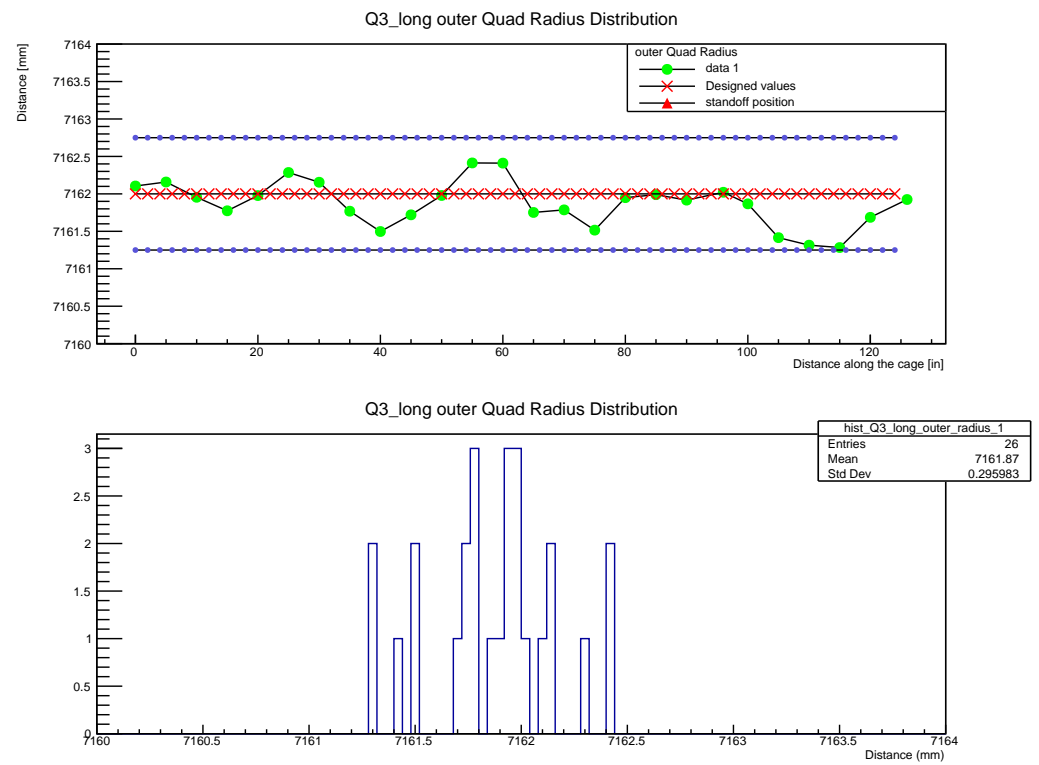

Figure A.24: Alignment result: Q3 long outer plate. 

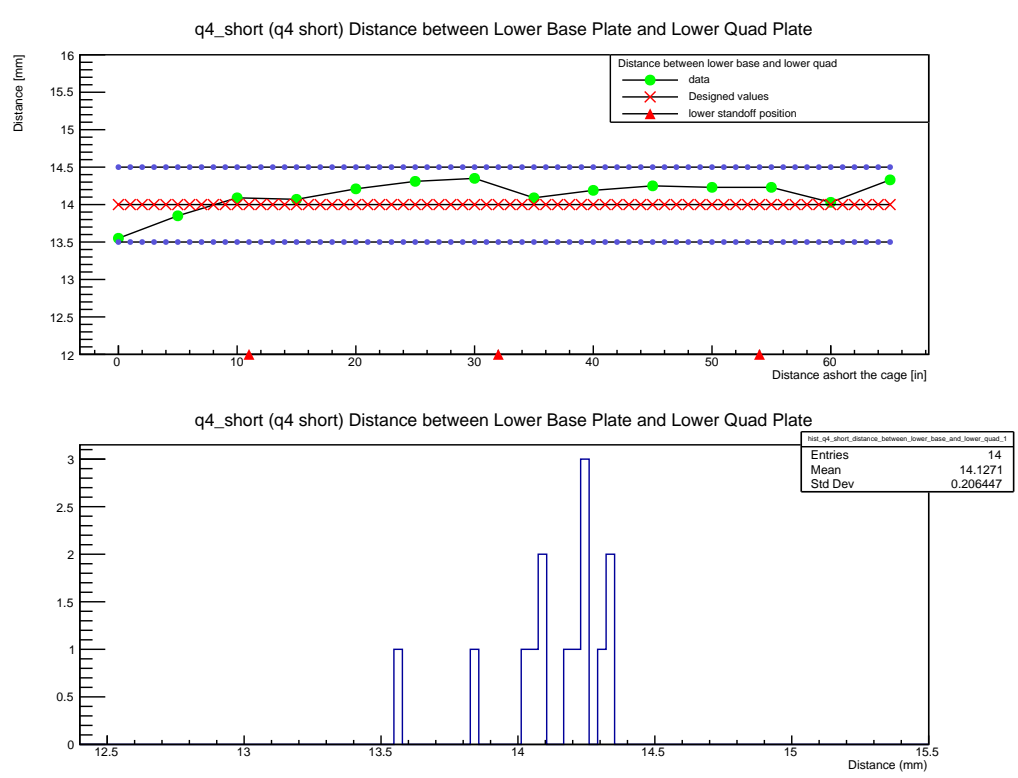

Figure A.25: Alignment result: Q4 short lower plate.

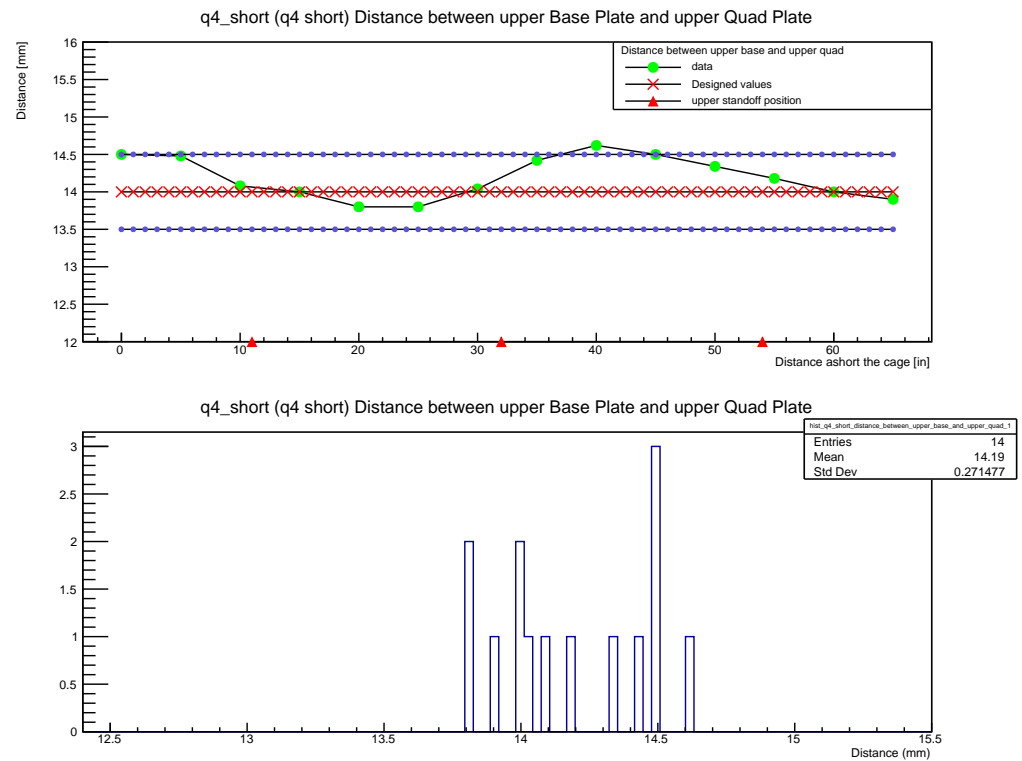

Figure A.26: Alignment result: Q4 short upper plate. 

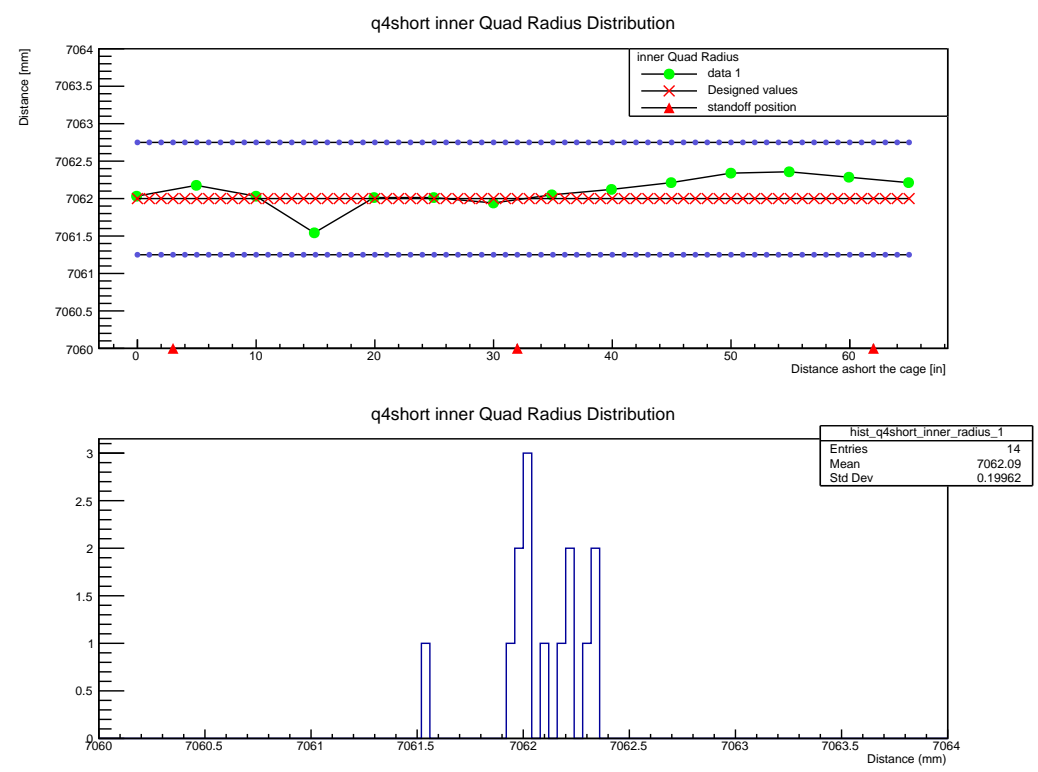

Figure A.27: Alignment result: Q4 short inner plate.

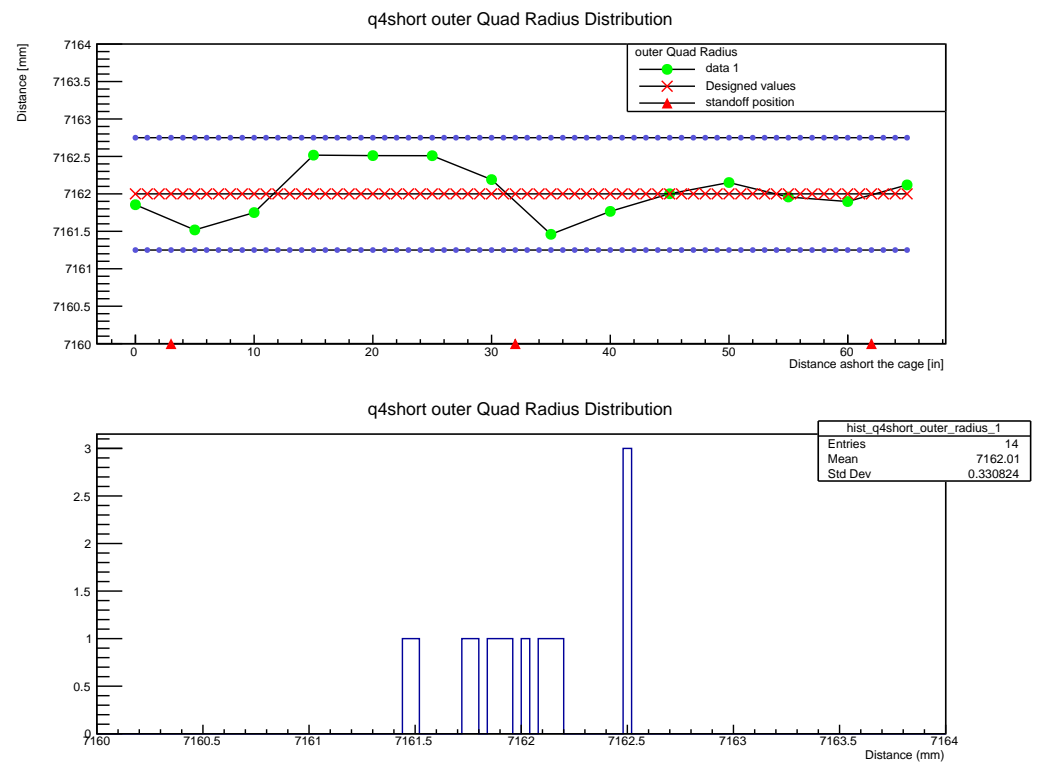

Figure A.28: Alignment result: Q4 short outer plate. 

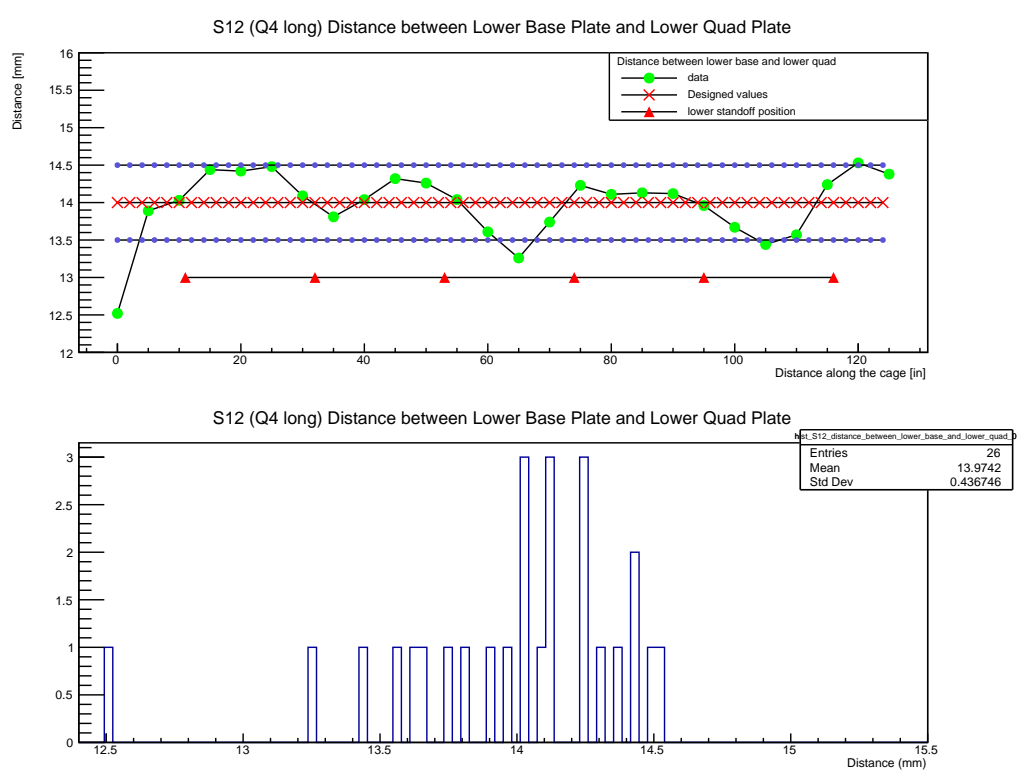

Figure A.29: Alignment result: Q4 long lower plate.

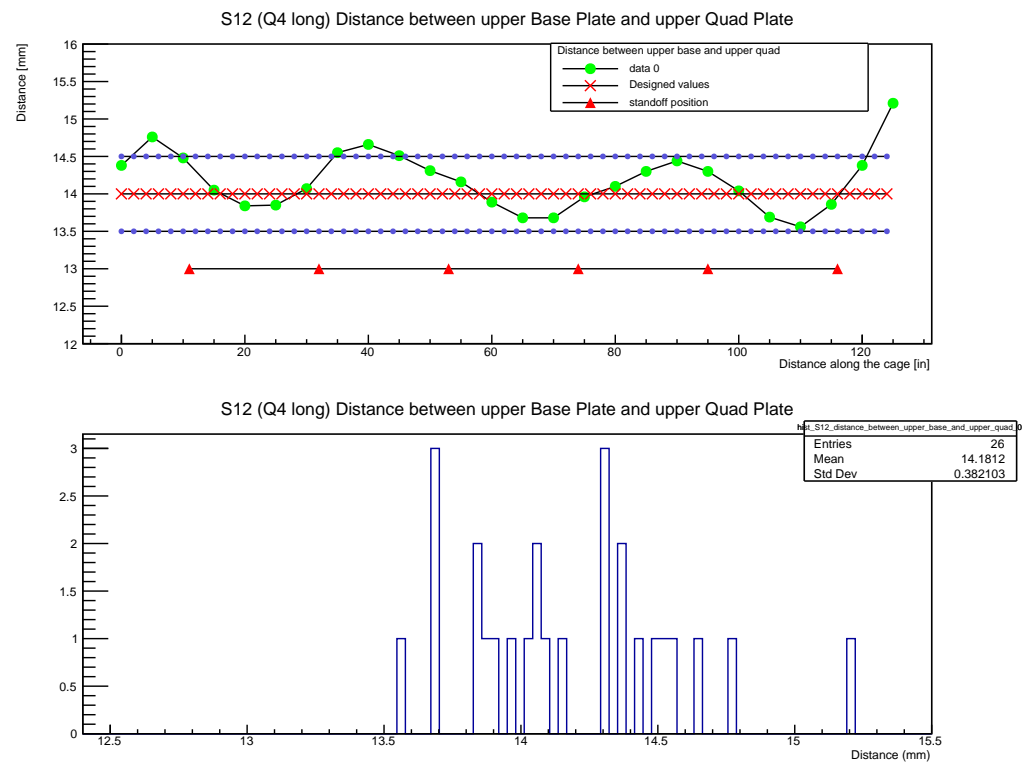

Figure A.30: Alignment result: Q4 long upper plate. 

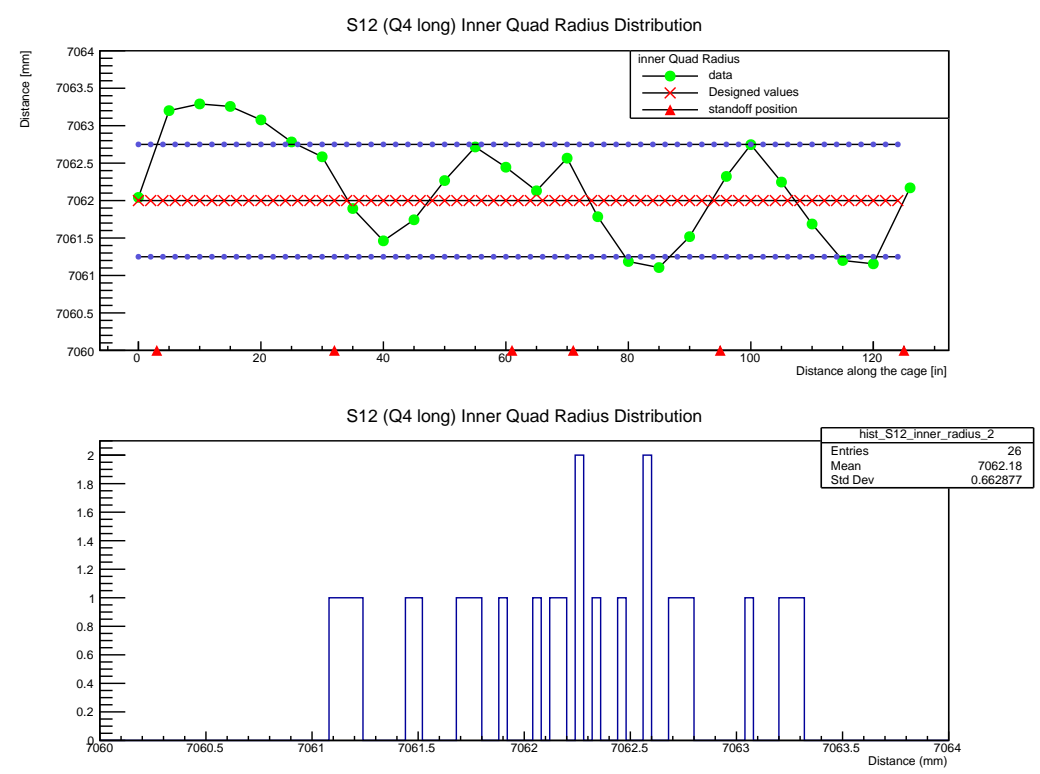

Figure A.31: Alignment result: Q4 long inner plate.

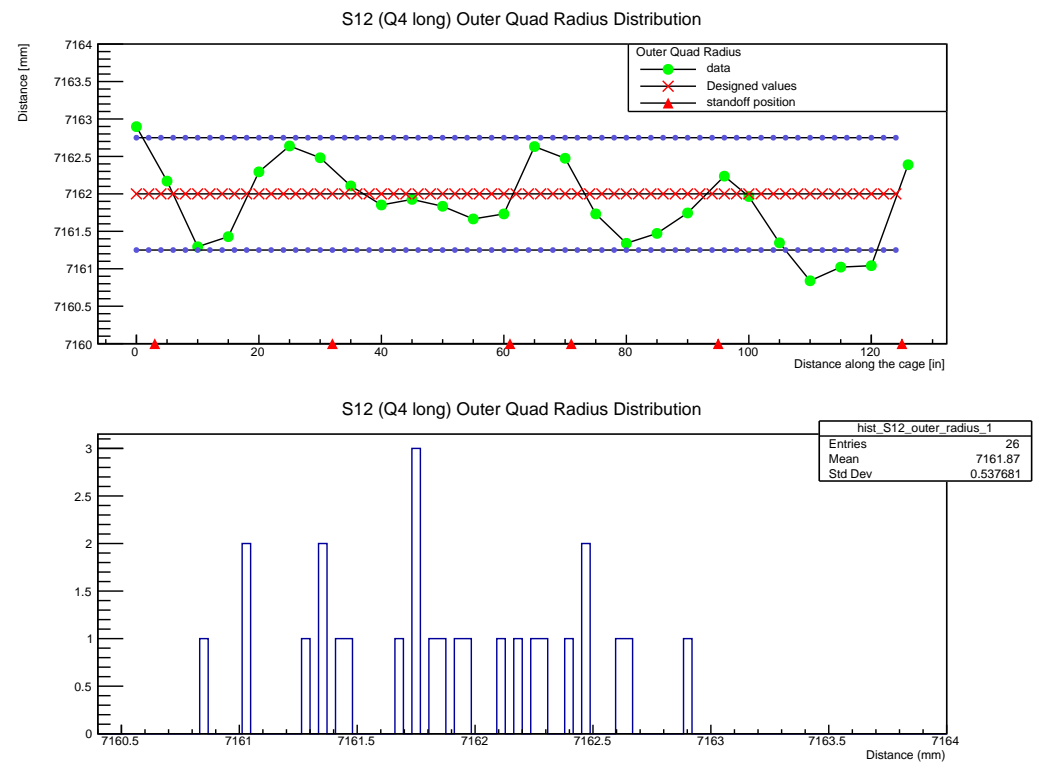

Figure A.32: Alignment result: Q4 long outer plate. 
APPENDIX B: BEAM DYNAMICS 


\section{B.1 Equation of Motion}

\section{B.1.1 Solution to Equation of Motion}

Eq. (4.13) and Eq. (4.18) are both of the form of Hill's Equation:

$$
x^{\prime \prime}+K(s) x=0
$$

where the "spring constant" $K$ is a function of position $s$. Here, we show some general ideas to obtain the solutions. We start with motion in $\hat{x}$-direction first. With the condition $0<n<1$, let

$$
x(s)=A \cos \frac{\sqrt{1-n} s}{\rho}+B \sin \frac{\sqrt{1-n} s}{\rho}
$$

then

$$
x^{\prime}(s)=\frac{d x}{d s}=\frac{\sqrt{1-n}}{\rho}\left(-A \sin \frac{\sqrt{1-n} s}{\rho}+B \cos \frac{\sqrt{1-n} s}{\rho}\right)
$$

Suppose we have $x(0)=x_{0}$ and $x^{\prime}(0)=x_{0}^{\prime}$, then the solutions are

$$
\begin{aligned}
x(s) & =\left(\cos \frac{\sqrt{1-n} s}{\rho}\right) x_{0}+\frac{\rho}{\sqrt{1-n}}\left(\sin \frac{\sqrt{1-n} s}{\rho}\right) x_{0}^{\prime} \\
x^{\prime}(s) & =-\frac{\sqrt{1-n}}{\rho}\left(\sin \frac{\sqrt{1-n} s}{\rho}\right) x_{0}+\left(\cos \frac{\sqrt{1-n} s}{\rho}\right) x_{0}^{\prime}
\end{aligned}
$$

or

$$
\left(\begin{array}{c}
x \\
x^{\prime}
\end{array}\right)=\left(\begin{array}{cc}
\cos \frac{\sqrt{1-n} s}{\rho} & \frac{\rho}{\sqrt{1-n}} \sin \frac{\sqrt{1-n} s}{\rho} \\
-\frac{\sqrt{1-n}}{\rho} \sin \frac{\sqrt{1-n} s}{\rho} & \cos \frac{\sqrt{1-n} s}{\rho}
\end{array}\right)\left(\begin{array}{c}
x_{0} \\
x_{0}^{\prime}
\end{array}\right)
$$


For motion with $\hat{y}$-direction, we only need replace $(1-n)$ with $n$ in Eq. (B.6). Therefore,

$$
\left(\begin{array}{c}
y \\
y^{\prime}
\end{array}\right)=\left(\begin{array}{cc}
\cos \frac{\sqrt{n} s}{\rho} & \frac{\rho}{\sqrt{n}} \sin \frac{\sqrt{n} s}{\rho} \\
-\frac{\sqrt{n}}{\rho} \sin \frac{\sqrt{n} s}{\rho} & \cos \frac{\sqrt{n} s}{\rho}
\end{array}\right)\left(\begin{array}{c}
y_{0} \\
y_{0}^{\prime}
\end{array}\right)
$$

\section{B.1.2 Courant-Snyder Parameters}

One very useful way is to rewrite the solutions to the equation of motion in the form of Courant-Snyder parameters: $\alpha(s), \beta(s)$ and $\gamma(s)$. For the details of the definition, please see

Ref. [82, 83, 102]. Here, we just show the idea of how to express the solution in the form of these parameters.

The general solution to the equation of motion can be written as

$$
x(s)=A \sqrt{\beta(s)} \cos [\psi(s)+\delta]
$$

The phase of the particle's oscillation advances through the period by an amount

$$
\psi\left(s_{0} \rightarrow s_{0}+C\right) \equiv \Delta \psi_{C}=\int_{s_{0}}^{s_{0}+C} \frac{d s}{\beta(s)}
$$

For a repeating period, we have

$$
\left(\begin{array}{c}
x \\
x^{\prime}
\end{array}\right)_{s_{0}+C}=\left(\begin{array}{cc}
\cos \Delta \psi_{c}+\alpha \sin \Delta \psi_{C} & \beta \sin \Delta \psi_{C} \\
-\gamma \sin \Delta \psi_{C} & \cos \Delta \psi_{c}-\alpha \sin \Delta \psi_{C}
\end{array}\right)\left(\begin{array}{c}
x \\
x^{\prime}
\end{array}\right)_{s_{0}}
$$

The transfer matrix in Eq. (B.10) can be written in a compact way as

$$
M=I \cos \Delta \psi_{c}+J \sin \Delta \psi_{C}
$$


with

$$
J \equiv\left(\begin{array}{cc}
\alpha & \beta \\
-\gamma & -\alpha
\end{array}\right)
$$

Suppose that the transfer matrix after multiplying all the individual matrices is given by

$$
M=\left(\begin{array}{ll}
a & b \\
c & d
\end{array}\right)
$$

then compare the two versions of $M$

$$
\left(\begin{array}{ll}
a & b \\
c & d
\end{array}\right)=\left(\begin{array}{cc}
\cos \Delta \psi_{c}+\alpha \sin \Delta \psi_{C} & \beta \sin \Delta \psi_{C} \\
-\gamma \sin \Delta \psi_{C} & \cos \Delta \psi_{c}-\alpha \sin \Delta \psi_{C}
\end{array}\right)
$$

from where we can find a way to calculate the phase advance and the Courant-Snyder parameters. For example,

$$
\begin{aligned}
\cos \Delta \psi_{C} & =\frac{1}{2}(a+d)=\frac{1}{2} \operatorname{Tr} M \\
\beta & =\frac{b}{\sin \Delta \psi_{C}}
\end{aligned}
$$

Another important parameter is the betatron tune $\nu$ (the number of oscillations per turn) in a circular machine. Here, we define

$$
\nu \equiv \frac{1}{2 \pi} \oint \frac{d s}{\beta(s)}
$$

We should also keep in mind that the phase advance between any two points now can be uniquely determined as

$$
\Delta \psi\left(s_{1} \rightarrow s_{2}\right)=\int_{s_{1}}^{s_{2}} \frac{d s}{\beta(s)}
$$




\section{B.2 Beam Dynamics in g-2 Storage Ring}

In this section, we are going to describe the beam dynamics in g-2 ring. Based on the lattice design of the g- 2 ring, we show the betatron oscillation relations. The general betatron oscillations can be described by [68]. Our basic solutions are:

$$
\begin{aligned}
x & =x_{e}+\sqrt{\epsilon_{x} \beta_{x}(s)} \cos \left[\nu_{x} \frac{s}{R}+\phi_{x}(s)\right] \\
y & =\sqrt{\epsilon_{y} \beta_{y}(s)} \cos \left[\nu_{y}+\phi_{y}(s)\right] \\
x_{e}(s) & =R_{e}(s)-R_{0}=D(s) \frac{p-p_{0}}{p}, \quad c p_{0}=e B R_{0}
\end{aligned}
$$

\section{B.2.1 Transfer Matrices}

As we show in Section B.1, the solutions to the equations of motion can be described by the transfer matrices.

In the $\hat{x}$-direction,

$$
M_{x n}=\left(\begin{array}{cc}
\cos \frac{\sqrt{1-n} s}{\rho} & \frac{\rho}{\sqrt{1-n}} \sin \frac{\sqrt{1-n} s}{\rho} \\
-\frac{\sqrt{1-n}}{\rho} \sin \frac{\sqrt{1-n} s}{\rho} & \cos \frac{\sqrt{1-n} s}{\rho}
\end{array}\right)
$$

For the regions without electric quadrupole $(n=0)$, the matrix becomes

$$
M_{x 0}=\left(\begin{array}{cc}
\cos \frac{s}{\rho} & \rho \sin \frac{s}{\rho} \\
-\frac{1}{\rho} \sin \frac{s}{\rho} & \cos \frac{s}{\rho}
\end{array}\right)
$$

In $\hat{y}$-direction,

$$
M_{y n}=\left(\begin{array}{cc}
\cos \frac{\sqrt{n} s}{\rho} & \frac{\rho}{\sqrt{n}} \sin \frac{\sqrt{n} s}{\rho} \\
-\frac{\sqrt{n}}{\rho} \sin \frac{\sqrt{n} s}{\rho} & \cos \frac{\sqrt{n} s}{\rho}
\end{array}\right)
$$


For the region without electric quadrupole $(n=0)$, the matrix becomes

$$
M_{y 0}=\left(\begin{array}{ll}
1 & s \\
0 & 1
\end{array}\right)
$$

Since there are four repeated periods in the whole ring, we can choose the initial phase by convenience and have any kind of lattice cell (one repeated period). For example, we consider a cell with a whole interval and a whole quadrupole. The transfer matrices of this cell can be calculated using

$$
\begin{aligned}
& M_{x}=M_{x n} \cdot M_{x 0}=\left(\begin{array}{cc}
\cos \frac{\sqrt{1-n} l_{q}}{\rho} & \frac{\rho}{\sqrt{1-n}} \sin \frac{\sqrt{1-n} l_{q}}{\rho} \\
-\frac{\sqrt{1-n}}{\rho} \sin \frac{\sqrt{1-n} l_{q}}{\rho} & \cos \frac{\sqrt{1-n} l_{q}}{\rho}
\end{array}\right)\left(\begin{array}{cc}
\cos \frac{l_{i}}{\rho} & \rho \sin \frac{l_{i}}{\rho} \\
-\frac{1}{\rho} \sin \frac{l_{i}}{\rho} & \cos \frac{l_{i}}{\rho}
\end{array}\right) \\
& =\left(\begin{array}{ccc}
\cos \frac{l_{i}}{\rho} \cos \frac{\sqrt{1-n} l_{q}}{\rho}-\frac{\rho}{\sqrt{1-n}} \sin \frac{l_{i}}{\rho} \sin \frac{\sqrt{1-n} l_{q}}{\rho} & \rho \sin \frac{l_{i}}{\rho} \cos \frac{\sqrt{1-n} l_{q}}{\rho}+\frac{\rho}{\sqrt{1-n}} \cos \frac{l_{i}}{\rho} \sin \frac{\sqrt{1-n} l_{q}}{\rho} \\
-\frac{1}{\rho} \sin \frac{l_{i}}{\rho} \cos \frac{\sqrt{1-n} l_{q}}{\rho}-\frac{\sqrt{1-n}}{\rho} \cos \frac{l_{i}}{\rho} \sin \frac{\sqrt{1-n} l_{q}}{\rho} & \cos \frac{l_{i}}{\rho} \cos \frac{\sqrt{1-n} l_{q}}{\rho}-\sqrt{1-n} \sin \frac{l_{i}}{\rho} \sin \frac{\sqrt{1-n} l_{q}}{\rho}
\end{array}\right)
\end{aligned}
$$

and

$$
M_{y}=M_{y n} \cdot M_{y 0}=\left(\begin{array}{cc}
\cos \frac{\sqrt{n} l_{q}}{\rho} & l_{i} \cos \frac{\sqrt{n} l_{q}}{\rho}+\frac{\rho}{\sqrt{n}} \sin \frac{\sqrt{n} l_{q}}{\rho} \\
-\frac{\sqrt{n}}{\rho} \sin \frac{\sqrt{n} l_{q}}{\rho} & \cos \frac{\sqrt{n} l_{q}}{\rho}-\frac{l_{i} \sqrt{n}}{\rho} \sin \frac{\sqrt{n} l_{q}}{\rho}
\end{array}\right)
$$

where $l_{i}$ is the interval length between quads and $l_{q}$ is the length of the quads.

First, we consider $\cos \Delta \psi_{c}=\cos \left(\pi \nu_{x, y} / 2\right)=\operatorname{Tr} M_{x, y} / 2$. Thus,

$$
\begin{gathered}
\cos \left(\pi \nu_{x} / 2\right)=\cos \frac{l_{i}}{\rho} \cos \frac{\sqrt{1-n} l_{q}}{\rho}-\frac{1}{2}\left(\sqrt{1-n}+\frac{1}{\sqrt{1-n}}\right) \sin \frac{l_{i}}{\rho} \sin \frac{\sqrt{1-n} l_{q}}{\rho} \\
\cos \left(\pi \nu_{y} / 2\right)=\cos \frac{\sqrt{n} l_{q}}{\rho}-\frac{\sqrt{n} l_{i}}{2 \rho} \sin \frac{\sqrt{n} l_{q}}{\rho}
\end{gathered}
$$




\section{B.2.2 Betatron Amplitudes}

To calculate the betatron amplitudes, we are going to use Eq. (B.16). We can choose any start position and consider the following cell. This gives us a way to calculate the betatron amplitude for any position. In fact, $\beta(s)$ is function with period equals $\pi \rho / 2$. In the g-2 ring, we require the beam will have the same shape in the phase space after a cell.

In g-2 ring, $\beta_{x}(s)$ is maximal in the middle of the intervals between quads and minimal in the middle of quads, while $\beta_{y}(s)$, inversely, is minimal in the middle of the intervals and maximal in the middle of quads.

Now, we consider a cell starting at the middle of the interval. The transfer matrices are

$$
\begin{aligned}
& M_{x i}=M_{x 0}\left(s=l_{i} / 2\right) \cdot M_{x n}\left(s=l_{q}\right) \cdot M_{x 0}\left(s=l_{i} / 2\right) \\
& =\left(\begin{array}{cc}
\cos ^{2} \frac{2}{2 \rho} \cos \frac{\sqrt{1-n} l_{q}}{2 \rho}-\sin ^{2} \frac{l_{i}}{2 \rho} \cos \frac{\sqrt{1-n} l_{q}}{\rho}+\frac{n-2}{2 \sqrt{1-n}} \sin \frac{l_{i}}{\rho} \sin \frac{\sqrt{1-n l_{q}}}{\rho} & \rho \sin \frac{l_{i}}{\rho} \cos \frac{\sqrt{1-n} l_{q}}{\rho}+\rho \frac{n-(n-2) \cos \frac{l_{i}}{\rho}}{2} \sin \frac{\sqrt{1-n l_{q}}}{\rho} \\
\frac{n+(n-2) \cos \frac{l_{i}}{\rho}}{2 \sqrt{1-n} \rho} \sin \frac{\sqrt{1-n} l_{q}}{\rho}-\frac{1}{\rho} \sin \frac{l_{i}}{\rho} \cos \frac{\sqrt{1-n} l_{q}}{\rho} & \cos ^{2} \frac{l_{i}}{2 \rho} \cos \frac{\sqrt{1-n} l_{q}}{\rho}-\sin ^{2} \frac{l_{i}}{2 \rho} \cos \frac{\sqrt{1-n} l_{q}}{\rho}+\frac{n-2}{2 \sqrt{1-n}} \sin \frac{l^{2}}{\rho} \sin \frac{\sqrt{1-n} l_{q}}{\rho}
\end{array}\right)
\end{aligned}
$$

and

$$
\begin{aligned}
M_{y i} & =M_{y 0}\left(s=l_{i} / 2\right) \cdot M_{y n}\left(s=l_{q}\right) \cdot M_{y 0}\left(s=l_{i} / 2\right) \\
& =\left(\begin{array}{cc}
\cos \frac{\sqrt{n} l_{q}}{\rho}-\frac{\sqrt{n} l_{i}}{2 \rho} \sin \frac{\sqrt{n} l_{q}}{\rho} & l_{i} \cos \frac{\sqrt{n} l_{q}}{\rho}+\frac{4 \rho^{2}-l_{i}^{2} n}{4 \sqrt{n} \rho} \sin \frac{\sqrt{n} l_{q}}{\rho} \\
-\frac{\sqrt{n}}{\rho} \sin \frac{\sqrt{n} l_{q}}{\rho} & \cos \frac{\sqrt{n} l_{q}}{\rho}-\frac{\sqrt{n} l_{i}}{2 \rho} \sin \frac{\sqrt{n} l_{q}}{\rho}
\end{array}\right)
\end{aligned}
$$


Therefore,

$$
\begin{aligned}
& \frac{\beta_{x, \max }}{\rho}=\frac{M_{x i}[1,2]}{\sin \left(\pi \nu_{x} / 2\right)}=\frac{\sin \frac{l_{i}}{\rho} \cos \frac{\sqrt{1-n} l_{q}}{\rho}+\frac{n-(n-2) \cos \frac{l_{i}}{\rho}}{2 \sqrt{1-n}} \sin \frac{\sqrt{1-n} l_{q}}{\rho}}{\sin \left(\pi \nu_{x} / 2\right)} \\
& =\frac{n \sin \frac{\sqrt{1-n} l_{q}}{\rho}}{2 \sqrt{1-n} \sin \left(\pi \nu_{x} / 2\right)}+\frac{2 \sqrt{1-n} \sin \frac{l_{i}}{\rho} \cos \frac{\sqrt{1-n} l_{q}}{\rho}-(n-2) \cos \frac{l_{i}}{\rho} \sin \frac{\sqrt{1-n} l_{q}}{\rho}}{2 \sqrt{1-n} \sin \left(\pi \nu_{x} / 2\right)} \\
& =\frac{n \sin \frac{\sqrt{1-n} l_{q}}{\rho}}{2 \sqrt{1-n} \sin \left(\pi \nu_{x} / 2\right)}+\frac{\sqrt{4(1-n) \sin ^{2} \frac{l_{i}}{\rho} \cos ^{2} \frac{\sqrt{1-n} l_{q}}{\rho}+(2-n)^{2} \cos ^{2} \frac{l_{i}}{\rho} \sin ^{2} \frac{\sqrt{1-n} l_{q}}{\rho} 4 \sqrt{1-n}(2-n) \cos \frac{l_{i}}{\rho} \cos \frac{\sqrt{1-n} l_{q}}{\rho} \sin \frac{l_{i}}{\rho} \sin \frac{\sqrt{1-n} l_{q}}{\rho}}}{2 \sqrt{1-n} \sin \left(\pi \nu_{x} / 2\right)} \\
& =\frac{n \sin \frac{\sqrt{1-n} l_{q}}{\rho}}{2 \sqrt{1-n} \sin \left(\pi \nu_{x} / 2\right)}+\sqrt{\frac{4(1-n) \cos ^{2} \frac{\sqrt{1-n} l_{q}}{\rho}+(2-n)^{2} \sin ^{2} \frac{\sqrt{1-n} l_{q}}{\rho}-4(1-n) \cos ^{2}\left(\pi \nu_{x} / 2\right)}{\left(2 \sqrt{1-n} \sin \left(\pi \nu_{x} / 2\right)\right)^{2}}} \\
& =\frac{n \sin \frac{\sqrt{1-n} l_{q}}{\rho}}{2 \sqrt{1-n} \sin \left(\pi \nu_{x} / 2\right)}+\sqrt{\left(\frac{n \sin \frac{\sqrt{1-n} l_{q}}{\rho}}{2 \sqrt{1-n} \sin \left(\pi \nu_{x} / 2\right)}\right)^{2}+1} \\
& \frac{\beta_{y, \min }}{\rho}=\frac{M_{y i}[1,2]}{\sin \left(\pi \nu_{y} / 2\right)}=\frac{4 l_{i} \sqrt{n} \rho \cos \frac{\sqrt{n} l_{q}}{\rho}+\left(4 \rho^{2}-l_{i}^{2} n\right) \sin \frac{\sqrt{n} l_{q}}{\rho}}{4 \sqrt{n} \rho^{2} \sin \left(\pi \nu_{y} / 2\right)} \\
& =\frac{4 l_{i} \sqrt{n} \rho \cos \frac{\sqrt{n} l_{q}}{\rho} \sin \frac{\sqrt{n} l_{q}}{\rho}+\left(4 \rho^{2}-l_{i}^{2} n\right) \sin ^{2} \frac{\sqrt{n} l_{q}}{\rho}}{4 \sqrt{n} \rho^{2} \sin \left(\pi \nu_{y} / 2\right) \sin \frac{\sqrt{n} l_{q}}{\rho}} \\
& =\frac{4 \rho^{2}-4 \rho^{2} \cos ^{2}\left(\pi \nu_{y} / 2\right)}{4 \sqrt{n} \rho^{2} \sin \left(\pi \nu_{y} / 2\right) \sin \frac{\sqrt{n} l_{q}}{\rho}}=\frac{\sin \left(\pi \nu_{y} / 2\right)}{\sqrt{n} \sin \frac{\sqrt{n} l_{q}}{\rho}}
\end{aligned}
$$

To calculate the $\beta_{x, \min }(s) / \rho$ and $\beta_{y, \max }(s) / \rho$, we consider a cell starting at the middle of a quad. The transfer matrices are

$$
\begin{aligned}
& M_{x q}=M_{x n}\left(s=l_{q} / 2\right) \cdot M_{x 0}\left(s=l_{i}\right) \cdot M_{x n}\left(s=l_{q} / 2\right) \\
& M_{y q}=M_{y n}\left(s=l_{q} / 2\right) \cdot M_{y 0}\left(s=l_{i}\right) \cdot M_{y n}\left(s=l_{q} / 2\right)
\end{aligned}
$$


Similarly, we have

$$
\begin{aligned}
& \frac{\beta_{x, \text { min }}}{\rho}=\frac{M_{x q}[1,2]}{\sin \left(\pi \nu_{x} / 2\right)} \\
& =\frac{-n \sin \frac{l_{i}}{\rho} \cos ^{2} \frac{\sqrt{1-n} l_{q}}{2 \rho}+\sqrt{1-n} \cos \frac{l_{i}}{\rho} \sin \frac{\sqrt{1-n} l_{q}}{\rho}+\sin \frac{l_{i}}{\rho} \cos \frac{\sqrt{1-n} l_{q}}{\rho}}{(1-n) \sin \left(\pi \nu_{x} / 2\right)} \\
& =-\frac{n}{2(1-n)} \frac{\sin \left(l_{i} / \rho\right)}{\sin \left(\pi \nu_{x} / 2\right)}+\sqrt{\left(\frac{n}{2(1-n)} \frac{\sin \left(l_{i} / \rho\right)}{\sin \left(\pi \nu_{x} / 2\right)}\right)^{2}+\frac{1}{1-n}} \\
& \frac{\beta_{y, \max }}{\rho}=\frac{M_{y q}[1,2]}{\sin \left(\pi \nu_{y} / 2\right)} \\
& =\frac{l_{i} \cos ^{2} \frac{\sqrt{n} l_{q}}{2 \rho}+\frac{2 \rho}{\sqrt{n}} \cos \frac{\sqrt{n} l_{q}}{2 \rho} \sin \frac{\sqrt{n} l_{q}}{2 \rho}}{\rho \sin \left(\pi \nu_{y} / 2\right)} \\
& =\frac{l_{i}}{2 \rho \sin \left(\pi \nu_{y} / 2\right)}+\sqrt{\left(\frac{l_{i}}{2 \rho \sin \left(\pi \nu_{y} / 2\right)}\right)^{2}+\frac{1}{n}}
\end{aligned}
$$

\section{B.2.3 Momentum Dispersion}

So far we have just considered monoenergetic particles, which have the same momentum as the ideal particle but differing transverse position and direction. In fact, there exists a momentum spread which implies a further radial broadening of the beam. The vertical oscillations will remain unaffected.

\section{Solution to equation of motion including momentum dispersion:}

We will start with the equation of motion:

$$
\frac{d^{2} x}{d s^{2}}-\frac{\rho+x}{\rho^{2}}=\frac{E_{x}-v_{z} B_{0}}{v_{z} B_{0} \rho}\left(1+\frac{x}{\rho}\right)^{2}
$$

Recall that we have used the relations $p=\gamma m v$ and $B_{0} \rho=p / e$ here. Therefore, we need to 
replace $p$ by $p+\delta p$ or add a factor of $p_{0} / p$ in Eq. (B.38). We will have

$$
\frac{d^{2} x}{d s^{2}}-\frac{\rho+x}{\rho^{2}}-\frac{E_{x}-v_{z} B_{0}}{v_{z} B_{0} \rho}\left(1+\frac{x}{\rho}\right)^{2}=-\frac{\Delta p}{p}\left[\frac{E_{x}-v_{z} B_{0}}{v_{z} B_{0} \rho}\left(1+\frac{x}{\rho}\right)^{2}\right]
$$

where $\Delta \equiv p-p_{0}$. The right side of Eq. (B.39) is exactly the same as before, and the right side of Eq. (B.39) can be approximately simplified as $\frac{1}{\rho} \frac{\Delta p}{p}$. Therefore, we obtain

$$
\frac{d^{2} x}{d \theta^{2}}+(1-n) x=\rho \frac{\Delta p}{p}
$$

The same discussion can be also applied to the y-component of equation of motion. With our assumption and approximation, it is the same.

If we write the closed orbit of an off-momentum (momentum differs from the ideal momentum) particle in the form

$$
x=D(p, s) \frac{\Delta p}{p}
$$

the closed solution requires that

$$
D(p, s+C)=D(p, s)
$$

Some discussions about how to achieve this solution are included in Ref. [82, 83]. Here, we just present a general procedure to reach our goal as before.

The solution of Eq. (B.40) can be written as

$$
x(s)=A \cos \frac{\sqrt{1-n} s}{\rho}+B \sin \frac{\sqrt{1-n} s}{\rho}+\frac{\rho}{1-n} \frac{\Delta p}{p}
$$

Also,

$$
x^{\prime}(s)=\frac{d x}{d s}=\frac{\sqrt{1-n}}{\rho}\left(-A \sin \frac{\sqrt{1-n} s}{\rho}+B \cos \frac{\sqrt{1-n} s}{\rho}\right)
$$


For $x(0)=x_{0}$ and $x^{\prime}(0)=x_{0}^{\prime}$, then these become

$$
\begin{aligned}
x(s)=\quad & \left(\cos \frac{\sqrt{1-n} s}{\rho}\right) x_{0}+\frac{\rho}{\sqrt{1-n}}\left(\sin \frac{\sqrt{1-n} s}{\rho}\right) x_{0}^{\prime} \\
& +\frac{\rho}{1-n}\left(1-\cos \frac{\sqrt{1-n} s}{\rho}\right)\left(\frac{\Delta p}{p}\right)_{0} \\
x^{\prime}(s)=\quad & -\frac{\sqrt{1-n}}{\rho}\left(\sin \frac{\sqrt{1-n} s}{\rho}\right) x_{0}+\left(\cos \frac{\sqrt{1-n} s}{\rho}\right) x_{0}^{\prime} \\
& +\left(\frac{1}{\sqrt{1-n}} \sin \frac{\sqrt{1-n} s}{\rho}\right)\left(\frac{\Delta p}{p}\right)_{0}
\end{aligned}
$$

Another convenient way to write this is in a $3 \times 3$ matrix form

$$
\left(\begin{array}{c}
x \\
x^{\prime} \\
\frac{\Delta p}{p}
\end{array}\right)=\left(\begin{array}{ccc}
\cos \frac{\sqrt{1-n} s}{\rho} & \frac{\rho}{\sqrt{1-n}} \sin \frac{\sqrt{1-n} s}{\rho} & \frac{\rho}{1-n}\left(1-\cos \frac{\sqrt{1-n} s}{\rho}\right) \\
-\frac{\sqrt{1-n}}{\rho} \sin \frac{\sqrt{1-n} s}{\rho} & \cos \frac{\sqrt{1-n} s}{\rho} & \frac{1}{\sqrt{1-n}} \sin \frac{\sqrt{1-n} s}{\rho} \\
0 & 0 & 1
\end{array}\right)\left(\begin{array}{c}
x_{0} \\
x_{0}^{\prime} \\
\left(\frac{\Delta p}{p}\right)_{0}
\end{array}\right)
$$

\section{Transfer matrices and momentum dispersion:}

As we deal with $\beta(s)$, we have a similar way to determine the momentum dispersion function $D(s)$ for any position by choosing the starting point of the cell. Here, the transfer matrix for any part is

$$
M_{p} n(s)=\left(\begin{array}{ccc}
\cos \frac{\sqrt{1-n} s}{\rho} & \frac{\rho}{\sqrt{1-n}} \sin \frac{\sqrt{1-n} s}{\rho} & \frac{\rho}{1-n}\left(1-\cos \frac{\sqrt{1-n} s}{\rho}\right) \\
-\frac{\sqrt{1-n}}{\rho} \sin \frac{\sqrt{1-n} s}{\rho} & \cos \frac{\sqrt{1-n} s}{\rho} & \frac{1}{\sqrt{1-n}} \sin \frac{\sqrt{1-n} s}{\rho} \\
0 & 0 & 1
\end{array}\right)
$$

For a cell, the transfer matrix can be written as

$$
M_{p}=\left(\begin{array}{ccc}
a & b & D(s) \\
c & d & D^{\prime}(s) \\
0 & 0 & 1
\end{array}\right)
$$

where $a, b, c$ and $d$ are the same as before. 
$D(s)$ should have a maximal value in the middle of the intervals and a minimal value in the middle of quads. Therefore,

$$
\begin{aligned}
& D_{\text {max }} / R=M_{p i}[1,3] / R=\left(M_{p 0}\left(s=l_{i} / 2\right) \cdot M_{p n}\left(s=l_{q}\right) \cdot M_{p 0}\left(s=l_{i} / 2\right)\right)[1,3] / R \\
& D_{\text {min }} / R=M_{p q}[1,3] / R=\left(M_{p n}\left(s=l_{q} / 2\right) \cdot M_{p 0}\left(s=l_{i}\right) \cdot M_{p n}\left(s=l_{q} / 2\right)\right)[1,3] / R
\end{aligned}
$$

with $M_{p 0}$ is $M_{p n}$ for $n=0$.

Here are our results:

$$
\begin{aligned}
\frac{D_{\min }}{R}= & \frac{1-\cos \left(\pi \nu_{x} / 2\right)}{1-n_{0}}-\frac{2 n_{0}}{1-n_{0}} \sin ^{2}\left(\frac{l_{i}}{2 R}\right) \cos \left(\frac{\sqrt{1-n_{0}} l_{q}}{2 R}\right) \\
& -\frac{n_{0} \sqrt{1-n_{0}}}{\left(1-n_{0}\right)^{2}} \sin \left(\frac{l_{i}}{R}\right) \sin \left(\frac{\sqrt{1-n_{0}} l_{q}}{2 R}\right) \\
\frac{D_{\max }}{R}= & 1-\cos \left(\pi \nu_{x} / 2\right)+\frac{2 n_{0}}{1-n_{0}} \cos \left(\frac{l_{i}}{2 R}\right) \sin ^{2}\left(\frac{\sqrt{1-n_{0}} l_{q}}{2 R}\right) \\
& +\frac{n_{0}}{\sqrt{1-n_{0}}} \sin \left(\frac{l_{i}}{2 R}\right) \sin \left(\frac{\sqrt{1-n_{0}} l_{q}}{R}\right)
\end{aligned}
$$


APPENDIX C: GEOMETRY FACTOR FOR FAST ROTATION ANALYSIS 


\section{C.1 Geometry Factor of Square Pulse}

Based on the time cut positions, we can divide the skewness into several cases. The geometry factor is then calculated by using the cut area.

Case A: $0 \leq \delta<\Delta / 2$

1. case A1: $\delta+\Delta / 2 \leq x$ (Fig. C.1a): Here, $\beta(i, j)=0$.

2. case A2: $\Delta / 2 \leq x<\delta+\Delta / 2$ (Fig. C.1b): Here, we have $\frac{a}{b / 2}=\frac{\delta+\Delta / 2-x}{\delta}$.

$$
\beta(i, j)=\frac{S_{1}}{S_{2}}=\frac{1}{2}\left(\frac{a}{b / 2}\right)^{2}=\frac{1}{2}\left(1+\frac{\Delta / 2-x}{\delta}\right)^{2}=\frac{1}{2}-\frac{x}{\delta}+\frac{\Delta}{2 \delta}+\frac{1}{2 \delta^{2}}(x-\Delta / 2)^{2}
$$

3. case A3: $\Delta / 2-\delta \leq x<\Delta / 2$ (Fig. C.1c): Here, we have $\frac{b-a}{b / 2}=\frac{x-(\Delta / 2-\delta)}{\delta}$.

$$
\beta(i, j)=\frac{S_{1}}{S_{2}}=\frac{S_{2}-S_{3}}{S_{2}}=1-\frac{1}{2}\left(\frac{b-a}{b / 2}\right)^{2}=\frac{1}{2}-\frac{x}{\delta}+\frac{\Delta}{2 \delta}-\frac{1}{2 \delta^{2}}(x-\Delta / 2)^{2}
$$

4. case A5: $0 \leq x<\Delta / 2-\delta$ (Fig. C.1d): Here, $\beta(i, j)=\frac{S_{1}}{S_{2}}=1$.

Case B: $\Delta / 2 \leq \delta<\Delta$

1. case B1: $\delta+\Delta / 2 \leq x$ (Fig. C.2a): Here, $\beta(i, j)=0$.

2. case B2: $\Delta / 2 \leq x<\delta+\Delta / 2$ (Fig. C.2b) : Here, we have $\frac{a}{b / 2}=\frac{\delta+\Delta / 2-x}{\delta}$.

$$
\beta(i, j)=\frac{S_{1}}{S_{2}}=\frac{1}{2}\left(\frac{a}{b / 2}\right)^{2}=\frac{1}{2}\left(1+\frac{\Delta / 2-x}{\delta}\right)^{2}=\frac{1}{2}-\frac{x}{\delta}+\frac{\Delta}{2 \delta}+\frac{1}{2 \delta^{2}}(x-\Delta / 2)^{2}
$$

3. case B3: $\Delta / 2-\delta \leq x<\Delta / 2$ (Fig. C.2c): Here, we have $\frac{b-a}{b / 2}=\frac{x-(\Delta / 2-\delta)}{\delta}$.

$$
\beta(i, j)=\frac{S_{1}}{S_{2}}=\frac{S_{2}-S_{3}}{S_{2}}=1-\frac{1}{2}\left(\frac{b-a}{b / 2}\right)^{2}=\frac{1}{2}-\frac{x}{\delta}+\frac{\Delta}{2 \delta}-\frac{1}{2 \delta^{2}}(x-\Delta / 2)^{2}
$$




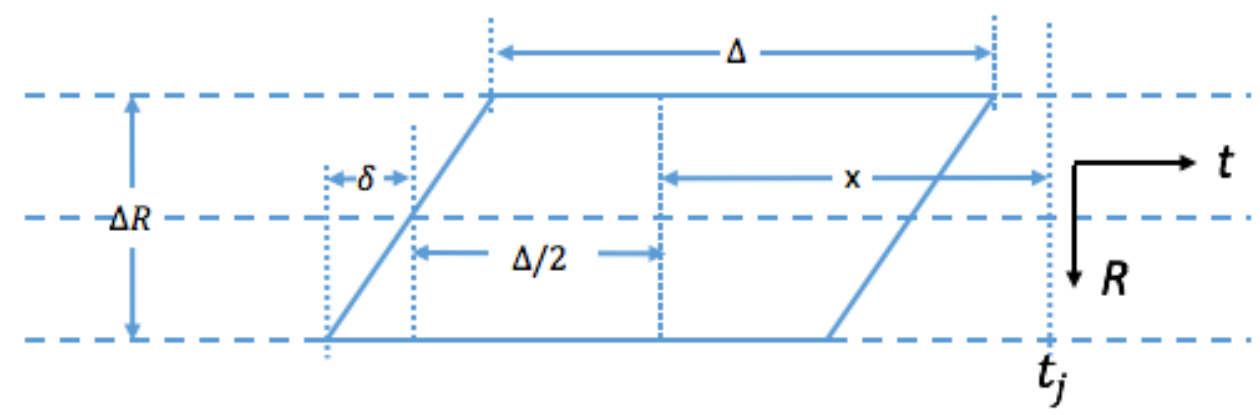

(a) Square pulse case A1.

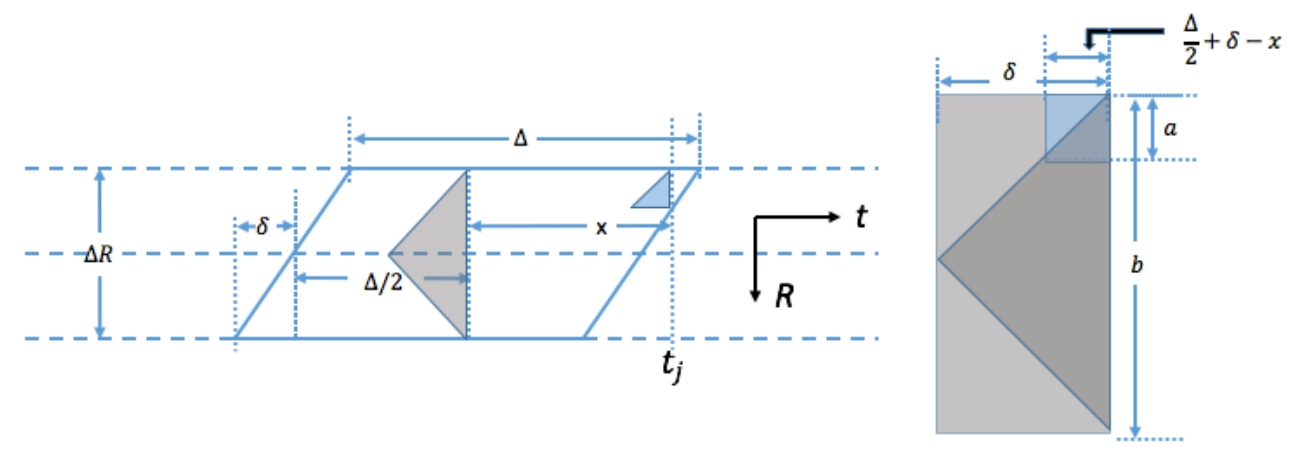

(b) Square pulse case A2.
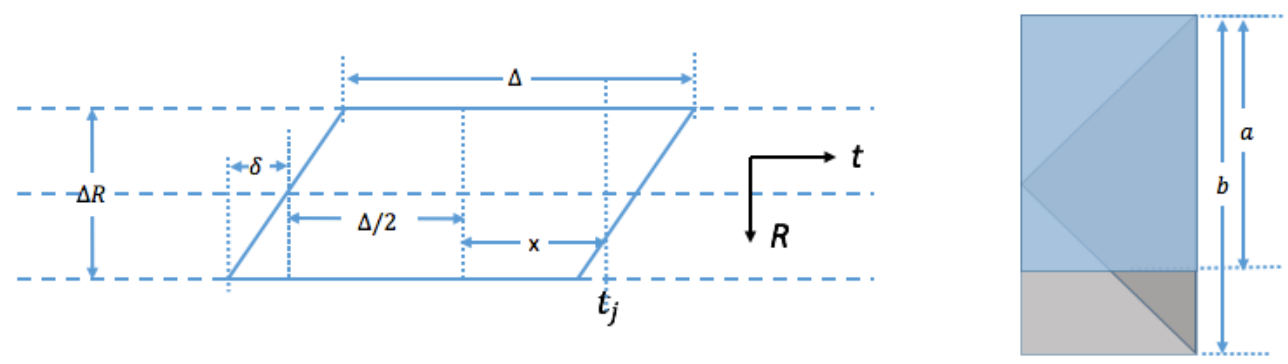

(c) Square pulse case A3.
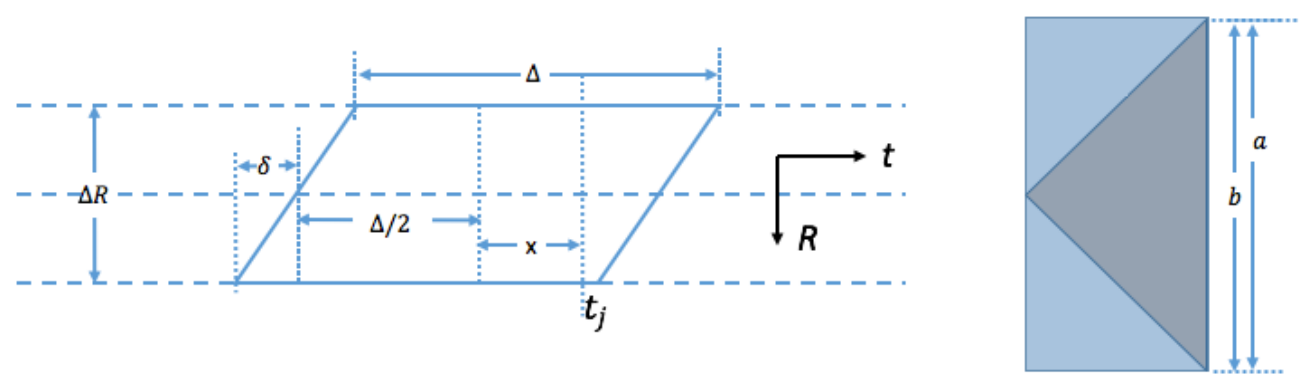

(d) Square pulse case A5.

Figure C.1: Square pulse case A. 
4. case B6: $0 \leq x<\delta-\Delta / 2$ (Fig. C.2d): Here, we have $\frac{a}{b / 2}=\frac{\delta-\Delta / 2-x}{\delta}$ and $\frac{c}{b / 2}=\frac{\delta-\Delta / 2+x}{\delta}$.

$$
\beta(i, j)=\frac{S_{1}}{S_{2}}=1-\frac{S_{3}}{S_{2}}-\frac{S_{4}}{S_{2}}=1-\frac{1}{2}\left(\frac{a}{b / 2}\right)^{2}-\frac{1}{2}\left(\frac{c}{b / 2}\right)^{2}=\frac{\Delta}{\delta}-\left(\frac{x}{\delta}\right)^{2}-\left(\frac{\Delta}{2 \delta}\right)^{2}
$$

Case C: $\Delta<\delta$

1. case C1: $\delta+\Delta / 2 \leq x$ (Fig. C.3a): Here, $\beta(i, j)=0$.

2. case $\mathrm{C} 2: \delta-\Delta / 2 \leq x<\delta+\Delta / 2$ (Fig. C.3b): Here, we have $\frac{a}{b / 2}=\frac{\delta+\Delta / 2-x}{\delta}$.

$$
\beta(i, j)=\frac{S_{1}}{S_{2}}=\frac{1}{2}\left(\frac{a}{b / 2}\right)^{2}=\frac{1}{2}\left(1+\frac{\Delta / 2-x}{\delta}\right)^{2}=\frac{1}{2}-\frac{x}{\delta}+\frac{\Delta}{2 \delta}+\frac{1}{2 \delta^{2}}(x-\Delta / 2)^{2}
$$

3. case C4: $\Delta / 2 \leq x<\delta-\Delta / 2$ (Fig. C.3c): Here, we have $\frac{a}{b / 2}=\frac{\delta-\Delta / 2-x}{\delta}$ and $\frac{c}{b / 2}=$ $\frac{\delta+\Delta / 2-x}{\delta}$.

$$
\beta(i, j)=\frac{S_{1}}{S_{2}}=\frac{S_{3}-S_{4}}{S_{2}}=\frac{S_{3}}{S_{2}}-\frac{S_{4}}{S_{2}}=\frac{1}{2}\left(\frac{c}{b / 2}\right)^{2}-\frac{1}{2}\left(\frac{a}{b / 2}\right)^{2}=\frac{\Delta}{\delta}-\frac{x \Delta}{\delta^{2}}
$$

4. case C6: $0 \leq x<\Delta / 2$ (Fig. C.3d): Here, we have $\frac{a}{b / 2}=\frac{\delta-\Delta / 2-x}{\delta}$ and $\frac{c}{b / 2}=\frac{\delta-\Delta / 2+x}{\delta}$.

$$
\beta(i, j)=\frac{S_{1}}{S_{2}}=1-\frac{S_{3}}{S_{2}}-\frac{S_{4}}{S_{2}}=1-\frac{1}{2}\left(\frac{a}{b / 2}\right)^{2}-\frac{1}{2}\left(\frac{c}{b / 2}\right)^{2}=\frac{\Delta}{\delta}-\left(\frac{x}{\delta}\right)^{2}-\left(\frac{\Delta}{2 \delta}\right)^{2}
$$

\section{C.2 Geometry Factor of Graziano Pulse}

Following the same procedure as for square pulse, we also divide the skewness of a Graziano pulse into several cases.

\section{Case A:}

1. case A1: $\delta+\Delta / 2 \leq x$ (Fig. C.4a): Here, $\beta(i, j)=0$. 


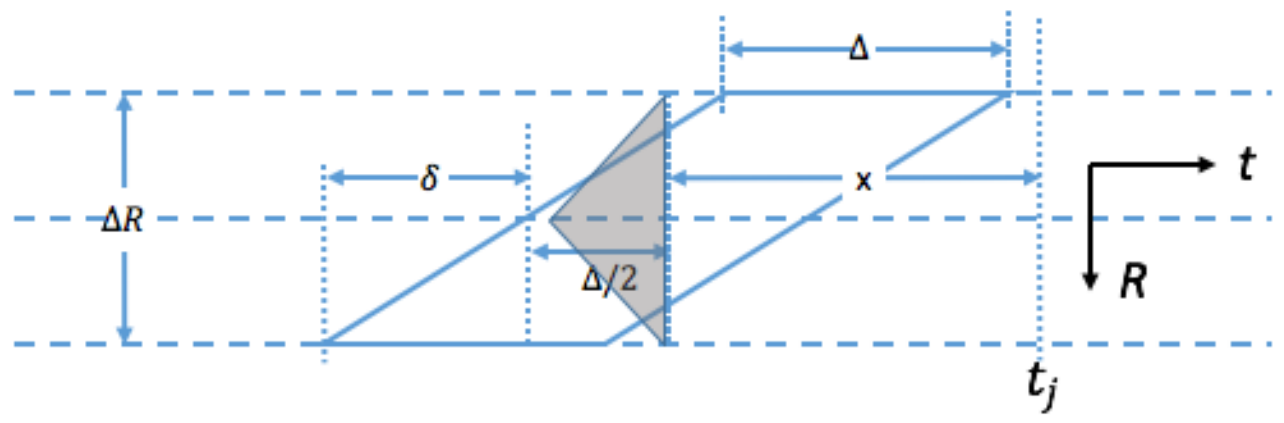

(a) Square pulse case B1.

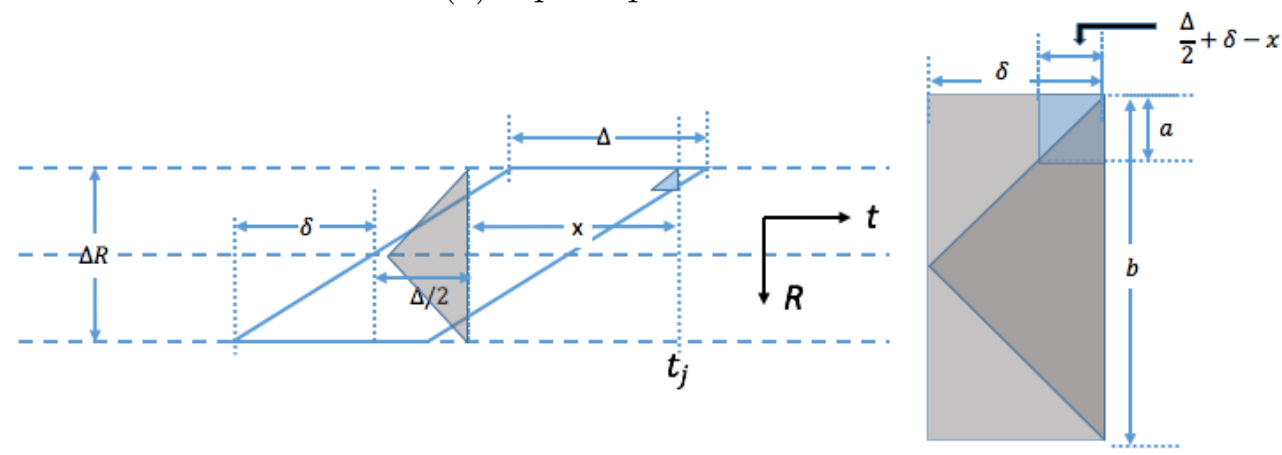

(b) Square pulse case B2.
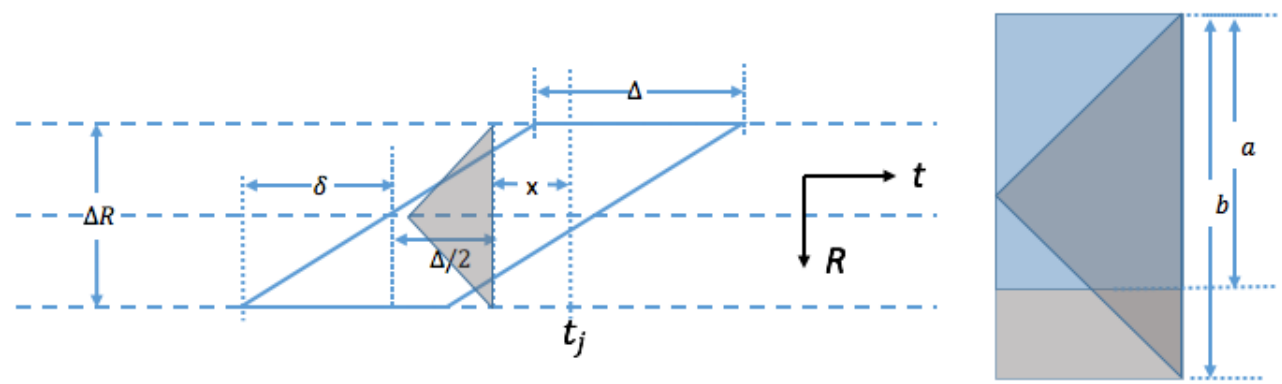

(c) Square pulse case B3.
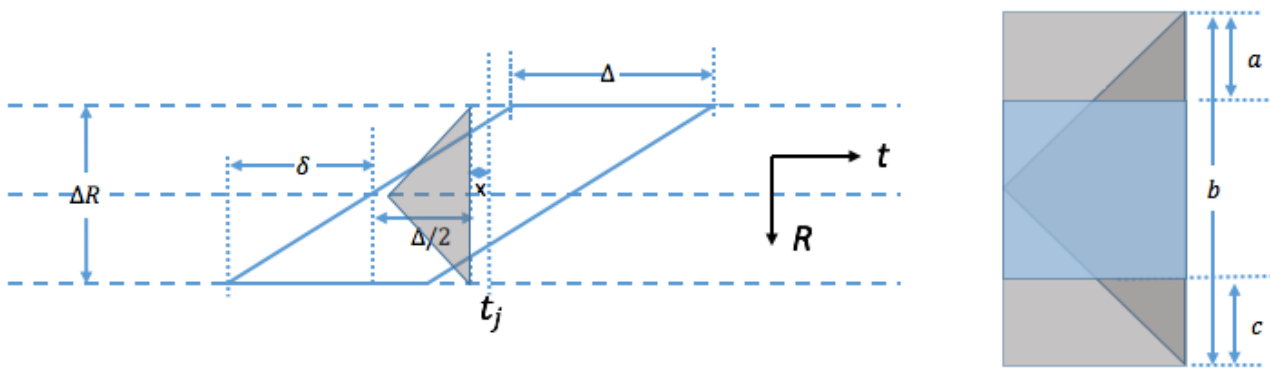

(d) Square pulse case B6.

Figure C.2: Square pulse case B. 


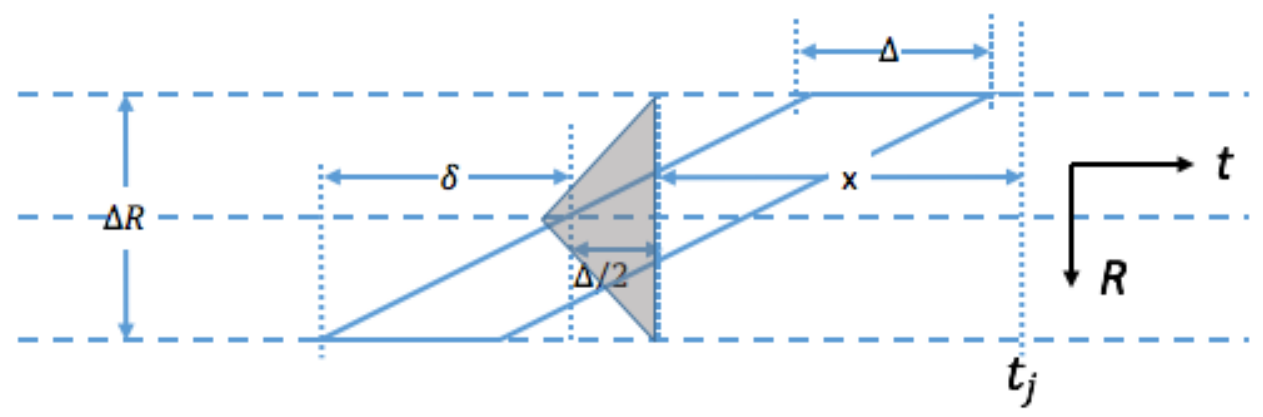

(a) Square pulse case C1.
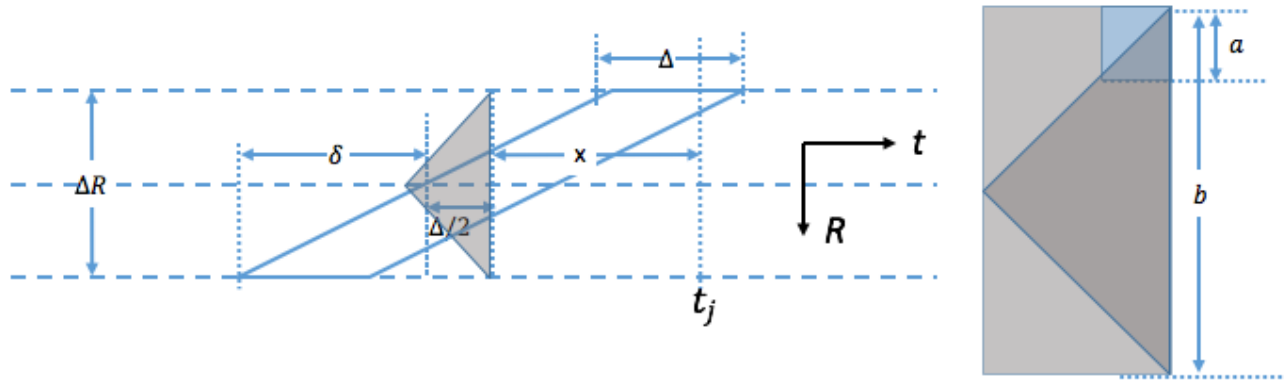

(b) Square pulse case C2.

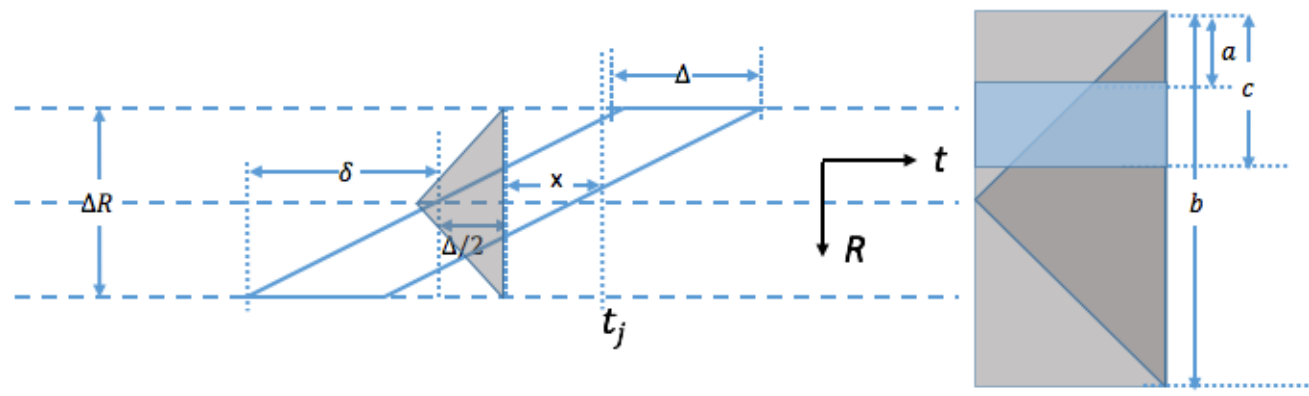

(c) Square pulse case C4.
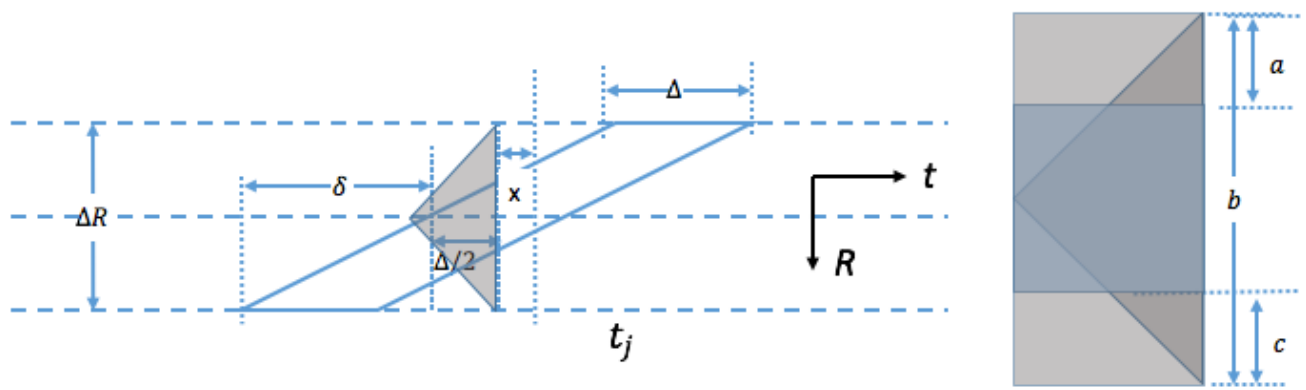

(d) Square pulse case C6.

Figure C.3: Square pulse case C. 


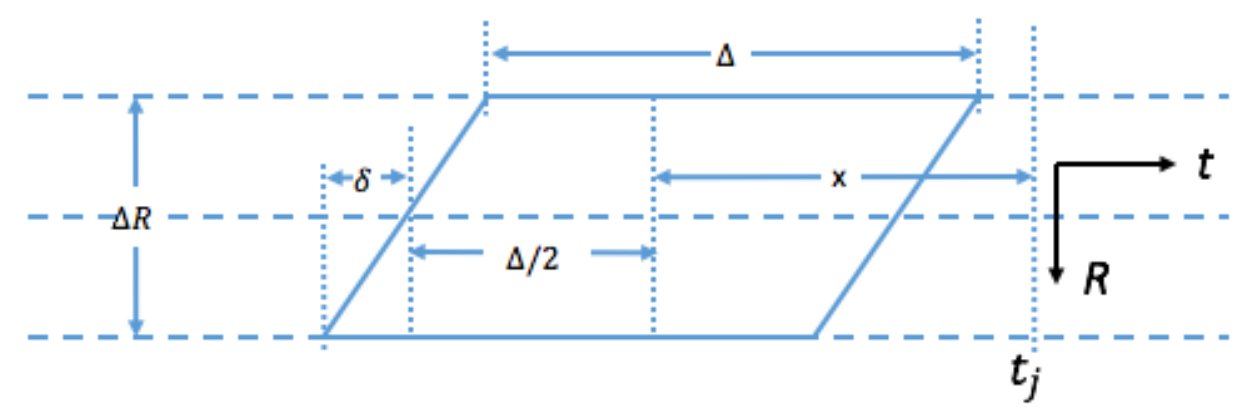

(a) Graziano pulse case A1.
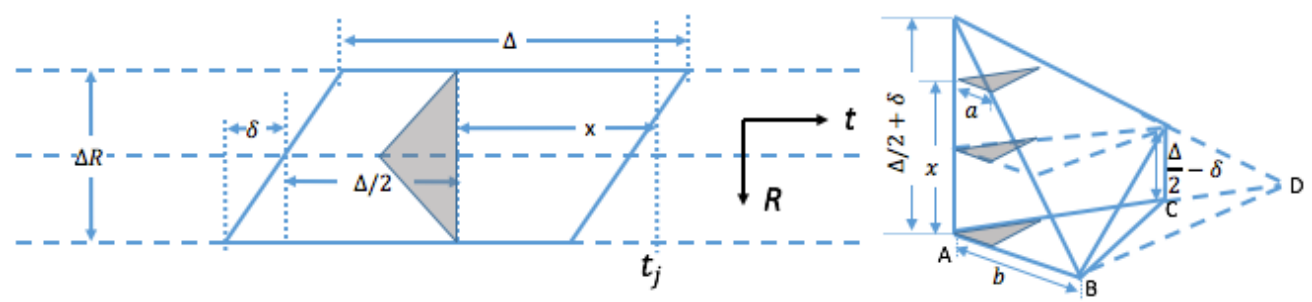

(b) Graziano pulse case A3.
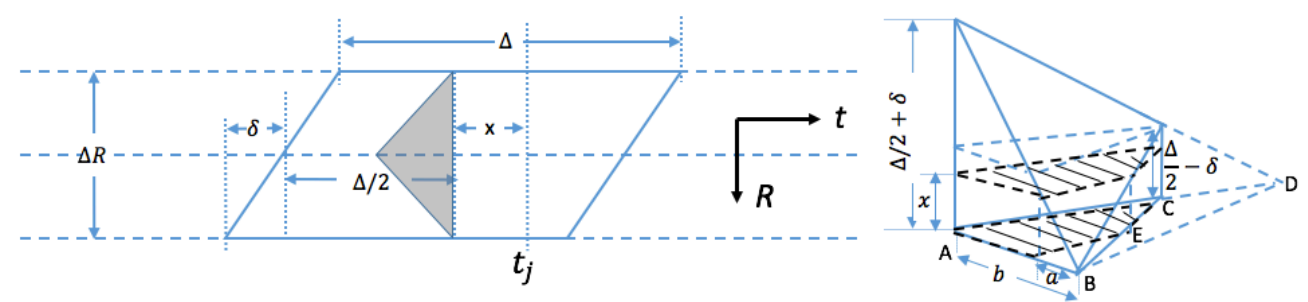

(c) Graziano pulse case A5.

Figure C.4: Graziano pulse case A.

2. case A3: $\Delta / 2-\delta \leq x<\delta+\Delta / 2$ (Fig. C.4b): Here, we have $\frac{a}{b}=\frac{\Delta / 2+\delta-x}{\Delta / 2+\delta}$ and $\frac{A C}{A D}=\frac{2 \delta}{\Delta / 2+\delta}$.

$$
\beta(i, j)=\frac{S_{1}}{S_{2}}=\frac{S_{1}}{\frac{A C}{A D} S_{3}}=\frac{A D}{A C}\left(\frac{a}{b}\right)^{2}=\frac{\left(\frac{\Delta}{2}+\delta-x\right)^{2}}{2 \delta\left(\frac{\Delta}{2}+\delta\right)}
$$

3. case A5: $0 \leq x<\Delta / 2-\delta$ (Fig. C.4c): Here, we have $\frac{a}{b}=\frac{x}{\Delta / 2+\delta}$ and $\frac{B E}{B C}=\frac{x}{\Delta / 2-\delta}$.

$$
\beta(i, j)=\frac{S_{1}}{S_{2}}=\frac{S_{2}-S_{3}}{S_{2}}=1-\frac{a}{b} \frac{B E}{B C}=1-\frac{x^{2}}{\left(\frac{\Delta}{2}\right)^{2}-\delta^{2}}
$$

\section{Case B:}


1. case B1: $\delta+\Delta / 2 \leq x$ (Fig. C.5a): Here, $\beta(i, j)=0$.

2. case B3: $\delta-\Delta / 2 \leq x<\delta+\Delta / 2$ (Fig. C.5b): Here, we have $\frac{a}{b}=\frac{\frac{\Delta}{2}+\delta-x}{\frac{\Delta}{2}+\delta}$ and $\frac{c}{d}=\frac{\frac{\Delta}{2}+\delta-x}{2 \delta}$.

$$
\beta(i, j)=\frac{S_{1}}{S_{2}}=\frac{a}{b} \frac{c}{d}=\frac{\left.\frac{(\Delta}{2}+\delta-x\right)^{2}}{2 \delta\left(\frac{\Delta}{2}+\delta\right)}
$$

3. case B6: $0 \leq x<\delta-\Delta / 2$ (Fig. C.5c): Here, we have $D F=E G, I F\|C G, D H\| C E$ and $E G / A B=\Delta / 2 \delta$.

$$
\begin{array}{r}
\frac{I J}{C G}=1-\frac{F I}{C G}=1-\frac{\frac{\Delta}{2}+\delta-x}{\frac{\Delta}{2}+\delta}=\frac{x}{\frac{\Delta}{2}+\delta} \\
\frac{H J}{C D}=1-\frac{D H}{C E}=1-\frac{\delta-\frac{\Delta}{2}-x}{\delta-\frac{\Delta}{2}}=\frac{x}{\delta-\frac{\Delta}{2}} \\
\beta(i, j)=\frac{S_{1}}{S_{2}}=\frac{E G}{A B}\left(1-\frac{I J}{C G} \frac{H J}{C D}\right)=\frac{\Delta}{2 \delta}\left[1-\frac{x^{2}}{\delta^{2}-\left(\frac{\Delta}{2}\right)^{2}}\right]
\end{array}
$$




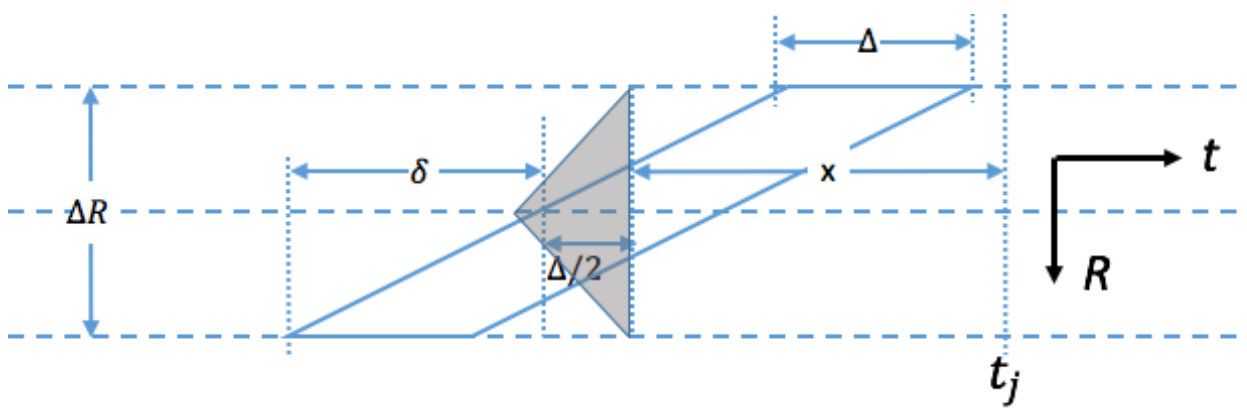

(a) Graziano pulse case B1.

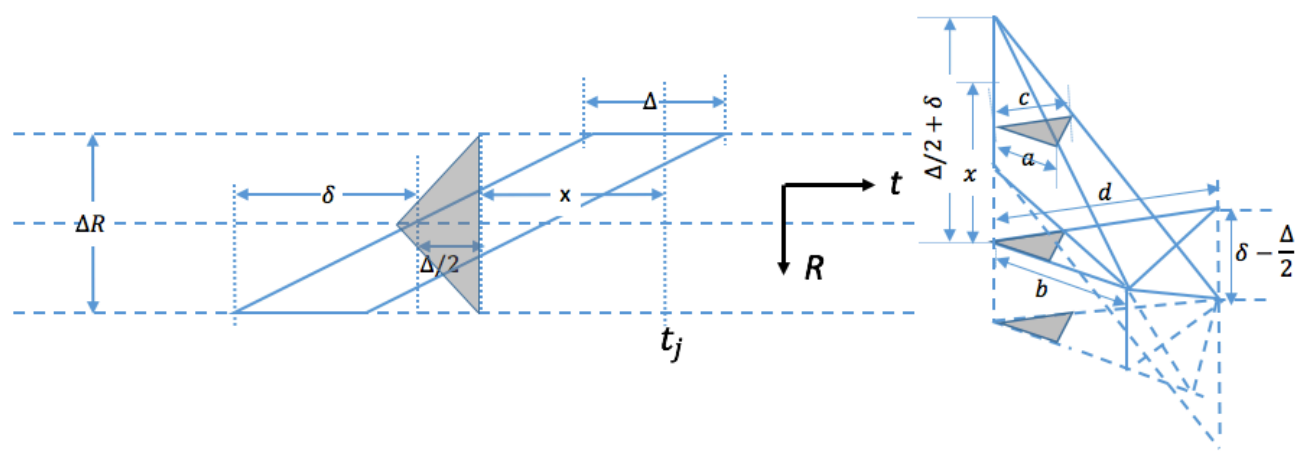

(b) Graziano pulse case B3.
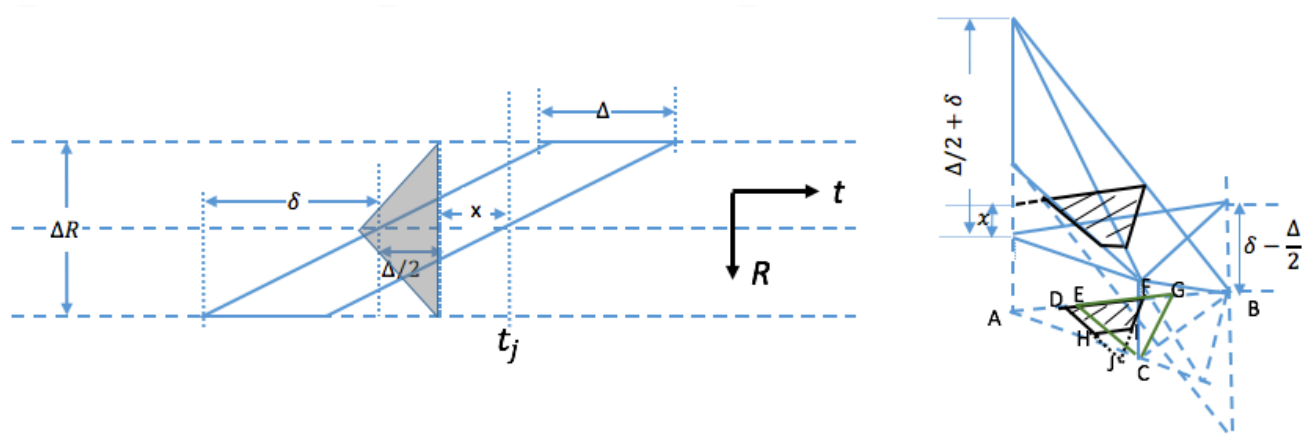

(c) Graziano pulse case B6.

Figure C.5: Graziano pulse case B. 


\section{VITA}

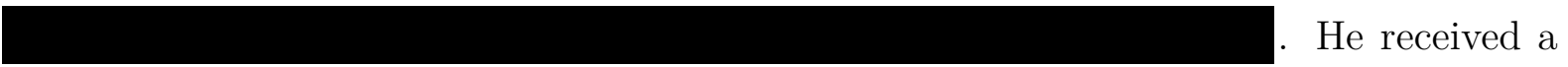

B.S. in physics from Sichuan University in Chengdu, Sichuan Province China in 2010.

Wanwei began his graduate program at the University of Mississippi in the summer of 2013. He received a M.S. in physics there in May 2015 with the thesis "Semi-leptonic Decay of $\lambda_{b}$ in the Standard Model and with New Physics", supervised by Dr. Alakabha Datta. After that, he moved to Fermilab to perform the work described in this dissertation on the Fermilab Muon g-2 Experiment supervised by Dr. Breese Quinn. He expects to receive a Ph.D. from the University of Mississippi in May 2018.

Following the completion of his Ph.D., Wanwei will take a position as a Postdoctoral Research Associate in the Neutrino Division at Fermilab. 